\title{
Atroposelective Construction of Arylindoles by Chiral Phosphoric Acid-Catalyzed Cross-Coupling of Indoles and Quinones
}

\author{
Dong-Liang Lu, ${ }^{\dagger}$ Ye-Hui Chen, ${ }^{\ddagger}$ Shao-Hua Xiang, ${ }^{\S}$ Peiyuan $\mathrm{Yu},{ }^{\ddagger}$ Bin Tan,${ }^{\ddagger}$ and Shaoyu $\mathrm{Li}^{*}, \S$
}

†Key Laboratory of Jiangxi University for Functional Materials Chemistry, Gannan Normal University; Ganzhou 341000, China

Department of Chemistry and Shenzhen Grubbs Institute, Southern University of Science and Technology, Shenzhen 518055, China

${ }^{\S}$ Academy for Advanced Interdisciplinary Studies and Department of Chemistry, Southern University of Science and Technology, Shenzhen, 518055, China.

\section{Table of concents}

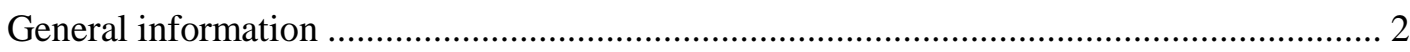

Synthetic procedures and characterization of substrates …............................................... 3

General procedure for preparation of racemic samples .................................................... 6

Reaction optimization of 3a-3p and their synthetic procedures ....................................... 7

Reaction optimization of $3 \mathrm{q}-3 \mathrm{u}$ and their synthetic procedures ........................................ 10

Reaction optimization of 4a-4g and their synthetic procedures ....................................... 13

Experimental measurement of enantiomerization barrier for $3 \mathrm{a}$ and $4 \mathrm{a}$............................. 43

X-ray crystal structural parameters of absolute configuration of $3 \mathrm{e}$ and $3 \mathrm{q} \ldots \ldots \ldots \ldots \ldots \ldots \ldots \ldots . . . . . . . . . .45$

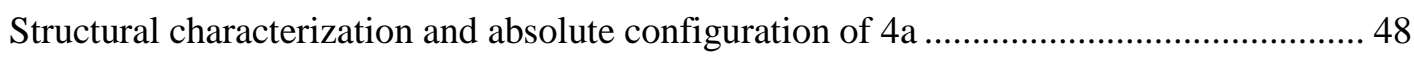

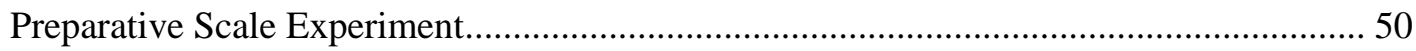

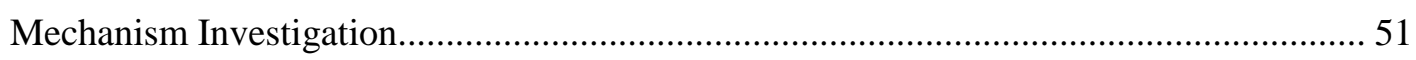

Application of 4a in addition of diethylzinc to aldehyde …….......................................... 55

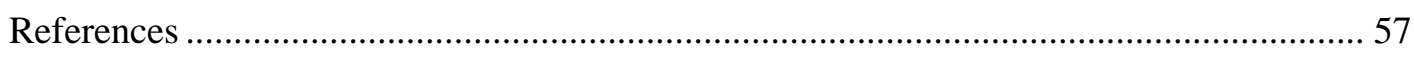

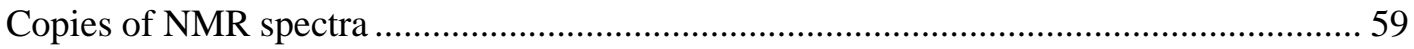




\section{General information}

Analytical thin layer chromatography (TLC) was performed on silica gel 60 GF254 plates. Flash column chromatography was performed using silicycle silica gel (pore size 60A). Visualization on TLC was realized by use of UV light $(254 \mathrm{~nm}) .{ }^{1} \mathrm{H}$ and ${ }^{13} \mathrm{C}$ NMR spectra were measured on Bruker DPX 400 spectrometer using $\mathrm{CD}_{2} \mathrm{Cl}_{2}, \mathrm{CDCl}_{3}$ or acetone- $\mathrm{d}_{6}$ with tetramethylsilane (TMS) as the internal reference. The data for ${ }^{1} \mathrm{H}$ NMR are recorded in $\mathrm{ppm}$ as $(\mathrm{s}=$ singlet, $\mathrm{d}=$ doublet, $\mathrm{t}=$ triplet, $\mathrm{q}=$ quadruplet, $\mathrm{m}=$ multiplet, brs $=$ broad singlet, $\mathrm{brm}=$ broad multiplet, coupling constant(s) in $\mathrm{Hz}$, integration). Data for ${ }^{13} \mathrm{C}$ NMR are reported in terms of chemical shift $(\delta, \mathrm{ppm})$. The enantiomeric excess values were recorded on Agilent 1200 LC instrument and a Daicel CHIRALCEL IA/IB/IG/AD-H/OD-H/OD3 column. High resolution mass spectroscopy (HRMS) analyses were performed at a Bruker Daltonics. Inc mass instrument (ESI). 


\section{Synthetic procedures and characterization of substrates}

(a) Substrates involved in the manuscript

Quinone derivatives<smiles>CCOC(=O)C1=CC(=O)C=CC1=O</smiles><smiles>CCCOC(=O)C1=CC(=O)C=CC1=O</smiles><smiles>CCCCOC(=O)C1=CC(=O)C=CC1=O</smiles><smiles>O=C1C=CC(=O)C(C(=O)OCc2ccccc2)=C1</smiles><smiles>O=C1C=C(I)C(=O)C=C1I</smiles><smiles>[R]OC(=O)C1CC(=O)C=CC1=O</smiles>

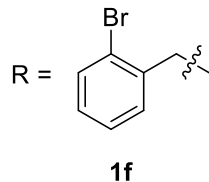<smiles>CC(C)(C)c1cccc(Br)c1</smiles><smiles>CC(C)(C)c1ccc(Br)cc1</smiles><smiles>CCc1ccc2ccccc2c1</smiles><smiles>[R17]=C1C=C(Cl)C(=O)C=C1Cl</smiles><smiles>[R1]c1ccc(S(C)(=O)=O)cc1</smiles><smiles>CS(=O)(=O)c1ccc([N+](=O)[O-])cc1</smiles><smiles>O=S(=O)(O)c1ccc2ccccc2c1</smiles>

Substituted indoles<smiles>CC(C)c1cc2ccccc2[nH]1</smiles>

2a<smiles>CCCC(C)(C)c1cc2ccccc2[nH]1</smiles><smiles>COc1ccc2[nH]c(C)cc2c1</smiles>

2k<smiles>Cc1ccc2cc(C(C)(C)C)[nH]c2c1</smiles>

2b<smiles>COc1ccc2[nH]c(C(C)(C)C)cc2c1</smiles>

2c<smiles>CC(C)(C)c1cc2cc(Cl)ccc2[nH]1</smiles>

2d<smiles>CC(C)c1cc2cc(Br)ccc2[nH]1</smiles><smiles>Cc1ccc2[nH]c(C)cc2c1</smiles>

HO<smiles>Cc1cc2cc(Br)ccc2[nH]1</smiles><smiles>CCOC(=O)c1cc2cc(O)ccc2[nH]1</smiles><smiles>COc1ccc2[nH]ccc2c1</smiles>
20<smiles>NC(=O)c1cc2cc(O)ccc2[nH]1</smiles>

$2 p$<smiles>CC(=O)c1cc2cc(O)ccc2[nH]1</smiles>

$2 q$<smiles>Cc1cc2cc(O)ccc2n1C(=O)OC(C)(C)C</smiles><smiles>Cn1c(C(C)(C)C)cc2ccccc21</smiles>

\section{(b) Synthesis of new compounds}

Quinone derivatives 1 were synthesized according to the literature procedures. ${ }^{1,2}$

Substituted indoles $\mathbf{2}$ were also prepared according to the literature procedures. ${ }^{3,4}$ 


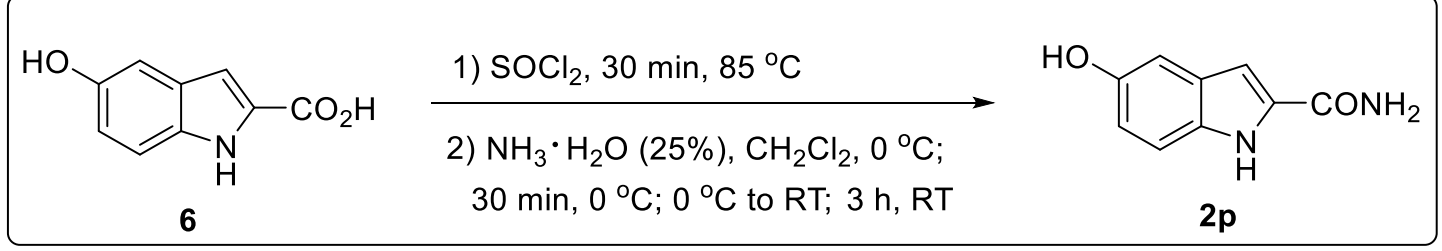

A mixture of 5-Hydroxyindole-2-carboxylic acid $6(500 \mathrm{mg}, 2.82 \mathrm{mmol})$ in $\mathrm{SOCl}_{2}(8 \mathrm{~mL})$ was refluxed at $85{ }^{\circ} \mathrm{C}$. 30 minutes later. The reaction was stopped and cooled to RT. The reaction solution was concentrated to give an acyl chloride which was used for next reaction without purification.

To aqueous ammonia solution $(25 \%, 16 \mathrm{~mL})$ was injected a solution of acyl chloride prepared above in anhydrous dichloromethane $(8 \mathrm{~mL})$ dropwise in an ice bath. 30 minutes later, the reaction solution was warmed to room temperature and stirred for another 3 hours. Then the reaction solution was diluted with ethyl acetate $(20 \mathrm{~mL})$, separated. The aqueous layer was extracted with ethyl acetate $(15 \mathrm{~mL} \times 2)$ and the combined organic layer was washed with brine $(10 \mathrm{~mL} \times 2)$, dried with anhydrous $\mathrm{Na}_{2} \mathrm{SO}_{4}$ and finally concentrated in vacuo. This residue was purified by recrystallization (ethyl acetate/hexane) to give 5-Hydroxyindole-2carboxamide pale yellow solid (380 mg, 77\%).

${ }^{1}$ H NMR (400 MHz, Acetone-d 6 ) $\delta 10.69$ (s, 1H), 7.87 (s, 1H), 7.49 (s, 1H), 7.39 (d, $J=12.8$ $\mathrm{Hz}, 1 \mathrm{H}), 7.00(\mathrm{~d}, J=2.2 \mathrm{~Hz}, 2 \mathrm{H}), 6.86(\mathrm{~m}, 2 \mathrm{H})$.

${ }^{13}$ C NMR (100 MHz, Acetone-d $) \delta=164.24,152.35,132.85,132.59,129.50,115.71$, 113.66, 105.59, 103.23.<smiles>CC(=O)c1cc2cc(O)ccc2[nH]1</smiles>

According to the literature method. ${ }^{5}$

${ }^{1}$ H NMR (400 MHz, Acetone-d $\left.{ }_{6}\right) \delta 10.71(\mathrm{~s}, 1 \mathrm{H}), 7.99$ (s, 1H), 7.39 (d, $\left.J=8.8 \mathrm{~Hz}, 1 \mathrm{H}\right), 7.04$ (dd, $J=13.8,1.6 \mathrm{~Hz}, 2 \mathrm{H}), 6.93$ (dd, $J=8.8,2.3 \mathrm{~Hz}, 1 \mathrm{H}), 3.86(\mathrm{~s}, 3 \mathrm{H})$.

${ }^{13}$ C NMR (100 MHz, Acetone-d $\left.{ }_{6}\right) \delta=162.71,152.57,133.50,129.02,128.59,117.06$, $113.86,107.89,105.70,51.89$. 


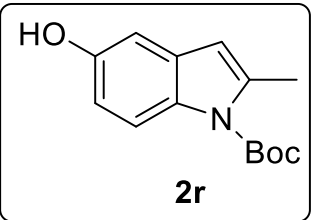

According to the literature method. ${ }^{6}$

${ }^{1}$ H NMR (400 MHz, CDCl $) \delta 7.95(\mathrm{~d}, J=8.9 \mathrm{~Hz}, 1 \mathrm{H}), 6.87(\mathrm{~d}, J=2.5 \mathrm{~Hz}, 1 \mathrm{H}), 6.77(\mathrm{dd}, J$ $=8.9,2.5 \mathrm{~Hz}, 1 \mathrm{H}), 6.19(\mathrm{~s}, 1 \mathrm{H}), 5.70(\mathrm{~s}, 1 \mathrm{H}), 2.57(\mathrm{~s}, 3 \mathrm{H}), 1.67(\mathrm{~s}, 9 \mathrm{H})$.

${ }^{13}$ C NMR (100 MHz, $\left.\mathbf{C D C l}_{3}\right) \delta=151.59,150.80,138.84,131.35,130.57,116.33,111.78$, $107.84,104.94,83.71,28.37,17.27$. 


\section{General procedure for preparation of racemic samples}

(a) Synthesis of racemic samples 3

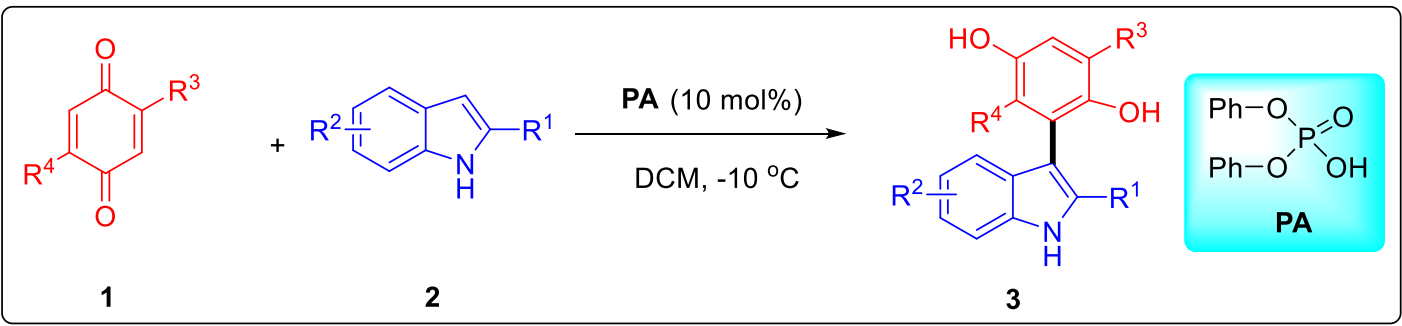

Quinone $1(0.10 \mathrm{mmol})$ was added to a solution of indole $2(0.12 \mathrm{mmol})$ and phosphoric acid $(10 \mathrm{~mol} \%)$ in DCM $(2.0 \mathrm{~mL})$, and the resulting mixture was stirred at $-10{ }^{\circ} \mathrm{C}$ until the transformation was completed (detected by TLC). Solvent was removed under reduced pressure and the crude residue was purified by column chromatography on silica-gel to give pure racemic product 3 .

\section{(b) Synthesis of racemic samples 4}

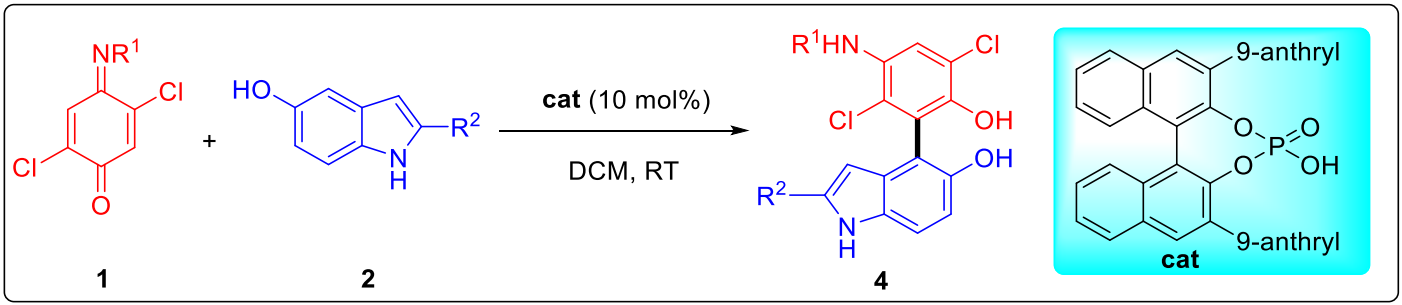

To a stirred solution of cat (10 mol\%) and substituted indole $2(0.12 \mathrm{mmol})$ in DCM (1.0 mL) was added iminoquinone derivative $1(0.1 \mathrm{mmol})$, and the reaction mixture was stirred at room temperature for $2 \mathrm{~h}$. After removing the solvent, the crude residue was purified by column chromatography on silica-gel to give pure racemic product 4 . 


\section{Reaction optimization of $3 a-3 p$ and their synthetic procedures}

\section{(a) Synthesis of chiral phosphoric acids}

Chiral phosphoric acids (CPA 1-8) were prepared according to the literature method with slight change in post-treatment. ${ }^{7}$

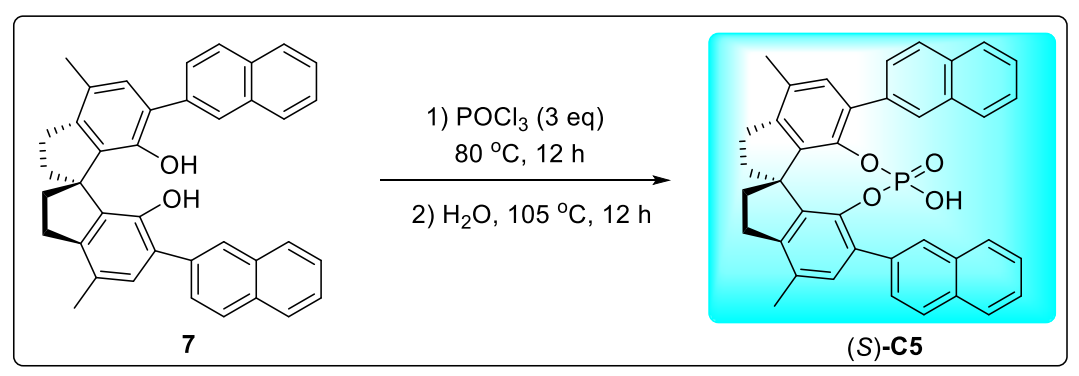

Under argon atmosphere, $\mathrm{POCl}_{3}(9.0 \mathrm{mmol})$ was added to the solution of $7(3.0 \mathrm{mmol})$ in pyridine $(15 \mathrm{~mL})$ at $0{ }^{\circ} \mathrm{C}$, the mixture next was heated to $80{ }^{\circ} \mathrm{C}$ for $12 \mathrm{~h}$. Cooling the reaction to $0{ }^{\circ} \mathrm{C}$, following by droping $15 \mathrm{~mL}$ of water slowly, the yielding mixture was stirred again at $105{ }^{\circ} \mathrm{C}$ for another $12 \mathrm{~h}$. After being chilled to $0{ }^{\circ} \mathrm{C}$, the reaction was diluted with DCM (150 $\mathrm{mL})$, washed with $3 \mathrm{~N} \mathrm{HCl}(3 \times 30 \mathrm{~mL})$, and saturated brine $(3 \times 30 \mathrm{~mL})$. Combing the organic layer and removing solvent, then the crude product was purified by silica gel column chromatography $(\mathrm{DCM} / \mathrm{MeOH}=50: 1$ to $20: 1)$, then removing solvent to give the desired catalyst.

\section{(b) Reaction optimization}

Table S1. Optimization of the Reaction Conditions ${ }^{a}$ 

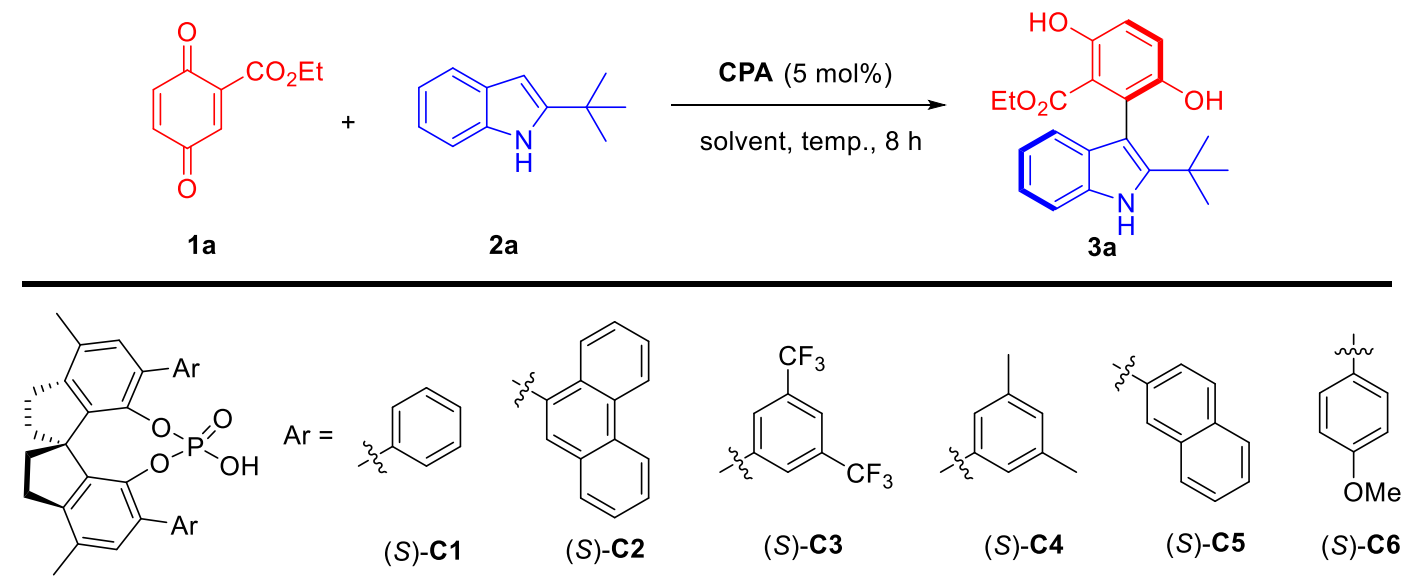

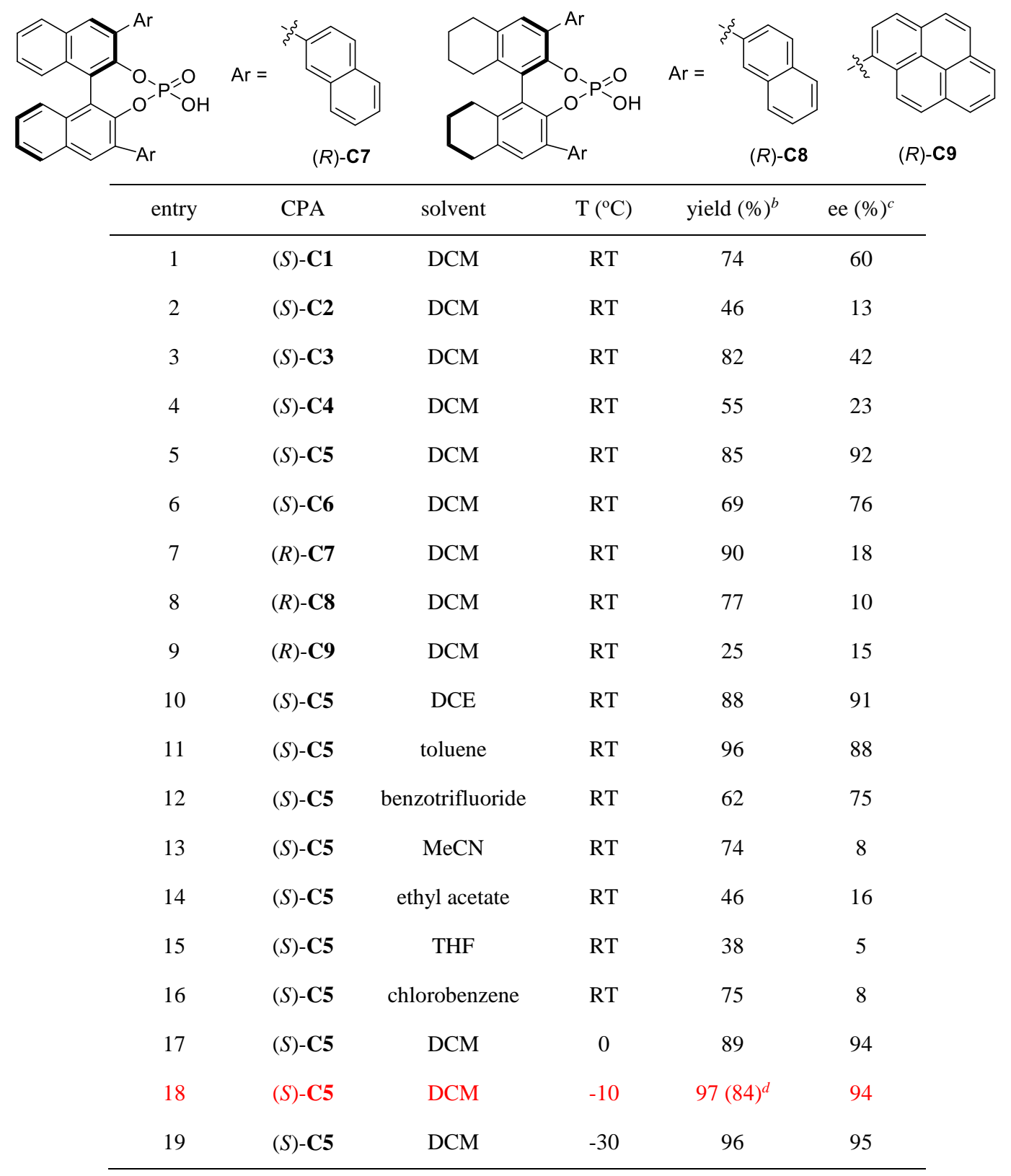

${ }^{a}$ Reaction conditions: 1a $(0.10 \mathrm{mmol}), \mathbf{2 a}(0.12 \mathrm{mmol}), \mathbf{C P A}(5 \mathrm{~mol} \%)$, solvent $(2.0 \mathrm{~mL}), 8 \mathrm{~h}$, 
RT, unless noted otherwise. ${ }^{b}$ Determined by ${ }^{1} \mathrm{HNMR}$ analysis of the crude reaction mixture with 4-Bromo-N,N-dimethylaniline as an internal standard. ${ }^{c}$ Determined by HPLC analysis on a chiral stationary phase. ${ }^{d}$ Yield of isolated product.

\section{(c) General procedure for enantioselective synthesis of 3a-3p}

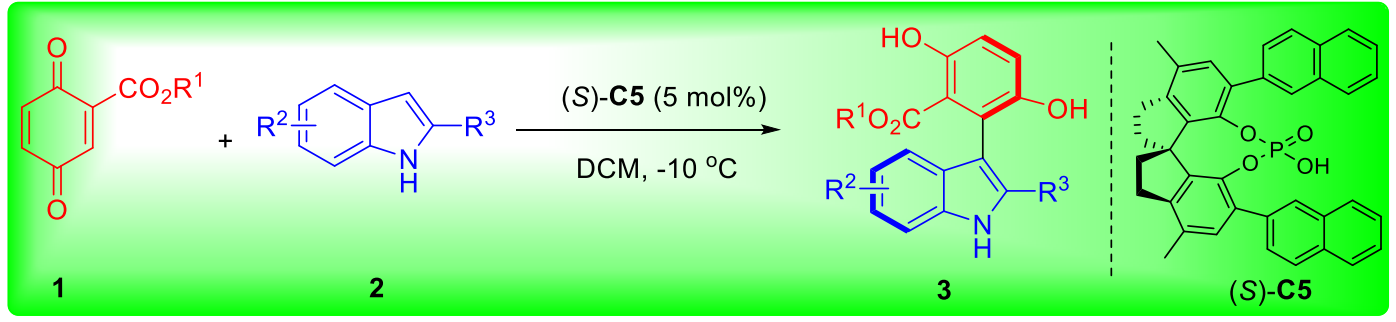

Quinone $1(0.2 \mathrm{mmol})$ dissolved in $1.0 \mathrm{~mL}$ of DCM was added ta solution of chiral phosphoric acid CPA $(5 \mathrm{~mol} \%)$ and $2(0.24 \mathrm{mmol})$ in DCM $(3.0 \mathrm{~mL})$ at $-10{ }^{\circ} \mathrm{C}$, the resulting mixture was stirred at $-10{ }^{\circ} \mathrm{C}$. Confirming completetion of the reaction by TLC, the mixture was concentrated and purified by column chromatography on silica-gel to give products 3 . 


\section{Reaction optimization of $3 q-3 u$ and their synthetic procedures}

\section{(a) Synthesis of chiral phosphoric acids}

Synthesis of other chiral phosphoric acids (CPA 9-19) were prepared according to the literature method. ${ }^{7}$

\section{(b) Reaction optimization}

Table S2. Chiral phosphoric acids, solvent, and temperature effect screening ${ }^{a}$<smiles>O=C1C=C(I)C(=O)C=C1I</smiles>

10<smiles>Cc1cc2ccccc2[nH]1</smiles>

$\underset{\text { solvent, temp. }}{\stackrel{\text { CPA }(15 \mathrm{~mol} \%)}{\longrightarrow}}$

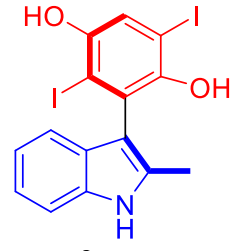

$3 q$<smiles>O=P(O)(Oc1c(Br)cc2c(c1-c1cc(Br)cc3c1CCCC3)CCCC2)Oc1c(Br)cc2ccc3cccc4ccc1c2c34</smiles>

$(R)-\mathbf{C 9}$<smiles>[131I]c1c2ccccc2cc2ccccc12</smiles>

(R)-C10<smiles>Cc1cc(Br)c2c3c1CCC31CCc3c(C)cc(Br)c(c31)OP(=O)(O)O2</smiles><smiles>[Te]=Cc1ccc2ccc3cccc4ccc1c2c34</smiles>

(S)-C11<smiles>O=P(O)(O)Oc1c(Br)cc2ccccc2c1-c1c(Br)cc2ccccc2c1Br</smiles><smiles>[Al][Te]=[Te]</smiles>

(R)-C12<smiles>C[14c]1ccc(Cl)cc1</smiles>

(R)-C13<smiles>[Y]c1cc2ccccc2c2ccccc12</smiles>

(R)-C14

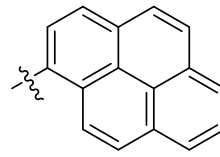

(R)-C15

\begin{tabular}{cccccc}
\hline entry & CPA & solvent & T $\left({ }^{\circ} \mathrm{C}\right)$ & yield $(\%)^{b}$ & ee $(\%)^{c}$ \\
\hline 1 & $(R)-\mathbf{C 9}$ & $\mathrm{DCM}$ & $\mathrm{RT}$ & 85 & 80 \\
2 & $(R)-\mathbf{C 1 0}$ & $\mathrm{DCM}$ & $\mathrm{RT}$ & 60 & 70 \\
3 & $(S)$-C11 & $\mathrm{DCM}$ & $\mathrm{RT}$ & 12 & 13 \\
4 & $(R)$-C12 & $\mathrm{DCM}$ & $\mathrm{RT}$ & 31 & 20 \\
5 & $(R)-\mathbf{C 1 3}$ & $\mathrm{DCM}$ & $\mathrm{RT}$ & 37 & 45 \\
6 & $(R)-\mathbf{C 1 4}$ & $\mathrm{DCM}$ & $\mathrm{RT}$ & 45 & 59 \\
7 & $(R)-\mathbf{C 1 5}$ & $\mathrm{DCM}$ & $\mathrm{RT}$ & 92 & 36 \\
8 & $(R)-\mathbf{C 9}$ & $\mathrm{CHCl}$ & $\mathrm{RT}$ & 90 & 77 \\
9 & $(R)-\mathbf{C 9}$ & $\mathrm{DCE}_{3}$ & $\mathrm{RT}$ & 91 & 81 \\
10 & $(R)-\mathbf{C 9}$ & toluene & $\mathrm{RT}$ & 88 & 55 \\
11 & $(R)-\mathbf{C 9}$ & $\mathrm{CCl}_{4}$ & $\mathrm{RT}$ & 89 & 81 \\
\hline
\end{tabular}




\begin{tabular}{cccccc}
\hline 12 & $(R)-\mathbf{C 9}$ & DCE & -10 & 90 & 72 \\
13 & $(R)-\mathbf{C 9}$ & DCE & 0 & 91 & 74 \\
14 & $(R)-\mathbf{C 9}$ & DCE & 40 & 90 & 70 \\
\hline
\end{tabular}

${ }^{a}$ Reaction was carried out with 1e $(0.05 \mathrm{mmol}), 2 \mathbf{i}(0.075 \mathrm{mmol})$, and CPA $(15 \mathrm{~mol} \%)$ in 2.0 $\mathrm{mL}$ of solvent for $1.5 \mathrm{~h}$ at $25{ }^{\circ} \mathrm{C}$, unless noted otherwise; ${ }^{b}$ Determined by ${ }^{1} \mathrm{H}$ NMR analysis of the crude reaction mixture with 4-Bromo-N,N-dimethylaniline as an internal standard; ${ }^{c}$ ee values determined by HPLC analysis using a chiral stationary phase.

Table S3. Concentration and additive effect ${ }^{a}$<smiles>O=C1C=C(I)C(=O)C=C1I</smiles><smiles>Cc1cc2ccccc2[nH]1</smiles>

$2 \mathbf{i}$ $1 e$

\begin{tabular}{|c|c|c|c|c|}
\hline entry & volume of DCE (mL) & Additive & yield $(\%)^{b}$ & ee $(\%)^{c}$ \\
\hline 1 & 1 & - & 90 & 77 \\
\hline 2 & 2 & - & 91 & 80 \\
\hline 3 & 4 & - & 91 & 84 \\
\hline 4 & 6 & - & 90 & 86 \\
\hline 5 & 8 & - & 90 & 89 \\
\hline 6 & 10 & - & 91 & 89 \\
\hline $7^{d}$ & 8 & $3 \AA ̊ M S ~(50 m g) ~$ & 91 & 88 \\
\hline $8^{d}$ & 8 & 4Å MS (50 mg) & 90 & 88 \\
\hline $9^{d}$ & 8 & $5 \AA ̊ M S(50$ mg) & 89 & 87 \\
\hline $10^{d}$ & 8 & $3 \AA ̊ \mathrm{MS}(100$ mg) & $92(86)^{e}$ & 90 \\
\hline $11^{d, f}$ & 8 & $3 \AA \mathrm{MS}(100 \mathrm{mg})$ & 86 & 89 \\
\hline
\end{tabular}<smiles>[13CH3][13CH3]</smiles>

DCE, additive, RT

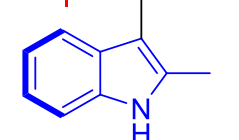

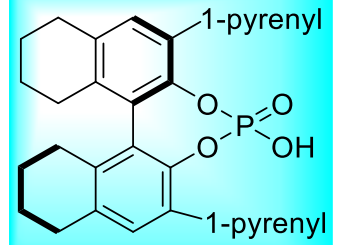

(R)-C9

${ }^{a}$ Reaction was carried out with 1 e $(0.05 \mathrm{mmol}), 2 \mathbf{i}(0.075 \mathrm{mmol})$, and $(R)-\mathbf{C 9}(15 \mathrm{~mol} \%)$ in DCE for $1.5 \mathrm{~h}$ at $25{ }^{\circ} \mathrm{C}$, unless noted otherwise; ${ }^{b}$ Determined by ${ }^{1} \mathrm{H}$ NMR analysis of the crude reaction mixture with 4-Bromo-N,N-dimethylaniline as an internal standard; 'ee values determined by HPLC analysis using a chiral stationary phase. ${ }^{d} \mathbf{1 e}(0.10 \mathrm{mmol})$ and $\mathbf{2} \mathbf{i}(0.12$ mmol). ${ }^{e}$ Yield of isolated product. ${ }^{f}(R)$-C9 $(10 \mathrm{~mol} \%)$

\section{(c) General procedure for enantioselective synthesis of 3q-3u}




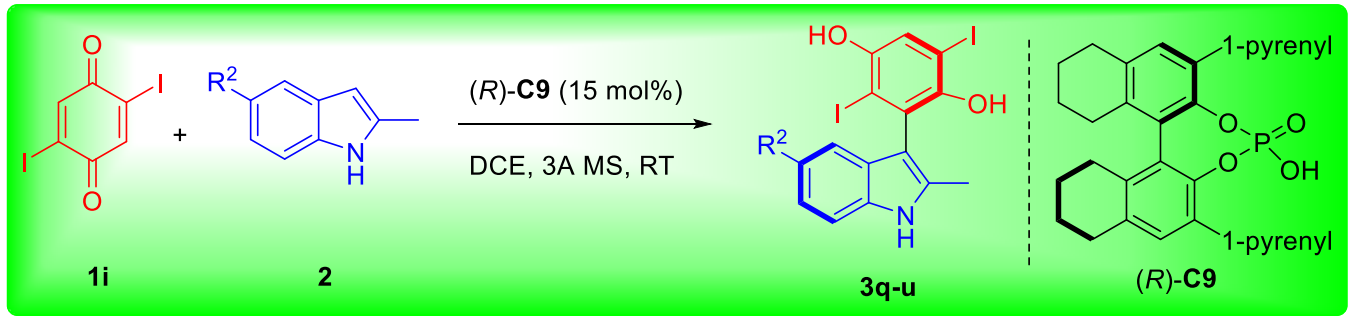

Quinone $1 \mathbf{i}(0.1 \mathrm{mmol})$ dissolved in $1.0 \mathrm{~mL}$ of solvent $\left(\mathrm{DCE}\right.$ or $\left.\mathrm{CCl}_{4}\right)$ was added the mixture of chiral phosphoric acid $(R)$-C9 (15 mol\%), 3A MS (100 mg) and $2(0.12 \mathrm{mmol})$ in $7.0 \mathrm{~mL}$ of solvent ( $\mathrm{DCE}$ or $\mathrm{CCl}_{4}$ ), and the reaction was stirred at room temperature for about 0.5-1.5 hours. Confirming completetion of the reaction by TLC, the mixture was concentrated and purified purified by flash chromatography to give products $\mathbf{3 q - u}$. 
Reaction optimization of $4 \mathrm{a}-4 \mathrm{~g}$ and their synthetic procedures

(a) Initial results for direct synthesis of phenylindoles

\section{Scheme S1}

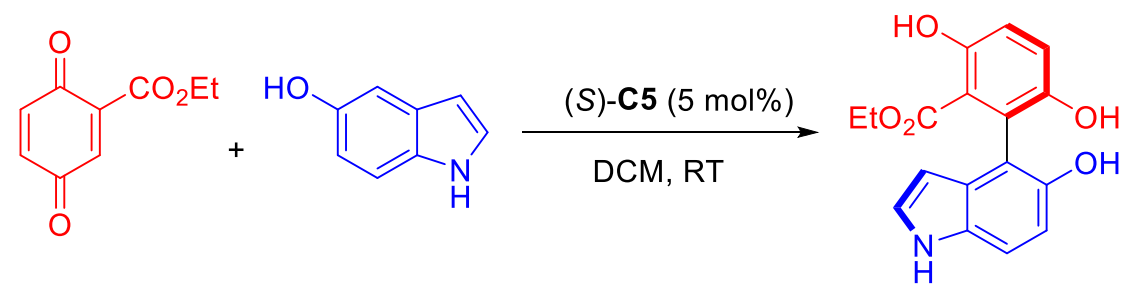

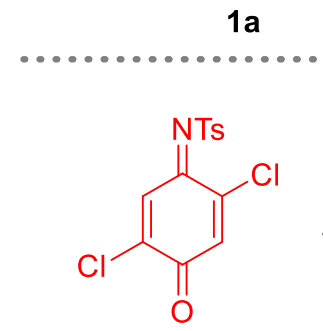

$1 \mathrm{j}$

20

ND

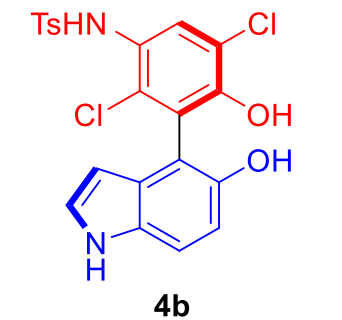

4b

\section{(b) Reaction optimization}

Table S4. Optimization of the Reaction Conditions ${ }^{a}$
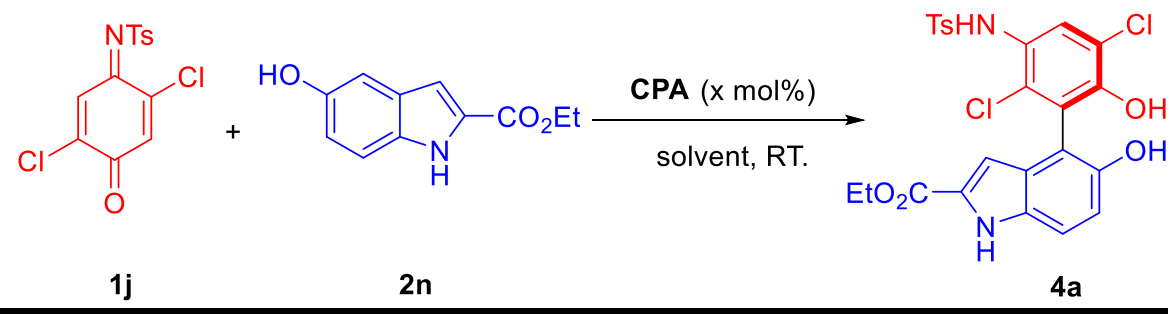<smiles></smiles>

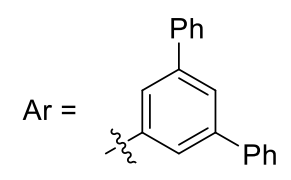

(R)-C16

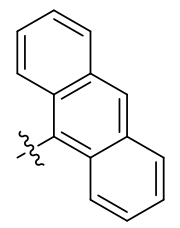

(R)-C17<smiles>Cc1cc(C)cc(C)c1</smiles>

(R)-C18<smiles>Cc1cc(Br)c([C@@H]2CCc3c(C)cc(Br)c(OP(=O)(O)O)c32)c2c1CCC2</smiles><smiles>CC(C)(C)c1ccc(N=[Zn])cc1</smiles>

(S)-C19 


\begin{tabular}{|c|c|c|c|c|c|c|}
\hline entry & $\mathrm{CPA}$ & $\mathrm{x}(\mathrm{mol} \%)$ & solvent & $\mathrm{T}\left({ }^{\circ} \mathrm{C}\right)$ & yield $(\%)^{b}$ & ee $(\%)^{c}$ \\
\hline 1 & $(S)-\mathbf{C 1 1}$ & 10 & DCM & $\mathrm{RT}$ & 88 & 91 \\
\hline 2 & $(R)-\mathbf{C 1 2}$ & 10 & DCM & $\mathrm{RT}$ & 85 & 79 \\
\hline 3 & $(R)-\mathrm{C} 16$ & 10 & DCM & $\mathrm{RT}$ & 45 & 55 \\
\hline 4 & $(R)-\mathbf{C 1 7}$ & 10 & DCM & $\mathrm{RT}$ & 90 & 92 \\
\hline 5 & $(R)-\mathbf{C 1 8}$ & 10 & DCM & $\mathrm{RT}$ & 67 & 45 \\
\hline 6 & $(R)-\mathbf{C 1 9}$ & 10 & DCM & $\mathrm{RT}$ & 80 & 81 \\
\hline 7 & $(R)-\mathbf{C 1 7}$ & 10 & toluene & $\mathrm{RT}$ & 89 & 88 \\
\hline 8 & $(R)-\mathbf{C 1 7}$ & 10 & $\mathrm{CHCl}_{3}$ & RT & 83 & 90 \\
\hline 9 & $(R)-\mathbf{C 1 7}$ & 10 & DCE & $\mathrm{RT}$ & 89 & 92 \\
\hline 10 & $(R)-\mathbf{C 1 7}$ & 10 & $\begin{array}{c}\text { ethyl } \\
\text { acetate }\end{array}$ & $\mathrm{RT}$ & 93 & 91 \\
\hline 11 & $(R)-\mathrm{C} 17$ & 5 & $\begin{array}{c}\text { ethyl } \\
\text { acetate }\end{array}$ & $\mathrm{RT}$ & 93 & 89 \\
\hline 12 & $(R)-\mathbf{C 1 7}$ & 5 & $\begin{array}{c}\text { ethyl } \\
\text { acetate }\end{array}$ & -30 & $97(93)^{d}$ & 91 \\
\hline
\end{tabular}

${ }^{a}$ Reaction conditions: $\mathbf{1 j}(0.10 \mathrm{mmol}), \mathbf{2 n}(0.12 \mathrm{mmol})$, solvent $(1.5 \mathrm{~mL}), 0.5 \mathrm{~h}$, RT, unless noted otherwise. ${ }^{b}$ Determined by ${ }^{1} \mathrm{HNMR}$ analysis of the crude reaction mixture with 4-Bromo-N,N-dimethylaniline as an internal standard. ${ }^{c}$ Determined by HPLC analysis on a chiral stationary phase. ${ }^{d}$ Yield of isolated product.

\section{(c) General procedure for enantioselective synthesis of $\mathbf{4 a - 4 g}$}

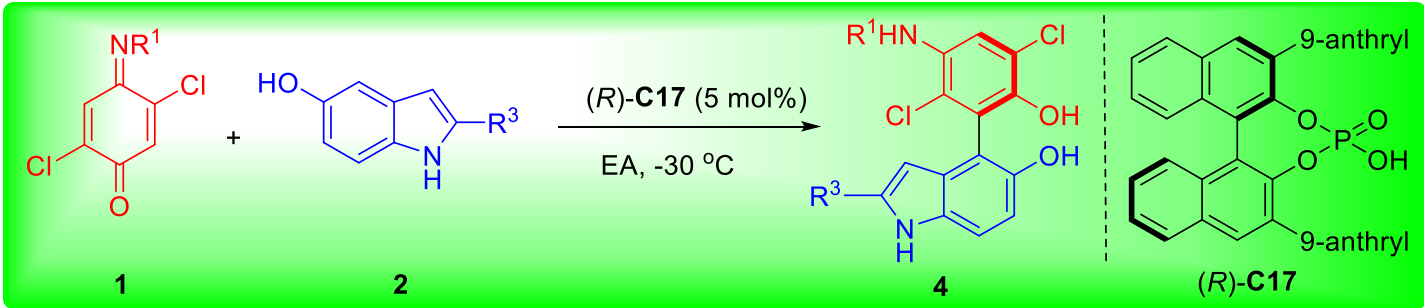

Quinone $1(0.2 \mathrm{mmol})$ dissolved in $1.0 \mathrm{~mL}$ of EA was added the mixture of $(R)$-C17 (5 mol\%) and $2(0.24 \mathrm{mmol})$ in $2.0 \mathrm{~mL}$ of EA at $-30{ }^{\circ} \mathrm{C}$, and the reaction was stirred at at $-30{ }^{\circ} \mathrm{C}$ until the transformation was completed. Solvent was removed under reduced pressure and the crude residue was purified by column chromatography on silica-gel to give product 4 . 


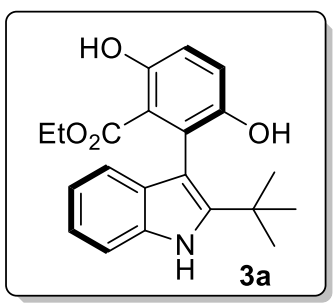

(R)-ethyl 2-(2-(tert-butyl)-1H-indol-3-yl)-3,6-dihydroxybenzoate

White solid; Yield: $59 \mathrm{mg}, 84 \%$ (petroleum ether/DCM $=2: 1)$; ee $=94 \% .[\alpha]_{\mathrm{D}}^{26}=-85.0(\mathrm{c}=$ $\left.0.2, \mathrm{CHCl}_{3}\right)$.

HPLC analysis: HPLC DAICEL CHIRALCEL IA, hexane/isopropanol $=90 / 10,0.8 \mathrm{~mL} / \mathrm{min}$, $\lambda=214 \mathrm{~nm}, \mathrm{t}_{\mathrm{R}}($ minor $)=7.7 \mathrm{~min}, \mathrm{t}_{\mathrm{R}}($ major $)=21.8 \mathrm{~min}$.

${ }^{1}$ H NMR (400 MHz, CDCl $) \delta 10.87(\mathrm{~s}, 1 \mathrm{H}), 8.20(\mathrm{~s}, 1 \mathrm{H}), 7.33(\mathrm{~d}, J=8.1 \mathrm{~Hz}, 1 \mathrm{H}), 7.16(\mathrm{dd}$, $J=11.2,5.1 \mathrm{~Hz}, 2 \mathrm{H}), 7.03(\mathrm{~m}, 3 \mathrm{H}), 4.98(\mathrm{~s}, 1 \mathrm{H}), 3.92-3.67(\mathrm{~m}, 2 \mathrm{H}), 1.26(\mathrm{~s}, 9 \mathrm{H}), 0.35(\mathrm{t}, J$ $=7.1 \mathrm{~Hz}, 3 \mathrm{H})$.

${ }^{13}$ C NMR (100 MHz, $\left.\mathbf{C D C l}_{3}\right) \delta=171.00,156.45,147.74,143.59,134.86,129.85,122.22$, $122.01,121.59,120.19,118.84,118.61,113.36,110.22$, 104.70, 60.80, 33.43, 30.16, 12.62.

HRMS (ESI) Calcd for $\mathrm{C}_{21} \mathrm{H}_{24} \mathrm{NO}_{4}^{+}[\mathrm{M}+\mathrm{H}]^{+}$354.1700; Found: 354.1696.

Chiral HPLC spectrum of racemic 3a DAD1 D, Sig=214,4 Ref=360,100 (C:ICHEM32I...LDL-076-IA-9010-08-30MIN 2019-01-16 17-37-26IIIl-076-4-rac.D)

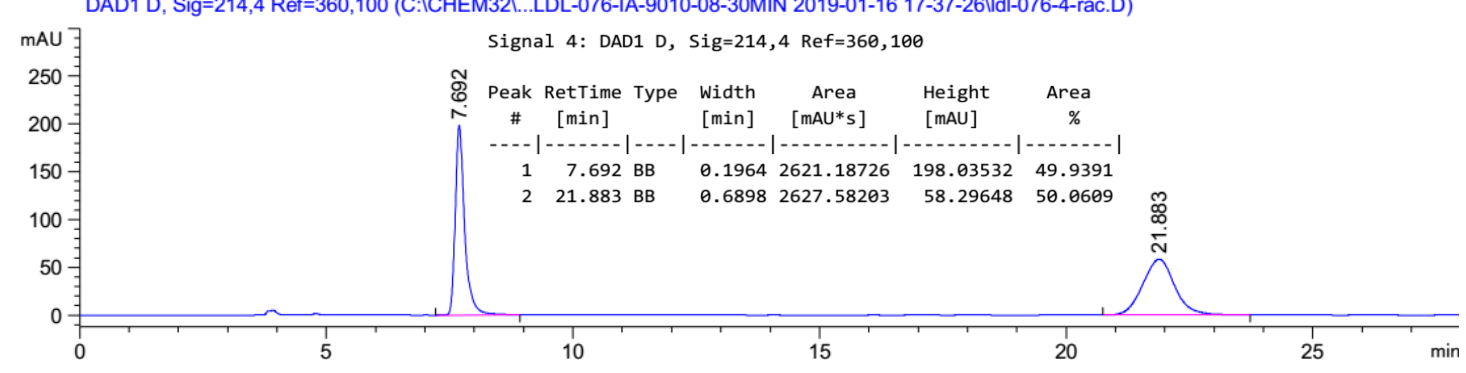

Chiral HPLC spectrum of $(R)-\mathbf{3 a}$

DAD1 D, Sig=214,4 Ref=360,100 (C:ICHEM32I...76-IA-9010-08-30MIN 2019-01-16 17-37-26UIdl-076-4-shouxing.D)

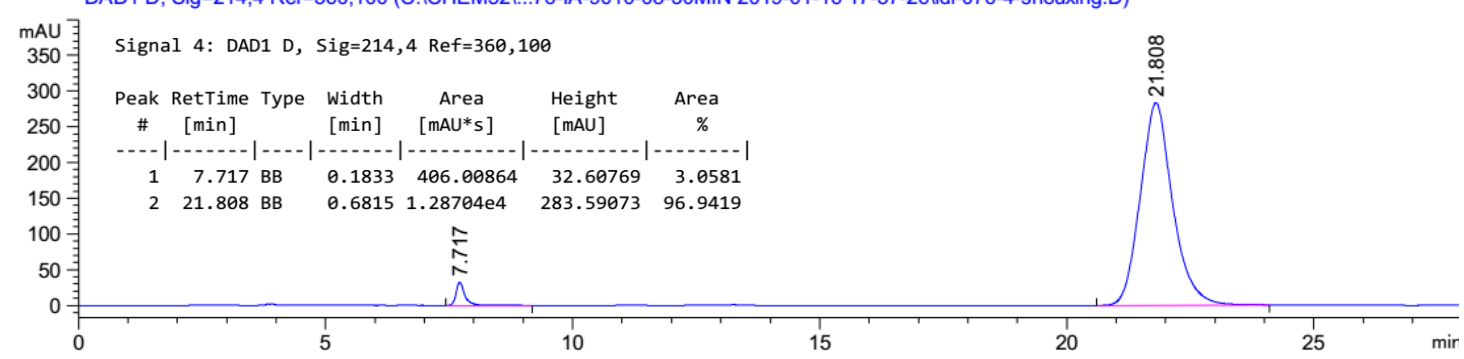




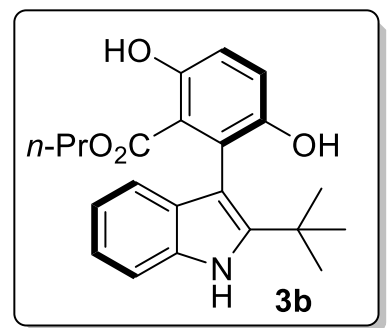

(R)-propyl 2-(2-(tert-butyl)-1H-indol-3-yl)-3,6-dihydroxybenzoate

White solid; Yield: $54 \mathrm{mg}, 73 \%$ (petroleum ether/DCM $=3: 1)$; ee $=94 \%$; $[\alpha]_{\mathrm{D}}^{26}=-60.0(\mathrm{c}=$ $\left.0.2, \mathrm{CHCl}_{3}\right)$.

HPLC analysis: HPLC DAICEL CHIRALCEL IA, hexane/isopropanol $=90 / 10,0.8 \mathrm{~mL} / \mathrm{min}$, $\lambda=214 \mathrm{~nm}, \mathrm{t}_{\mathrm{R}}($ minor $)=7.4 \mathrm{~min}, \mathrm{t}_{\mathrm{R}}($ major $)=17.9 \mathrm{~min}$.

${ }^{1}$ H NMR (400 MHz, CDCl $) \delta 10.94(\mathrm{~s}, 1 \mathrm{H}), 8.22(\mathrm{~s}, 1 \mathrm{H}), 7.33$ (d, $\left.J=8.1 \mathrm{~Hz}, 1 \mathrm{H}\right), 7.20-$ $7.12(\mathrm{~m}, 2 \mathrm{H}), 7.09-6.98(\mathrm{~m}, 3 \mathrm{H}), 4.94(\mathrm{~s}, 1 \mathrm{H}), 3.82(\mathrm{~m}, 1 \mathrm{H}), 3.68(\mathrm{~m}, 1 \mathrm{H}), 1.25(\mathrm{~s}, 9 \mathrm{H}), 0.82$ (m, 1H), $0.68(\mathrm{~m}, 1 \mathrm{H}), 0.25(\mathrm{t}, J=7.4 \mathrm{~Hz}, 3 \mathrm{H})$.

${ }^{13}$ C NMR (100 MHz, CDCl $) \delta=171.33,156.45,147.71,143.54,134.85,129.67,122.28$, $122.00,121.46,120.24,118.85,118.64,113.44,110.32,104.65,66.97,33.39,30.17,20.89$, 9.83 .

HRMS (ESI) Calcd for $\mathrm{C}_{22} \mathrm{H}_{26} \mathrm{NO}_{4}^{+}[\mathrm{M}+\mathrm{H}]^{+} 368.1856$; Found: 368.1848 .

Chiral HPLC spectrum of racemic $\mathbf{3 b}$

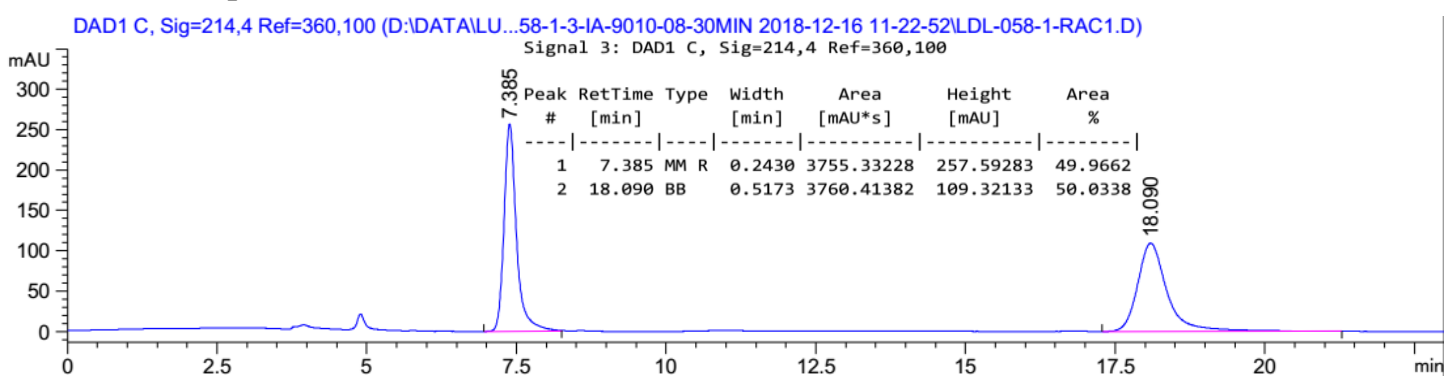

Chiral HPLC spectrum of $(R)-\mathbf{3 b}$

DAD1 C, Sig=214,4 Ref=360,100 (D:IDATAILU...LDL-058-1-3-IA-9010-08-30MIN 2018-12-16 11-22-52ILDL-058-1.D)

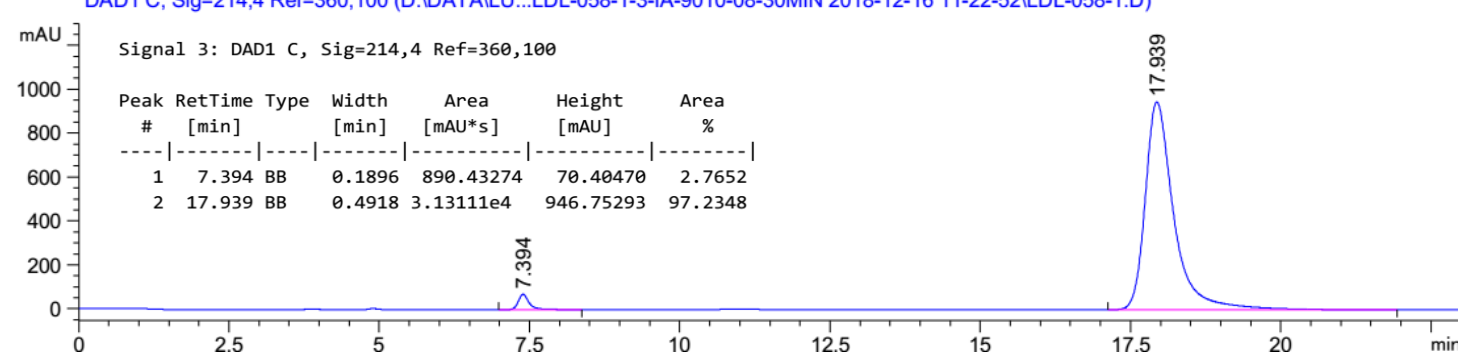




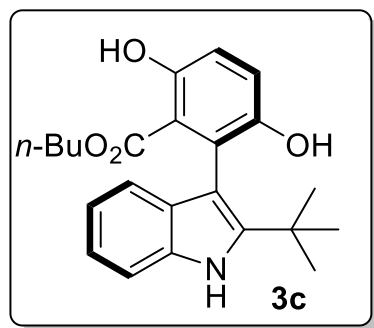

(R)-butyl 2-(2-(tert-butyl)-1H-indol-3-yl)-3,6-dihydroxybenzoate

White solid; Yield: $55 \mathrm{mg}, 72 \%$ (petroleum ether/DCM $=3: 1)$; ee $=95 \%$; $[\alpha]_{\mathrm{D}}^{26}=-65.0(\mathrm{c}=$ $\left.0.2, \mathrm{CHCl}_{3}\right)$.

HPLC analysis: HPLC DAICEL CHIRALCEL IA, hexane/isopropanol $=90 / 10,0.8 \mathrm{~mL} / \mathrm{min}$, $\lambda=214 \mathrm{~nm}, \mathrm{t}_{\mathrm{R}}($ minor $)=7.2 \mathrm{~min}, \mathrm{t}_{\mathrm{R}}($ major $)=16.7 \mathrm{~min}$.

${ }^{1}$ H NMR (400 MHz, CDCl $) \delta 10.95$ (s, 1H), $8.22(\mathrm{~s}, 1 \mathrm{H}), 7.33$ (d, $\left.J=8.1 \mathrm{~Hz}, 1 \mathrm{H}\right), 7.21-$ $7.12(\mathrm{~m}, 2 \mathrm{H}), 7.10-6.98(\mathrm{~m}, 3 \mathrm{H}), 4.92(\mathrm{~s}, 1 \mathrm{H}), 3.90(\mathrm{~m}, 1 \mathrm{H}), 3.81-3.60(\mathrm{~m}, 1 \mathrm{H}), 1.25(\mathrm{~s}$, 9H), $0.85-0.71(\mathrm{~m}, 1 \mathrm{H}), 0.70-0.45(\mathrm{~m}, 6 \mathrm{H})$.

${ }^{13}$ C NMR (100 MHz, CDCl $) \delta=171.39,156.45,147.71,143.52,134.84,129.64,122.28$, $122.00,121.44,120.27,118.83,118.64,113.44,110.34,104.66,65.23,33.39,30.17,29.68$, $18.63,13.65$.

HRMS (ESI) Calcd for $\mathrm{C}_{23} \mathrm{H}_{28} \mathrm{NO}_{4}^{+}[\mathrm{M}+\mathrm{H}]^{+} 382.2013$; Found: 382.2003 .

Chiral HPLC spectrum of racemic 3c

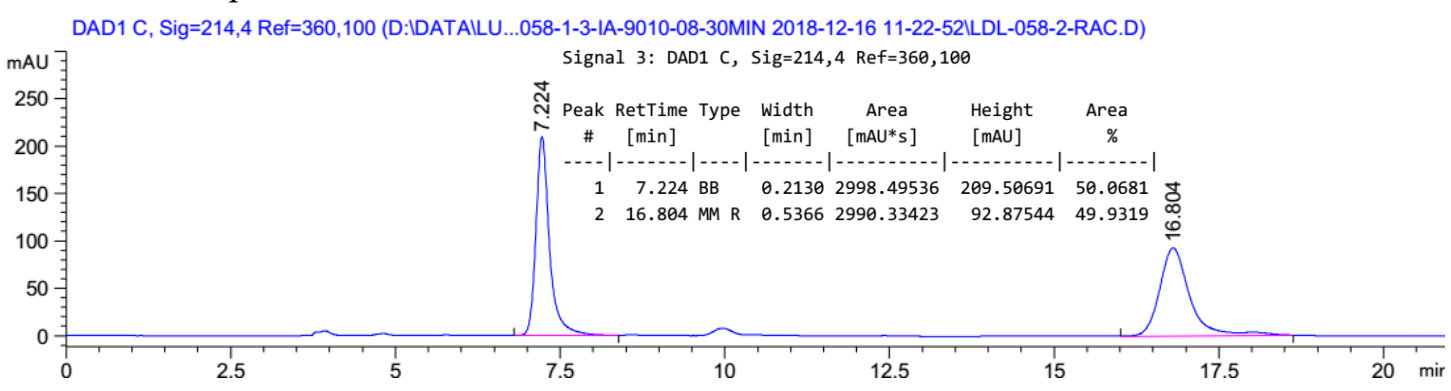

Chiral HPLC spectrum of $(R)-\mathbf{3 c}$

DAD1 C, Sig=214,4 Ref=360,100 (D:IDATAILU...LDL-058-1-3-IA-9010-08-30MIN 2018-12-16 11-22-52ILDL-058-2.D)

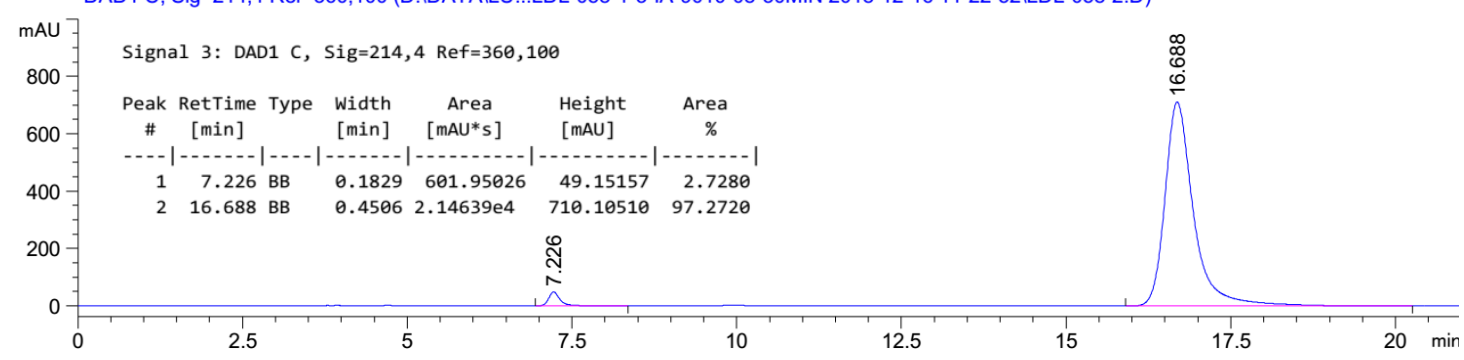




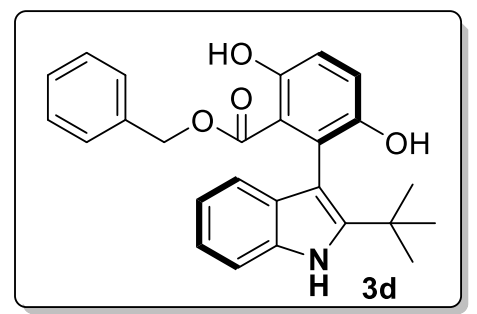

(R)-benzyl 2-(2-(tert-butyl)-1H-indol-3-yl)-3,6-dihydroxybenzoate

White solid; Yield: $66 \mathrm{mg}, 80 \%$ (petroleum ether/DCM $=4: 3$ ); ee $=89 \%$; $[\alpha]_{\mathrm{D}}^{26}=-15.0(\mathrm{c}=$ $\left.0.2, \mathrm{CHCl}_{3}\right)$.

HPLC analysis: HPLC DAICEL CHIRALCEL IA, hexane/isopropanol $=90 / 10,0.8 \mathrm{~mL} / \mathrm{min}$, $\lambda=214 \mathrm{~nm}, \mathrm{t}_{\mathrm{R}}($ minor $)=8.5 \mathrm{~min}, \mathrm{t}_{\mathrm{R}}($ major $)=15.0 \mathrm{~min}$.

${ }^{1}$ H NMR (400 MHz, CDCl $) 10.89$ (s, 1H), 7.85 (s, 1H), 7.24 (s, 1H), $7.21-7.11$ (m, 3H), $7.03(\mathrm{~m}, 5 \mathrm{H}), 6.40(\mathrm{~d}, J=7.4 \mathrm{~Hz}, 2 \mathrm{H}), 4.85(\mathrm{~s}, 1 \mathrm{H}), 4.78(\mathrm{~d}, \mathrm{~J}=2.1 \mathrm{~Hz}, 2 \mathrm{H}), 1.06(\mathrm{~s}, 9 \mathrm{H})$.

${ }^{13} \mathrm{C}$ NMR (100 MHz, $\left.\mathbf{C D C l}_{3}\right) \delta=171.06,156.70,147.67,143.64,134.85,134.20,129.44$, $128.66,128.20,128.13,122.25,122.19,121.56,120.26,118.72,118.67,113.07,110.60$, 104.22, 67.44, 33.25, 29.98 .

HRMS (ESI) Calcd for $\mathrm{C}_{26} \mathrm{H}_{26} \mathrm{NO}_{4}^{+}[\mathrm{M}+\mathrm{H}]^{+}$416.1856; Found: 416.1853 .

Chiral HPLC spectrum of racemic 3d

DAD1 C, Sig=214,4 Ref=360,100 (D:IDATAILU...058-1-3-IA-9010-08-30MIN 2018-12-16 11-22-52ILDL-058-3-RAC.D)

mAU Signal 3: DAD1 C, Sig $=214,4$ Ref $=360,100$

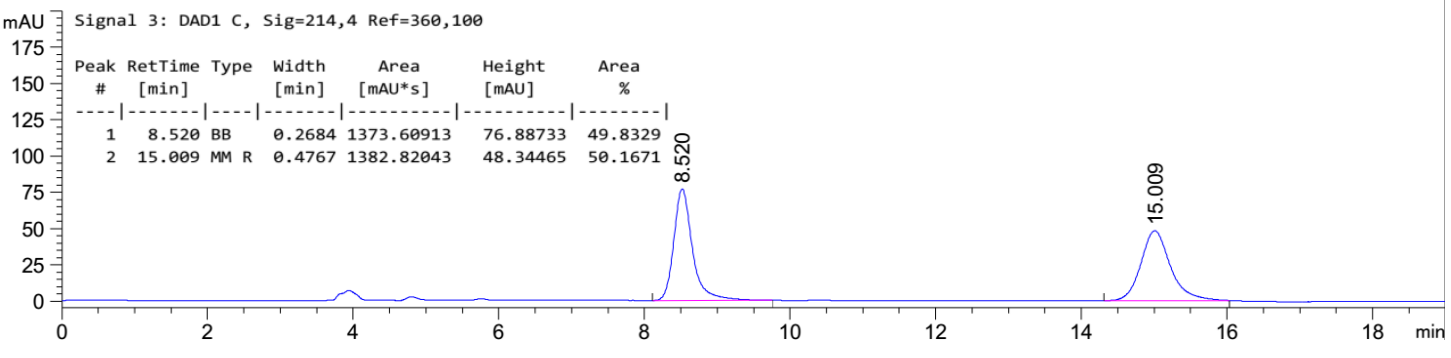

Chiral HPLC spectrum of $(R)-\mathbf{3 d}$

DAD1 C, Sig=214,4 Ref=360,100 (D:IDATAILU...LDL-058-1-3-IA-9010-08-30MIN 2018-12-16 11-22-52ILDL-058-3.D)

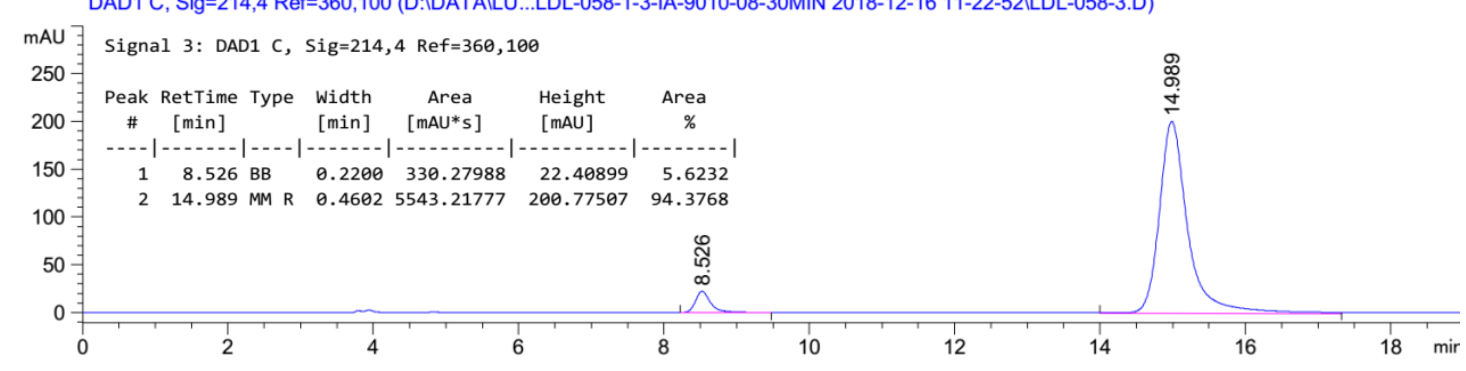




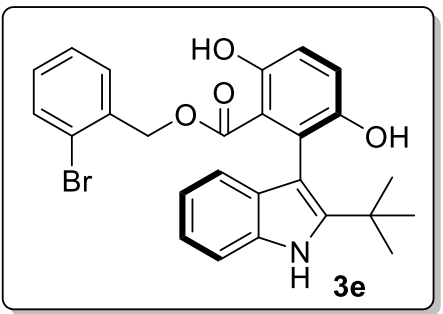

(R)-2-bromobenzyl 2-(2-(tert-butyl)-1H-indol-3-yl)-3,6-dihydroxybenzoate

White solid; Yield: $86 \mathrm{mg}, 87 \%$ (petroleum ether/DCM $=1: 1)$; ee $=90 \% ;[\alpha]_{\mathrm{D}}^{26}=10.0(\mathrm{c}=$ $\left.0.2, \mathrm{CHCl}_{3}\right)$.

HPLC analysis: HPLC DAICEL CHIRALCEL IA, hexane/isopropanol $=90 / 10,0.8 \mathrm{~mL} / \mathrm{min}$, $\lambda=214 \mathrm{~nm}, \mathrm{t}_{\mathrm{R}}($ minor $)=8.5 \mathrm{~min}, \mathrm{t}_{\mathrm{R}}($ major $)=14.0 \mathrm{~min}$.

${ }^{1}$ H NMR (400 MHz, $\left.\mathbf{C D C l}_{3}\right) \delta 10.84(\mathrm{~s}, 1 \mathrm{H}), 7.92(\mathrm{~s}, 1 \mathrm{H}), 7.34(\mathrm{dd}, J=8.0,0.9 \mathrm{~Hz}, 1 \mathrm{H})$, $7.23-7.11(\mathrm{~m}, 3 \mathrm{H}), 7.09-6.98(\mathrm{~m}, 4 \mathrm{H}), 6.89(\mathrm{td}, J=7.5,1.0 \mathrm{~Hz}, 1 \mathrm{H}), 6.07(\mathrm{dd}, J=7.6,1.5$ Hz, 1H), 4.98 (s, 2H), $4.86(\mathrm{~s}, 1 \mathrm{H}), 1.15$ (s, 9H).

${ }^{13}$ C NMR (100 MHz, $\left.\mathbf{C D C l}_{3}\right) \delta=170.93,156.73,147.70,143.72,134.76,133.75,132.42$, $130.02,129.53,129.29,127.30,123.27,122.42,122.28,121.45,120.36,118.78,118.74$, $112.99,110.56,104.19,66.65,33.32,30.10$.

HRMS (ESI) Calcd for $\mathrm{C}_{26} \mathrm{H}_{25} \mathrm{BrNO}_{4}^{+}[\mathrm{M}+\mathrm{H}]^{+}$494.0961; Found: 494.0953.

Chiral HPLC spectrum of racemic $\mathbf{3 e}$ DAD1 C, Sig=214,4 Ref=360,100 (D:IDATAILU...61-1-4-IA-9010-08-35MIN 2018-12-18 14-41-28LLDL-061-1-RAC2.D)

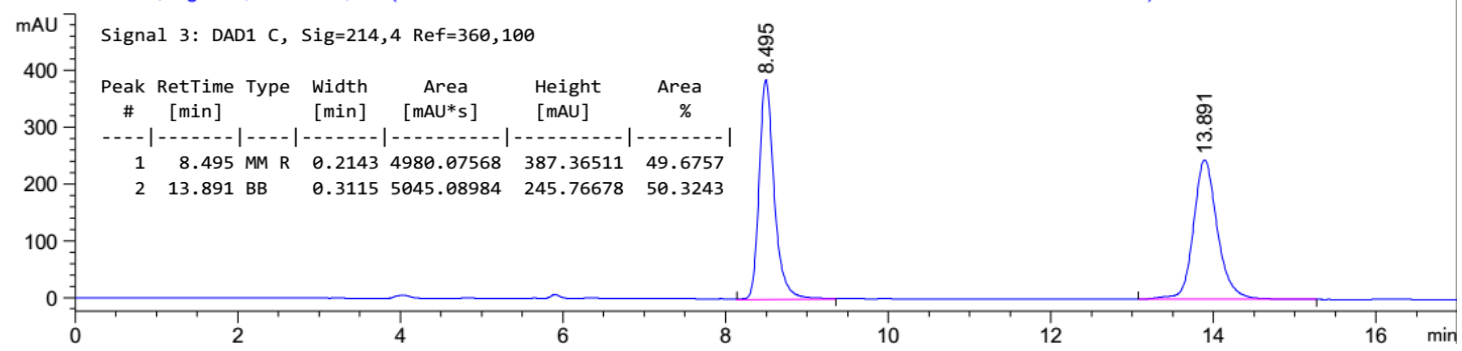

Chiral HPLC spectrum of $(R)-\mathbf{3 e}$ DAD1 C, Sig=214,4 Ref=360,100 (D:IDATAILU...LDL-061-1-4-IA-9010-08-35MIN 2018-12-18 14-41-28ILDL-061-1.D)

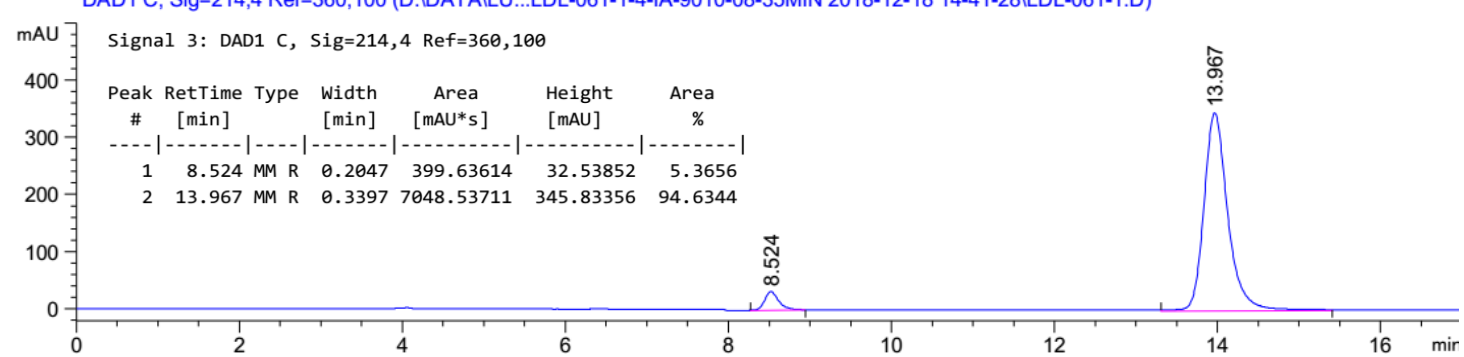




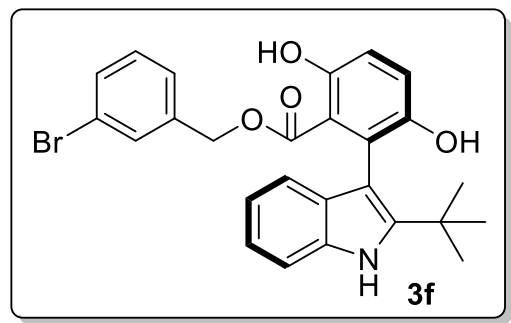

(R)-3-bromobenzyl 2-(2-(tert-butyl)-1H-indol-3-yl)-3,6-dihydroxybenzoate

White solid; Yield: $86 \mathrm{mg}, 87 \%$ (petroleum ether/DCM $=1: 1)$; ee $=90 \%$; $[\alpha]_{\mathrm{D}}^{26}=-25.0(\mathrm{c}=$ $\left.0.2, \mathrm{CHCl}_{3}\right)$.

HPLC analysis: HPLC DAICEL CHIRALCEL IA, hexane/isopropanol $=90 / 10,0.8 \mathrm{~mL} / \mathrm{min}$, $\lambda=214 \mathrm{~nm}, \mathrm{t}_{\mathrm{R}}($ minor $)=8.9 \mathrm{~min}, \mathrm{t}_{\mathrm{R}}($ major $)=13.9 \mathrm{~min}$.

${ }^{1}$ H NMR (400 MHz, CDCl $) \delta 10.74(\mathrm{~s}, 1 \mathrm{H}), 8.04(\mathrm{~s}, 1 \mathrm{H}), 7.35(\mathrm{~d}, J=8.0 \mathrm{~Hz}, 1 \mathrm{H}), 7.31(\mathrm{~d}, J=$ $8.1 \mathrm{~Hz}, 1 \mathrm{H}), 7.20-7.13(\mathrm{~m}, 2 \mathrm{H}), 7.02(\mathrm{t}, J=6.8 \mathrm{~Hz}, 3 \mathrm{H}), 6.95(\mathrm{t}, J=7.8 \mathrm{~Hz}, 1 \mathrm{H}), 6.62(\mathrm{~s}, 1 \mathrm{H})$, $6.47(\mathrm{~d}, J=7.6 \mathrm{~Hz}, 1 \mathrm{H}), 4.98-4.76(\mathrm{~m}, 2 \mathrm{H}), 4.59$ (d, $J=11.9 \mathrm{~Hz}, 1 \mathrm{H}), 1.09$ (s, 9H).

${ }^{13}$ C NMR (100 MHz, CDCl $) \delta=170.84,156.58,147.74,143.62,136.37,134.54,131.69$, $131.51,129.96,129.40,127.65,122.39,122.29,122.15,121.45,120.31,118.72,118.57$, $113.04,110.80,104.28,66.40,33.25,29.99$.

HRMS (ESI) Calcd for $\mathrm{C}_{26} \mathrm{H}_{25} \mathrm{BrNO}_{4}^{+}[\mathrm{M}+\mathrm{H}]^{+}$494.0961; Found: 494.0952.

Chiral HPLC spectrum of racemic $\mathbf{3 f}$

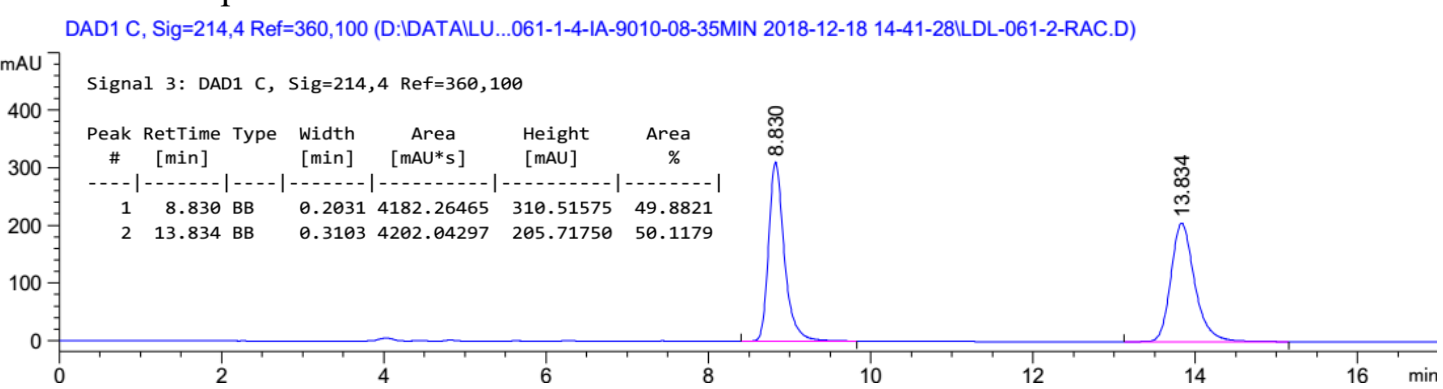

Chiral HPLC spectrum of $(R)-\mathbf{3 f}$ DAD1 C, Sig=214,4 Ref=360,100 (D:IDATAILU...LDL-061-1-4-IA-9010-08-35MIN 2018-12-18 14-41-28ILDL-061-2.D)

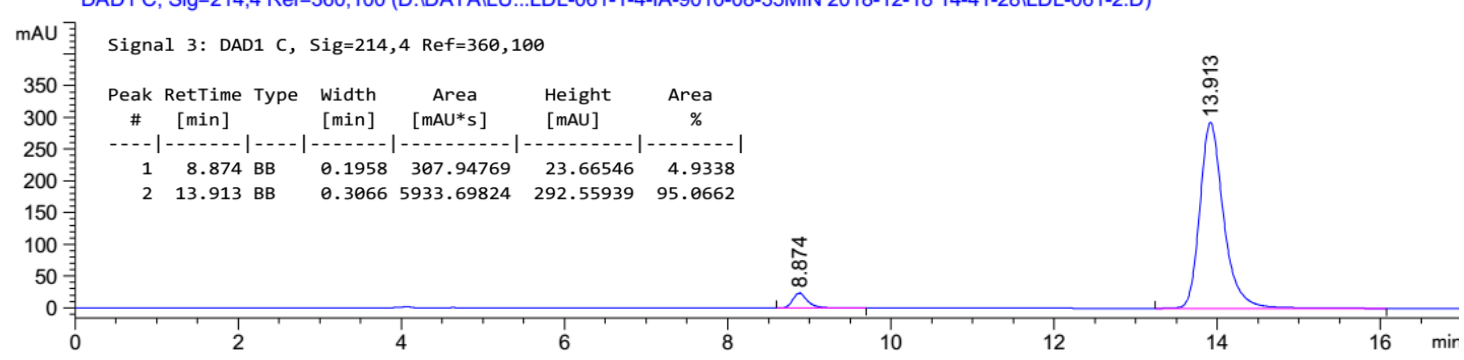




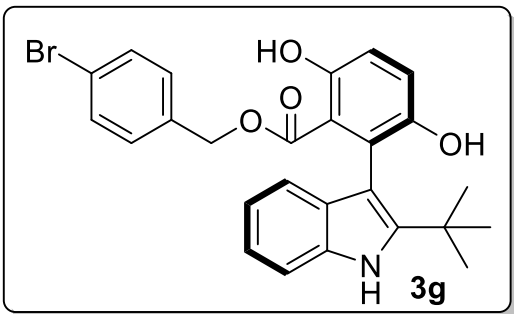

(R)-4-bromobenzyl 2-(2-(tert-butyl)-1H-indol-3-yl)-3,6-dihydroxybenzoate

White solid; Yield: $78 \mathrm{mg}, 79 \%$ (petroleum ether/DCM $=2: 1)$; ee $=90 \%$; $[\alpha]_{\mathrm{D}}^{26}=-30.0(\mathrm{c}=$ $\left.0.2, \mathrm{CHCl}_{3}\right)$.

HPLC analysis: HPLC DAICEL CHIRALCEL IA, hexane/isopropanol $=90 / 10,0.8 \mathrm{~mL} / \mathrm{min}$, $\lambda=214 \mathrm{~nm}, \mathrm{t}_{\mathrm{R}}($ minor $)=8.8 \mathrm{~min}, \mathrm{t}_{\mathrm{R}}($ major $)=15.4 \mathrm{~min}$.

${ }^{1}$ H NMR (400 MHz, CDCl $) \delta 10.85(\mathrm{~s}, 1 \mathrm{H}), 7.94(\mathrm{~s}, 1 \mathrm{H}), 7.25(\mathrm{~d}, J=7.1 \mathrm{~Hz}, 1 \mathrm{H}), 7.21(\mathrm{dd}$, $J=5.4,2.6 \mathrm{~Hz}, 1 \mathrm{H}), 7.19-7.14(\mathrm{~m}, 3 \mathrm{H}), 7.07-6.98(\mathrm{~m}, 3 \mathrm{H}), 6.27(\mathrm{~d}, J=8.3 \mathrm{~Hz}, 2 \mathrm{H}), 4.87$ (s, 1H), $4.82(\mathrm{~d}, J=12.1 \mathrm{~Hz}, 1 \mathrm{H}), 4.68(\mathrm{~d}, J=12.1 \mathrm{~Hz}, 1 \mathrm{H}), 1.11(\mathrm{~s}, 9 \mathrm{H})$.

${ }^{13}$ C NMR (100 MHz, $\left.\mathbf{C D C l}_{3}\right) \delta=170.92,156.71,147.72,143.60,134.78,133.09,132.36$, $131.30,130.15,129.34,122.44,122.26,122.21,121.43,120.28,118.73,118.69,112.87$, $110.58,104.22,66.54,33.28,30.06$.

HRMS (ESI) Calcd for $\mathrm{C}_{26} \mathrm{H}_{25} \mathrm{BrNO}_{4}^{+}[\mathrm{M}+\mathrm{H}]^{+}$494.0961; Found: 494.0953.

Chiral HPLC spectrum of racemic $\mathbf{3 g}$ DAD1 C, Sig=214,4 Ref=360,100 (D:IDATAILU...061-1-4-IA-9010-08-35MIN 2018-12-18 14-41-28LLDL-061-3-RAC.D)

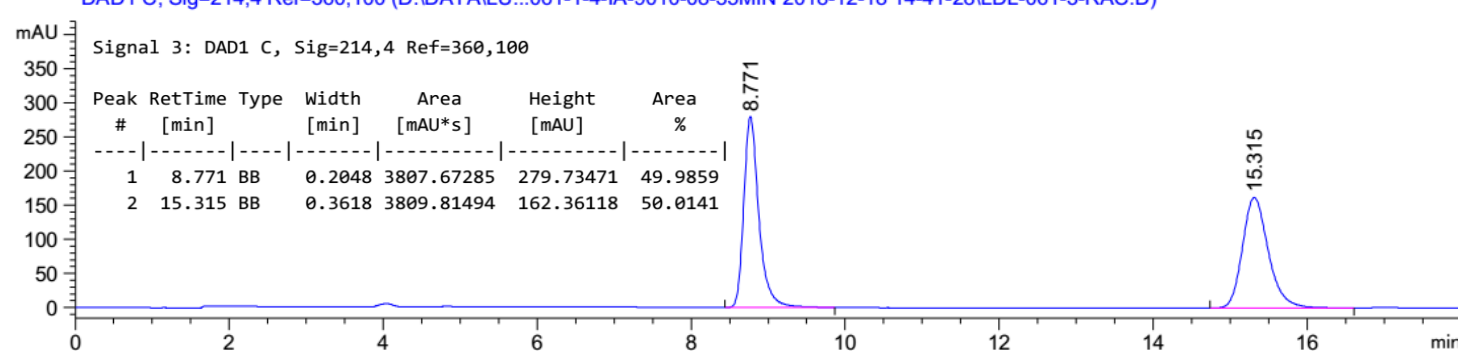

Chiral HPLC spectrum of $(R)-\mathbf{3 g}$

DAD1 C, Sig=214,4 Ref=360,100 (D:IDATAILU...LDL-061-1-4-IA-9010-08-35MIN 2018-12-18 14-41-28ILDL-061-3.D)

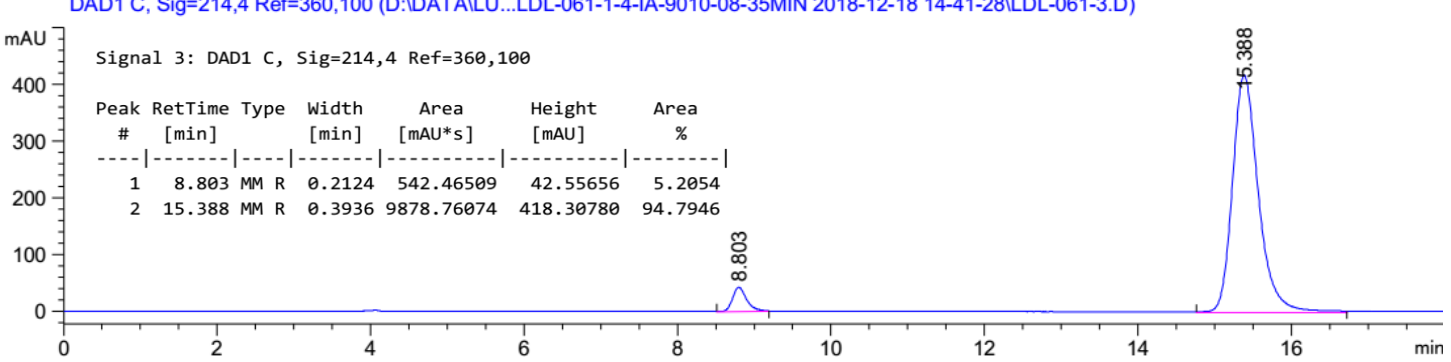




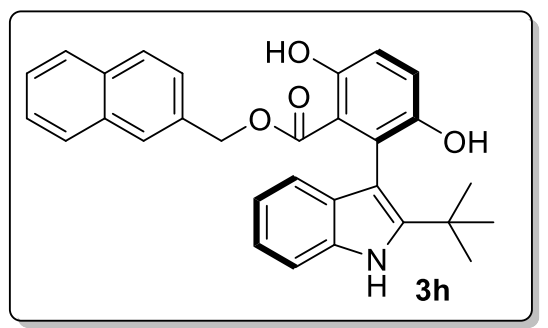

(R)-naphthalen-2-ylmethyl 2-(2-(tert-butyl)-1H-indol-3-yl)-3,6-

dihydroxybenzoate

White solid; Yield: $79 \mathrm{mg}, 85 \%$ (petroleum ether/DCM $=1: 1)$; ee $=88 \%$; $[\alpha]_{\mathrm{D}}^{26}=-25.0(\mathrm{c}=$ $\left.0.2, \mathrm{CHCl}_{3}\right)$.

HPLC analysis: HPLC DAICEL CHIRALCEL IA, hexane/isopropanol $=90 / 10,0.8 \mathrm{~mL} / \mathrm{min}$, $\lambda=214 \mathrm{~nm}, \mathrm{t}_{\mathrm{R}}($ minor $)=9.3 \mathrm{~min}, \mathrm{t}_{\mathrm{R}}($ major $)=13.8 \mathrm{~min}$.

${ }^{1} \mathbf{H}$ NMR (400 MHz, CDCl $) \delta 10.97$ (s, 1H), 7.87 - $7.66(\mathrm{~m}, 3 \mathrm{H}), 7.57$ - 7.48 (m, 3H), 7.22 $-7.11(\mathrm{~m}, 2 \mathrm{H}), 7.12-6.99(\mathrm{~m}, 5 \mathrm{H}), 6.50$ (dd, $J=8.4,1.7 \mathrm{~Hz}, 1 \mathrm{H}), 4.96$ (q, $J=11.8 \mathrm{~Hz}, 2 \mathrm{H})$, $4.91(\mathrm{~s}, 1 \mathrm{H}), 1.04(\mathrm{~s}, 9 \mathrm{H})$.

${ }^{13} \mathrm{C}$ NMR (100 MHz, $\left.\mathbf{C D C l}_{3}\right) \delta=171.01,156.58,147.68,143.57,134.66,133.07,132.90$, $131.56,129.36,128.12,128.09,127.92,127.65,126.37,126.35,126.08,122.22,122.06$, $121.64,120.10,118.57,118.54,113.09,110.47,104.10,67.56,33.13,29.89$.

HRMS (ESI) Calcd for $\mathrm{C}_{30} \mathrm{H}_{28} \mathrm{NO}_{4}^{+}[\mathrm{M}+\mathrm{H}]^{+}$466.2013; Found: 466.2004 .

Chiral HPLC spectrum of racemic $\mathbf{3 h}$

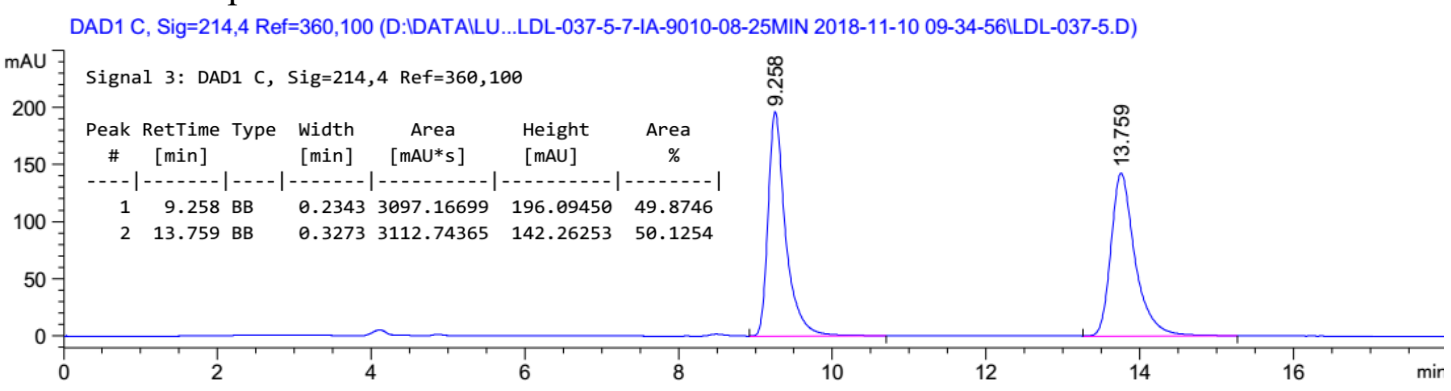

Chiral HPLC spectrum of $(R)-\mathbf{3 h}$

DAD1 C, Sig=214,4 Ref=360,100 (D:IDATAILU...GILDL-037-4-IA-9010-08-25MIN 2018-11-10 11-18-52LLDL-037-4.D)

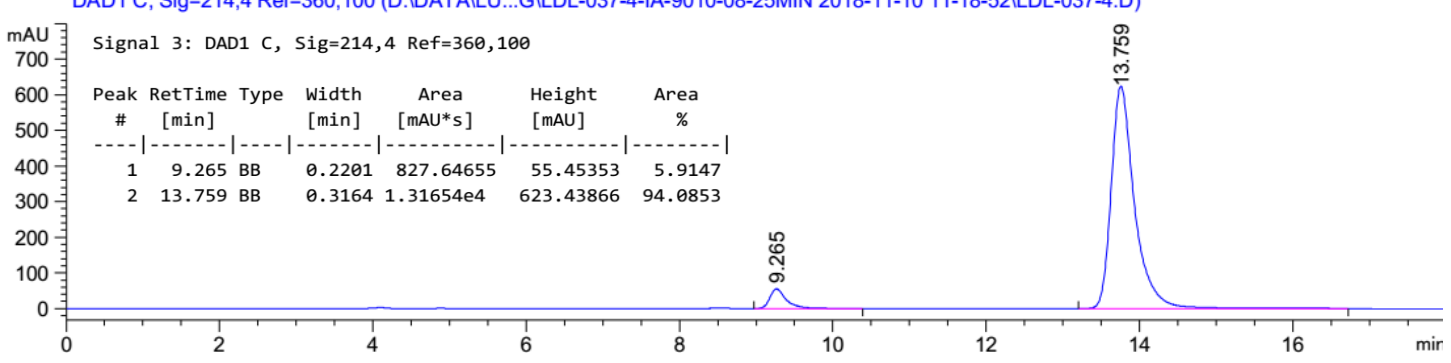




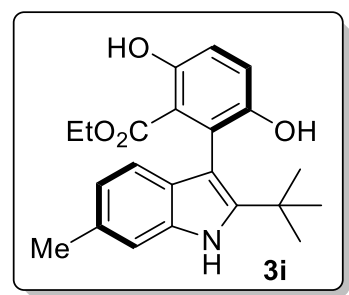

(R)-ethyl 2-(2-(tert-butyl)-5-methoxy-1H-indol-3-yl)-3,6-dihydroxybenzoate

White solid; Yield: $68 \mathrm{mg}, 92 \%$ (petroleum ether/DCM $=2: 1)$; ee $=94 \% ;[\alpha]_{\mathrm{D}}^{26}=-70.0(\mathrm{c}=$ $\left.0.2, \mathrm{CHCl}_{3}\right)$.

HPLC analysis: HPLC DAICEL CHIRALCEL IA, hexane/isopropanol $=90 / 10,0.8 \mathrm{~mL} / \mathrm{min}$, $\lambda=214 \mathrm{~nm}, \mathrm{t}_{\mathrm{R}}($ minor $)=7.9 \mathrm{~min}, \mathrm{t}_{\mathrm{R}}$ (major) $=32.1 \mathrm{~min}$.

${ }^{1}$ H NMR (400 MHz, CDCl 3$) \delta 10.87$ (s, 1H), $8.11(\mathrm{~s}, 1 \mathrm{H}), 7.16$ (d, J = 9.0 Hz, 1H), $7.12(\mathrm{~s}$, 1H), $7.01(\mathrm{~d}, J=9.0 \mathrm{~Hz}, 1 \mathrm{H}), 6.93(\mathrm{~d}, J=8.0 \mathrm{~Hz}, 1 \mathrm{H}), 6.84(\mathrm{dd}, J=8.1,0.9 \mathrm{~Hz}, 1 \mathrm{H}), 5.02(\mathrm{~s}$, 1H), $4.08-3.64(\mathrm{~m}, 2 \mathrm{H}), 2.45(\mathrm{~s}, 3 \mathrm{H}), 1.24(\mathrm{~s}, 9 \mathrm{H}), 0.40(\mathrm{t}, J=7.2 \mathrm{~Hz}, 3 \mathrm{H})$.

${ }^{13}$ C NMR (100 MHz, $\left.\mathbf{C D C l}_{3}\right) \delta=171.04,156.34,147.73,142.80,135.33,131.97,127.63$, $121.89,121.79,118.44,113.37,110.23,104.40,60.79,33.37,30.16,21.84,12.73$.

HRMS (ESI) Calcd for $\mathrm{C}_{22} \mathrm{H}_{26} \mathrm{NO}_{4}^{+}[\mathrm{M}+\mathrm{H}]^{+} 368.1856$; Found: 368.1846 .

Chiral HPLC spectrum of racemic 3i

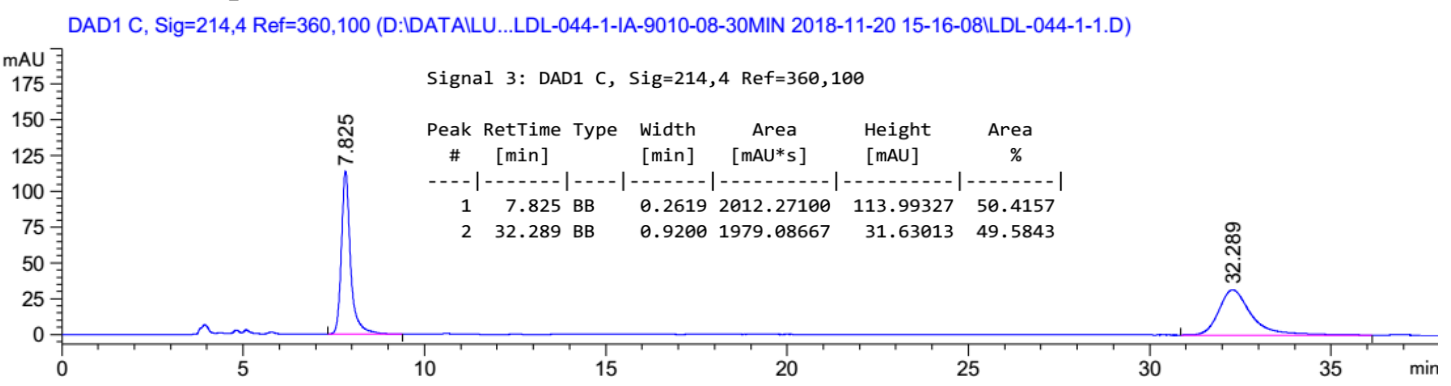

Chiral HPLC spectrum of $(R)-\mathbf{3 i}$

DAD1 C, Sig=214,4 Ref=360,100 (D:IDATAILU...GILDL-044-1-IA-9010-08-30MIN 2018-11-20 15-16-08ILDL-044-1.D)

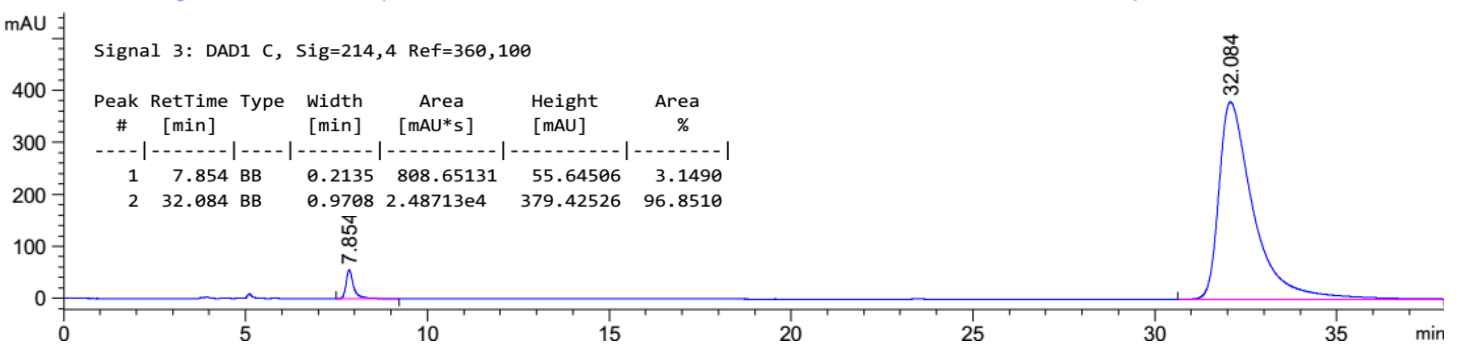




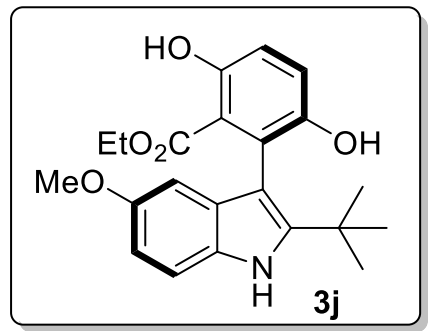

(R)-ethyl 2-(2-(tert-butyl)-5-methoxy-1H-indol-3-yl)-3,6-dihydroxybenzoate

White solid; Yield: $58 \mathrm{mg}, 76 \%$ (petroleum ether/DCM $=2: 1)$; ee $=92 \%$; $[\alpha]_{\mathrm{D}}^{26}=-75.0(\mathrm{c}=$ $\left.0.2, \mathrm{CHCl}_{3}\right)$.

HPLC analysis: HPLC DAICEL CHIRALCEL IA, hexane/isopropanol $=90 / 10,0.8 \mathrm{~mL} / \mathrm{min}$, $\lambda=254 \mathrm{~nm}, \mathrm{t}_{\mathrm{R}}($ minor $)=8.5 \mathrm{~min}, \mathrm{t}_{\mathrm{R}}($ major $)=12.6 \mathrm{~min}$.

${ }^{1}$ H NMR (400 MHz, CDCl 3 ) $\delta 10.87(\mathrm{~s}, 1 \mathrm{H}), 8.07(\mathrm{~s}, 1 \mathrm{H}), 7.23(\mathrm{~d}, J=8.7 \mathrm{~Hz}, 1 \mathrm{H}), 7.18(\mathrm{~d}, J$ $=9.0 \mathrm{~Hz}, 1 \mathrm{H}), 7.03(\mathrm{~d}, J=9.0 \mathrm{~Hz}, 1 \mathrm{H}), 6.81(\mathrm{dd}, J=8.7,2.4 \mathrm{~Hz}, 1 \mathrm{H}), 6.46(\mathrm{~d}, J=2.2 \mathrm{~Hz}, 1 \mathrm{H})$, $5.02(\mathrm{~s}, 1 \mathrm{H}), 3.92-3.75(\mathrm{~m}, 2 \mathrm{H}), 3.71(\mathrm{~s}, 3 \mathrm{H}), 1.23(\mathrm{~s}, 9 \mathrm{H}), 0.40(\mathrm{t}, J=7.1 \mathrm{~Hz}, 3 \mathrm{H})$.

${ }^{13}$ C NMR (100 MHz, CDCl $) \delta=171.01,156.47,154.71,147.78,144.37,130.41,129.81$, $122.03,121.59,118.67,113.41,112.37,111.07,104.59,100.37,60.83,56.01,33.46,30.14$, 12.72 .

HRMS (ESI) Calcd for $\mathrm{C}_{22} \mathrm{H}_{26} \mathrm{NO}_{5}^{+}[\mathrm{M}+\mathrm{H}]^{+} 384.1805$; Found: 384.1797.

Chiral HPLC spectrum of racemic $\mathbf{3 j}$

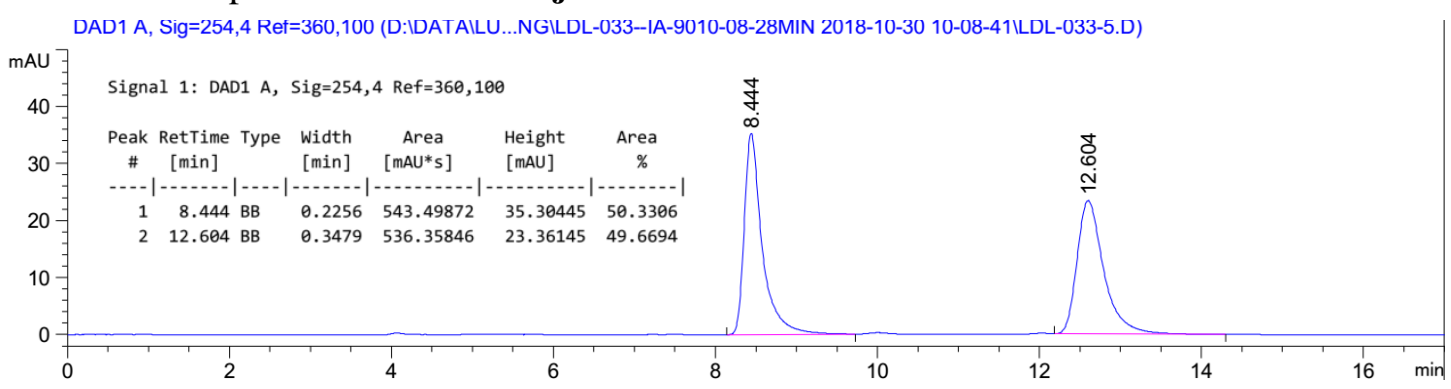

Chiral HPLC spectrum of $(R)-\mathbf{3 j}$

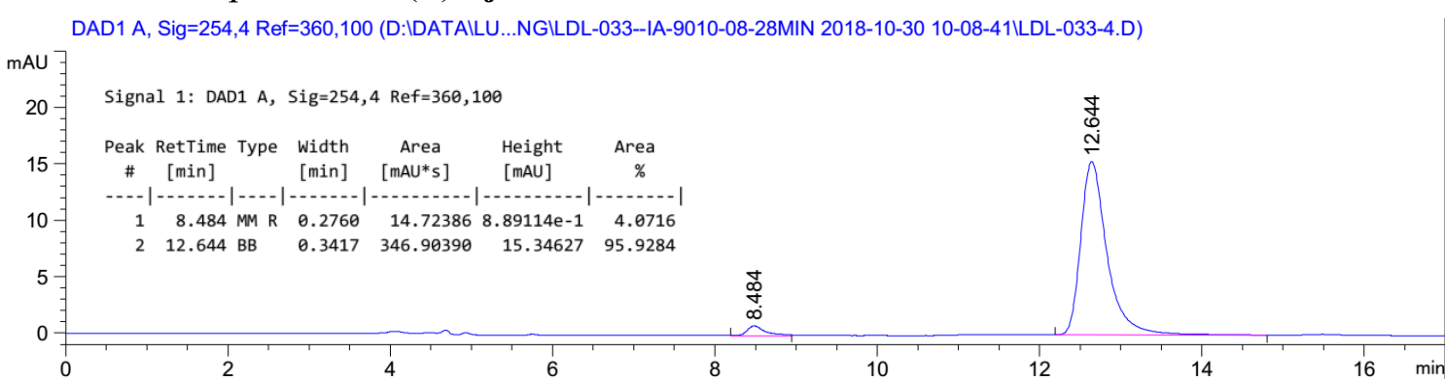




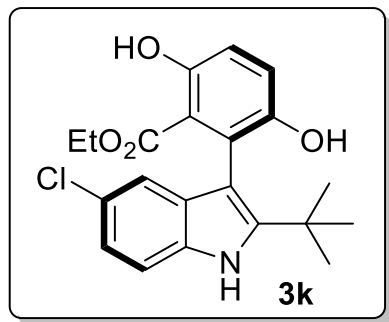

(R)-ethyl 2-(2-(tert-butyl)-5-chloro-1H-indol-3-yl)-3,6-dihydroxybenzoate

White solid; Yield: $63 \mathrm{mg}, 81 \%$ (petroleum ether/DCM $=3: 2)$; ee $=96 \%$; $[\alpha]_{\mathrm{D}}^{26}=-90.0(\mathrm{c}=$ $\left.0.2, \mathrm{CHCl}_{3}\right)$.

HPLC analysis: HPLC DAICEL CHIRALCEL IA, hexane/isopropanol $=90 / 10,0.8 \mathrm{~mL} / \mathrm{min}$, $\lambda=214 \mathrm{~nm}, \mathrm{t}_{\mathrm{R}}($ minor $)=8.1 \mathrm{~min}, \mathrm{t}_{\mathrm{R}}($ major $)=11.1 \mathrm{~min}$.

${ }^{1}$ H NMR (400 MHz, CDCl $) \delta 10.90(\mathrm{~s}, 1 \mathrm{H}), 8.24(\mathrm{~s}, 1 \mathrm{H}), 7.28(\mathrm{~d}, J=2.0 \mathrm{~Hz}, 1 \mathrm{H}), 7.19$ (d, $J$ $=9.0 \mathrm{~Hz}, 1 \mathrm{H}), 7.13(\mathrm{dd}, J=8.5,2.0 \mathrm{~Hz}, 1 \mathrm{H}), 7.06(\mathrm{~s}, 1 \mathrm{H}), 7.04-7.01(\mathrm{~m}, 1 \mathrm{H}), 4.87(\mathrm{~s}, 1 \mathrm{H})$, $4.14-3.51(\mathrm{~m}, 2 \mathrm{H}), 1.26(\mathrm{~s}, 9 \mathrm{H}), 0.41(\mathrm{t}, J=7.1 \mathrm{~Hz}, 3 \mathrm{H})$.

${ }^{13}$ C NMR (100 MHz, CDCl $) \delta=170.80,156.68,147.65,145.26,133.13,131.03,125.98$, $122.56,122.30,120.54,119.08,118.28,113.12,111.37,104.67,60.88,33.51,30.05,12.75$.

HRMS (ESI) Calcd for $\mathrm{C}_{21} \mathrm{H}_{23} \mathrm{ClNO}_{4}{ }^{+}[\mathrm{M}+\mathrm{H}]^{+}$388.1310; Found: 388.1306.

Chiral HPLC spectrum of racemic $\mathbf{3 k}$

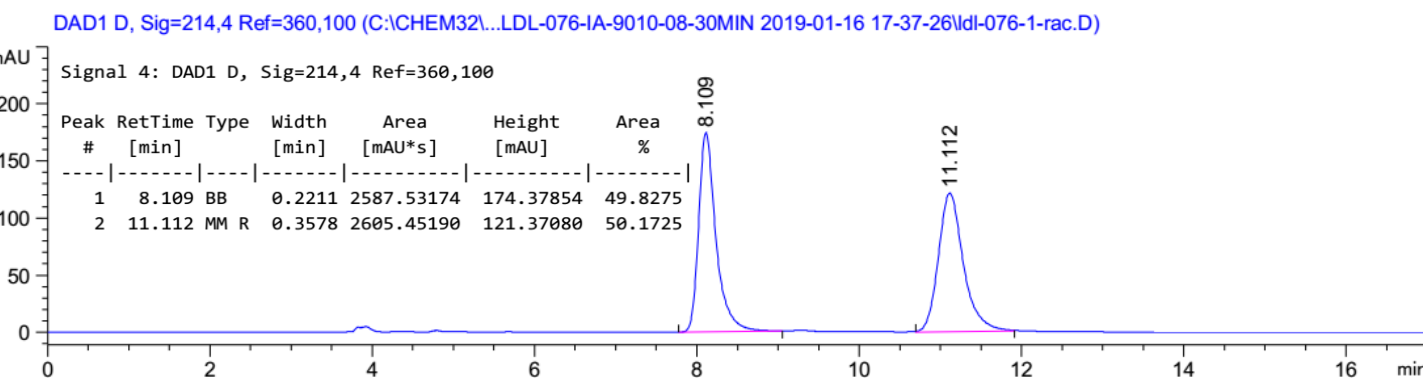

Chiral HPLC spectrum of $(R)-\mathbf{3 k}$ DAD1 D, Sig=214,4 Ref=360,100 (C:ICHEM32I...76-IA-9010-08-30MIN 2019-01-16 17-37-26Udl-076-1-shouxing.D)

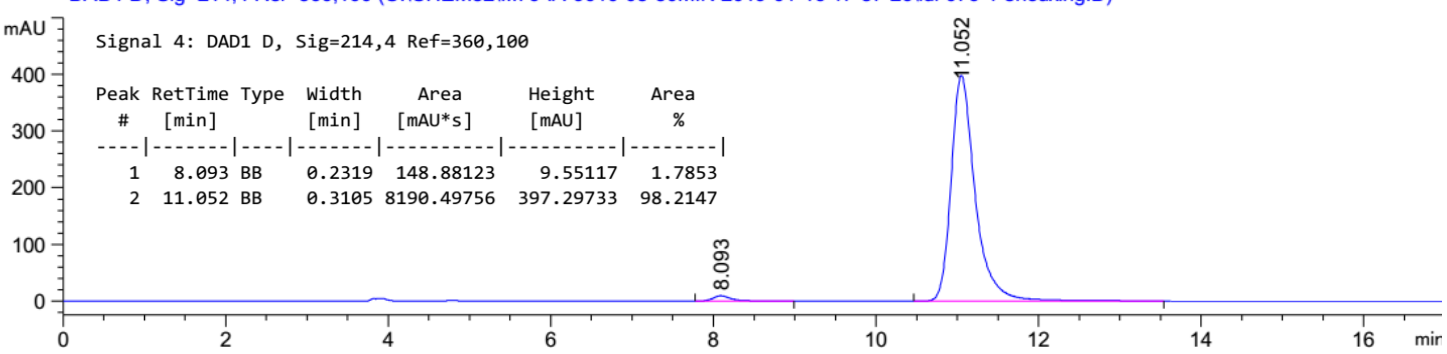




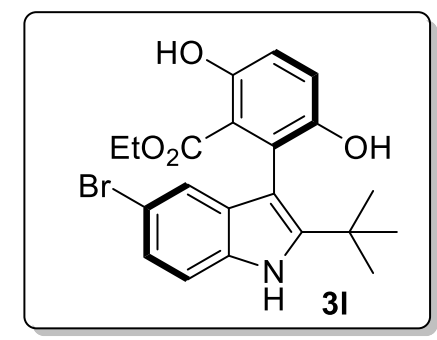

(R)-ethyl 2-(5-bromo-2-(tert-butyl)-1 H-indol-3-yl)-3,6-dihydroxybenzoate

White solid; Yield: $71 \mathrm{mg}, 82 \%$ (petroleum ether/DCM $=1: 1)$; ee $=93 \% ;[\alpha]_{\mathrm{D}}^{26}=-85.0(\mathrm{c}=$ $\left.0.2, \mathrm{CHCl}_{3}\right)$.

HPLC analysis: HPLC DAICEL CHIRALCEL IA, hexane/isopropanol $=90 / 10,0.8 \mathrm{~mL} / \mathrm{min}$, $\lambda=214 \mathrm{~nm}, \mathrm{t}_{\mathrm{R}}($ minor $)=8.0 \mathrm{~min}, \mathrm{t}_{\mathrm{R}}($ major $)=10.8 \mathrm{~min}$

${ }^{1}$ H NMR (400 MHz, CDCl $) \delta 10.90(\mathrm{~s}, 1 \mathrm{H}), 8.28(\mathrm{~s}, 1 \mathrm{H}), 7.31-7.11(\mathrm{~m}, 4 \mathrm{H}), 7.03(\mathrm{~d}, J=$ $9.0 \mathrm{~Hz}, 1 \mathrm{H}), 4.87(\mathrm{~s}, 1 \mathrm{H}), 3.83(\mathrm{~m}, 2 \mathrm{H}), 1.24(\mathrm{~s}, 9 \mathrm{H}), 0.40(\mathrm{t}, J=7.1 \mathrm{~Hz}, 3 \mathrm{H})$.

${ }^{13}$ C NMR (100 MHz, CDCl $) \delta=170.79,156.63,147.66,145.14,133.41,131.61,125.08$, 122.30, 121.28, 120.51, 119.06, 113.41, 113.12, 111.81, 104.50, 60.89, 33.47, 30.03, 12.74 .

HRMS (ESI) Calcd for $\mathrm{C}_{21} \mathrm{H}_{23} \mathrm{BrNO}_{4}^{+}[\mathrm{M}+\mathrm{H}]^{+} 432.0805$; Found: 432.0797.

Chiral HPLC spectrum of racemic 31

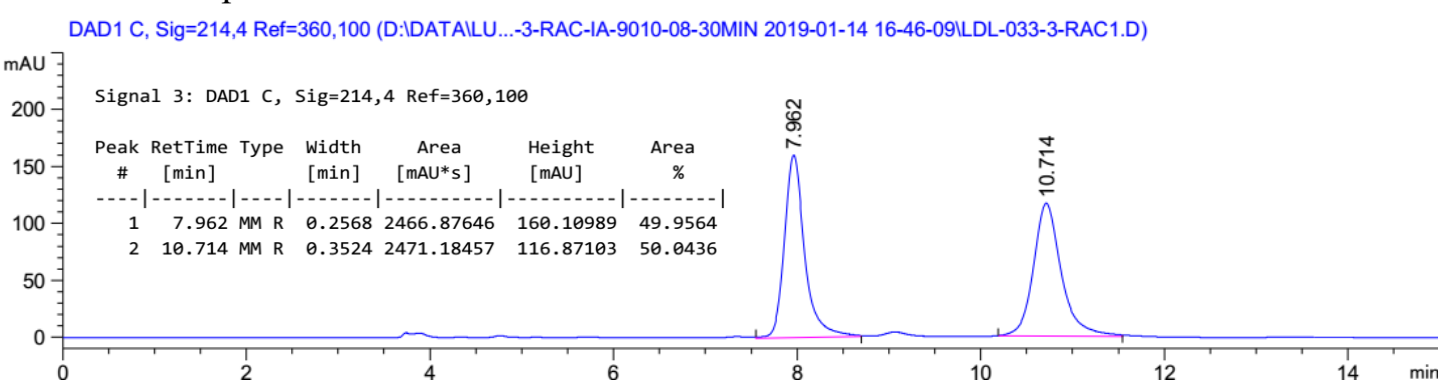

Chiral HPLC spectrum of $(R)-\mathbf{3 l}$

DAD1 C, Sig=214,4 Ref=360,100 (D:IDATAILU...GILDL-033-3-IA-9010-08-30MIN 2019-01-14 09-44-48ILDL-033-3.D)

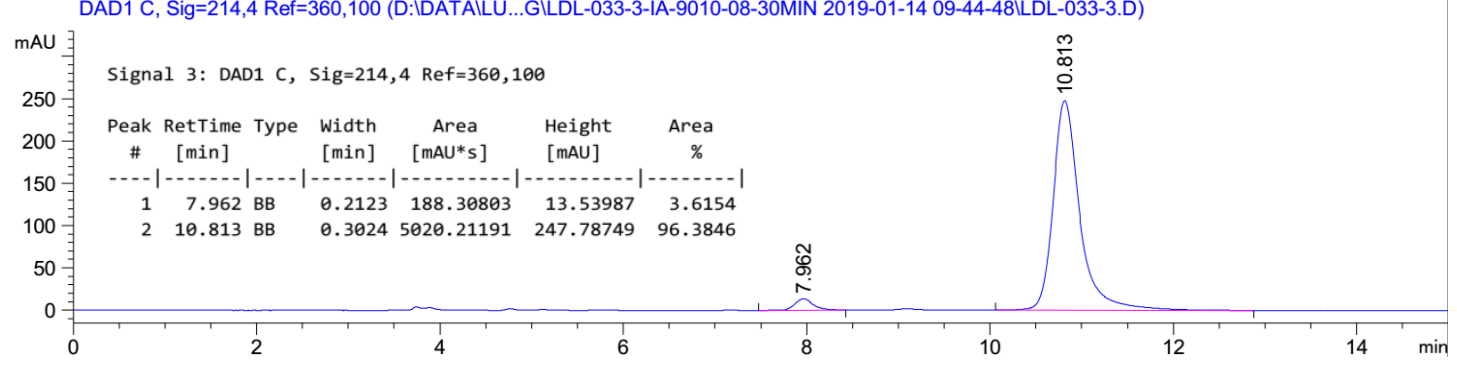




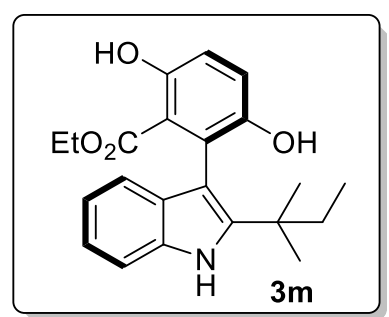

(R)-ethyl 2-(2-(tert-butyl)-5-methoxy-1H-indol-3-yl)-3,6-dihydroxybenzoate

White solid; Yield: $60 \mathrm{mg}, 82 \%$ (petroleum ether/DCM $=2: 1)$; ee $=94 \%$; $[\alpha]_{\mathrm{D}}^{26}=-85.0(\mathrm{c}=$ $\left.0.2, \mathrm{CHCl}_{3}\right)$.

HPLC analysis: HPLC DAICEL CHIRALCEL IA, hexane/isopropanol $=90 / 10,0.8 \mathrm{~mL} / \mathrm{min}$, $\lambda=214 \mathrm{~nm}, \mathrm{t}_{\mathrm{R}}($ minor $)=7.1 \mathrm{~min}, \mathrm{t}_{\mathrm{R}}($ major $)=15.6 \mathrm{~min}$.

${ }^{1}$ H NMR (400 MHz, CDCl $) \delta 10.86(\mathrm{~s}, 1 \mathrm{H}), 8.22(\mathrm{~s}, 1 \mathrm{H}), 7.33(\mathrm{~d}, J=8.0 \mathrm{~Hz}, 1 \mathrm{H}), 7.16(\mathrm{dd}$, $J=8.3,5.9 \mathrm{~Hz}, 2 \mathrm{H}), 7.10-6.96(\mathrm{~m}, 3 \mathrm{H}), 4.97(\mathrm{~s}, 1 \mathrm{H}), 3.98-3.80(\mathrm{~m}, 1 \mathrm{H}), 3.79-3.61(\mathrm{~m}$, $1 \mathrm{H}), 1.62(\mathrm{q}, J=7.4 \mathrm{~Hz}, 2 \mathrm{H}), 1.17(\mathrm{~s}, 6 \mathrm{H}), 0.78(\mathrm{t}, J=7.4 \mathrm{~Hz}, 3 \mathrm{H}), 0.31(\mathrm{t}, J=7.1 \mathrm{~Hz}, 3 \mathrm{H})$.

${ }^{13}$ C NMR (100 MHz, $\left.\mathbf{C D C l}_{3}\right) \delta=171.01,156.30,147.80,142.69,134.94,129.93,122.17$, $121.93,121.59,120.07,118.91,118.56,113.52,110.21,105.56,60.88,37.08,36.17,27.48$, $27.06,12.49,9.40$.

HRMS (ESI) Calcd for $\mathrm{C}_{26} \mathrm{H}_{26} \mathrm{NO}_{4}^{+}[\mathrm{M}+\mathrm{H}]^{+} 368.1856$; Found: 368.1852 .

Chiral HPLC spectrum of racemic $\mathbf{3 m}$

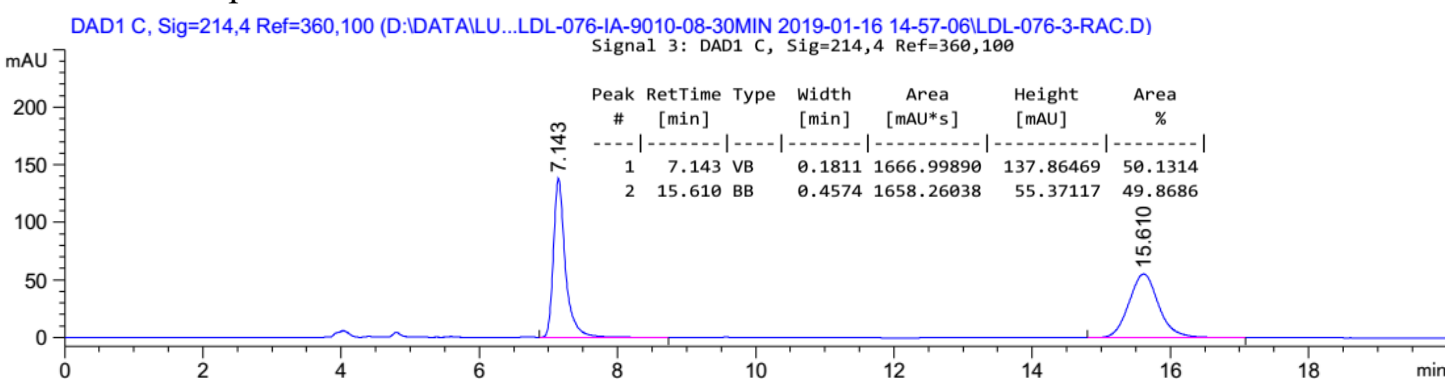

Chiral HPLC spectrum of $(R)-\mathbf{3 m}$ DAD1 C, Sig=214,4 Ref=360,100 (D:IDATAILU...76-IA-9010-08-30MIN 2019-01-16 14-57-06ILDL-076-3-SHOUXING.D)

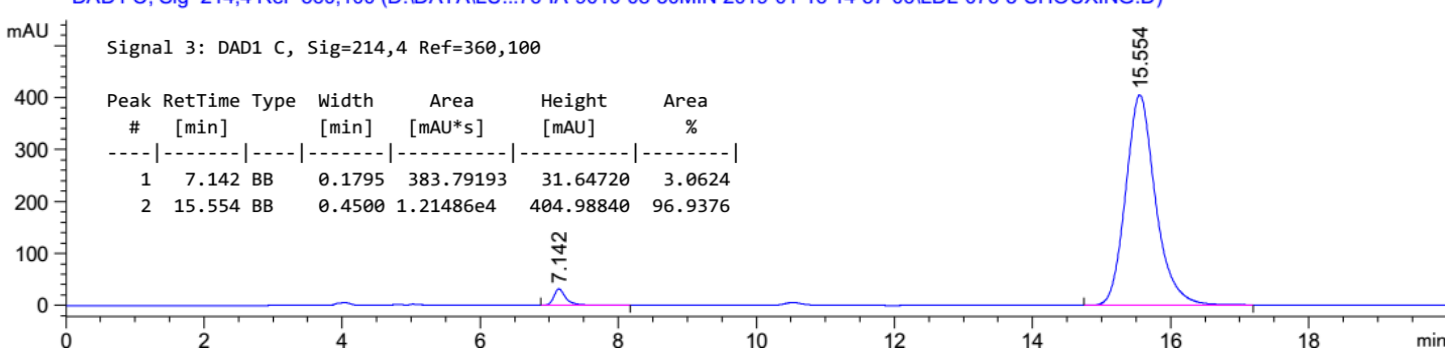




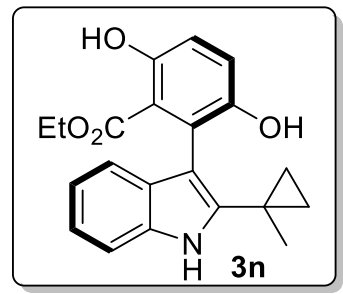

(R)-ethyl 3,6-dihydroxy-2-(2-(1-methylcyclopropyl)-1H-indol-3-yl)benzoate

White solid; Yield: $58 \mathrm{mg}, 83 \%$ (petroleum ether/DCM $=3: 2$ ); ee $=54 \%$.

HPLC analysis: HPLC DAICEL CHIRALCEL 1A, hexane/isopropanol $=90 / 10,0.8 \mathrm{~mL} / \mathrm{min}$, $\lambda=210 \mathrm{~nm}, \mathrm{t}_{\mathrm{R}}($ minor $)=9.2 \mathrm{~min}, \mathrm{t}_{\mathrm{R}}($ major $)=25.3 \mathrm{~min}$.

${ }^{1}$ H NMR (400 MHz, CDCl $) \delta 10.70(\mathrm{~s}, 1 \mathrm{H}), 8.21(\mathrm{~s}, 1 \mathrm{H}), 7.32(\mathrm{~d}, J=8.1 \mathrm{~Hz}, 1 \mathrm{H}), 7.22-$ $7.09(\mathrm{~m}, 3 \mathrm{H}), 7.06-6.99(\mathrm{~m}, 2 \mathrm{H}), 5.02(\mathrm{~s}, 1 \mathrm{H}), 3.77$ (q, J = 7.1 Hz, 2H), $1.32(\mathrm{~s}, 3 \mathrm{H}), 0.85-$ $0.67(\mathrm{~m}, 2 \mathrm{H}), 0.57(\mathrm{~m}, 2 \mathrm{H}), 0.34(\mathrm{t}, J=7.1 \mathrm{~Hz}, 3 \mathrm{H})$.

${ }^{13}$ C NMR (100 MHz, $\left.\mathbf{C D C l}_{3}\right) \delta=170.94,156.15,147.58,139.88,135.28,129.32,122.39$, $122.04,120.31,118.79,118.47,113.23,110.38,106.61,60.84,24.81,14.20,13.87,13.33$, 12.64 .

Chiral HPLC spectrum of racemic 3n

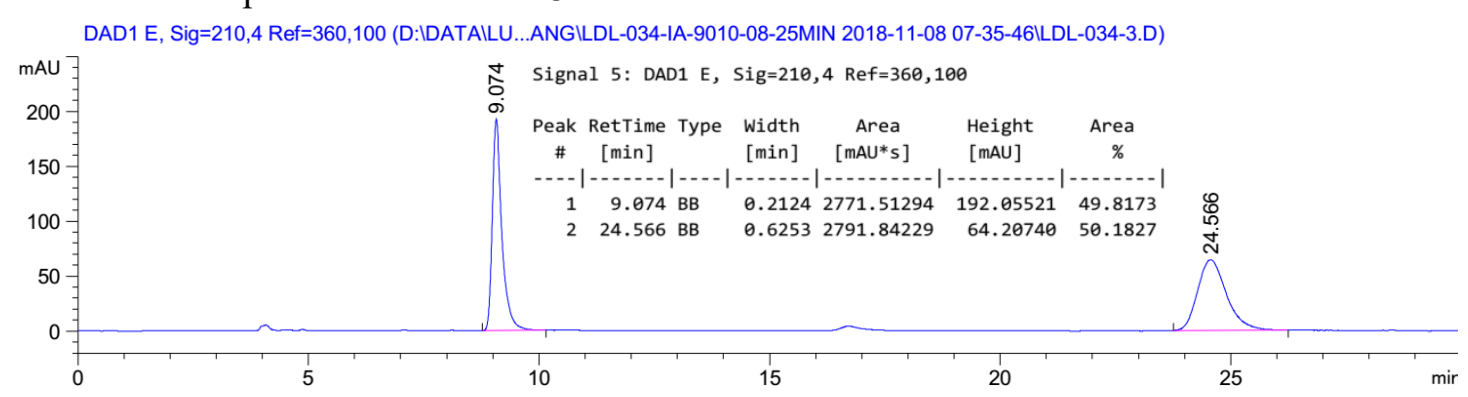

Chiral HPLC spectrum of $(R)-\mathbf{3 n}$

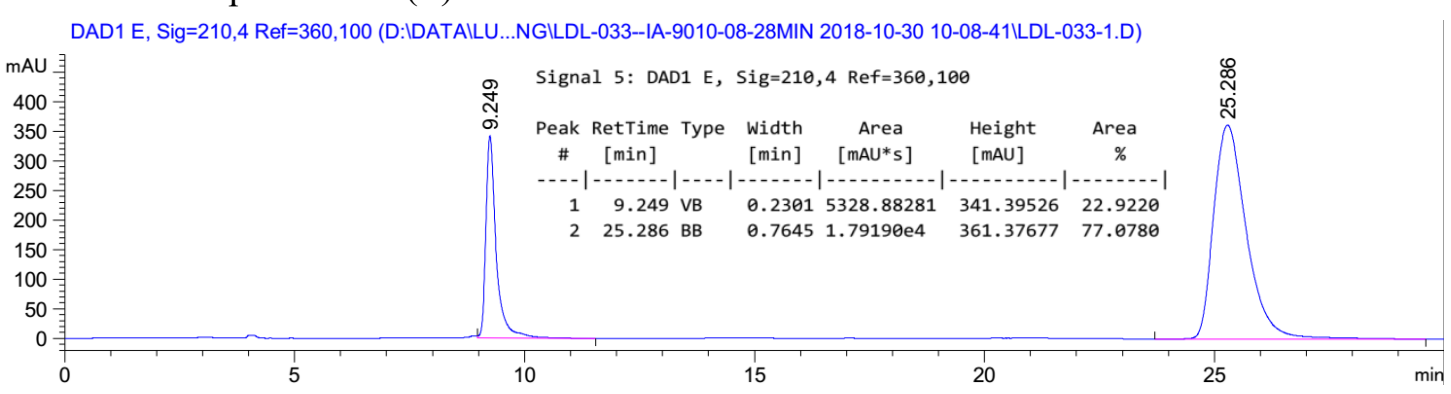




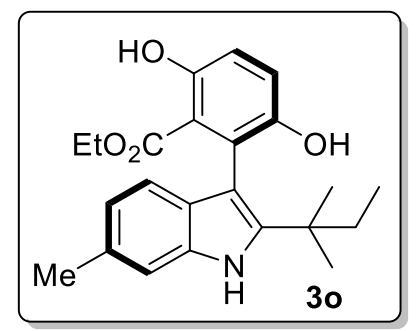

(R)-ethyl 2-(2-(tert-butyl)-5-methoxy-1 H-indol-3-yl)-3,6-dihydroxybenzoate

White solid; Yield: $63 \mathrm{mg}, 82 \%$ (petroleum ether $/ \mathrm{DCM}=2: 1)$; ee $=96 \%$; $[\alpha]_{\mathrm{D}}^{26}=-70.0(\mathrm{c}=$ $\left.0.2, \mathrm{CHCl}_{3}\right)$.

HPLC analysis: HPLC DAICEL CHIRALCEL IA, hexane/isopropanol $=90 / 10,0.8 \mathrm{~mL} / \mathrm{min}$, $\lambda=214 \mathrm{~nm}, \mathrm{t}_{\mathrm{R}}($ minor $)=7.8 \min , \mathrm{t}_{\mathrm{R}}($ major $)=24.1 \mathrm{~min}$.

${ }^{1}$ H NMR (400 MHz, CDCl$) \delta 10.85(\mathrm{~s}, 1 \mathrm{H}), 8.09(\mathrm{~s}, 1 \mathrm{H}), 7.20-7.09$ (m, 2H), 7.01 (d, J= $9.0 \mathrm{~Hz}, 1 \mathrm{H}), 6.94(\mathrm{~d}, J=8.0 \mathrm{~Hz}, 1 \mathrm{H}), 6.85(\mathrm{dd}, J=8.1,0.8 \mathrm{~Hz}, 1 \mathrm{H}), 5.00(\mathrm{~s}, 1 \mathrm{H}), 3.90(\mathrm{~m}$, 1H), $3.74(\mathrm{~m}, 1 \mathrm{H}), 2.45(\mathrm{~s}, 3 \mathrm{H}), 1.61(\mathrm{q}, J=7.5 \mathrm{~Hz}, 2 \mathrm{H}), 1.16(\mathrm{~d}, J=2.5 \mathrm{~Hz}, 6 \mathrm{H}), 0.78(\mathrm{t}, J=$ $7.4 \mathrm{~Hz}, 3 \mathrm{H}), 0.37$ (t, $J=7.2 \mathrm{~Hz}, 3 \mathrm{H})$.

${ }^{13}$ C NMR (100 MHz, CDCl $) \delta=171.04,156.23,147.79,141.87,135.39,131.96,127.71$, $121.86,121.84,121.72,118.54,118.42,113.52,110.20,105.34,60.87,37.03,36.19,27.54$, $27.02,21.84,12.62,9.39$.

HRMS (ESI) Calcd for $\mathrm{C}_{23} \mathrm{H}_{28} \mathrm{NO}_{4}{ }^{+}[\mathrm{M}+\mathrm{H}]^{+}$382.2013; Found: 382.2009 .

Chiral HPLC spectrum of racemic 30

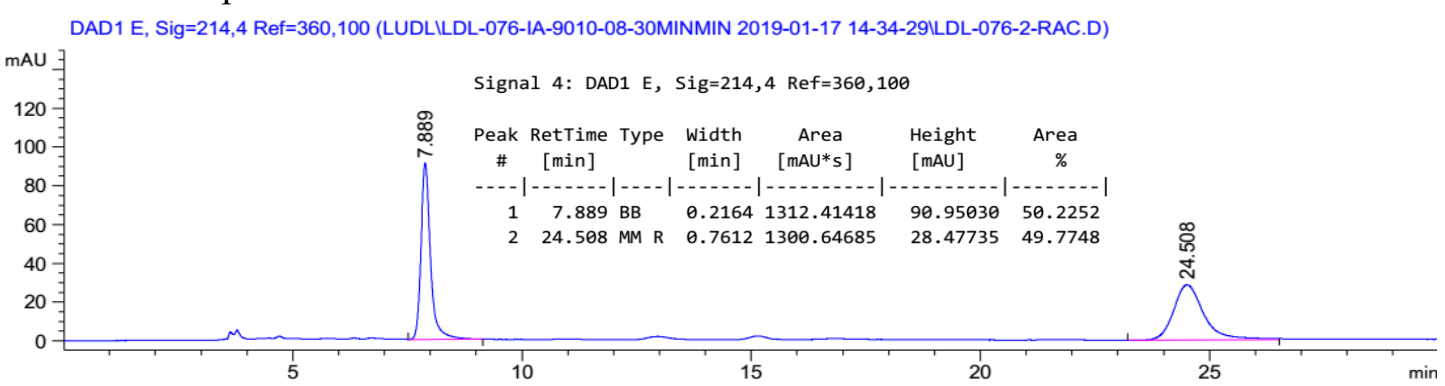

Chiral HPLC spectrum of $(R)-\mathbf{3 o}$

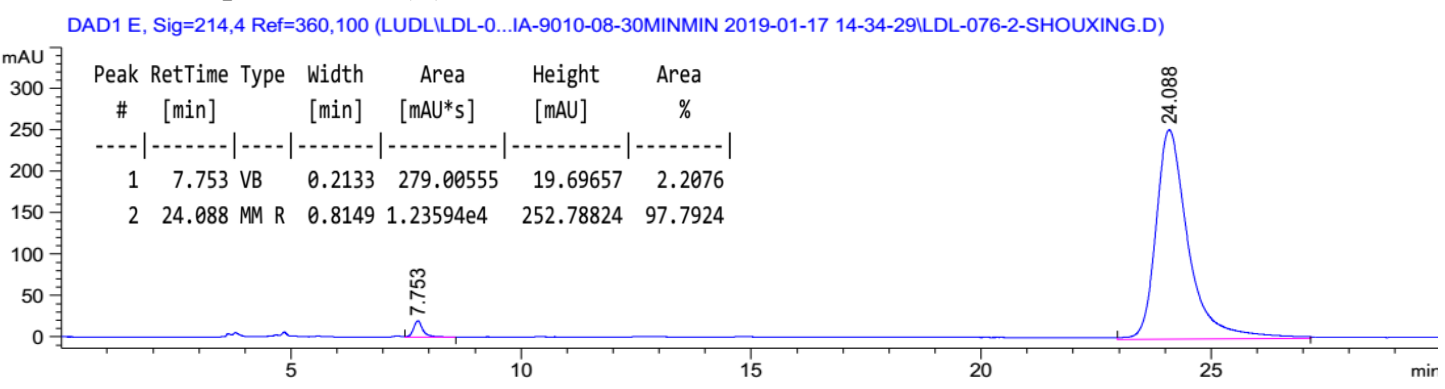




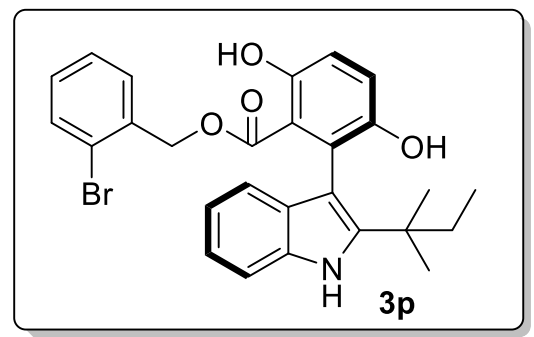

(R)-2-bromobenzyl 3,6-dihydroxy-2-(2-(tert-pentyl)-1H-indol-3-yl)benzoate

White solid; Yield: $80 \mathrm{mg}, 79 \%$ (petroleum ether/DCM $=2: 1)$; ee $=89 \%$; $[\alpha]_{\mathrm{D}}^{26}=20.0(\mathrm{c}=$ $\left.0.2, \mathrm{CHCl}_{3}\right)$.

HPLC analysis: HPLC DAICEL CHIRALCEL IA, hexane/isopropanol $=90 / 10,0.8 \mathrm{~mL} / \mathrm{min}$, $\lambda=214 \mathrm{~nm}, \mathrm{t}_{\mathrm{R}}($ minor $)=8.1 \mathrm{~min}, \mathrm{t}_{\mathrm{R}}($ major $)=13.1 \mathrm{~min}$.

${ }^{1}$ H NMR (400 MHz, CDCl 3$) \delta 10.81(\mathrm{~s}, 1 \mathrm{H}), 7.96(\mathrm{~s}, 1 \mathrm{H}), 7.34$ (d, $\left.J=8.0 \mathrm{~Hz}, 1 \mathrm{H}\right), 7.24-$ $7.11(\mathrm{~m}, 3 \mathrm{H}), 7.12-6.96(\mathrm{~m}, 4 \mathrm{H}), 6.86(\mathrm{t}, J=7.5 \mathrm{~Hz}, 1 \mathrm{H}), 6.02(\mathrm{dd}, J=7.6,1.4 \mathrm{~Hz}, 1 \mathrm{H})$, $5.07(\mathrm{~d}, J=13.1 \mathrm{~Hz}, 1 \mathrm{H}), 4.93(\mathrm{~d}, J=13.1 \mathrm{~Hz}, 1 \mathrm{H}), 4.84(\mathrm{~s}, 1 \mathrm{H}), 1.53(\mathrm{q}, J=7.4 \mathrm{~Hz}, 2 \mathrm{H})$, $1.11(\mathrm{~s}, 3 \mathrm{H}), 1.07(\mathrm{~s}, 3 \mathrm{H}), 0.71(\mathrm{t}, J=7.4 \mathrm{~Hz}, 3 \mathrm{H})$.

${ }^{13}$ C NMR (100 MHz, $\left.\mathbf{C D C l}_{3}\right) \delta=170.97,156.62,147.75,142.93,134.87,133.72,132.39$, $129.70,129.41,129.29,127.30,123.03,122.39,122.30,121.41,120.35,118.78,113.17$, $110.55,105.03,66.65,36.96,36.06,27.26,26.98,9.37$.

HRMS (ESI) Calcd for $\mathrm{C}_{27} \mathrm{H}_{27} \mathrm{BrNO}_{4}{ }^{+}[\mathrm{M}+\mathrm{H}]^{+}$508.1118; Found: 508.1109.

Chiral HPLC spectrum of racemic 3p

DAD1 C, Sig=214,4 Ref=360,100 (D:IDATAILU...061-1-4-IA-9010-08-35MIN 2018-12-18 14-41-28ILDL-061-4-RAC.D)

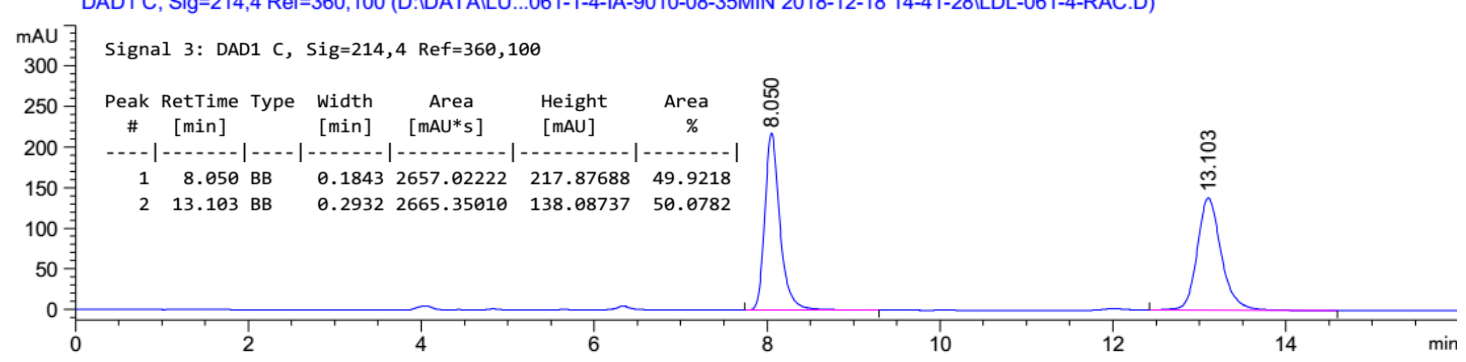

Chiral HPLC spectrum of (R)-3p DAD1 C, Sig=214,4 Ref=360,100 (D:IDATAILU...LDL-061-1-4-IA-9010-08-35MIN 2018-12-18 14-41-28ILDL-061-4.D)

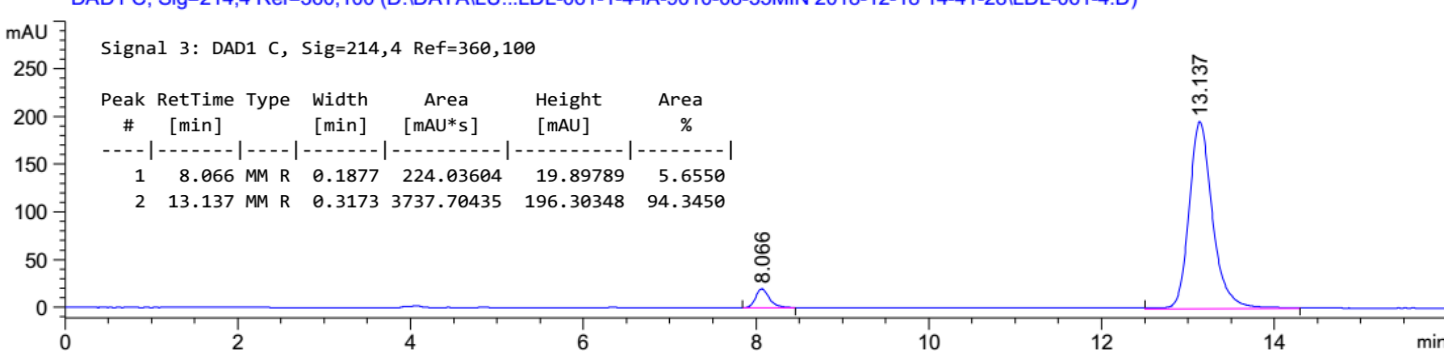




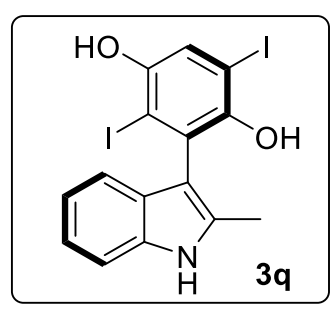

(S)-2,5-diiodo-3-(2-methyl-1 H-indol-3-yl)benzene-1,4-diol

Reaction at $25^{\circ} \mathrm{C}$ in DCE for $1.5 \mathrm{~h}$.

White solid; Yield: $42 \mathrm{mg}, 86 \%$; ee $=89 \%$; $[\alpha]_{\mathrm{D}}^{26}=7.0(\mathrm{c}=0.2$, acetone $)$.

HPLC analysis: HPLC DAICEL CHIRALCEL OD3, hexane/isopropanol $=85 / 15,0.4$ $\mathrm{mL} / \min , \lambda=230 \mathrm{~nm}, \mathrm{t}_{\mathrm{R}}($ minor $)=24.3 \mathrm{~min}, \mathrm{t}_{\mathrm{R}}($ major $)=28.1 \mathrm{~min}$.

${ }^{1}$ H NMR (400 MHz, $\left.\mathbf{C D}_{2} \mathbf{C l}_{2}\right) 8.45(\mathrm{~s}, 1 \mathrm{H}), 7.52(\mathrm{~s}, 1 \mathrm{H}), 7.46(\mathrm{~d}, \mathrm{~J}=8.1 \mathrm{~Hz}, 1 \mathrm{H}), 7.28-7.21$ (m, 1H), 7.19 (d, J = 7.5 Hz, 1H), 7.13 (t, J = $7.4 \mathrm{~Hz}, 1 \mathrm{H}), 5.36(\mathrm{~s}, 1 \mathrm{H}), 5.21(\mathrm{~s}, 1 \mathrm{H}), 2.33$ (s, $3 \mathrm{H})$.

${ }^{13} \mathbf{C}$ NMR (101 MHz, $\left.\mathbf{C D}_{2} \mathbf{C l}_{2}\right) \delta=150.12,148.85,136.11,135.29,127.20,125.52,124.45$, $122.76,120.99,119.04,111.33,111.11,95.59,82.73,12.84$.

HRMS (ESI) Calcd for $\mathrm{C}_{15} \mathrm{H}_{12} \mathrm{I}_{2} \mathrm{NO}_{2}{ }^{+}[\mathrm{M}+\mathrm{H}]^{+}$491.8952; Found: 491.8949.

Chiral HPLC spectrum of racemic 3q

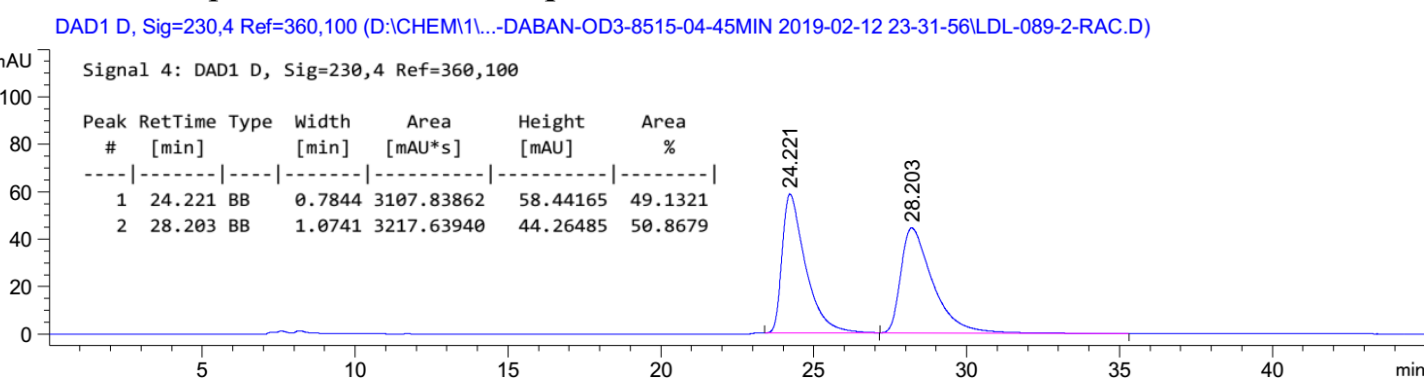

Chiral HPLC spectrum of (S)-3q

DAD1 D, Sig=230,4 Ref=360,100 (D:ICHEMI11...2-DABAN-OD3-8515-04-45MIN 2019-02-12 23-31-56ILDL-089-2-DA.D)

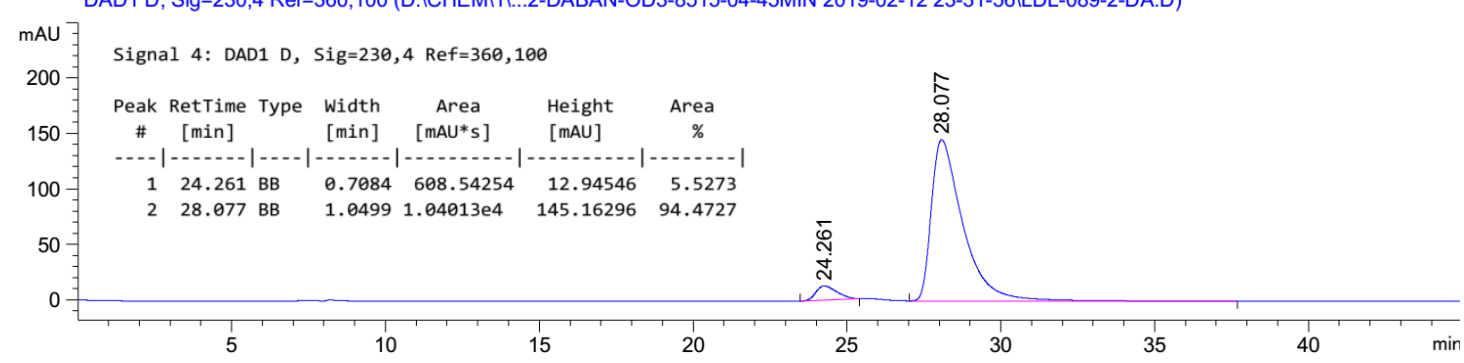




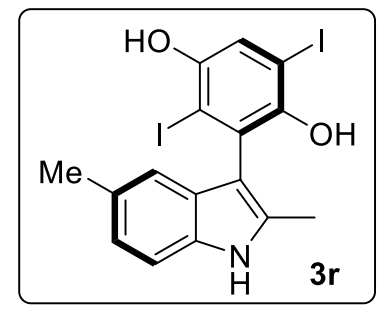

(S)-3-(2,5-dimethyl-1H-indol-3-yl)-2,5-diiodobenzene-1,4-diol

Reaction at $25{ }^{\circ} \mathrm{C}$ in DCE for $1.5 \mathrm{~h}$.

White solid; Yield: $44 \mathrm{mg}, 88 \%$; ee $=83 \% ;[\alpha]_{\mathrm{D}}^{26}=11.0(\mathrm{c}=0.2$, acetone).

HPLC analysis: HPLC DAICEL CHIRALCEL OD3, hexane/isopropanol $=88 / 12,0.3$ $\mathrm{mL} / \min , \lambda=230 \mathrm{~nm}, \mathrm{t}_{\mathrm{R}}($ minor $)=40.7 \mathrm{~min}, \mathrm{t}_{\mathrm{R}}($ major $)=45.3 \mathrm{~min}$.

${ }^{1} \mathbf{H}$ NMR (400 MHz, $\left.\mathbf{C D}_{2} \mathbf{C l}_{2}\right) \delta 8.32(\mathrm{~s}, 1 \mathrm{H}), 7.48(\mathrm{~s}, 1 \mathrm{H}), 7.30(\mathrm{~d}, \mathrm{~J}=8.3 \mathrm{~Hz}, 1 \mathrm{H}), 7.03(\mathrm{~d}, \mathrm{~J}$ $=8.3 \mathrm{~Hz}, 1 \mathrm{H}), 6.94(\mathrm{~s}, 1 \mathrm{H}), 5.36(\mathrm{~s}, 1 \mathrm{H}), 5.21(\mathrm{~s}, 1 \mathrm{H}), 2.37(\mathrm{~s}, 3 \mathrm{H}), 2.26(\mathrm{~s}, 3 \mathrm{H})$.

${ }^{13} \mathbf{C}$ NMR (100 MHz, $\left.\mathbf{C D}_{2} \mathbf{C l}_{2}\right) \delta=150.11,148.87,135.30,134.38,130.51,127.41,125.71$, $124.39,124.30,118.60,110.98,110.63,95.69,82.59,21.66,12.86$.

HRMS (ESI) Calcd for $\mathrm{C}_{16} \mathrm{H}_{14} \mathrm{I}_{2} \mathrm{NO}_{2}{ }^{+}[\mathrm{M}+\mathrm{H}]^{+}$505.9108; Found: 505.9104 .

Chiral HPLC spectrum of racemic 3r DAD1 D, Sig=230,4 Ref=360,100 (D:ICHEMI11...-090-1-OD3-8812-03-70MIN 2019-02-13 11-10-58ILDL-090-1-RAC.D)

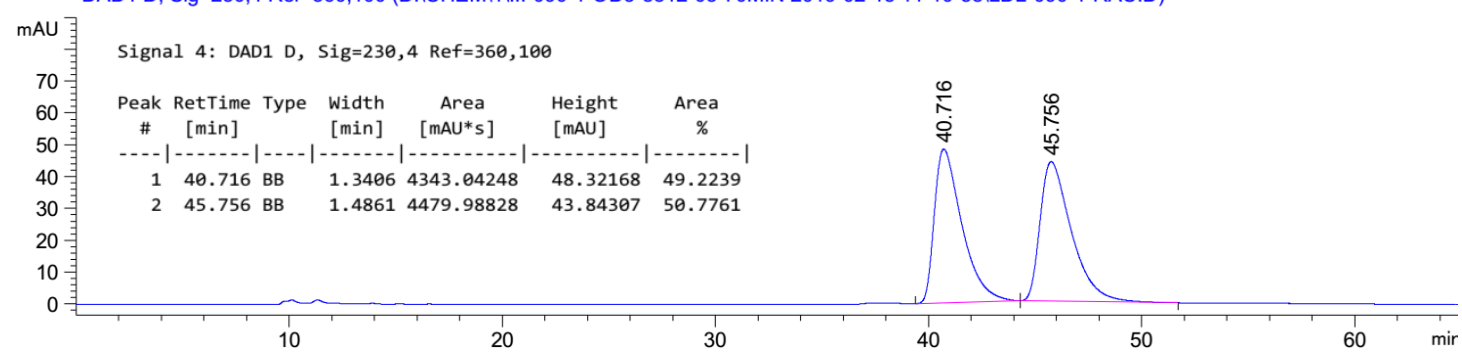

Chiral HPLC spectrum of $(S)-3 \mathbf{r}$

DAD1 D, Sig=230,4 Ref=360, 100 (D:ICHEMI11...90-1-OD3-8812-03-70MIN 2019-02-13 11-10-58LLDL-090-1-DABAN.D)
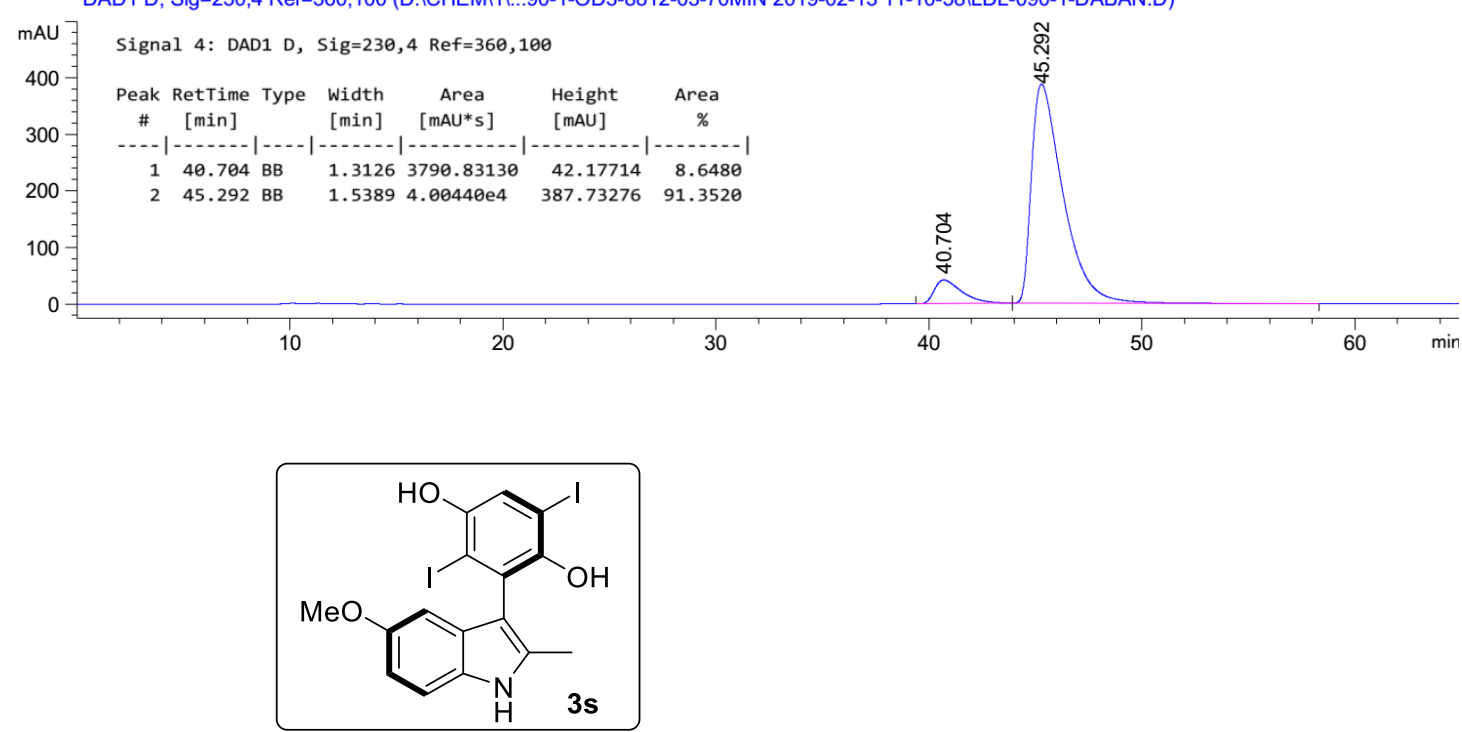

(S)-2,5-diiodo-3-(5-methoxy-2-methyl-1H-indol-3-yl)benzene-1,4-diol 
Reaction at $25^{\circ} \mathrm{C}$ in DCE for $1.5 \mathrm{~h}$.

White solid; Yield: $49 \mathrm{mg}, 95 \%$ yield; ee $=89 \% ;[\alpha]_{\mathrm{D}}^{26}=9.7(\mathrm{c}=0.2$, acetone).

HPLC analysis: HPLC DAICEL CHIRALCEL OD3, hexane/isopropanol $=85 / 15,0.5$ $\mathrm{mL} / \min , \lambda=230 \mathrm{~nm}, \mathrm{t}_{\mathrm{R}}($ minor $)=27.5 \mathrm{~min}, \mathrm{t}_{\mathrm{R}}($ major $)=33.7 \mathrm{~min}$.

${ }^{1}$ H NMR (400 MHz, CDCl $) \delta 8.15(\mathrm{~s}, 1 \mathrm{H}), 7.50(\mathrm{~s}, 1 \mathrm{H}), 7.33-7.21(\mathrm{~m}, 1 \mathrm{H}), 6.86(\mathrm{dd}, J=$ 8.8, $2.4 \mathrm{~Hz}, 1 \mathrm{H}), 6.60(\mathrm{~d}, J=2.3 \mathrm{~Hz}, 1 \mathrm{H}), 5.30(\mathrm{~s}, 1 \mathrm{H}), 5.10(\mathrm{~s}, 1 \mathrm{H}), 3.77$ (s, 3H), $2.26(\mathrm{~s}$, $3 \mathrm{H})$.

${ }^{13}$ C NMR (100 MHz, $\left.\mathbf{C D C l}_{3}\right) \delta=154.99,149.63,148.34,135.07,130.45,127.35,124.92$, $124.17,112.62,111.63,110.72,100.64,95.30,82.56,55.99,12.76$.

HRMS (ESI) Calcd for $\mathrm{C}_{16} \mathrm{H}_{14} \mathrm{I}_{2} \mathrm{NO}_{3}{ }^{+}[\mathrm{M}+\mathrm{H}]^{+}$521.9058; Found: 521.9055 .

Chiral HPLC spectrum of racemic 3s DAD1 D, Sig=230,4 Ref=360,100 (D:ICHEMI1।...-090-2-OD3-8515-05-45MIN 2019-02-13 19-43-22ILDL-090-2-RAC.D)

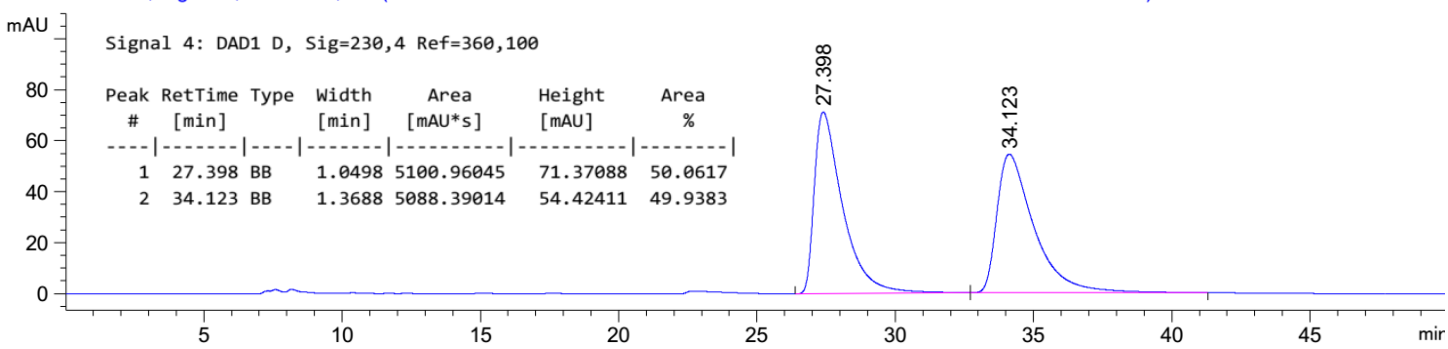

Chiral HPLC spectrum of $(S)$-3s

DAD1 D, Sig=230,4 Ref=360,100 (D:ICHEMI11...90-2-OD3-8515-05-45MIN 2019-02-13 19-43-22LLDL-090-2-DABAN.D)
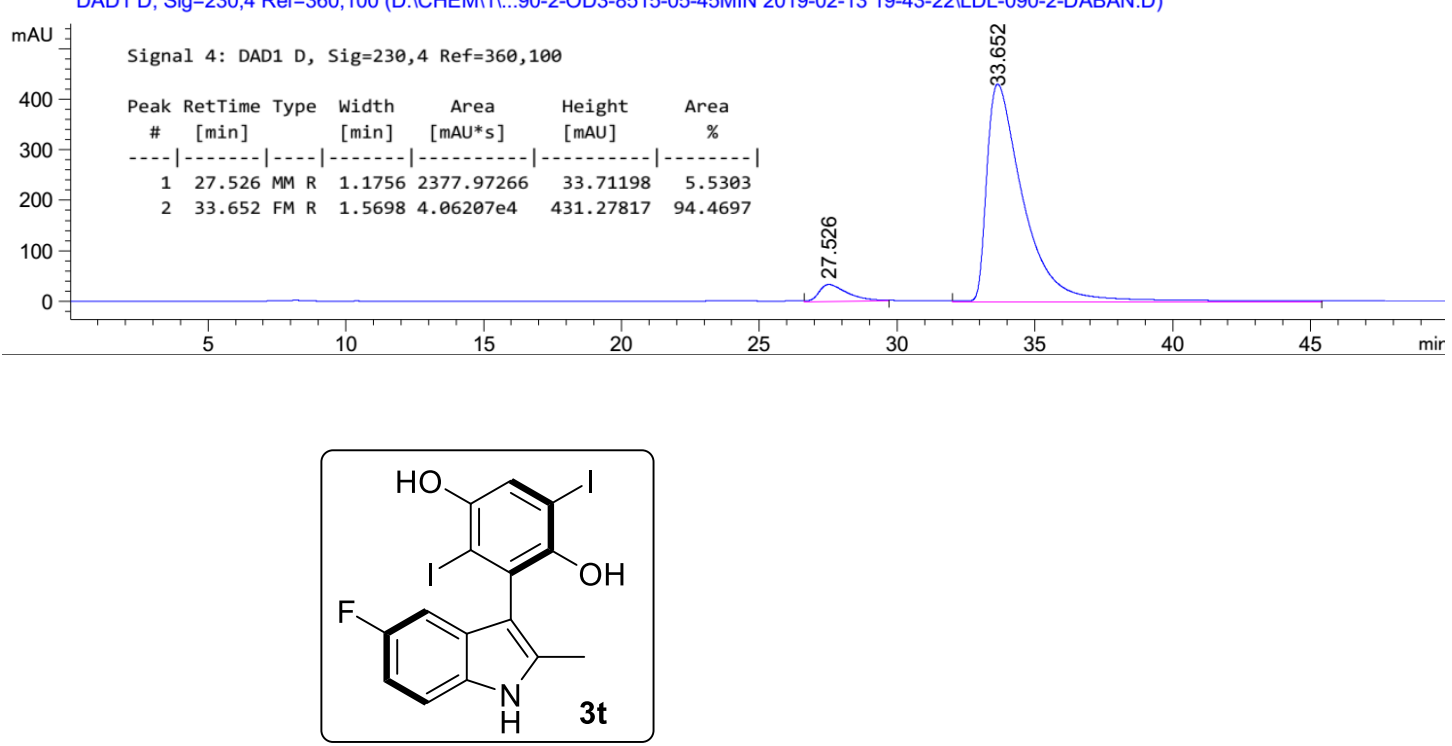

(S)-3-(5-fluoro-2-methyl-1 H-indol-3-yl)-2,5-diiodobenzene-1,4-diol

Reaction at $25{ }^{\circ} \mathrm{C}$ in $\mathrm{CCl}_{4}$ for $0.5 \mathrm{~h}$.

White solid; Yield: $46 \mathrm{mg}, 90 \%$; ee $=90 \% ;[\alpha]_{\mathrm{D}}^{26}=8.1(\mathrm{c}=0.2$, acetone) .

HPLC analysis: HPLC DAICEL CHIRALCEL OD3, hexane/isopropanol $=88 / 12,0.3$ 
$\mathrm{mL} / \min , \lambda=230 \mathrm{~nm}, \mathrm{t}_{\mathrm{R}}($ minor $)=44.9 \mathrm{~min}, \mathrm{t}_{\mathrm{R}}($ major $)=49.9 \mathrm{~min}$.

${ }^{1}$ H NMR (400 MHz, $\left.\mathbf{C D}_{2} \mathbf{C l}_{2}\right) \delta 8.59(\mathrm{~s}, 1 \mathrm{H}), 7.47(\mathrm{~s}, 1 \mathrm{H}), 7.34(\mathrm{dd}, J=8.8,4.3 \mathrm{~Hz}, 1 \mathrm{H}), 6.94$ $(\mathrm{td}, J=9.1,2.4 \mathrm{~Hz}, 1 \mathrm{H}), 6.81(\mathrm{dd}, J=9.4,2.3 \mathrm{~Hz}, 1 \mathrm{H}), 5.49(\mathrm{~s}, 1 \mathrm{H}), 5.32(\mathrm{~d}, J=3.7 \mathrm{~Hz}, 1 \mathrm{H})$, $2.27(\mathrm{~s}, 3 \mathrm{H})$.

${ }^{13} \mathbf{C}$ NMR (100 MHz, $\left.\mathbf{C D}_{2} \mathbf{C l}_{2}\right) \delta=159.98,157.64,150.29,148.78,137.33,132.55,127.97$, $127.87,125.11,124.64,112.14,112.04,111.51,111.46,110.79,110.53,104.25,104.01$, $95.43,82.95,12.92$.

${ }^{19}$ F NMR (376 MHz, $\left.\mathrm{CD}_{2} \mathrm{Cl}_{2}\right) \delta=-124.02$.

HRMS (ESI) Calcd for $\mathrm{C}_{15} \mathrm{H}_{11} \mathrm{FI}_{2} \mathrm{NO}_{2}{ }^{+}[\mathrm{M}+\mathrm{H}]^{+}$509.8858; Found: 509.8859.

Chiral HPLC spectrum of racemic 3t DAD1 D, Sig=230,4 Ref=360,100 (D:ICHEMI1I...D38812-03-70MIN-20190218 2019-02-18 21-31-43ILDL-092-2-RAC.D)

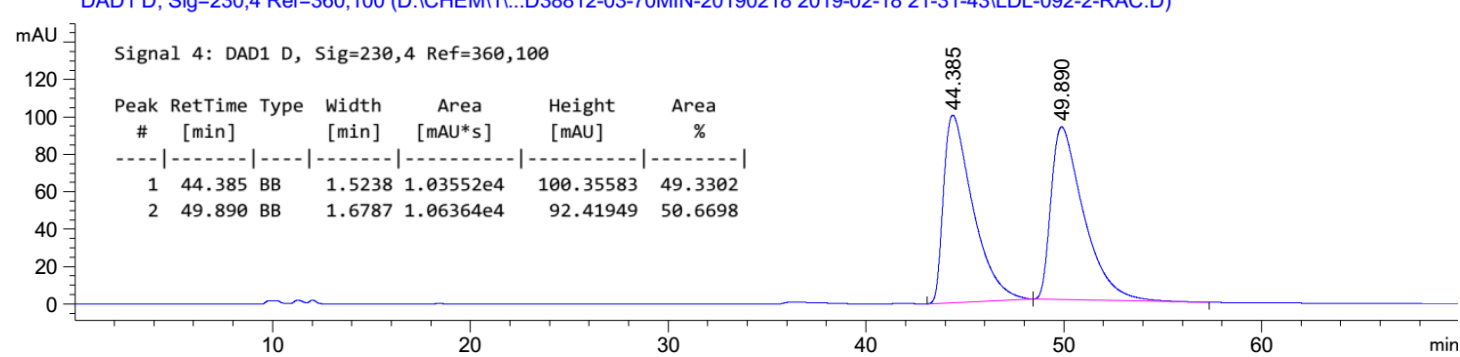

Chiral HPLC spectrum of $(S)-3 \mathbf{t}$

DAD1 D, Sig=230,4 Ref=360,100 (D:ICHEMI11...38812-03-70MIN-20190218 2019-02-18 21-31-43ILDL-092-2-GUO-.D)
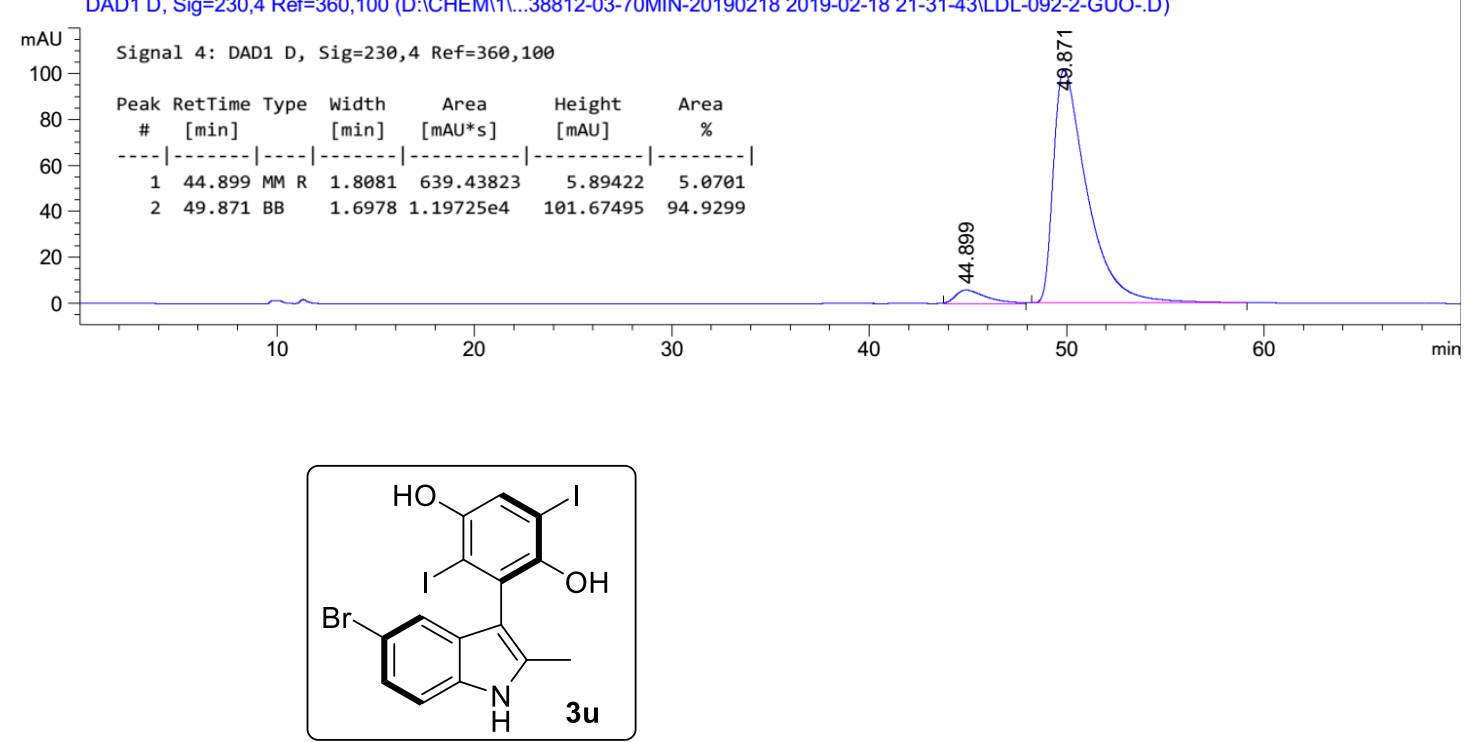

(S)-3-(5-bromo-2-methyl-1 H-indol-3-yl)-2,5-diiodobenzene-1,4-diol

Reaction at $25{ }^{\circ} \mathrm{C}$ in $\mathrm{CCl}_{4}$ for $0.5 \mathrm{~h}$.

White solid; Yield: $49 \mathrm{mg}, 85 \%$; ee $=93 \% ;[\alpha]_{\mathrm{D}}^{26}=15.5(\mathrm{c}=0.2$, acetone $)$.

HPLC analysis: HPLC DAICEL CHIRALCEL OD3, hexane/isopropanol $=85 / 15,0.4$ $\mathrm{mL} / \min , \lambda=230 \mathrm{~nm}, \mathrm{t}_{\mathrm{R}}($ minor $)=24.5 \mathrm{~min}, \mathrm{t}_{\mathrm{R}}($ major $)=28.2 \mathrm{~min}$.

${ }^{1}$ H NMR (400 MHz, $\left.\mathbf{C D}_{2} \mathbf{C l}_{2}\right) \delta 8.50(\mathrm{~s}, 1 \mathrm{H}), 7.48(\mathrm{~s}, 1 \mathrm{H}), 7.36-7.16(\mathrm{~m}, 3 \mathrm{H}), 5.25(\mathrm{~d}, J=$ 
$2.3 \mathrm{~Hz}, 2 \mathrm{H}), 2.27$ (s, 3H).

${ }^{13}$ C NMR (100 MHz, $\left.\mathbf{C D}_{2} \mathbf{C l}_{2}\right) \delta=150.22,148.79,136.82,134.70,129.11,125.43,124.76$, $124.72,121.54,114.11,112.89,111.00,95.51,83.16,12.85$.

HRMS (ESI) Calcd for $\mathrm{C}_{15} \mathrm{H}_{11} \mathrm{BrI}_{2} \mathrm{NO}_{2}{ }^{+}[\mathrm{M}+\mathrm{H}]^{+}$569.8057; Found: 569.8059 .

Chiral HPLC spectrum of racemic $3 \mathbf{u}$

DAD1 D, Sig=230,4 Ref=360,100 (D:ICHEMI1।..D38515-04-45MIN-20190218 2019-02-18 11-09-51LLL-089-1-RAC.D)

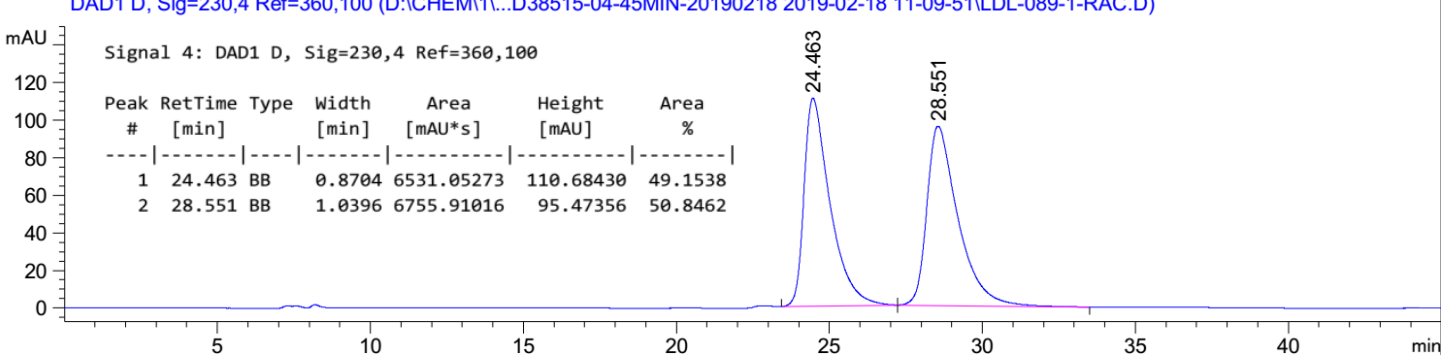

Chiral HPLC spectrum of $(S)-\mathbf{3 u}$

DAD1 D, Sig=230,4 Ref=360,100 (D:ICHEMI1I...38515-04-45MIN-20190218 2019-02-18 11-09-51|LDL-089-1-CCL4.D)
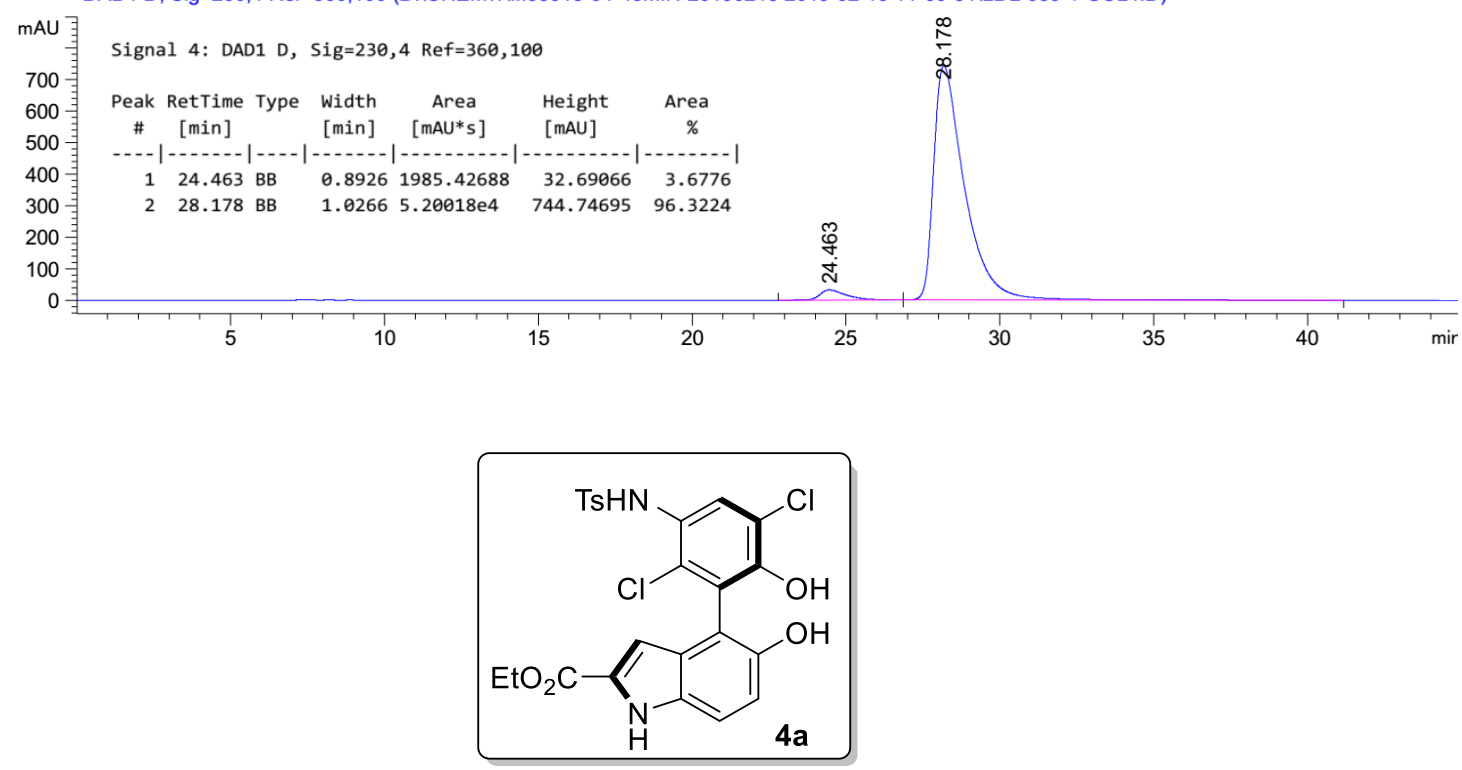

ethyl (S)-4-(2,5-dichloro-6-hydroxy-3-((4-methylphenyl)sulfonamido)phenyl)-5-hydroxy-

$1 \mathrm{H}$-indole-2-carboxylate

White solid; Yield: $99 \mathrm{mg}, 93 \%$ (petroleum ether/ethyl acetate $=3: 2$ ); ee $=90 \%$; $[\alpha]_{\mathrm{D}}^{26}=30.7$ $(\mathrm{c}=0.2, \mathrm{MeOH})$.

HPLC analysis: HPLC DAICEL CHIRALCEL IG, hexane/isopropanol $(0.1 \%$ TFA $)=60 / 40$, $1.0 \mathrm{~mL} / \mathrm{min}, \lambda=214 \mathrm{~nm}, \mathrm{t}_{\mathrm{R}}($ minor $)=12.7 \mathrm{~min}, \mathrm{t}_{\mathrm{R}}($ major $)=27.3 \mathrm{~min}$.

${ }^{1}$ H NMR (400 MHz, Acetone-d $\left.{ }_{6}\right) \delta 10.80$ (s, 1H), 8.40 (s, 1H), 8.19 (s, 1H), $7.94(\mathrm{~s}, 1 \mathrm{H})$, $7.79-7.59$ (m, 3H), $7.43(\mathrm{~d}, J=8.9 \mathrm{~Hz}, 1 \mathrm{H}), 7.37(\mathrm{~d}, J=8.2 \mathrm{~Hz}, 2 \mathrm{H}), 7.01(\mathrm{~d}, J=8.9 \mathrm{~Hz}$, 1H), $6.48(\mathrm{~d}, J=1.5 \mathrm{~Hz}, 1 \mathrm{H}), 4.54-4.22(\mathrm{~m}, 2 \mathrm{H}), 2.33(\mathrm{~s}, 3 \mathrm{H}), 1.37$ (t, $J=7.1 \mathrm{~Hz}, 3 \mathrm{H})$.

${ }^{13}$ C NMR (100 MHz, Acetone-d $\left.{ }_{6}\right) \delta=162.10,151.43,149.94,144.53,138.01,133.17$, $130.62,130.38,128.90,128.49,128.32,127.93,127.35,125.28,120.01,117.42,114.74$, 
$111.49,107.01,61.26,21.38,14.70$.

HRMS (ESI) Calcd for $\mathrm{C}_{24} \mathrm{H}_{21} \mathrm{Cl}_{2} \mathrm{~N}_{2} \mathrm{O}_{6} \mathrm{~S}^{+}[\mathrm{M}+\mathrm{H}]^{+}$535.0492; Found: 535.0491 .

Chiral HPLC spectrum of racemic $\mathbf{4 a}$

DAD1 C, Sig=214,4 Ref=360,100 (D:IDATAILU...ILDL-134-IG-6040-10-60MIN 2019-05-02 22-20-50ILDL-134-5RAC.D)

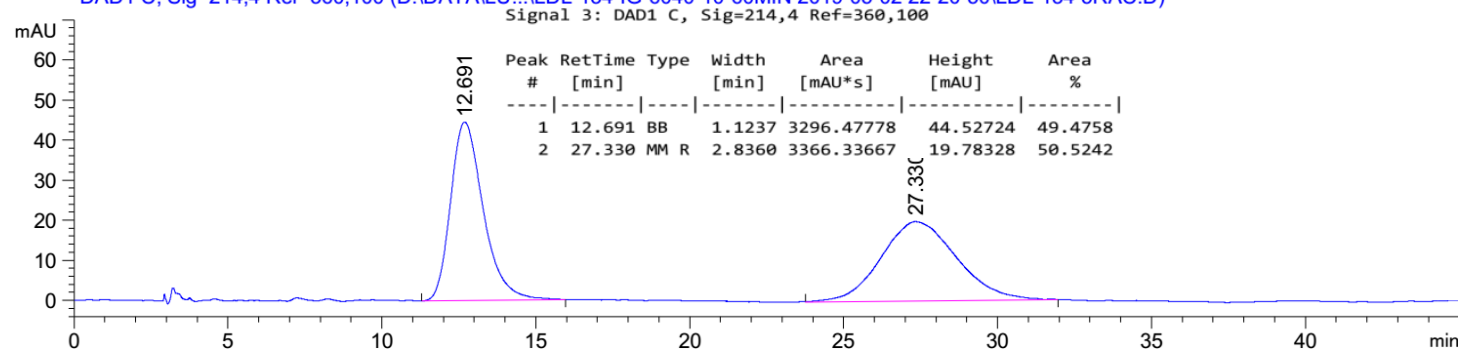

Chiral HPLC spectrum of $(S)-\mathbf{4 a}$

DAD1 C, Sig=214,4 Ref=360,100 (D:IDATAILU...ANGILDL-134-IG-6040-10-60MIN 2019-05-02 22-20-50ILDL-134-5.D)
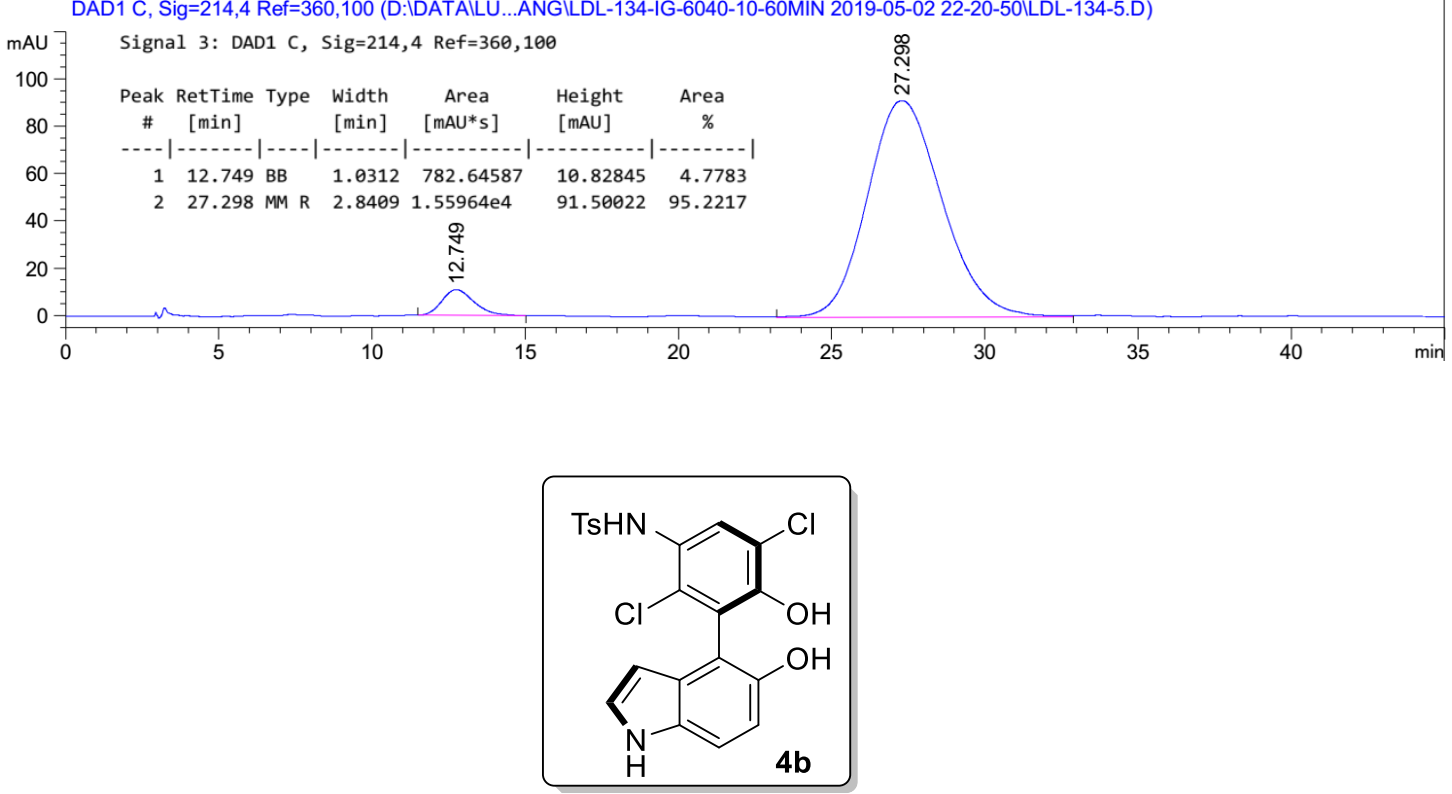

(S)-N-(2,5-dichloro-4-hydroxy-3-(5-hydroxy-1 H-indol-4-yl)phenyl)-4-methylbenzenesulfonamide

Pale yellow solid; Yield: $54 \mathrm{mg}, 58 \%$ (petroleum ether/ethyl acetate $=3: 2$ ); ee $=95 \%$; $[\alpha]_{\mathrm{D}}^{26}=$ $23.7(\mathrm{c}=0.2, \mathrm{MeOH})$.

HPLC analysis: HPLC DAICEL CHIRALCEL IA, hexane/isopropanol $=60 / 40,1.0 \mathrm{~mL} / \mathrm{min}$, $\lambda=254 \mathrm{~nm}, \mathrm{t}_{\mathrm{R}}($ major $)=13.3 \mathrm{~min}, \mathrm{t}_{\mathrm{R}}($ minor $)=33.1 \mathrm{~min}$.

${ }^{1}$ H NMR (400 MHz, Acetone-d $\left.{ }_{6}\right) \delta 10.07(\mathrm{~s}, 1 \mathrm{H}), 8.36(\mathrm{~s}, 1 \mathrm{H}), 8.05-7.72(\mathrm{~m}, 2 \mathrm{H}), 7.66(\mathrm{dd}$, $J=7.5,5.9 \mathrm{~Hz}, 3 \mathrm{H}), 7.34(\mathrm{~d}, J=8.0 \mathrm{~Hz}, 2 \mathrm{H}), 7.30(\mathrm{dd}, J=8.7,0.8 \mathrm{~Hz}, 1 \mathrm{H}), 7.22(\mathrm{t}, J=2.7$

$\mathrm{Hz}, 1 \mathrm{H}), 6.81(\mathrm{~d}, J=8.7 \mathrm{~Hz}, 1 \mathrm{H}), 5.71-5.57(\mathrm{~m}, 1 \mathrm{H}), 2.39(\mathrm{~s}, 3 \mathrm{H})$.

${ }^{13}$ C NMR (100 MHz, Acetone-d $\left.{ }_{6}\right) \delta=151.20,148.97,144.52,137.87,131.67,130.34$, $129.09,128.08,127.55,127.17,126.23,119.73,113.52,112.65,110.35,101.14,21.47$.

HRMS (ESI) Calcd for $\mathrm{C}_{21} \mathrm{H}_{17} \mathrm{Cl}_{2} \mathrm{~N}_{2} \mathrm{O}_{4} \mathrm{~S}^{+}[\mathrm{M}+\mathrm{H}]^{+} 463.0281$; Found: 463.0277 . 
Chiral HPLC spectrum of racemic $\mathbf{4 b}$

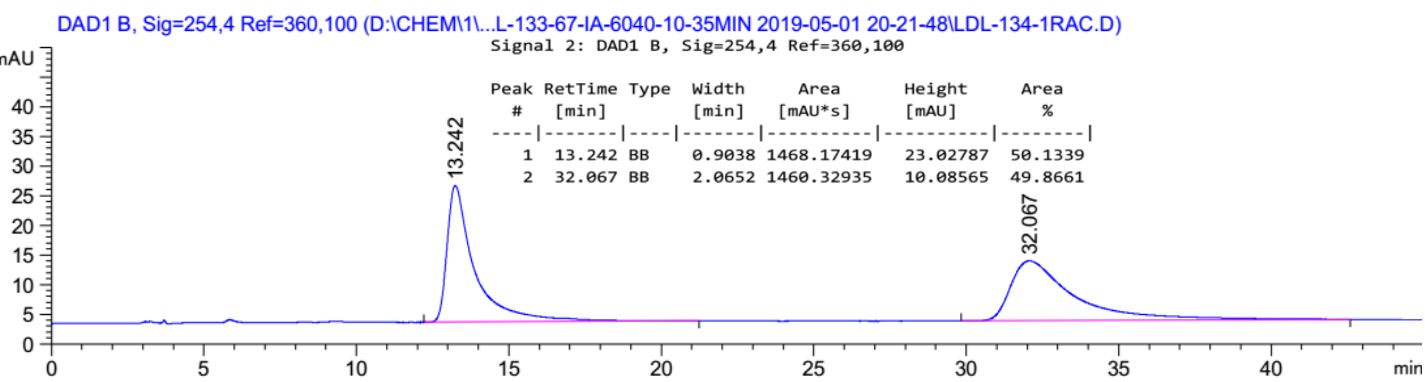

Chiral HPLC spectrum of $(S)-\mathbf{4 b}$

DAD1 B, Sig=254,4 Ref=360,100 (D:ICHEMI1I...ILDL-133-67-IA-6040-10-35MIN 2019-05-01 20-21-48ILDL-133-7.D)
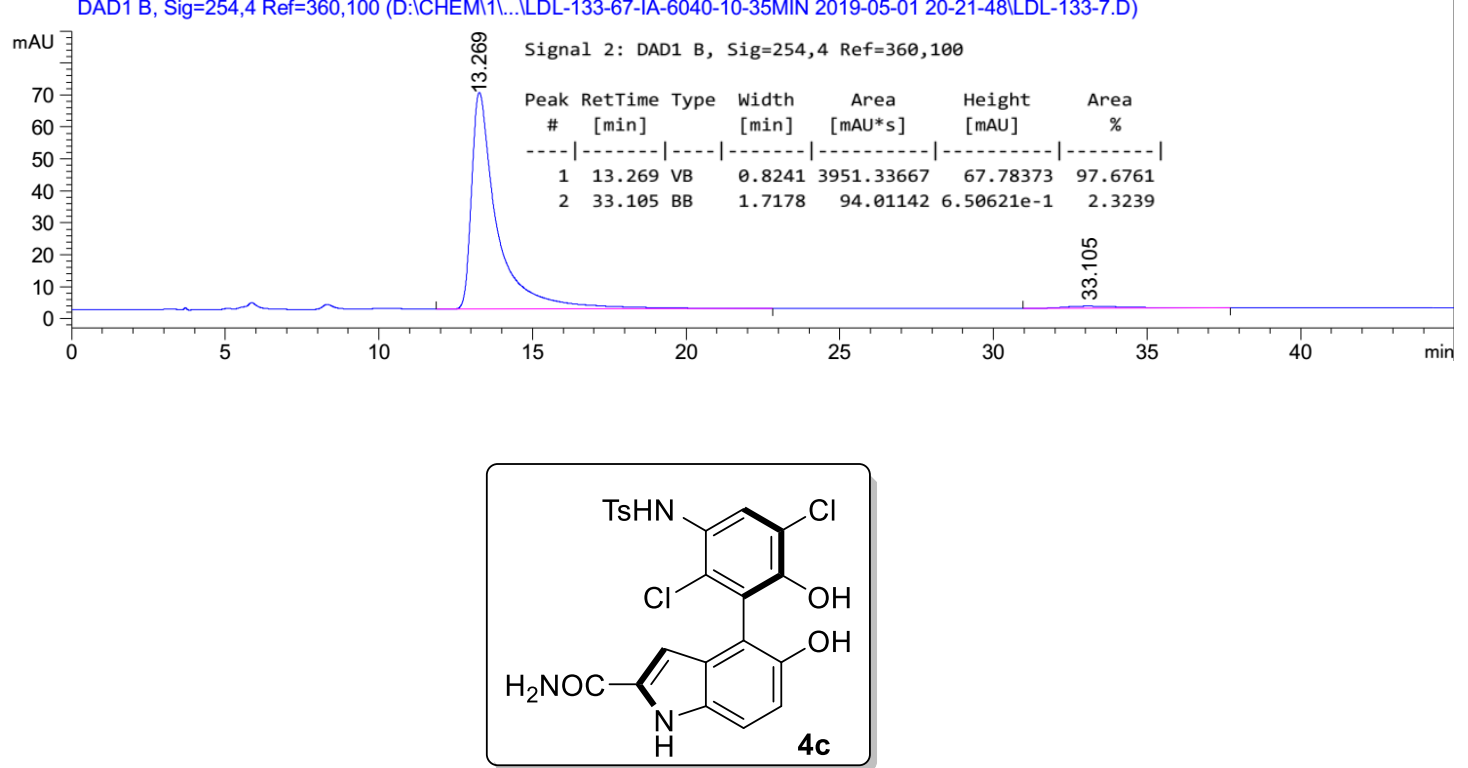

(S)-4-(2,5-dichloro-6-hydroxy-3-((4-methylphenyl)sulfonamido)phenyl)-5-hydroxy-1 H-indole-2carboxamide

Pale yellow solid; Yield: $81 \mathrm{mg}, 80 \%$ (petroleum ether/ethyl acetate $=2: 3$ ); ee $=95 \%$; $[\alpha]_{\mathrm{D}}^{26}=$ $29.0(\mathrm{c}=0.2, \mathrm{MeOH})$.

HPLC analysis: HPLC DAICEL CHIRALCEL ID, hexane/isopropanol $(0.1 \%$ TFA $)=40 / 60$, $1.0 \mathrm{~mL} / \mathrm{min}, \lambda=254 \mathrm{~nm}, \mathrm{t}_{\mathrm{R}}($ minor $)=6.3 \mathrm{~min}, \mathrm{t}_{\mathrm{R}}$ (major) $=12.2 \mathrm{~min}$.

${ }^{1}$ H NMR (400 MHz, Acetone-d $\left.{ }_{6}\right) \delta 10.71(\mathrm{~s}, 1 \mathrm{H}), 8.42(\mathrm{~s}, 1 \mathrm{H}), 8.16(\mathrm{~s}, 1 \mathrm{H}), 7.83(\mathrm{~s}, 1 \mathrm{H})$, $7.70-7.54$ (m, 4H), 7.41 (d, $J=8.8 \mathrm{~Hz}, 1 \mathrm{H}), 7.36(\mathrm{~d}, J=8.0 \mathrm{~Hz}, 2 \mathrm{H}), 6.92(\mathrm{~d}, J=8.8 \mathrm{~Hz}$, 1H), $6.80(\mathrm{~s}, 1 \mathrm{H}), 6.45$ (d, $J=1.5 \mathrm{~Hz}, 1 \mathrm{H}), 2.33(\mathrm{~s}, 3 \mathrm{H})$.

${ }^{13}$ C NMR (100 MHz, Acetone-d $) \delta=163.96,151.45,149.84,144.49,138.35,132.75$, $132.55,130.72,130.44,129.06,128.21,128.00,127.43,125.67,119.82,116.10,114.62$, $111.26,102.44,21.45$.

HRMS (ESI) Calcd for $\mathrm{C}_{22} \mathrm{H}_{18} \mathrm{Cl}_{2} \mathrm{~N}_{3} \mathrm{O}_{5} \mathrm{~S}^{+}[\mathrm{M}+\mathrm{H}]^{+}$506.0339; Found: 506.0340.

Chiral HPLC spectrum of racemic $\mathbf{4 c}$ 


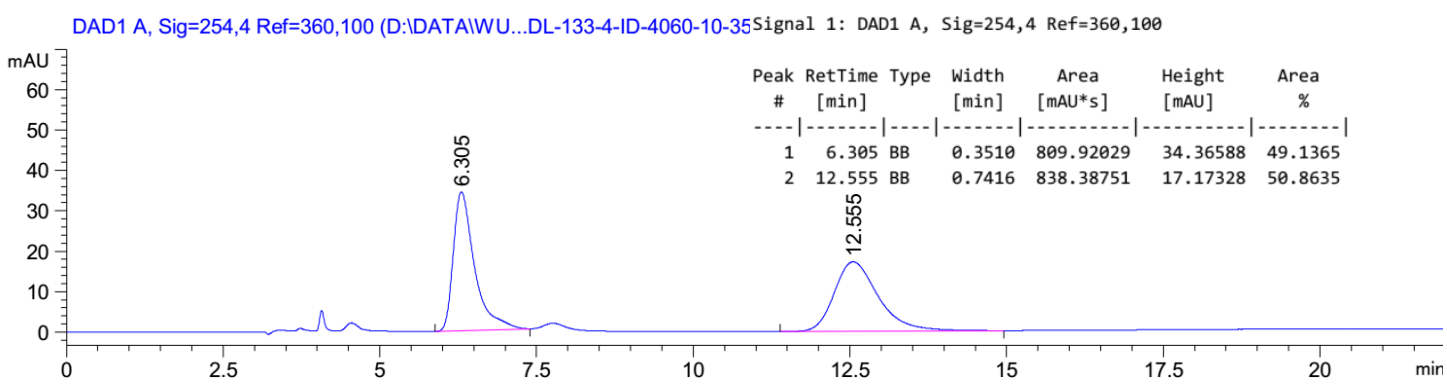

Chiral HPLC spectrum of $(S)-\mathbf{4 c}$

DAD1 A, Sig=254,4 Ref=360,100 (D:IDATAIWU....LDL-133-4-ID-4060-10-35MIN 2019-05-03 21-33-17ILDL-133-4-.D)
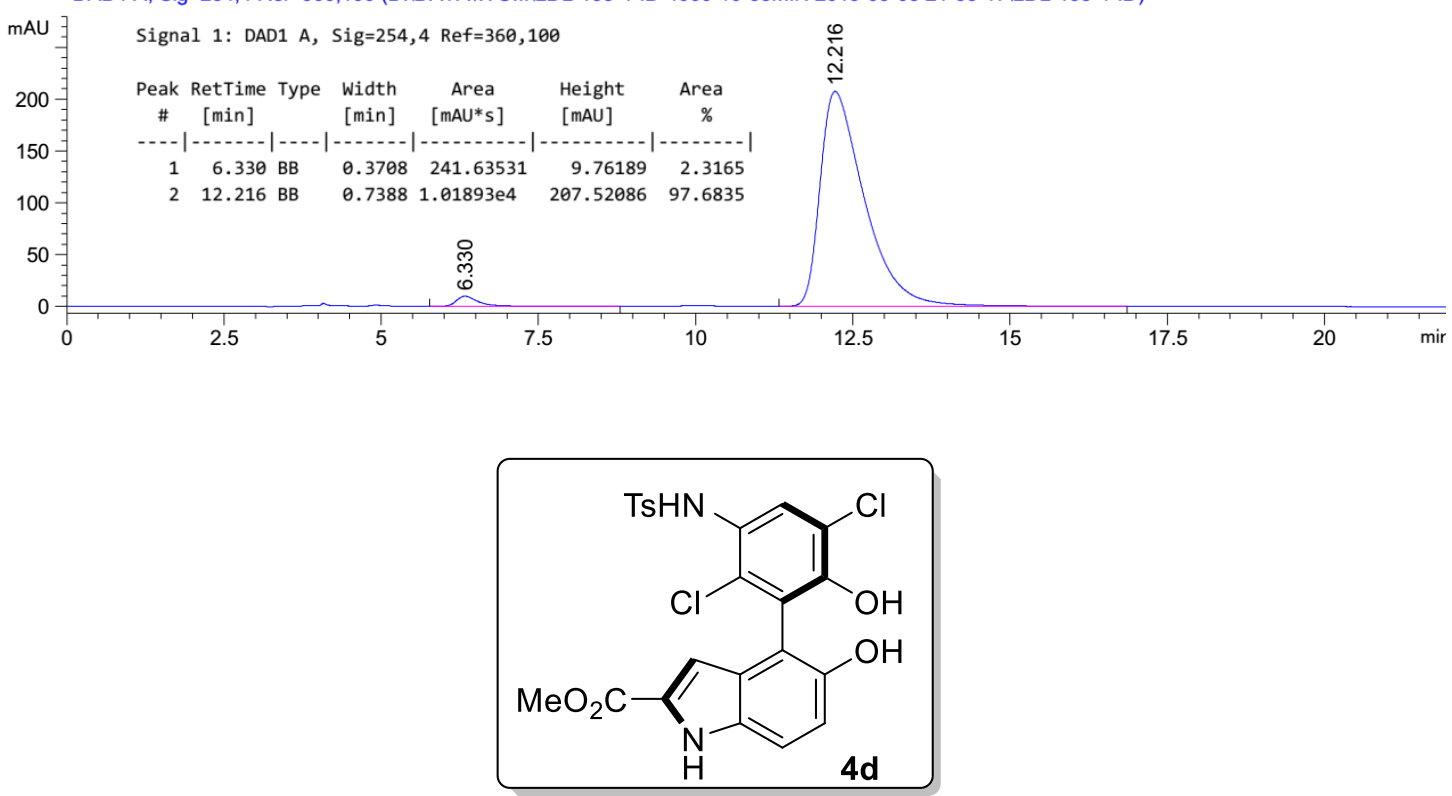

methyl (S)-4-(2,5-dichloro-6-hydroxy-3-((4-methylphenyl)sulfonamido)phenyl)-5-hydroxy$1 H$-indole-2-carboxylate

Pale yellow solid; Yield: $99 \mathrm{mg}, 95 \%$ (petroleum ether/ethyl acetate $=3: 2$ ); ee $=93 \%$; $[\alpha]_{\mathrm{D}}^{26}=$ $39.6(\mathrm{c}=0.2, \mathrm{MeOH})$.

HPLC analysis: HPLC DAICEL CHIRALCEL IG, hexane/isopropanol $(0.1 \%$ TFA $)=60 / 40$, $0.8 \mathrm{~mL} / \mathrm{min}, \lambda=214 \mathrm{~nm}, \mathrm{t}_{\mathrm{R}}($ minor $)=15.9 \mathrm{~min}, \mathrm{t}_{\mathrm{R}}($ major $)=27.5 \mathrm{~min}$.

${ }^{1}$ H NMR (400 MHz, Acetone-d $\left.{ }_{6}\right) \delta 10.83(\mathrm{~s}, 1 \mathrm{H}), 8.41(\mathrm{~s}, 1 \mathrm{H}), 8.21(\mathrm{~s}, 1 \mathrm{H}), 7.98(\mathrm{~s}, 1 \mathrm{H})$, $7.65(\mathrm{~d}, J=8.5 \mathrm{~Hz}, 3 \mathrm{H}), 7.42(\mathrm{dd}, J=8.9,0.6 \mathrm{~Hz}, 1 \mathrm{H}), 7.37(\mathrm{~d}, J=8.1 \mathrm{~Hz}, 2 \mathrm{H}), 7.01(\mathrm{~d}, J=$ $8.9 \mathrm{~Hz}, 1 \mathrm{H}), 6.44$ (d, $J=1.4 \mathrm{~Hz}, 1 \mathrm{H}), 3.90$ (s, 3H), 2.33 (s, 3H).

${ }^{13}$ C NMR (100 MHz, Acetone-d $\left.\mathbf{d}_{6}\right) \delta=162.55,151.45,149.98,144.57,138.03,133.24$, $130.57,130.40,128.56,128.50,128.30,127.96,127.36,125.30,120.03,117.52,114.76$, $111.56,107.17,52.03,21.37$.

HRMS (ESI) Calcd for $\mathrm{C}_{23} \mathrm{H}_{19} \mathrm{Cl}_{2} \mathrm{~N}_{2} \mathrm{O}_{6} \mathrm{~S}^{+}[\mathrm{M}+\mathrm{H}]^{+}$521.0335; Found: 521.0335 .

Chiral HPLC spectrum of racemic $\mathbf{4 d}$ 


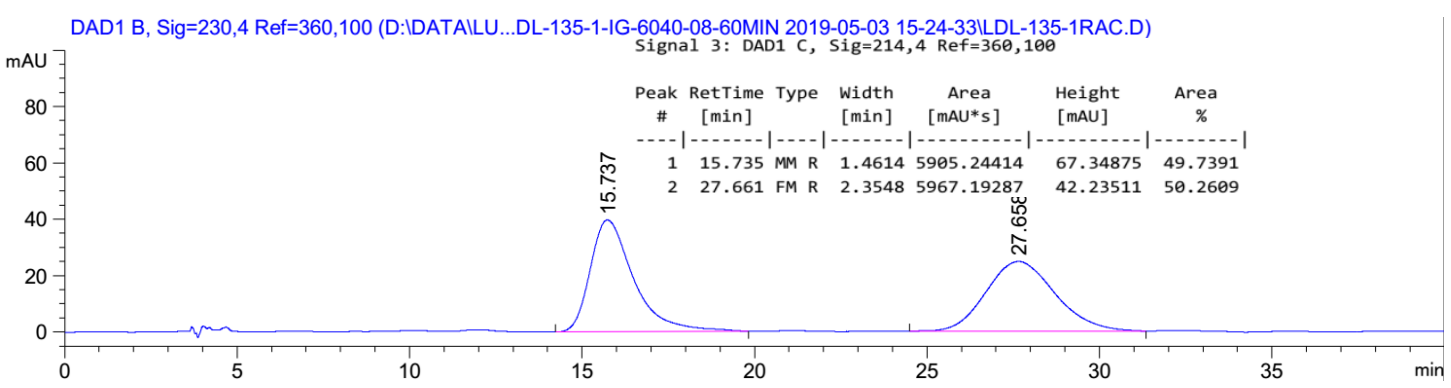

Chiral HPLC spectrum of $(S)-\mathbf{4 d}$

DAD1 C, Sig=214,4 Ref=360,100 (D:IDATAILU...GILDL-135-1-IG-6040-08-60MIN 2019-05-03 15-24-33ILDL-135-1.D)
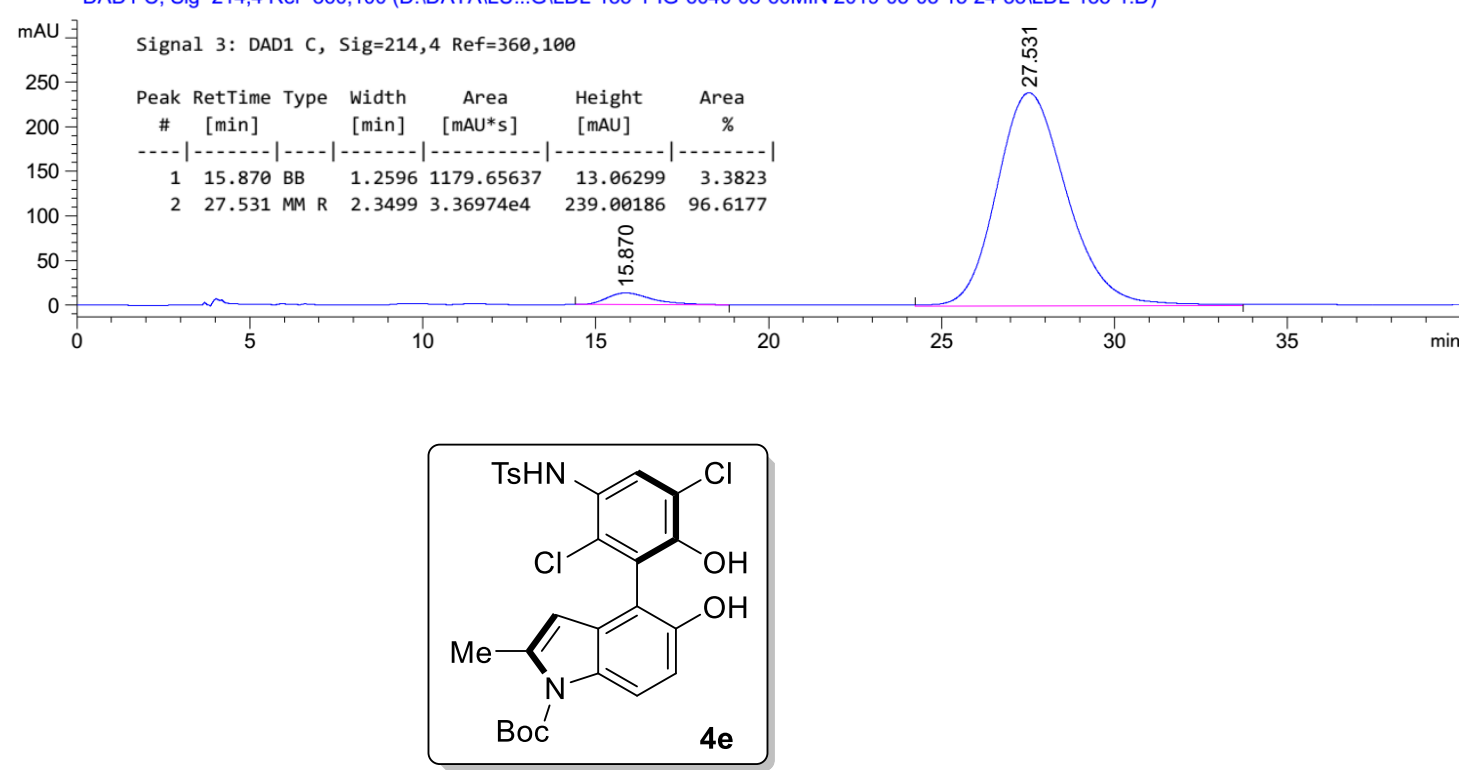

tert-butyl (S)-4-(2,5-dichloro-6-hydroxy-3-((4-methylphenyl)sulfonamido)phenyl)-5hydroxy-2-methyl-1 H-indole-1-carboxylate

White solid; Yield: $109 \mathrm{mg}, 95 \%$ (petroleum ether/ethyl acetate $=4: 1$ ); ee $=92 \% ;[\alpha]_{\mathrm{D}}^{26}=$ $19.6(\mathrm{c}=0.2, \mathrm{MeOH})$.

HPLC analysis: HPLC DAICEL CHIRALCEL ADH, hexane/isopropanol $=70 / 30,0.8$ $\mathrm{mL} / \mathrm{min}, \lambda=230 \mathrm{~nm}, \mathrm{t}_{\mathrm{R}}($ major $)=12.5 \mathrm{~min}, \mathrm{t}_{\mathrm{R}}($ minor $)=23.1 \mathrm{~min}$.

${ }^{1} \mathbf{H}$ NMR (400 MHz, CDCl $) \delta 8.04(\mathrm{~d}, \mathrm{~J}=9.0 \mathrm{~Hz}, 1 \mathrm{H}), 7.80(\mathrm{~s}, 1 \mathrm{H}), 7.63(\mathrm{~d}, \mathrm{~J}=8.3 \mathrm{~Hz}, 2 \mathrm{H})$, $7.24(\mathrm{~d}, \mathrm{~J}=8.1 \mathrm{~Hz}, 2 \mathrm{H}), 6.84(\mathrm{~d}, \mathrm{~J}=9.0 \mathrm{~Hz}, 1 \mathrm{H}), 6.80(\mathrm{~s}, 1 \mathrm{H}), 5.53(\mathrm{~s}, 2 \mathrm{H}), 4.77$ (s, 1H), 2.51 $(\mathrm{d}, \mathrm{J}=0.7 \mathrm{~Hz}, 3 \mathrm{H}), 2.40(\mathrm{~s}, 3 \mathrm{H}), 1.66(\mathrm{~s}, 9 \mathrm{H})$.

${ }^{13}$ C NMR (100 MHz, $\left.\mathbf{C D C l}_{3}\right) \delta=150.39,148.98,148.81,144.47,139.62,135.62,131.13$, $129.72,129.34,127.55,127.49,127.00,125.77,122.28,119.95,117.77,112.43,108.91$, $106.24,84.15,28.32,21.72,17.33$.

HRMS (ESI) Calcd for $\mathrm{C}_{27} \mathrm{H}_{27} \mathrm{Cl}_{2} \mathrm{~N}_{2} \mathrm{O}_{6} \mathrm{~S}^{+}[\mathrm{M}+\mathrm{H}]^{+}$577.0961; Found: 577.0957.

Chiral HPLC spectrum of racemic $4 \mathbf{e}$ 


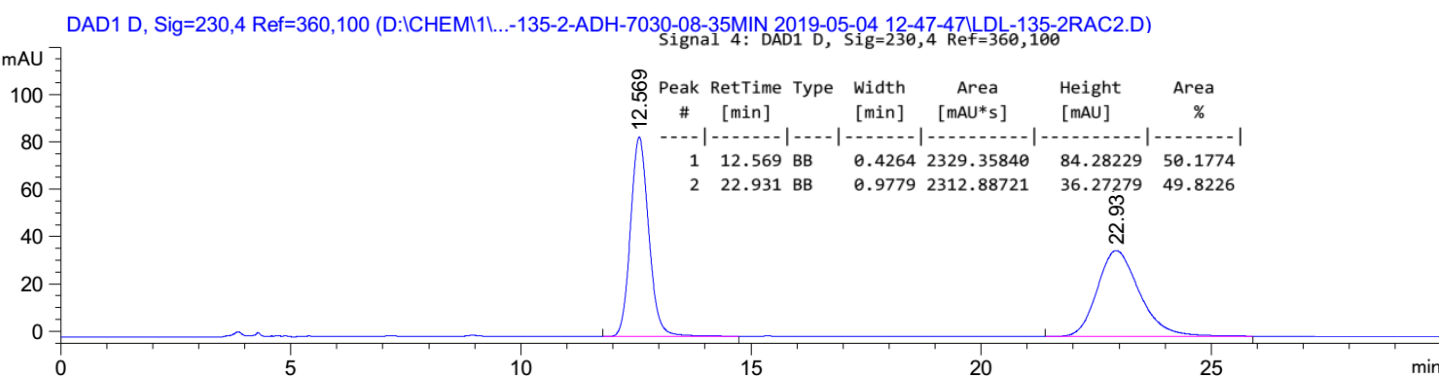

Chiral HPLC spectrum of $(S)-4 e$

DAD1 D, Sig=230,4 Ref=360,100 (D:ICHEMI1।...LLL-135-2-ADH-7030-08-35MIN 2019-05-04 12-47-47/LDL-135-2.D)
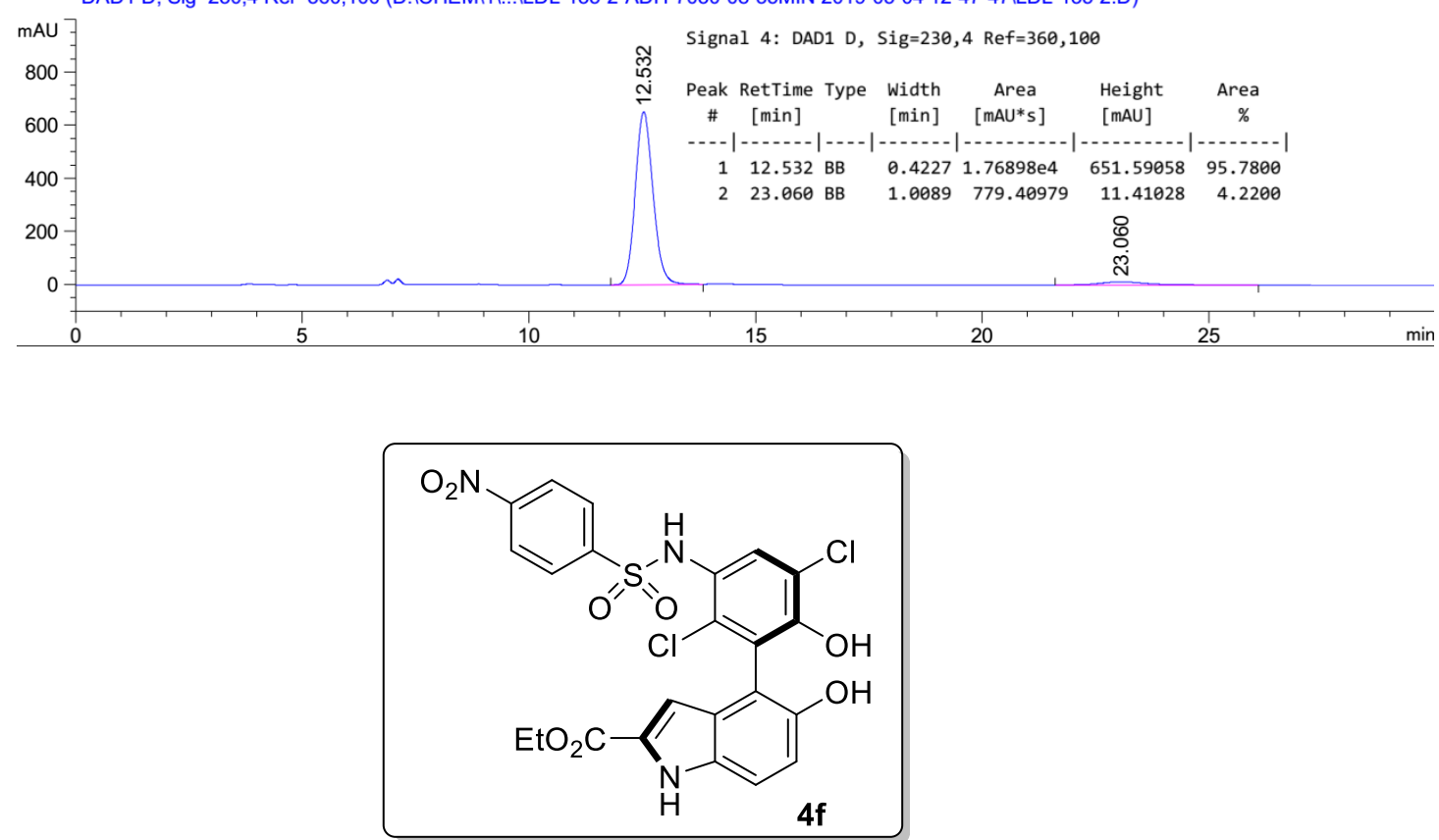

ethyl (S)-4-(2,5-dichloro-6-hydroxy-3-((4-nitrophenyl)sulfonamido)phenyl)-5-hydroxy$1 H$-indole-2-carboxylate

Pale yellow solid; Yield: $79 \mathrm{mg}, 70 \%$ (petroleum ether/ethyl acetate $=3: 2$ ); ee $=92 \%$; $[\alpha]_{\mathrm{D}}^{26}=$ $30.0(\mathrm{c}=0.2, \mathrm{MeOH})$.

HPLC analysis: HPLC DAICEL CHIRALCEL IG, hexane/isopropanol $(0.1 \%$ TFA $)=60 / 40$, $0.8 \mathrm{~mL} / \mathrm{min}, \lambda=230 \mathrm{~nm}, \mathrm{t}_{\mathrm{R}}($ minor $)=13.3 \mathrm{~min}, \mathrm{t}_{\mathrm{R}}($ major $)=31.4 \mathrm{~min}$.

${ }^{1}$ H NMR (400 MHz, Acetone-d $\left._{6}\right) \delta 10.77$ (s, 1H), 8.97 (s, 1H), $8.44-8.37$ (m, 2H), 8.35 (s, 1H), $8.10-8.01(\mathrm{~m}, 2 \mathrm{H}), 7.94(\mathrm{~s}, 1 \mathrm{H}), 7.70(\mathrm{~s}, 1 \mathrm{H}), 7.41(\mathrm{dd}, J=8.9,0.6 \mathrm{~Hz}, 1 \mathrm{H}), 6.98(\mathrm{~d}, J$ $=8.9 \mathrm{~Hz}, 1 \mathrm{H}), 6.51(\mathrm{~d}, J=1.5 \mathrm{~Hz}, 1 \mathrm{H}), 4.38(\mathrm{~m}, 2 \mathrm{H}), 1.37(\mathrm{t}, J=7.1 \mathrm{~Hz}, 3 \mathrm{H})$.

${ }^{13}$ C NMR (100 MHz, Acetone-d $) \delta=162.12,152.33,151.17,150.00,146.59,133.13$, $131.77,130.15,129.54,129.11,128.48,126.17,125.49,125.16,120.26,117.33,114.85$, $111.27,106.77,61.34,14.62$.

HRMS (ESI) Calcd for $\mathrm{C}_{23} \mathrm{H}_{18} \mathrm{Cl}_{2} \mathrm{~N}_{3} \mathrm{O}_{8} \mathrm{~S}^{+}[\mathrm{M}+\mathrm{H}]^{+}$566.0186; Found: 566.0181 .

Chiral HPLC spectrum of racemic $\mathbf{4 f}$ 


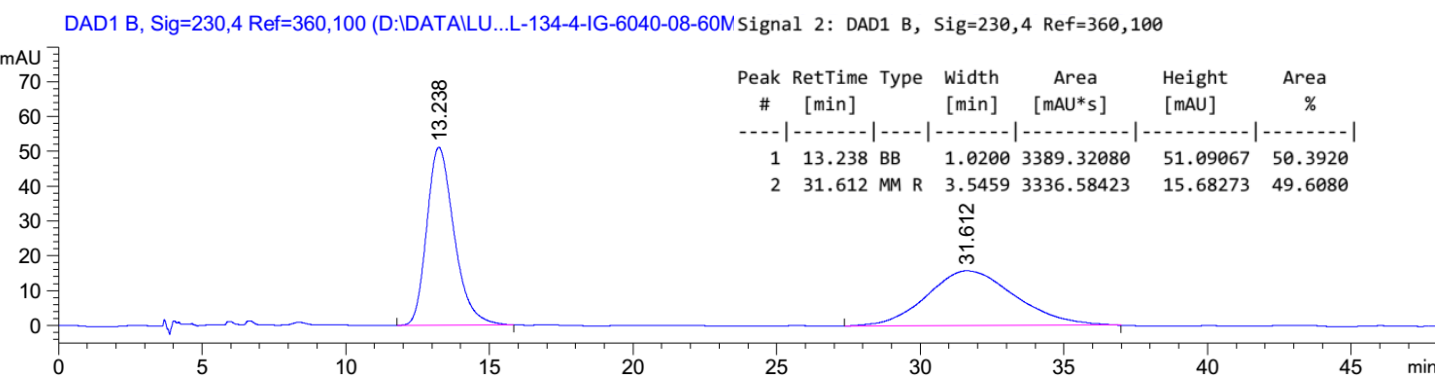

Chiral HPLC spectrum of $(S)-\mathbf{4 f}$

DAD1 B, Sig=230,4 Ref=360,100 (D:IDATAILU...GILDL-134-4-IG-6040-08-60MIN 2019-05-03 11-33-10ILDL-134-4.D)
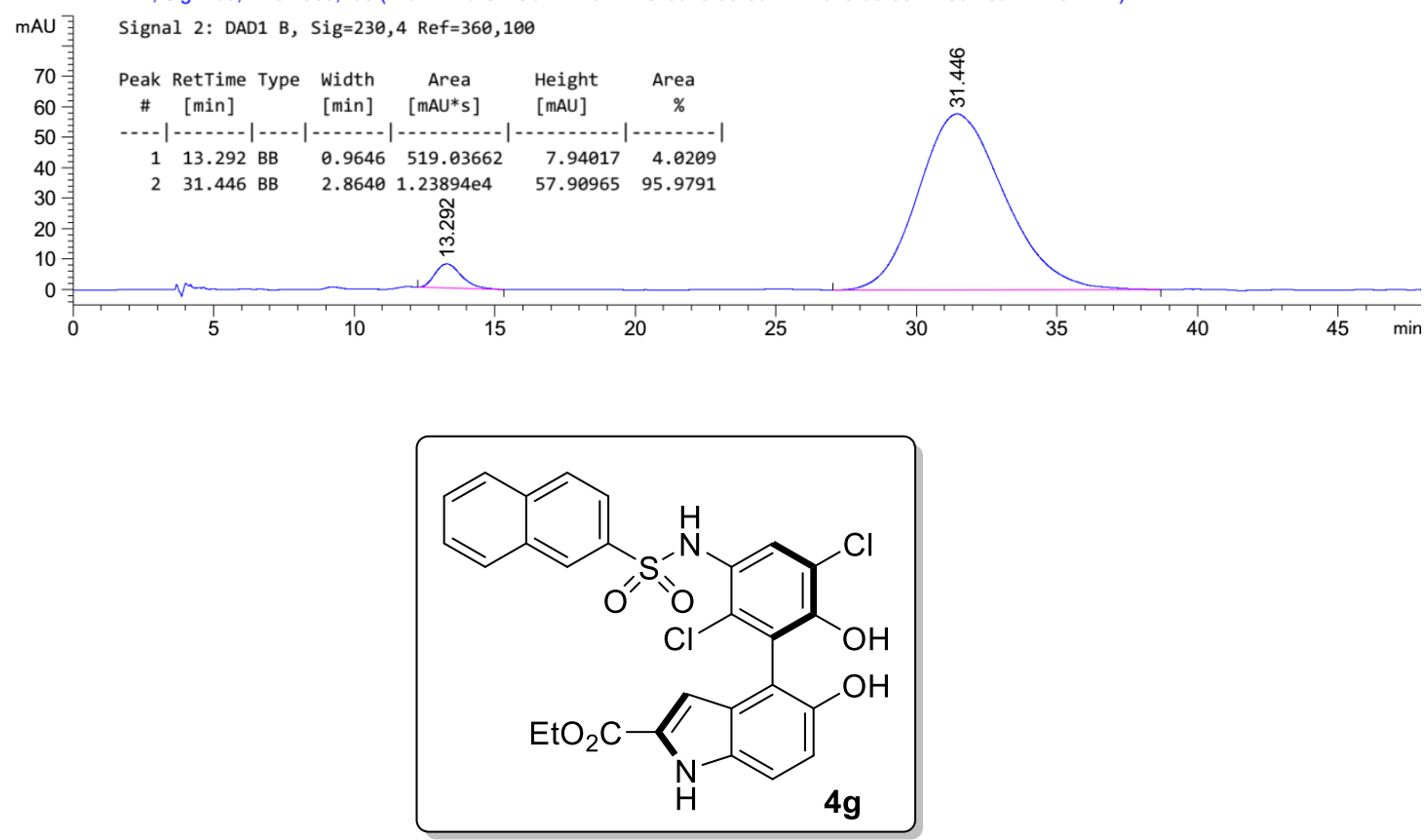

ethyl (S)-4-(2,5-dichloro-6-hydroxy-3-(naphthalene-1-sulfonamido)phenyl)-5-hydroxy$1 H$-indole-2-carboxylate

Pale yellow solid; Yield: $83 \mathrm{mg}, 73 \%$ (petroleum ether/ethyl acetate $=3: 2$ ); ee $=91 \%$; $[\alpha]_{\mathrm{D}}^{26}=$ $35.4(\mathrm{c}=0.2, \mathrm{MeOH})$.

HPLC analysis: HPLC DAICEL CHIRALCEL IB, hexane/isopropanol $(0.1 \%$ TFA $)=60 / 40$, $0.8 \mathrm{~mL} / \mathrm{min}, \lambda=230 \mathrm{~nm}, \mathrm{t}_{\mathrm{R}}($ major $)=9.9 \mathrm{~min}, \mathrm{t}_{\mathrm{R}}($ minor $)=15.3 \mathrm{~min}$.

${ }^{1} \mathrm{H}$ NMR (400 MHz, CDCl $) \delta 9.16(\mathrm{~s}, 1 \mathrm{H}), 8.62(\mathrm{~d}, J=8.5 \mathrm{~Hz}, 1 \mathrm{H}), 8.18(\mathrm{~d}, J=7.3 \mathrm{~Hz}, 1 \mathrm{H})$, $8.04(\mathrm{~d}, J=8.2 \mathrm{~Hz}, 1 \mathrm{H}), 7.86(\mathrm{~d}, J=8.1 \mathrm{~Hz}, 1 \mathrm{H}), 7.68(\mathrm{~s}, 1 \mathrm{H}), 7.63-7.41(\mathrm{~m}, 3 \mathrm{H}), 7.25(\mathrm{~d}, J$ $=9.2 \mathrm{~Hz}, 1 \mathrm{H}), 7.09(\mathrm{~s}, 1 \mathrm{H}), 6.91(\mathrm{~d}, J=8.9 \mathrm{~Hz}, 1 \mathrm{H}), 6.30(\mathrm{~s}, 1 \mathrm{H}), 5.74(\mathrm{~s}, 1 \mathrm{H}), 5.08(\mathrm{~s}, 1 \mathrm{H})$, $4.33(\mathrm{q}, J=7.1 \mathrm{~Hz}, 2 \mathrm{H}), 1.37(\mathrm{t}, J=7.1 \mathrm{~Hz}, 3 \mathrm{H})$.

${ }^{13}$ C NMR (100 MHz, $\left.\mathbf{C D C l}_{3}\right) \delta=161.91,148.73,147.76,135.35,134.29,133.96,132.12$, $130.23,129.28,128.54,128.47,128.37,127.28,127.14,126.82,125.54,124.35,124.19$, $121.85,119.93,116.79,114.50,109.97,106.71,61.44,14.51$.

HRMS (ESI) Calcd for $\mathrm{C}_{27} \mathrm{H}_{21} \mathrm{Cl}_{2} \mathrm{~N}_{2} \mathrm{O}_{6} \mathrm{~S}^{+}[\mathrm{M}+\mathrm{H}]^{+}$571.0492; Found: 571.0490 . 
Chiral HPLC spectrum of racemic $\mathbf{4 g}$

DAD1 B, Sig=230,4 Ref=360,100 (D:IDATAILU...DL-134-6-IB-6040-08-60MIN 2019-05-03 09-47-51ILDL-134-6RAC.D)

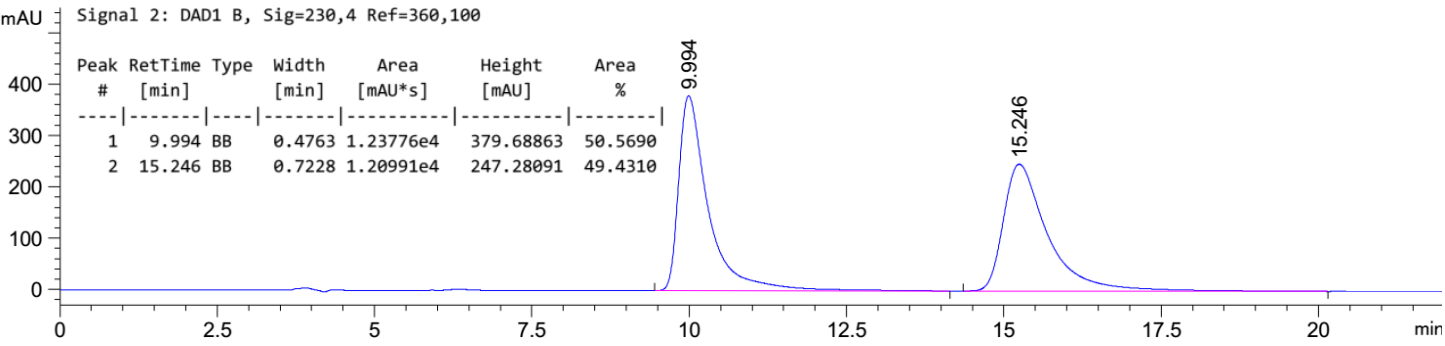

Chiral HPLC spectrum of $(S)-\mathbf{4 g}$

DAD1 B, Sig=230,4 Ref=360,100 (D:IDATAILU...GILDL-134-6-IB-6040-08-60MIN 2019-05-03 09-47-51 ILDL-134-6.D)

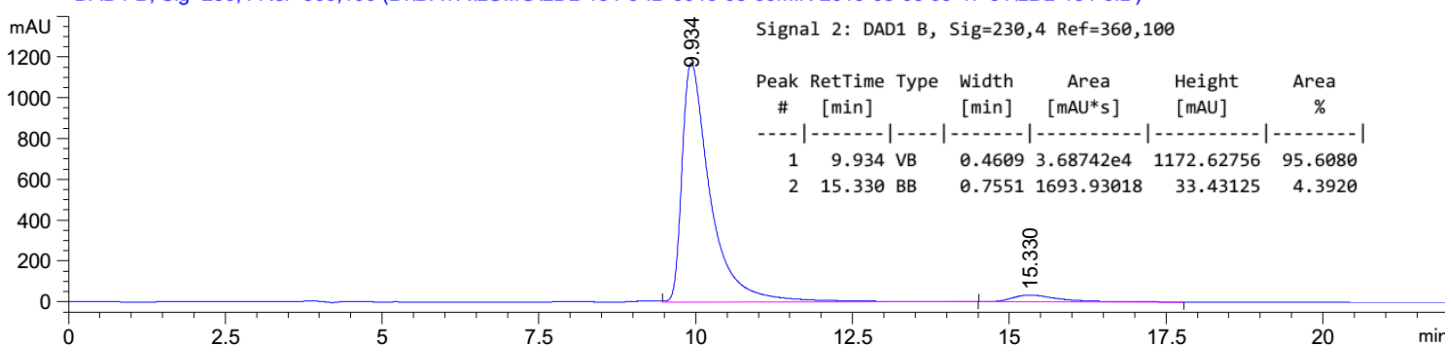




\section{Experimental measurement of enantiomerization}

\section{barrier for $3 a$ and $4 a$}

The enantiomerization barrier, corresponding to barrier to rotation for $\mathbf{3 a}$ and $\mathbf{4 a}$ atropisomers, was obtained by kinetic of racemization of an enantiomer via chiral HPLC analysis. The slope of the first-order kinetic line gives the racemization constant $(\mathrm{K}$ racemization $=2 \mathrm{x} \mathrm{K}$ enantiomerzation). Eyring equation gives the enatiomerezation barrier from enatiomerization constant ( $\mathrm{k}$ enantiomerization), $\mathrm{R}=8.31451 \mathrm{~J} \cdot \mathrm{K}^{-1} \cdot \mathrm{mol}^{-1}, \mathrm{~h}=6.62608 \times 10^{-34} \mathrm{~J} . \mathrm{s}$ and $\mathrm{k}_{\mathrm{B}}=$ $1.3806610^{-23} \mathrm{~J} . \mathrm{K}^{-1}$.

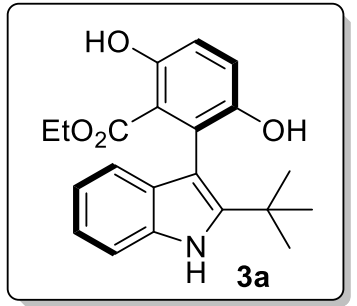

Solvent: $i-\mathrm{PrOH}$

Temperature $=80^{\circ} \mathrm{C}$

\begin{tabular}{|c|c|c|}
\hline Time $(\mathrm{min})$ & \% second eluted enantiomer $(\% \mathrm{t})$ & $\ln \left((\% \mathrm{t}-50) /\left(\% \mathrm{t}_{0}-50\right)\right)$ \\
\hline 0 & 97.24 & 0 \\
\hline 240 & 96.951 & -0.060916498 \\
\hline 720 & 95.883 & -0.083926325 \\
\hline 2160 & 89.329 & -0.238058843 \\
\hline 2880 & 86.453 & -0.313997243 \\
\hline
\end{tabular}

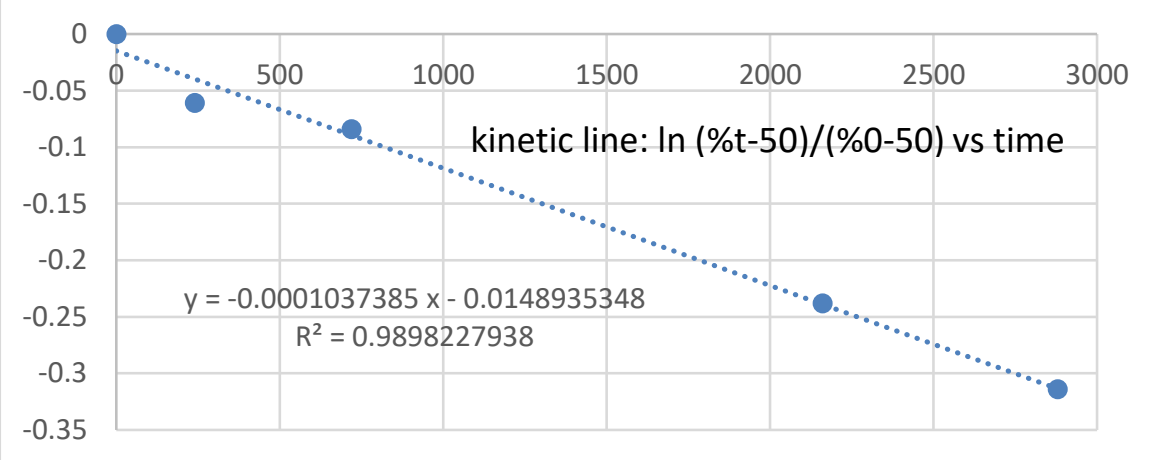

$\mathrm{K}$ racemization $=1.72897 \times 10^{-6} \mathrm{~S}^{-1}$

$\mathrm{K}$ enantiomerization $=8.6448750 \times 10^{-7} \mathrm{~S}^{-1}$

$\Delta \mathrm{G}$ enantiomerization $=128.0 \mathrm{KJ} \cdot \mathrm{mol}^{-1}$

Half-life time $\mathrm{t}_{1 / 2}=400901$ seconds

6682 minutes

111 hours

4.64 days

$\mathrm{T}=25^{\circ} \mathrm{C}, \mathrm{t}_{1 / 2}=46.9$ years 


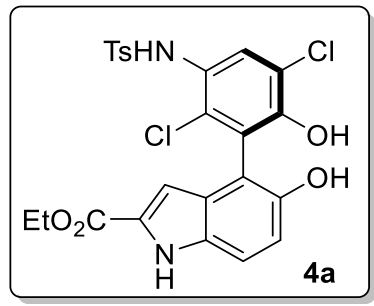

Solvent: $i-\mathrm{PrOH}$

Temperature $=80^{\circ} \mathrm{C}$

\begin{tabular}{|c|c|c|}
\hline Time (min) & \% second eluted enantiomer (\%t) & $\ln ((\% \mathrm{t}-50) /(\% \mathrm{t} 0-50))$ \\
\hline 0 & 95.525 & 0 \\
\hline 300 & 94.438 & -0.115926043 \\
\hline 720 & 90.516 & -0.208324045 \\
\hline 1920 & 79.573 & -0.52315922 \\
\hline 2880 & 69.408 & -0.944335651 \\
\hline
\end{tabular}

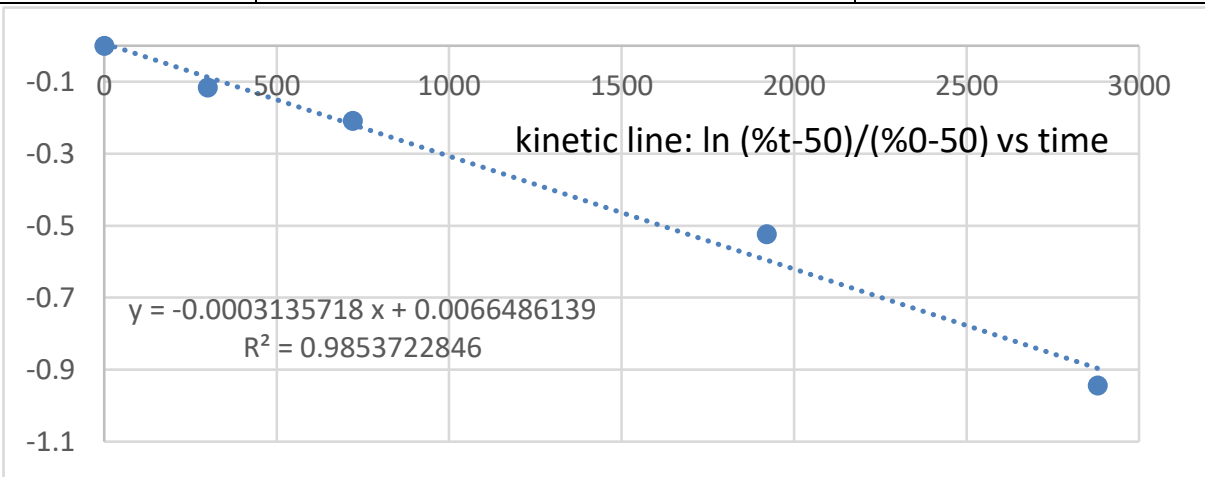

$\mathrm{K}$ racemization $=5.2261967 \times 10^{-6} \mathrm{~S}^{-1}$

$\mathrm{K}$ enantiomerization $=2.6130983 \times 10^{-6} \mathrm{~S}^{-1}$

$\Delta \mathrm{G}$ enantiomerization $=124.7 \mathrm{KJ} \cdot \mathrm{mol}^{-1}$

Half-life time $\mathrm{t}_{1 / 2}=132629$ seconds

2210 minutes

36.8 hours

1.54 days

$\mathrm{T}=25{ }^{\circ} \mathrm{C}, \mathrm{t}_{1 / 2}=12.6$ years

The enantiomerisation barrier determination of the product was studied by heating a solution of 3a $(0.05 \mathrm{mmol})$ in $i-\mathrm{PrOH}(2 \mathrm{~mL})$.

The enantiomerisation barrier determination of the product was studied by heating a solution of 4a $(0.02 \mathrm{mmol})$ in $i$-PrOH $(2 \mathrm{~mL})$. 


\section{X-ray crystal structural parameters of absolute configuration of $3 e$ and $3 q$}

Compound 3e (The crystal structure of compound $3 \mathrm{e}$ has been deposited at the Cambridge Crystallographic Data Centre (CCDC 1919874)).

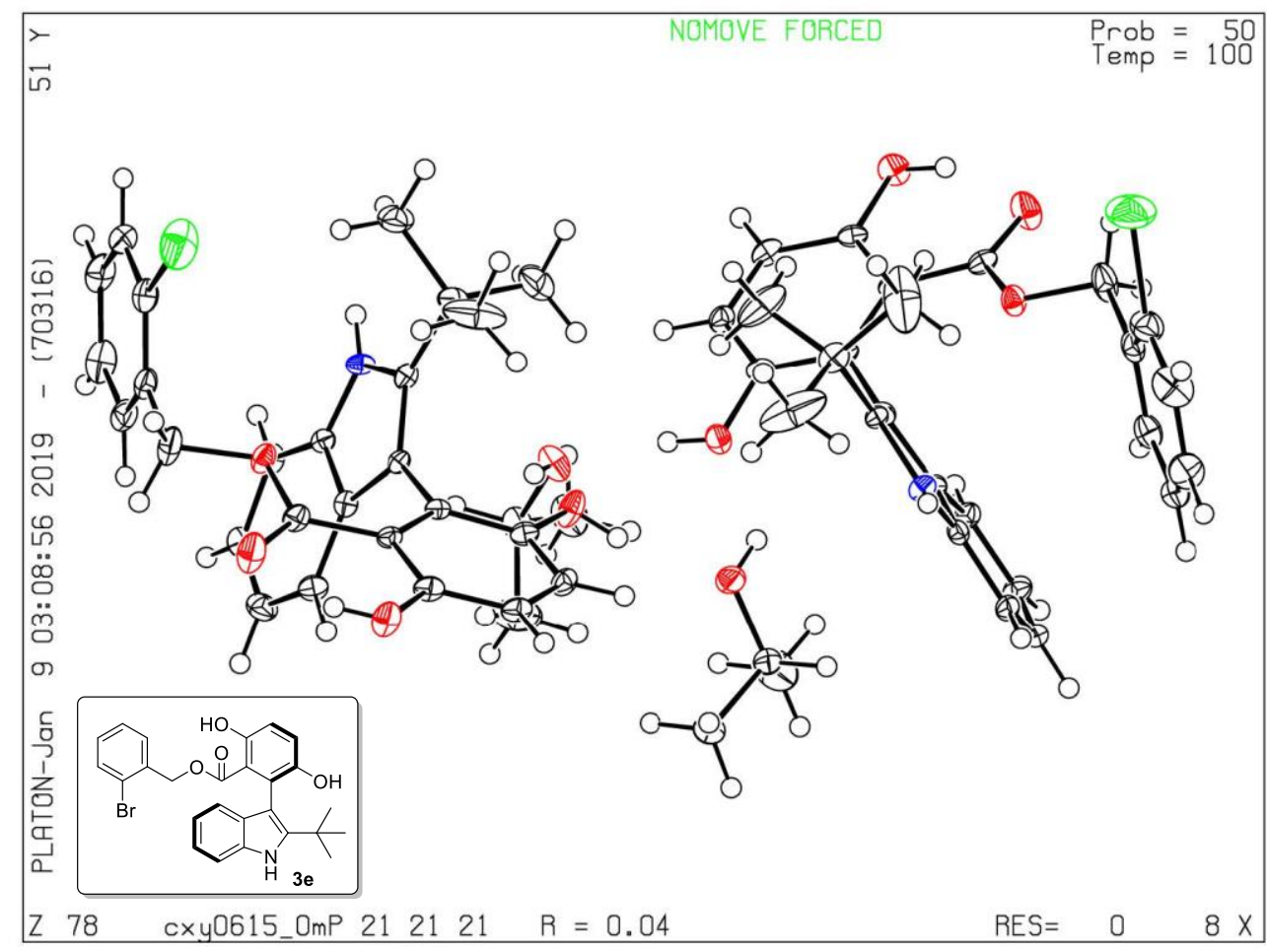

$\begin{array}{ll}\text { Identification code } & \text { 3e } \\ \text { Empirical formula } & \text { C29H32BrNO5 } \\ \text { Formula weight } & 554.46 \\ \text { Temperature/K } & 100 \\ \text { Crystal system } & \text { orthorhombic } \\ \text { Space group } & \text { P212121 } \\ \mathrm{a} / \AA & 9.8391(7) \\ \mathrm{b} / \AA & 18.4735(13) \\ \mathrm{c} / \AA & 29.010(2) \\ \alpha /^{\circ} & 90 \\ \beta / /^{\circ} & 90 \\ \gamma /{ }^{\circ} & 90\end{array}$


Volume/Å3

Z

pcalcg/cm3

$\mu / \mathrm{mm}-1$

$\mathrm{F}(000)$

Crystal size/mm3

Radiation

$2 \Theta$ range for data collection $/{ }^{\circ} 4.372$ to 52.834

Index ranges

$-12 \leq \mathrm{h} \leq 12,-23 \leq \mathrm{k} \leq 23,-36 \leq 1 \leq 36$

Reflections collected

74088

Independent reflections

10833 [Rint $=0.0738$, Rsigma $=0.0466]$

Data/restraints/parameters

Goodness-of-fit on F2

(33/0/666

1.052

Final $\mathrm{R}$ indexes $[\mathrm{I}>=2 \sigma(\mathrm{I})] \quad \mathrm{R} 1=0.0411, \mathrm{wR} 2=0.0886$

Final R indexes [all data] $\quad \mathrm{R} 1=0.0537, \mathrm{wR} 2=0.0945$

Largest diff. peak/hole / e -3 1.11/-1.09

Flack parameter $-0.005(3)$

Compound 3q (The crystal structure of compound $3 q$ has been deposited at the Cambridge Crystallographic Data Centre (CCDC 1919875)). 


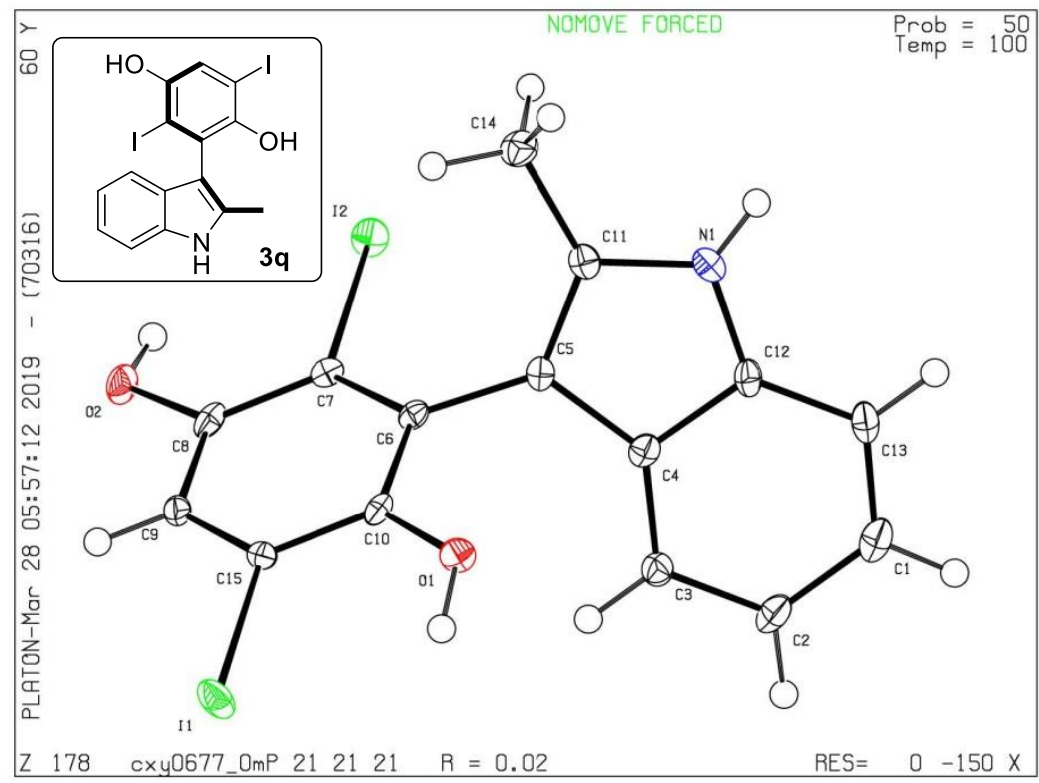

Identification code

3q

Empirical formula

$\mathrm{C}_{15} \mathrm{H}_{11} \mathrm{I}_{2} \mathrm{NO}_{2}$

Formula weight

491.05

Temperature/K

100.04

Crystal system

orthorhombic

Space group

$\mathrm{P} 2{ }_{1} 2_{1} 2_{1}$

$\mathrm{a} / \AA ̊$

$9.2030(5)$

$\mathrm{b} / \AA$

$10.3766(5)$

$\mathrm{c} / \AA$

15.6185(8)

$\alpha /^{\circ}$

90

$\beta /{ }^{\circ}$

90

$\gamma /{ }^{\circ}$

90

Volume $/ \AA^{3}$

1491.50(13)

Z

4

$\rho_{\text {calc }} \mathrm{g} / \mathrm{cm}^{3}$

2.187

$\mu / \mathrm{mm}^{-1}$

4.217

$\mathrm{F}(000)$

920.0

Crystal size $/ \mathrm{mm}^{3}$

$0.38 \times 0.34 \times 0.29$

Radiation

$\operatorname{MoK} \alpha(\lambda=0.71073)$

$2 \Theta$ range for data collection $/{ }^{\circ} 5.138$ to 55.048

Index ranges

$-11 \leq \mathrm{h} \leq 11,-12 \leq \mathrm{k} \leq 13,-20 \leq 1 \leq 17$

Reflections collected

20787

Independent reflections

$3435\left[R_{\text {int }}=0.0641, R_{\text {sigma }}=0.0355\right]$

Data/restraints/parameters

$3435 / 0 / 185$

Goodness-of-fit on $\mathrm{F}^{2}$

1.079

Final R indexes $[\mathrm{I}>=2 \sigma(\mathrm{I})]$

$\mathrm{R}_{1}=0.0217, \mathrm{wR}_{2}=0.0522$ 
Final $\mathrm{R}$ indexes [all data] $\quad \mathrm{R}_{1}=0.0222, \mathrm{wR}_{2}=0.0524$

Largest diff. peak/hole / e $\AA^{-3} 1.02 /-0.75$

Flack parameter

$-0.021(13)$

Structural characterization and absolute configuration of $4 a$

(a) Key HMBC correlations for $4 \mathrm{a}$

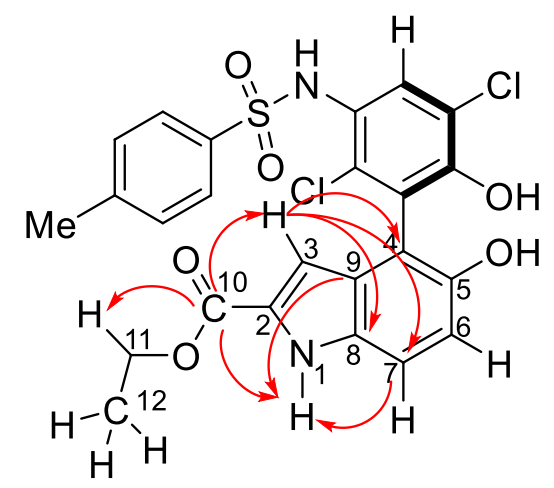



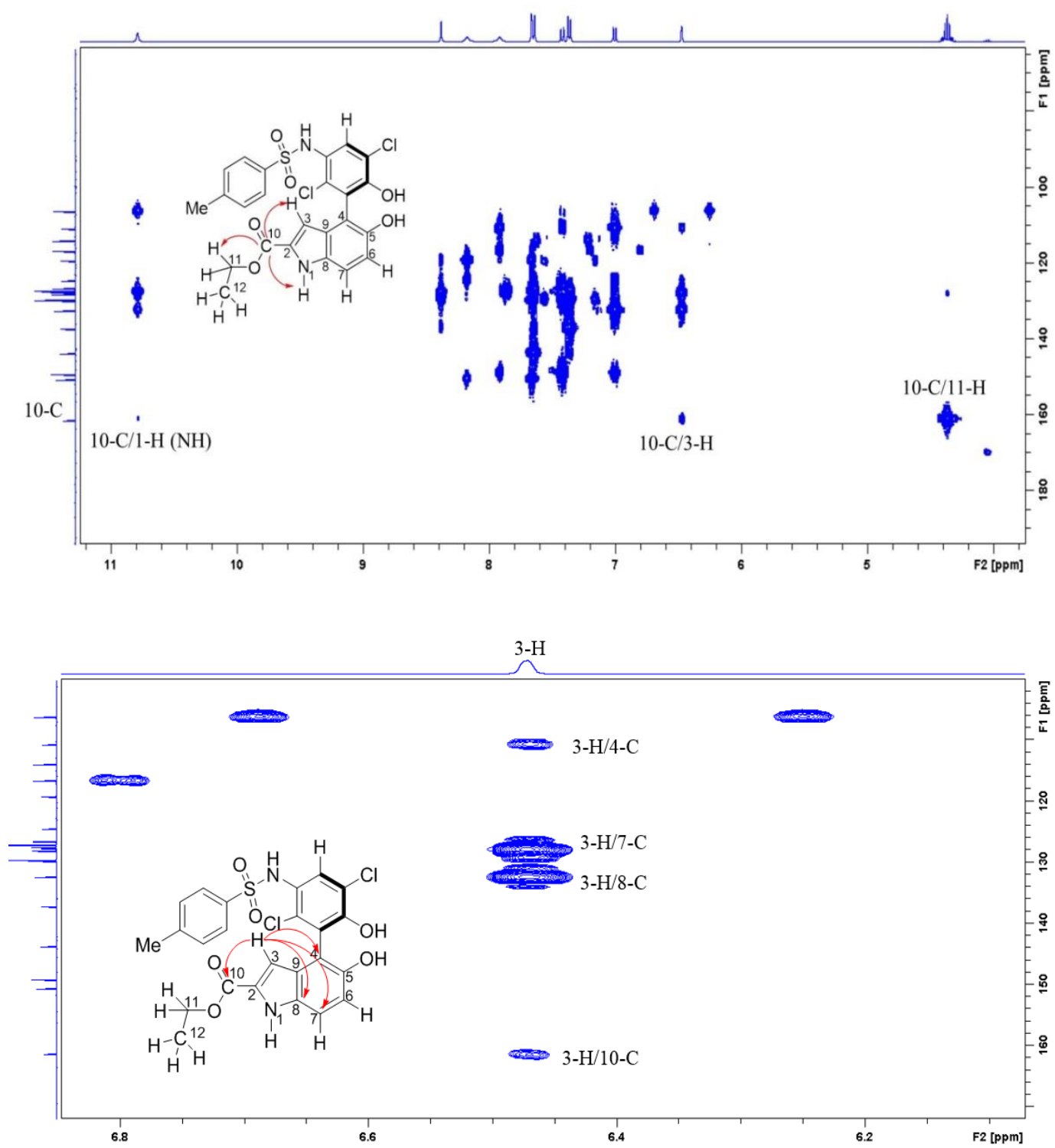

(b) Absolute configuration of $4 \mathbf{a}$

Compound $4 \mathbf{a}$ to be determined:<smiles></smiles>

Chemical Formula: $\mathrm{C}_{24} \mathrm{H}_{20} \mathrm{Cl}_{2} \mathrm{~N}_{2} \mathrm{O}_{6} \mathrm{~S}$

Exact Mass: 534.0419

Sample concentration: $3.121 \times 10^{-4} \mathrm{~mol} / \mathrm{L}($ in DCM)

Experimental (black) and Calculated (red) CD spectra. Calculations were performed on the 
methyl ester instead of the ethyl ester to reduce conformational possibilities. Ground state geometry optimizations and TD-DFT calculations are at the B3LYP/6-31G(d) level with the PCM solvation model. Computed spectrum has been red-shifted by $50 \mathrm{~nm}$ to better compare to experiments.

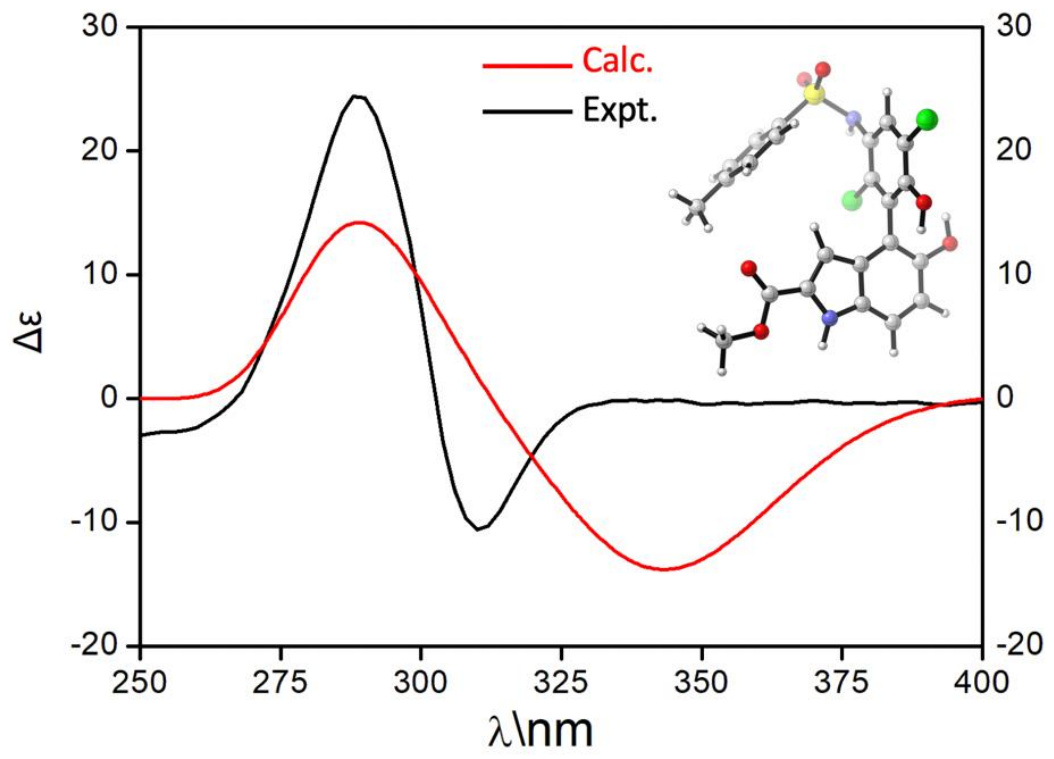

\section{Preparative Scale Experiment}<smiles>CCOC(=O)C1=CC(=O)C=CC1=O</smiles>

$5.0 \mathrm{mmol}$

$(0.90 \mathrm{~g})$<smiles>CC(C)(C)c1cc2ccccc2[nH]1</smiles>

$6.0 \mathrm{mmol}$

$(1.04 \mathrm{~g})$<smiles>CCOC(=O)c1c(O)ccc(O)c1-c1c(C(C)(C)C)[nH]c2ccccc12</smiles>

$(1.41 \mathrm{~g})$

To a $100 \mathrm{~mL}$ round-bottom flask with a magnetic stirring bar were sequentially added chiral phosphoric acid (S)-C5 (3 mol\%, $89 \mathrm{mg})$, 2-(tert-butyl)-1H-indole (6.0 mmol, $1.04 \mathrm{~g}$ ), and $\mathrm{CH}_{2} \mathrm{Cl}_{2}(40 \mathrm{~mL})$ under argon atmosphere. After stirring for $10 \mathrm{~min}$ at $-10{ }^{\circ} \mathrm{C}$, a solution of 
2-ethoxycarbonyl-1,4-benzoquinone $(5.0 \mathrm{mmol}, 0.90 \mathrm{~g})$ in DCM $(20 \mathrm{~mL})$ was added dropwise to the mixture. After stirring at $-10{ }^{\circ} \mathrm{C}$ for $18 \mathrm{~h}$, the mixture was concentrated and purified by silica gel column chromatography (petroleum ether/DCM $=2: 1$ to $1: 1$ ) to afford pure product $3 \mathbf{a}(1.41 \mathrm{~g}, 80 \%$ yield, $93 \%$ ee).

\section{Mechanism Investigation}

\section{(a) Control experiments}

To gain further insight into the reaction mechanism, we performed a series of control experiments. To verify the possible activation mode of CPA to the substrates, the 2-tert-butylindole 2a and N-methyl- protected 2-tert-butylindole 2s were employed in the reaction under standard reaction conditions (Scheme S2A), the sharply decreasing enantioselectivity after active $\mathrm{N}-\mathrm{H}$ of $\mathbf{2 a}$ is protected by methyl group, indicates that the forming $\mathrm{H}$-bonding interaction between chiral phosphoric acid and indole plays an important role for the sterochemical control during the construction of product $\mathbf{3}$. In vivid constrast, the enantioselectivity almost has no influence when the 5-hydroxyindole is protected for the transformations of substrates $\mathbf{2 r}$ (Scheme S2B). 


\section{Scheme S2 Control experiments}

$\boldsymbol{A}$<smiles>CCOC(=O)C1=CC(=O)C=CC1=O</smiles>

$1 \mathbf{a}$<smiles>[R]n1c(C(C)(C)C)cc2ccccc21</smiles>

2a, $\mathrm{R}=\mathrm{H}$

2s, $\mathrm{R}=\mathrm{Me}$
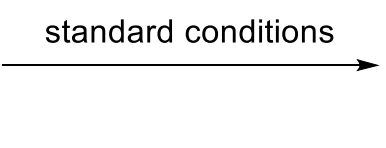

3a $\mathrm{R}=\mathrm{H}, 84 \%, 94 \%$ ee

3v $\mathrm{R}=\mathrm{Me}, 81 \%, 3 \%$ ee

B<smiles>[N+]=C1C=C(Cl)C(=O)C=C1Cl</smiles>

$1 \mathrm{k}$<smiles>[R]n1c(C)cc2cc(O)ccc21</smiles>

$2 r, R=B o c$ $\stackrel{\text { standard conditions }}{\longrightarrow}$

Pale yellow oil; $81 \%$ yield (petroleum ether $/ \mathrm{DCM}=4: 1$ ); ee $=3 \%$.

HPLC analysis: HPLC DAICEL CHIRALCEL IA, hexane/isopropanol $=95 / 5,0.4 \mathrm{~mL} / \mathrm{min}$, $\lambda=214 \mathrm{~nm}, \mathrm{t}_{\mathrm{R}}($ minor $)=18.3 \mathrm{~min}, \mathrm{t}_{\mathrm{R}}($ major $)=20.2 \mathrm{~min}$.

${ }^{1}$ H NMR (400 MHz, CDCl $) \delta 10.82(\mathrm{~s}, 1 \mathrm{H}), 7.30(\mathrm{~d}, J=8.3 \mathrm{~Hz}, 1 \mathrm{H}), 7.24-7.16(\mathrm{~m}, 1 \mathrm{H})$, $7.14(\mathrm{~d}, J=9.0 \mathrm{~Hz}, 1 \mathrm{H}), 7.03-6.91(\mathrm{~m}, 3 \mathrm{H}), 5.02(\mathrm{~s}, 1 \mathrm{H}), 3.99(\mathrm{~s}, 3 \mathrm{H}), 3.87(\mathrm{~m}, 1 \mathrm{H}), 3.76$ (m, 1H), $1.37(\mathrm{~s}, 9 \mathrm{H}), 0.36(\mathrm{t}, J=7.1 \mathrm{~Hz}, 3 \mathrm{H})$.

${ }^{13}$ C NMR (100 MHz, CDCl $) \delta=170.98,156.46,147.40,144.09,138.46,128.76,123.54$, $122.26,121.75,120.03,118.86,118.16,113.10,108.47,105.41,60.87,34.17,33.50,30.73$, 12.72 .

Racemic 3v

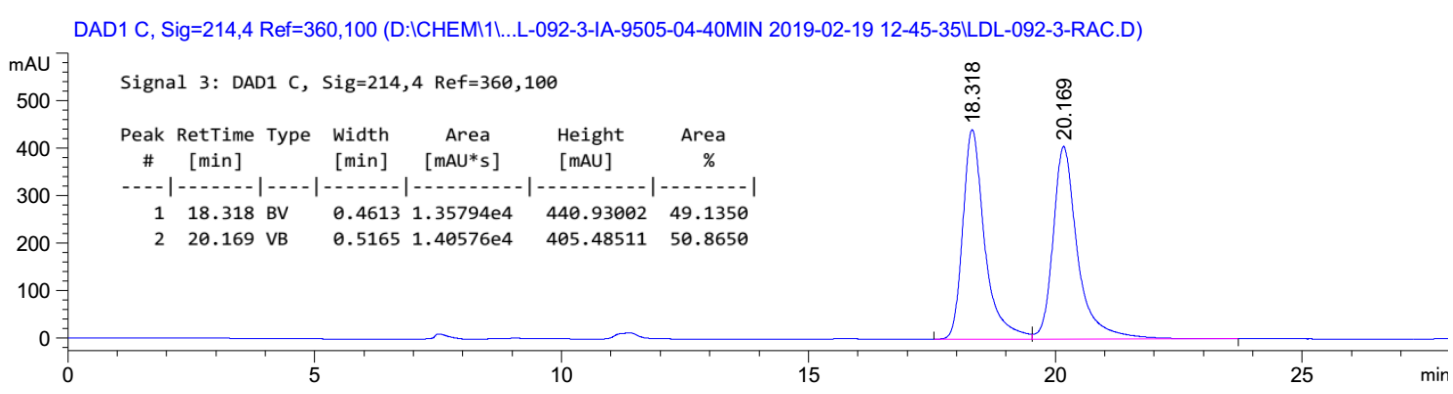

Chiral 3v 
DAD1 C, Sig=214,4 Ref=360,100 (D:ICHEMI1।...LILDL-092-3-IA-9505-04-40MIN 2019-02-19 12-45-35ILDL-092-3.D)

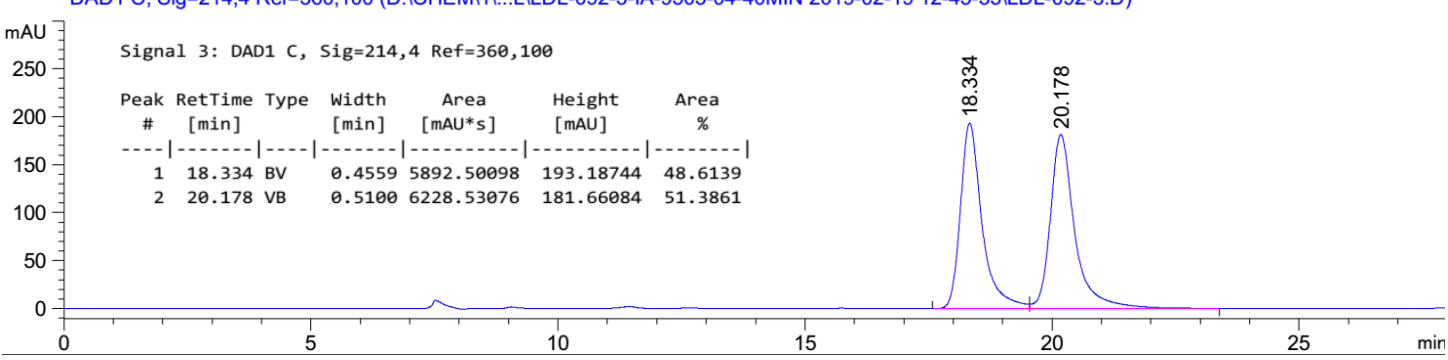

$\stackrel{\stackrel{m}{\infty}}{\stackrel{0}{1}}$
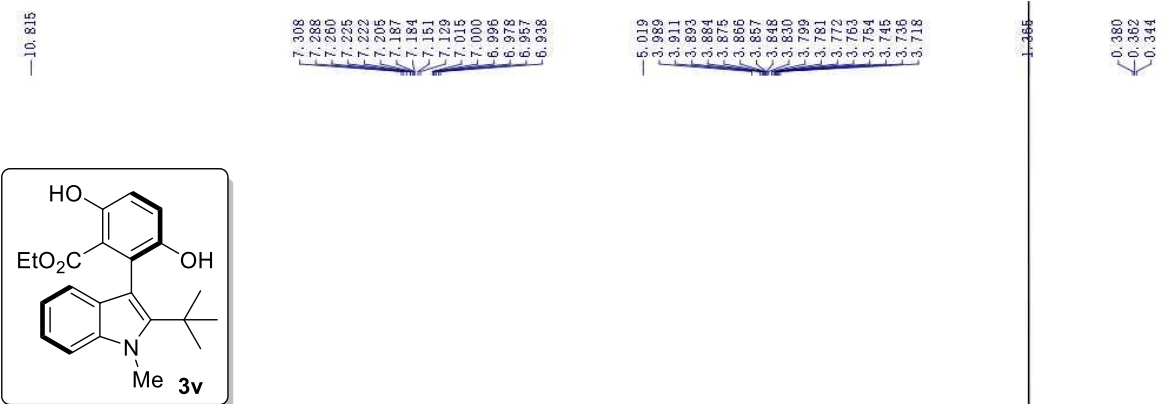

Me 3v

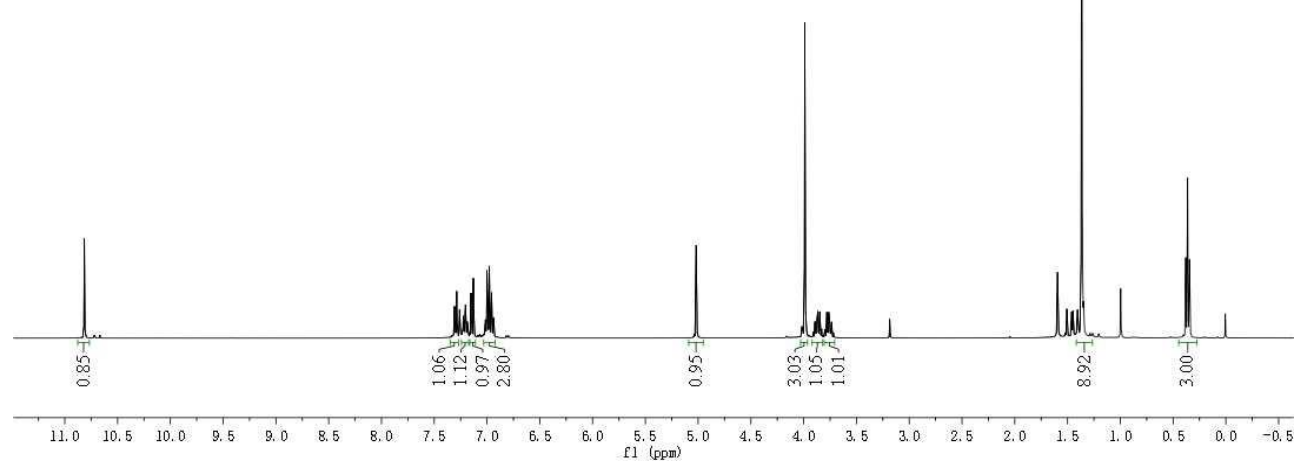

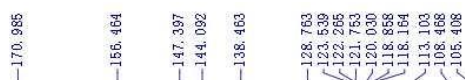

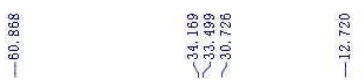

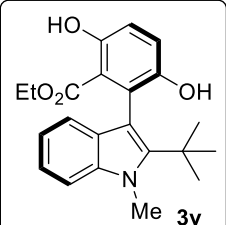

Me 3v
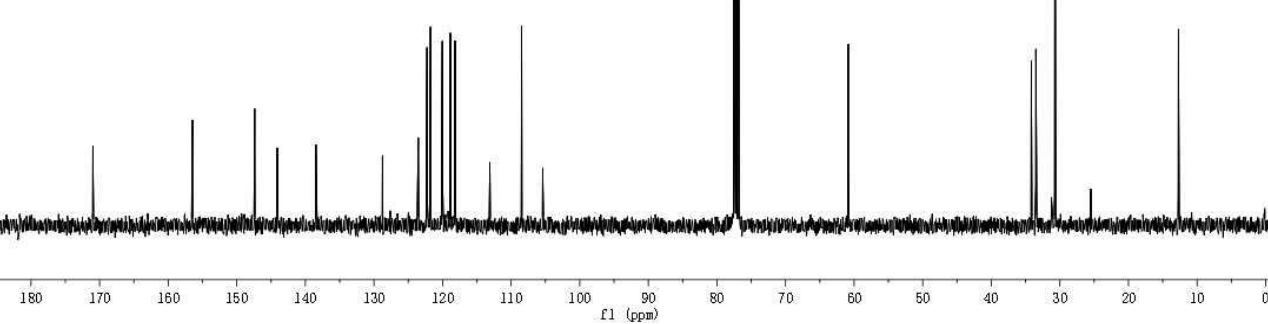


\section{(b) Proposed reaction process}

On the basis of above experimental results and related reports on central-to-axial chirality conversion (Wettlaufer, D. G. et al. J. Am. Chem. Soc. 1984, 106, 1135), plausible pathway is depicted in Scheme S3. During the forming process of products 3 or 4 , the reactions should initially generate intermediates $A^{\prime}$ or $B^{\prime}$ through enantioselective conjugate additions of indoles to the quinones under chiral phosphoric acid. In the following step, aromatization with central-to-axial chirality transfer affords the desired axially chiral products. The excellent enantioselectivities are attributed to the simultaneously interaction that chiral phosphoric acid acts as a bifunctional catalyst to activate indoles and quinones through a dual H-bonding activation mode. However, a cascade process that involves aminal formation, sigmatropic rearrangement, and aromatization cannot be ruled out at this stage (Kürti, L. et al. J. Am. Chem. Soc. 2016, 138, 5202; Angew. Chem. Int. Ed. 2016, 55, 566.).

Scheme S4 Proposed reaction process
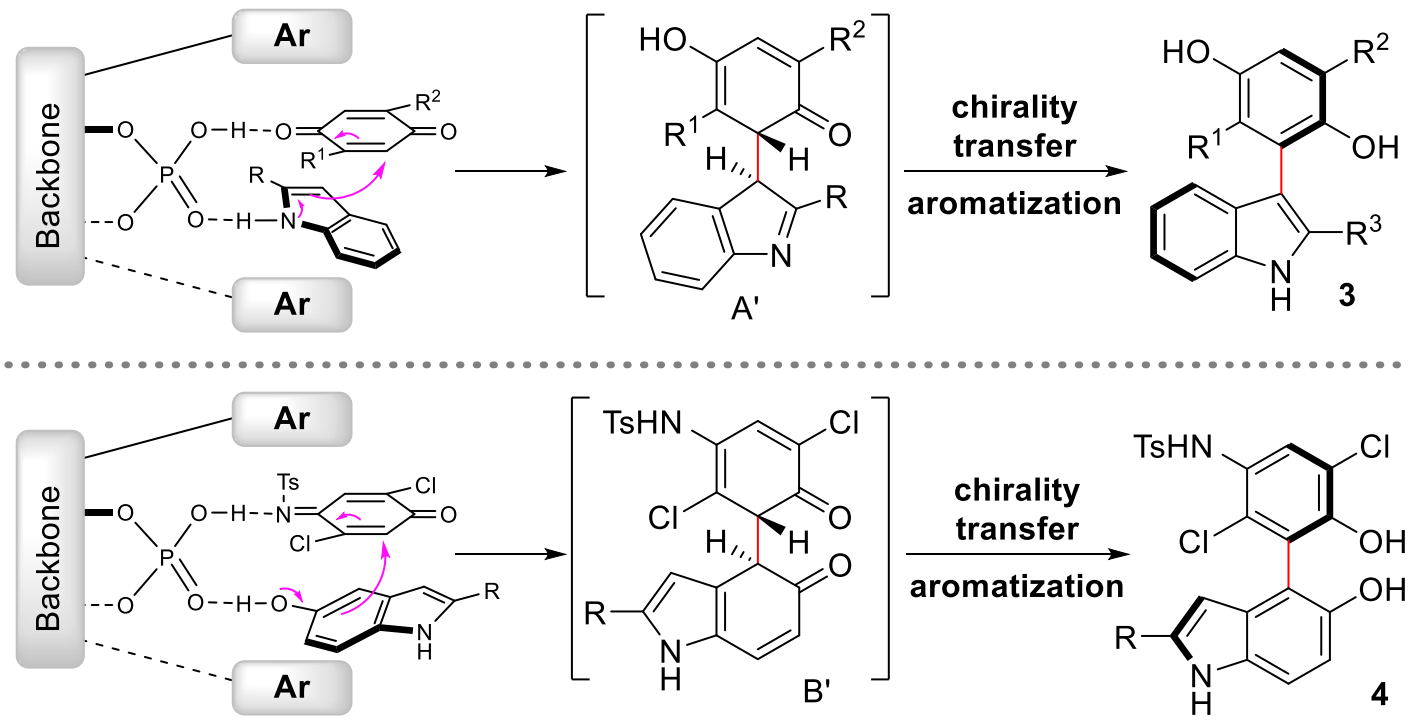


\section{Application of 4a in addition of diethylzinc to aldehyde}

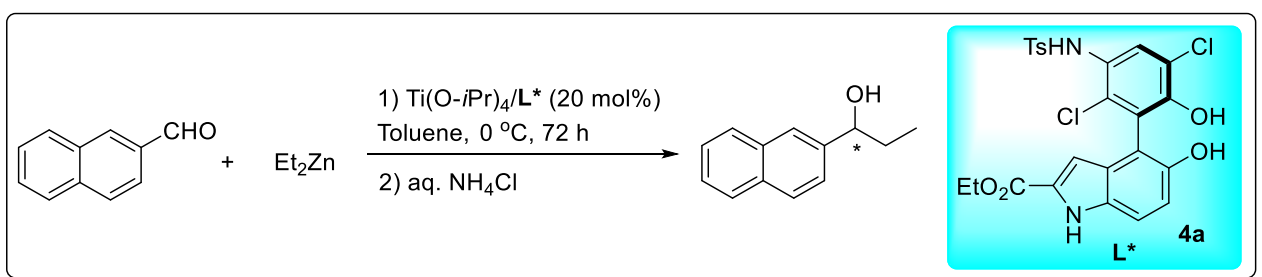

The operations were carried out in extra dry solvent under an argon atmosphere. $89 \mu \mathrm{L}$ of $\mathrm{Ti}(\mathrm{O} i \mathrm{Pr})_{4}(0.3 \mathrm{mmol})$ was added to a suspension of chiral ligand $4 \mathbf{a}(0.04 \mathrm{mmol})$ in $0.5 \mathrm{~mL}$ of toluene, and the mixture was stirred for $1 \mathrm{~h}$ at room temperature. After being cooled to $0{ }^{\circ} \mathrm{C}$, $0.6 \mathrm{mmol}$ of $\mathrm{Et}_{2} \mathrm{Zn}$ ( $1 \mathrm{M}$ solution in toluene) was added to the system. After $2 \mathrm{~h}$, 2-naphthylaldehyde $(0.2 \mathrm{mmol})$ in $1 \mathrm{~mL}$ toluene was added and the mixture was further 
stirred at the same temperature for $72 \mathrm{~h}$. The reaction was then quenched with saturated $\mathrm{NH}_{4} \mathrm{Cl}$ solution, filtered through a glass fiber filter, extracted with ethyl acetate, and dried over $\mathrm{Na}_{2} \mathrm{SO}_{4}$. After evaporation of the solvent, the residue was purified by column chromatography on silica gel to afford the corresponding product.

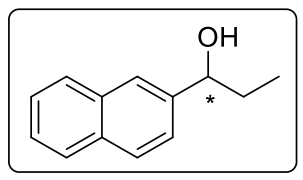

Faint yellow oil; Yield: $26 \mathrm{mg}, 70 \%$; ee $=65 \% ;[\alpha]_{\mathrm{D}}^{26}=-40.0\left(\mathrm{c}=0.2, \mathrm{CHCl}_{3}\right)$.

${ }^{1}$ H NMR (400 MHz, CDCl $) \delta 7.84(\mathrm{~m}, 3 \mathrm{H}), 7.76(\mathrm{~s}, 1 \mathrm{H}), 7.48(\mathrm{~m}, 3 \mathrm{H}), 4.74(\mathrm{t}, J=6.6 \mathrm{~Hz}$, $1 \mathrm{H}), 2.37(\mathrm{~s}, 1 \mathrm{H}), 2.03-1.74(\mathrm{~m}, 2 \mathrm{H}), 0.95(\mathrm{t}, J=7.4 \mathrm{~Hz}, 3 \mathrm{H})$.

${ }^{13}$ C NMR (100 MHz, $\left.\mathbf{C D C l}_{3}\right) \delta=142.02,133.34,133.05,128.28,128.01,127.76,126.17$, $125.83,124.81,124.25,76.14,31.81,10.23$.

HPLC analysis: HPLC DAICEL CHIRALCEL OD-H, hexane/isopropanol $=96 / 4,1.0$ $\mathrm{mL} / \min , \lambda=230 \mathrm{~nm}, \mathrm{t}_{\mathrm{R}}($ major $)=20.0 \mathrm{~min}, \mathrm{t}_{\mathrm{R}}($ minor $)=21.8 \mathrm{~min}$.

\section{Racemic}

DAD1 D, Sig=230,4 Ref=360,100 (D:IDATAILUDONGLIANGILDL-20190524 2019-05-24 07-38-09LLDL-0524-1-RAC2.D)

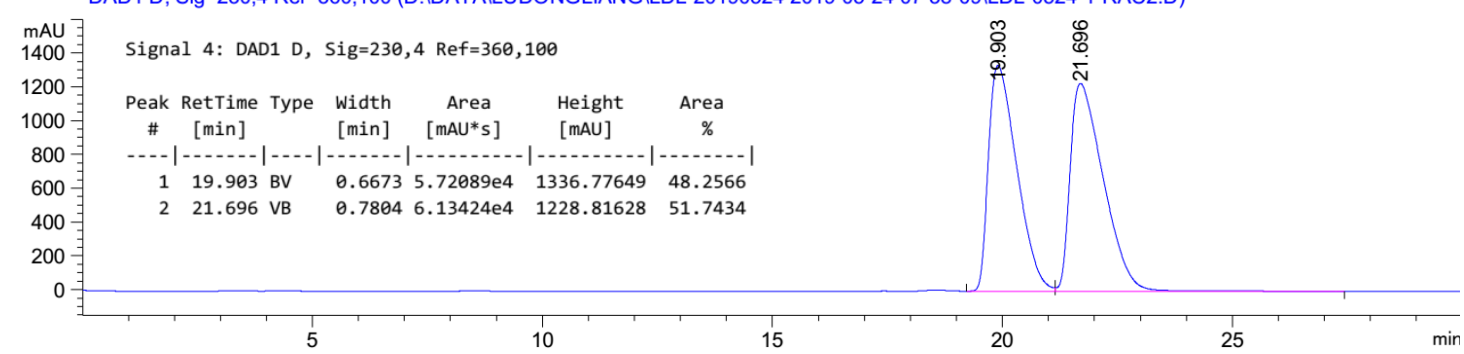

Chiral

DAD1 D, Sig=230,4 Ref=360,100 (D:IDATAILUDONGLIANGILDL-20190524 2019-05-24 07-38-09ILDL-0524-1.D)

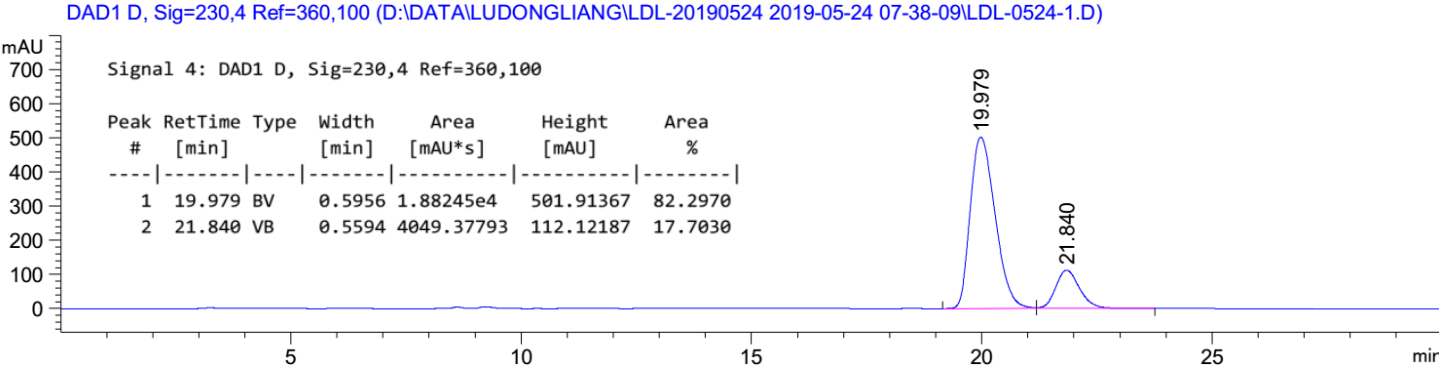




\section{References}

(1) Y.-H. Chen, D.-J. Cheng, J. Zhang, Y. Wang, X.-Y. Liu, B. Tan, J. Am. Chem. Soc. 2015, $137,15062-15065$.

(2) Y.-H. Chen, L.-W. Qi, F. Fang, B. Tan, Angew. Chem., Int. Ed. 2017, 56, 16308 -16312.

(3) L.-W. Qi, J.-H. Mao, J. Zhang, B. Tan, Nat. Chem. 2018, 10, 58-64.

(4) S. Billiet, K. D. Bruycker, F. Driessen, H. Goossens, V. V. Speybroeck, J. M. Winne, F. E. D Prez, Nat. Chem. 2014, 6, 815-821.

(5) J. J. Marugan, K. D. Haslow, C. Crysler, Bioorg. Med. Chem. Lett. 2004, 14, 4553-4555.

(6) S. T. Heller, E. E. Schultz, R. Sarpong, Angew. Chem., Int. Ed. 2012, 51, 8304-8308.

(7) S.-Y. Li, J.-W. Zhang, X.-L. Li, D.-J. Cheng, B. Tan, J. Am. Chem. Soc. 2016, 138, 
16561-16566. 
Copies of NMR spectra
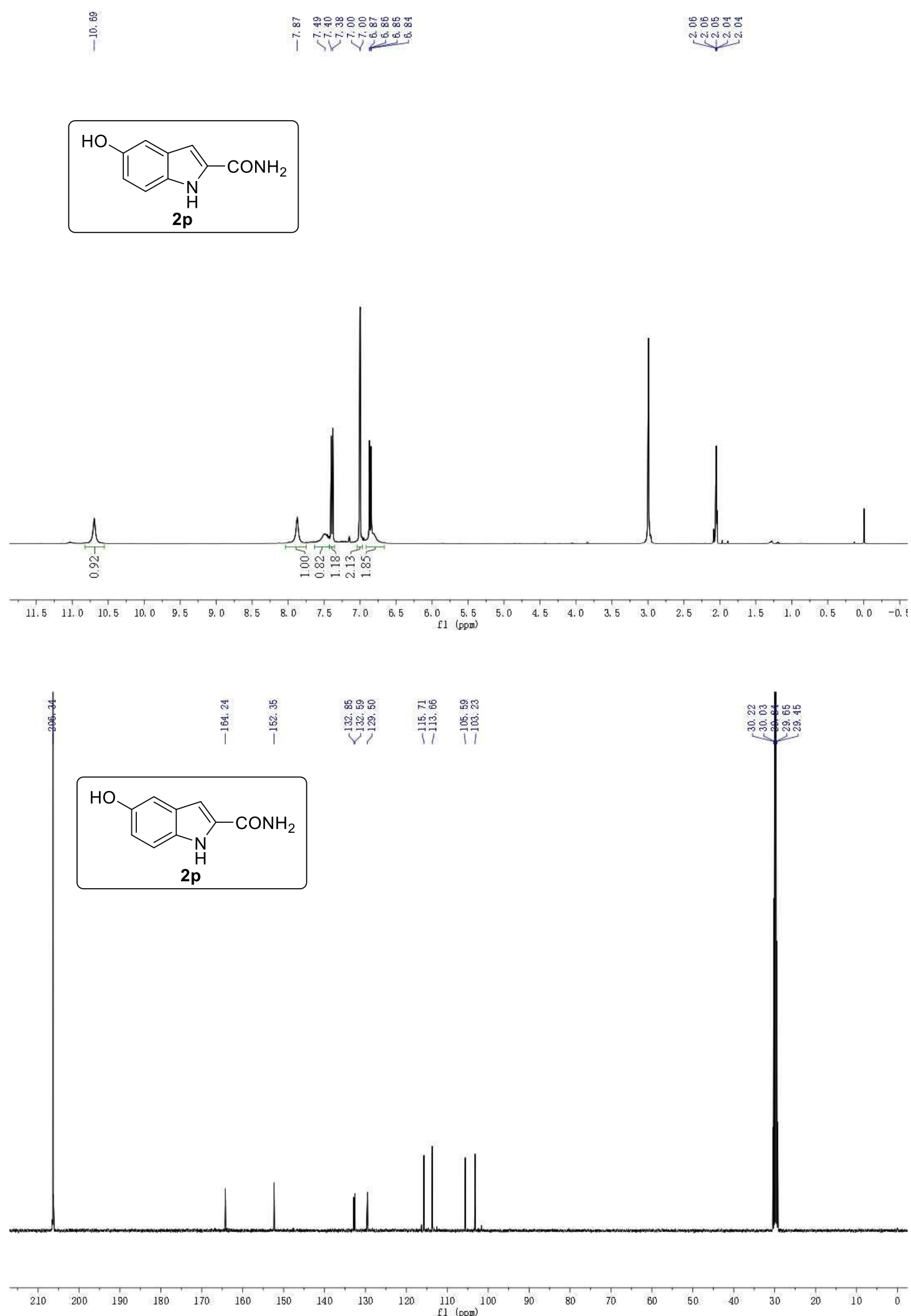

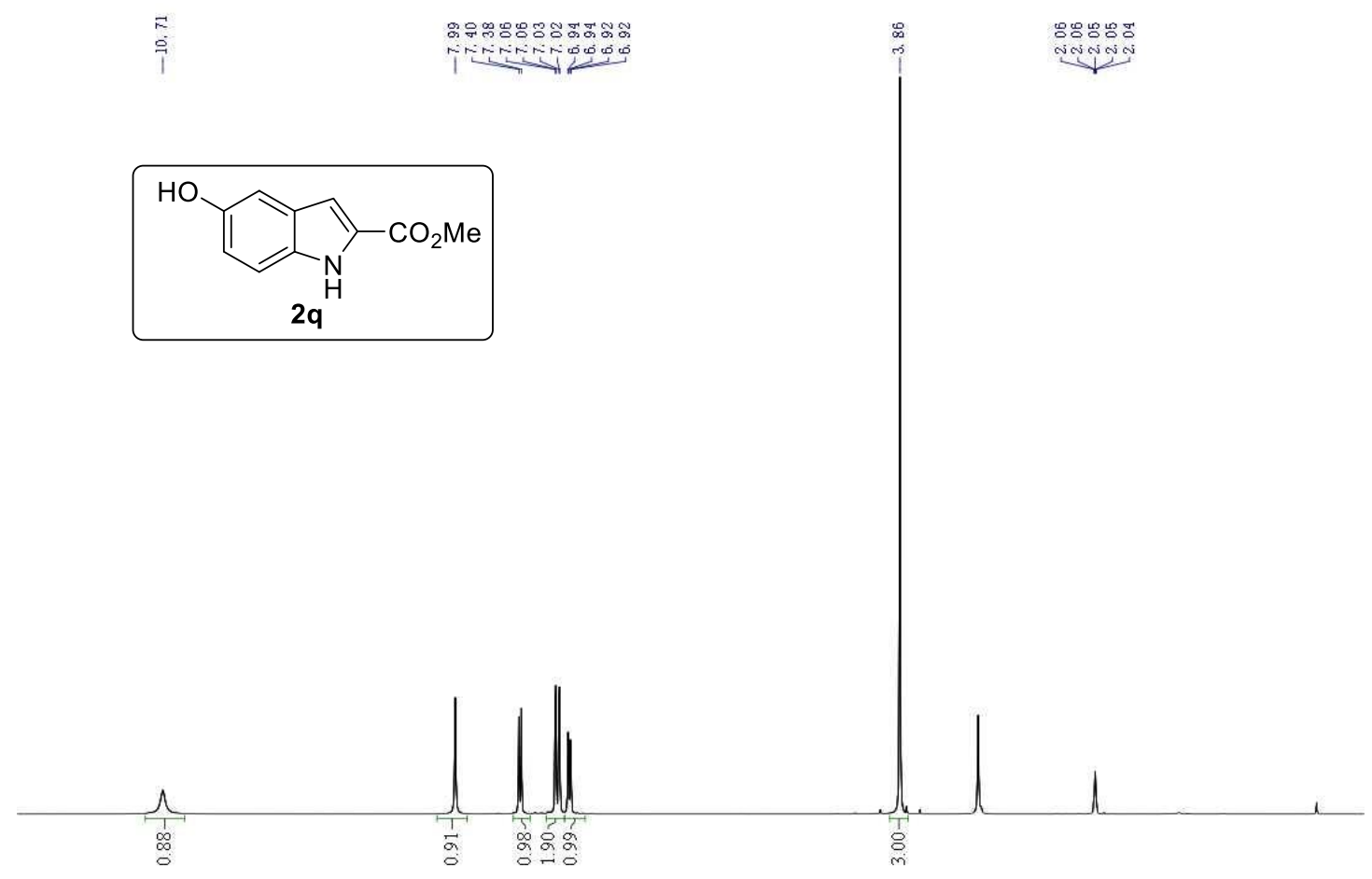
\begin{tabular}{llllllllllllllllllllllllllllllll}
\hline 2.0 & 11.5 & 11.0 & 10.5 & 10.0 & 9.5 & 9.0 & 8.5 & 8.0 & 7.5 & 7.0 & 6.5 & 6.0 & 5.5 & 5.0 & 4.5 & 4.0 & 3.5 & 3.0 & 2.5 & 2.0 & 1.5 & 1.0 & 0.5 & 0.0
\end{tabular}

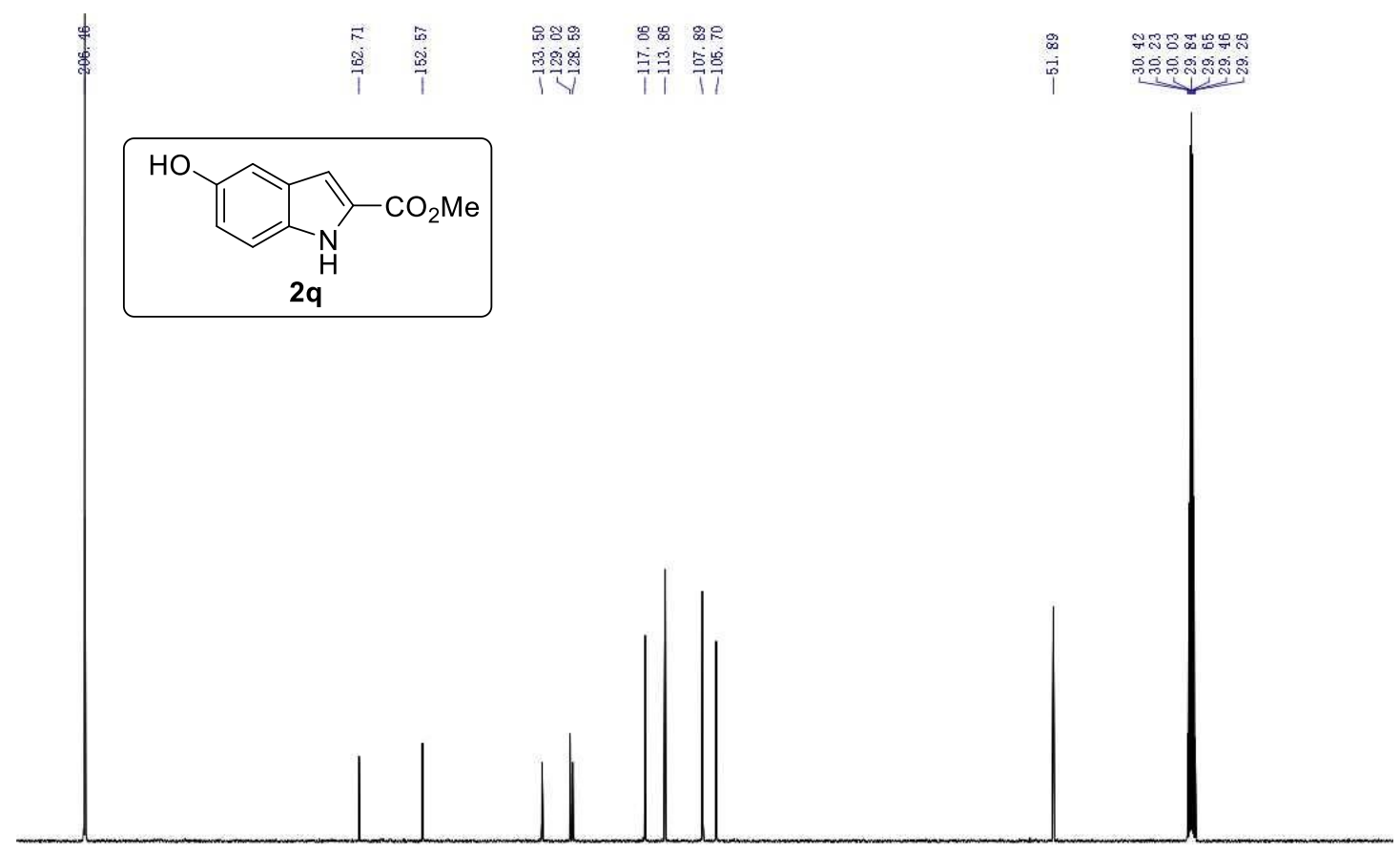

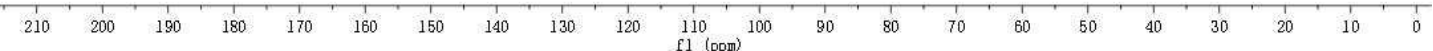




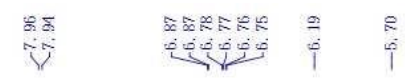

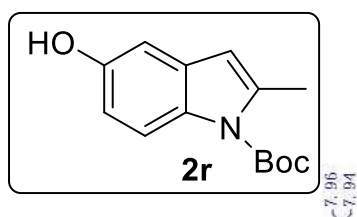

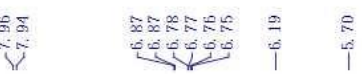

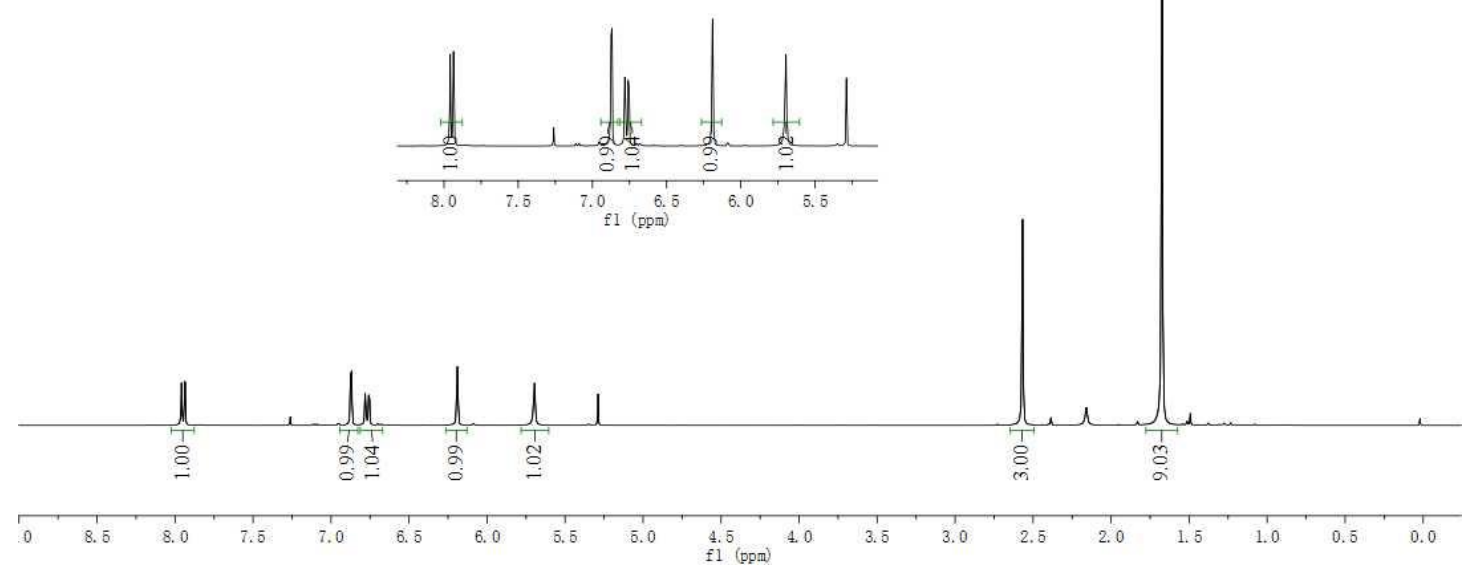

$\stackrel{0}{i} \quad i$

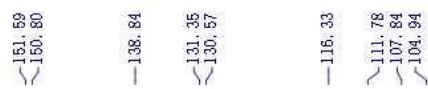

$\underset{1}{\infty}$

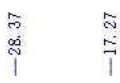
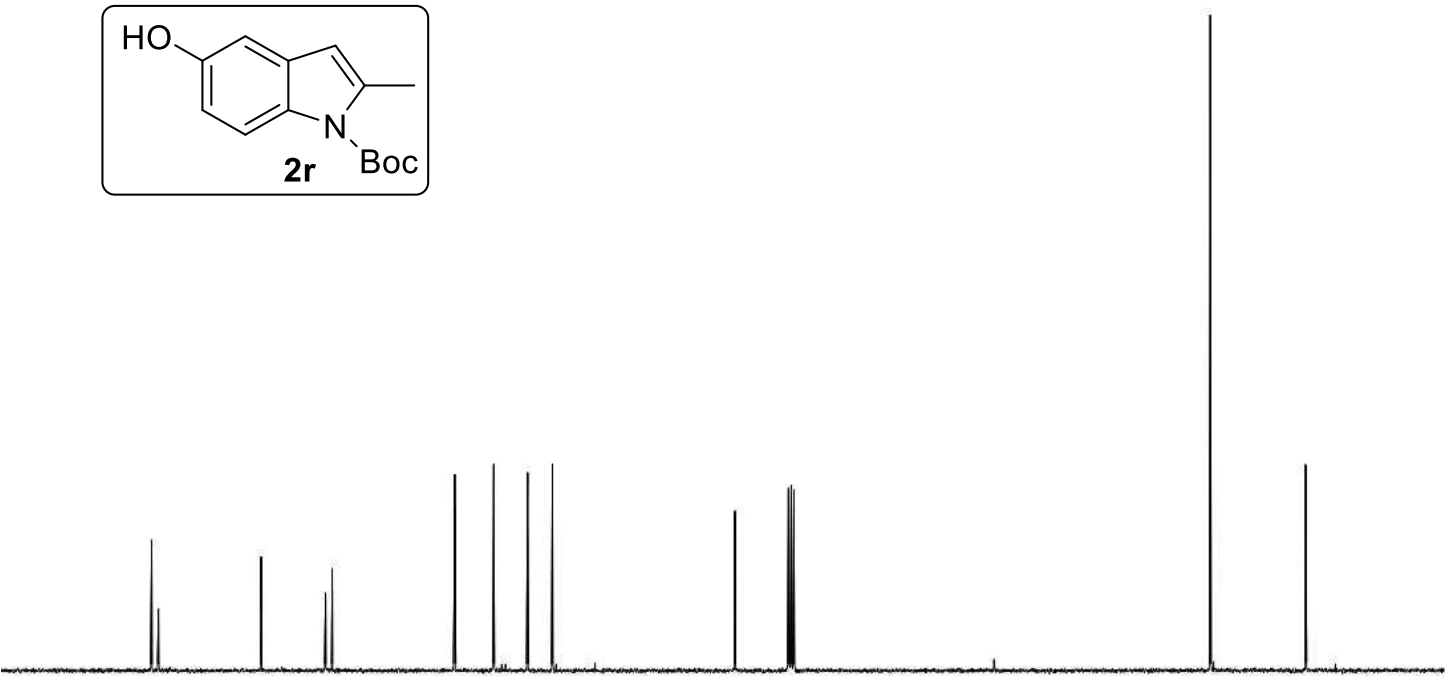

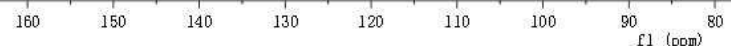


<smiles>CC(C)(C)[C@H]1Nc2ccccc2[C@H]1c1c(O)ccc(O)c1-c1ccccc1</smiles>

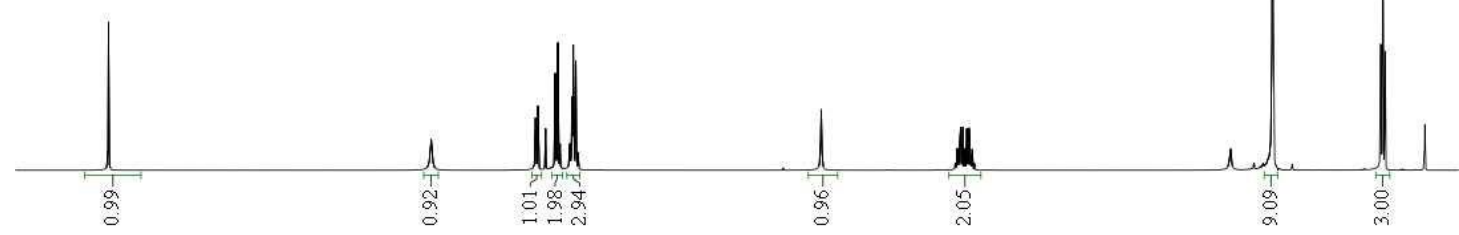

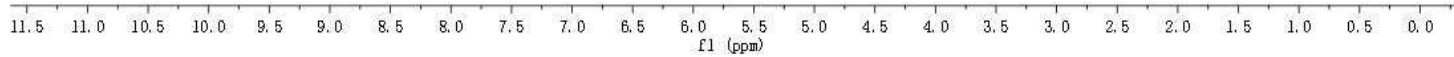

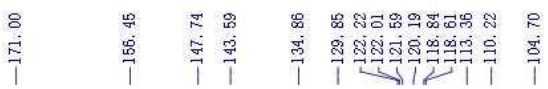

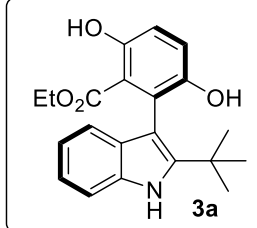

$\mathrm{H} \quad 3 a$
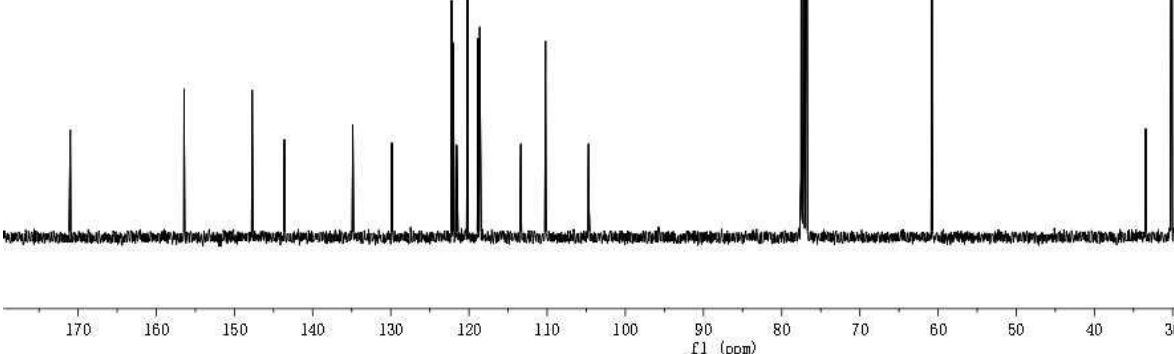

1010 

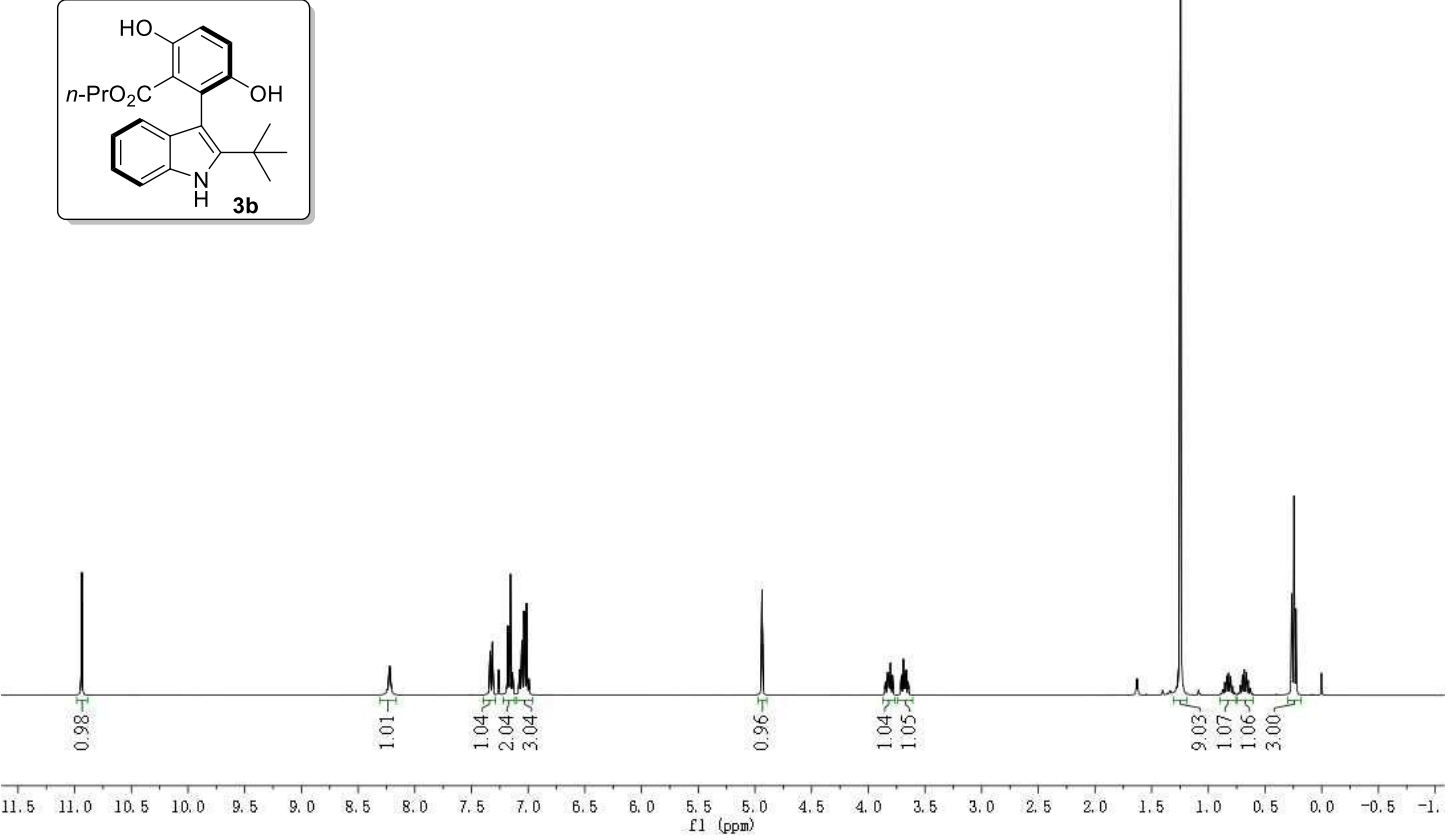

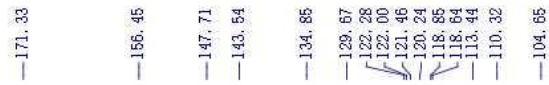

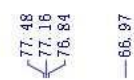

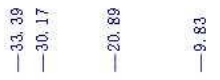<smiles>CC(C)(C)c1[nH]c2ccccc2c1-c1c(O)ccc(O)c1C(=O)O</smiles>

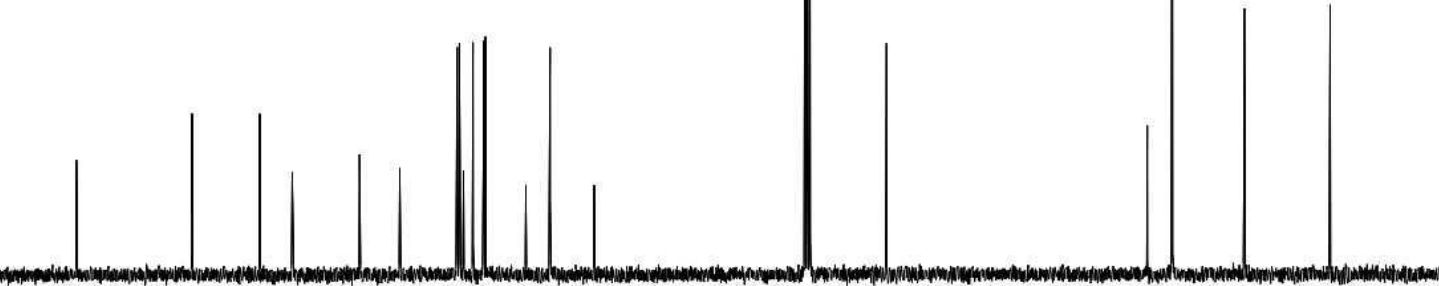

$\frac{1}{180}$

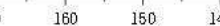

$130 \quad 120$

$\mathrm{fl}^{90}(\mathrm{ppm})$ 

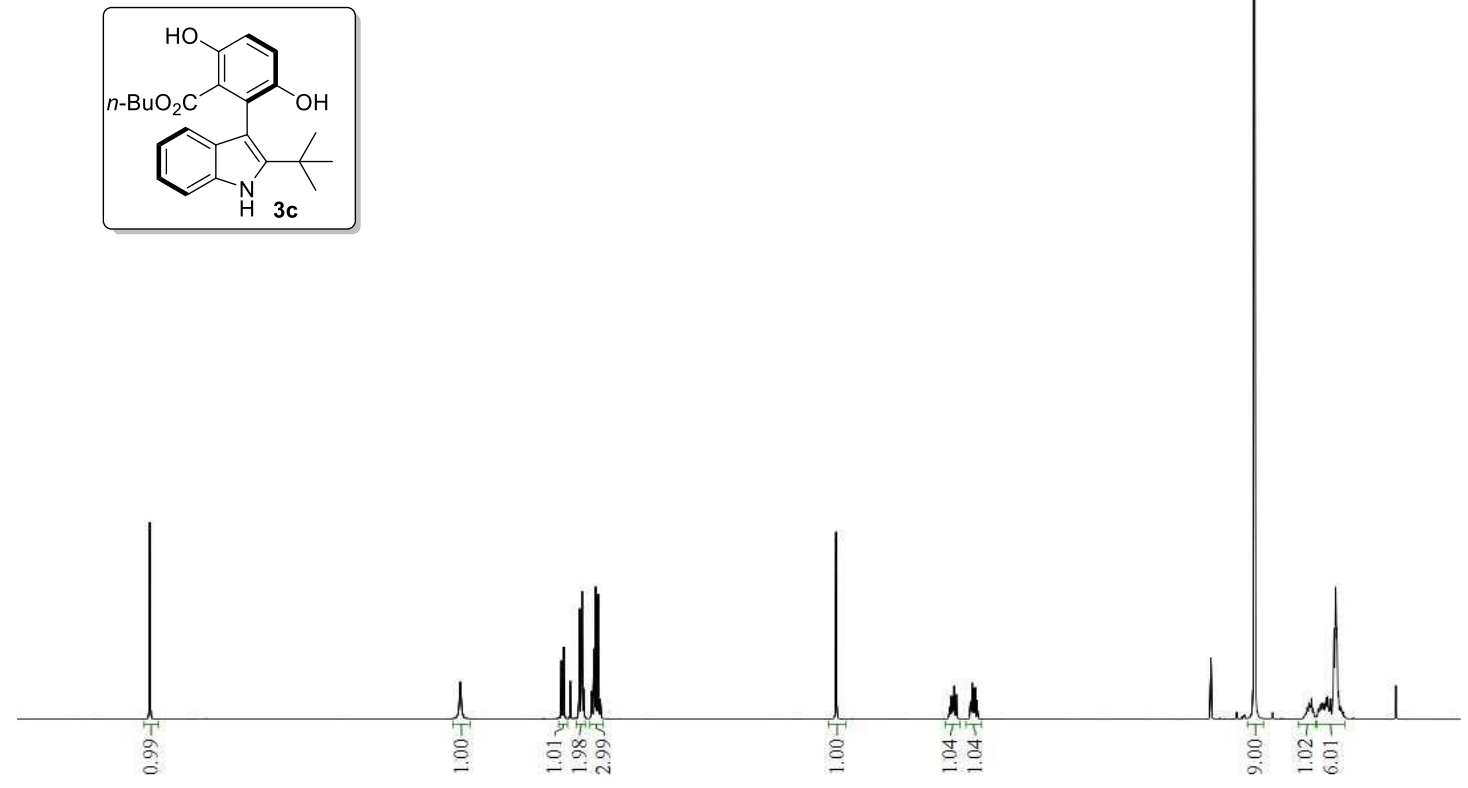

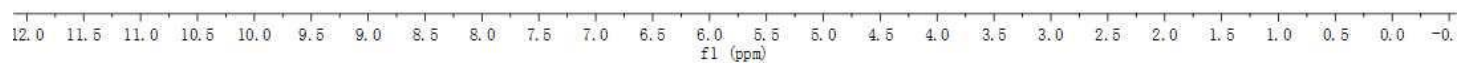

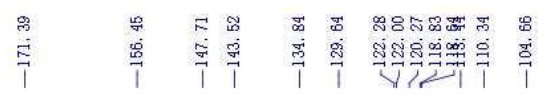

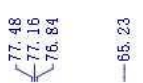

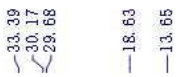

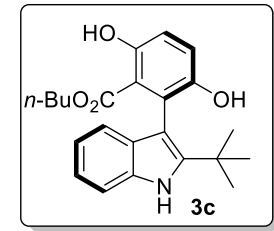

H $3 c$

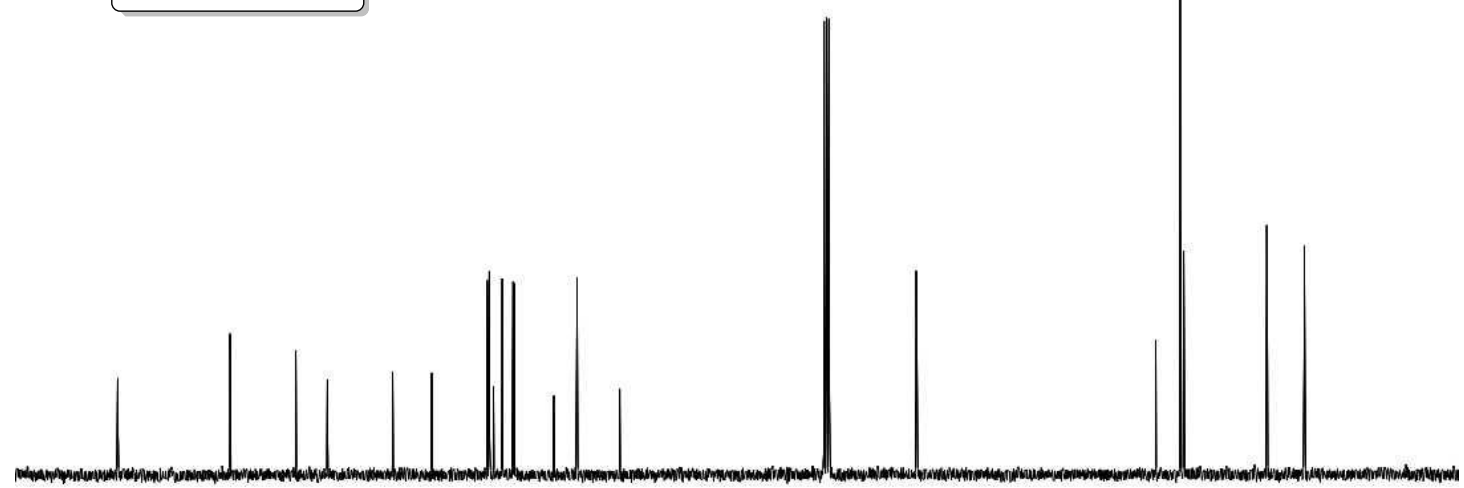




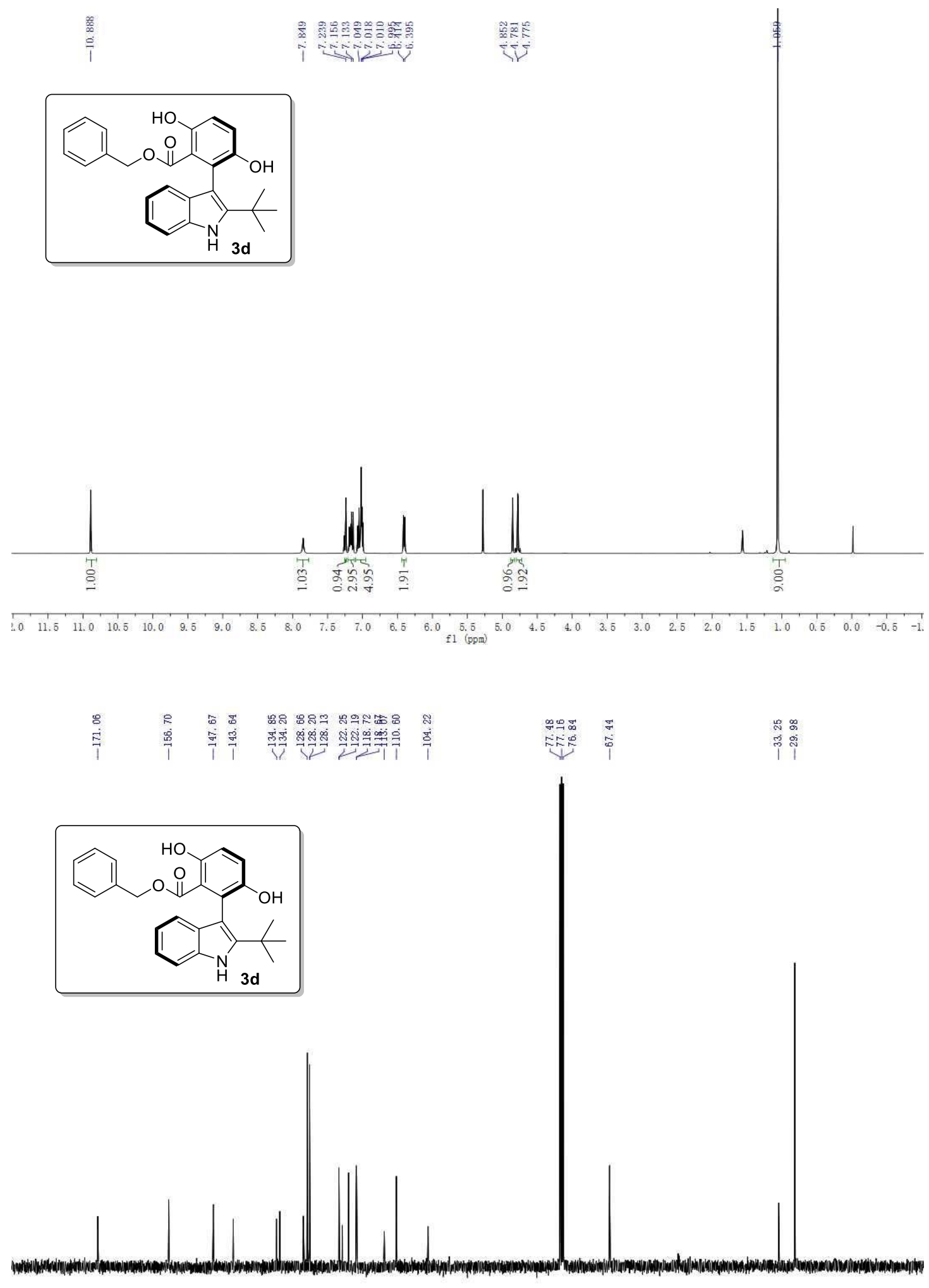

$\begin{array}{lllllllll}180 & 170 & 160 & 150 & 140 & 130 & 120 & 110 & 100 \\ \mathrm{f} 1(\mathrm{ppm}) & 10\end{array}$ 


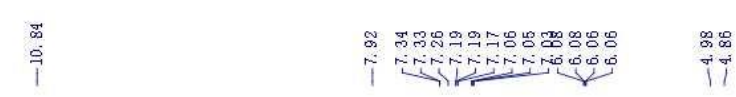

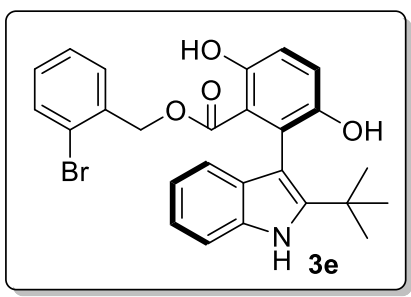

\section{i}


$\stackrel{1}{1}$

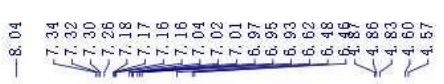
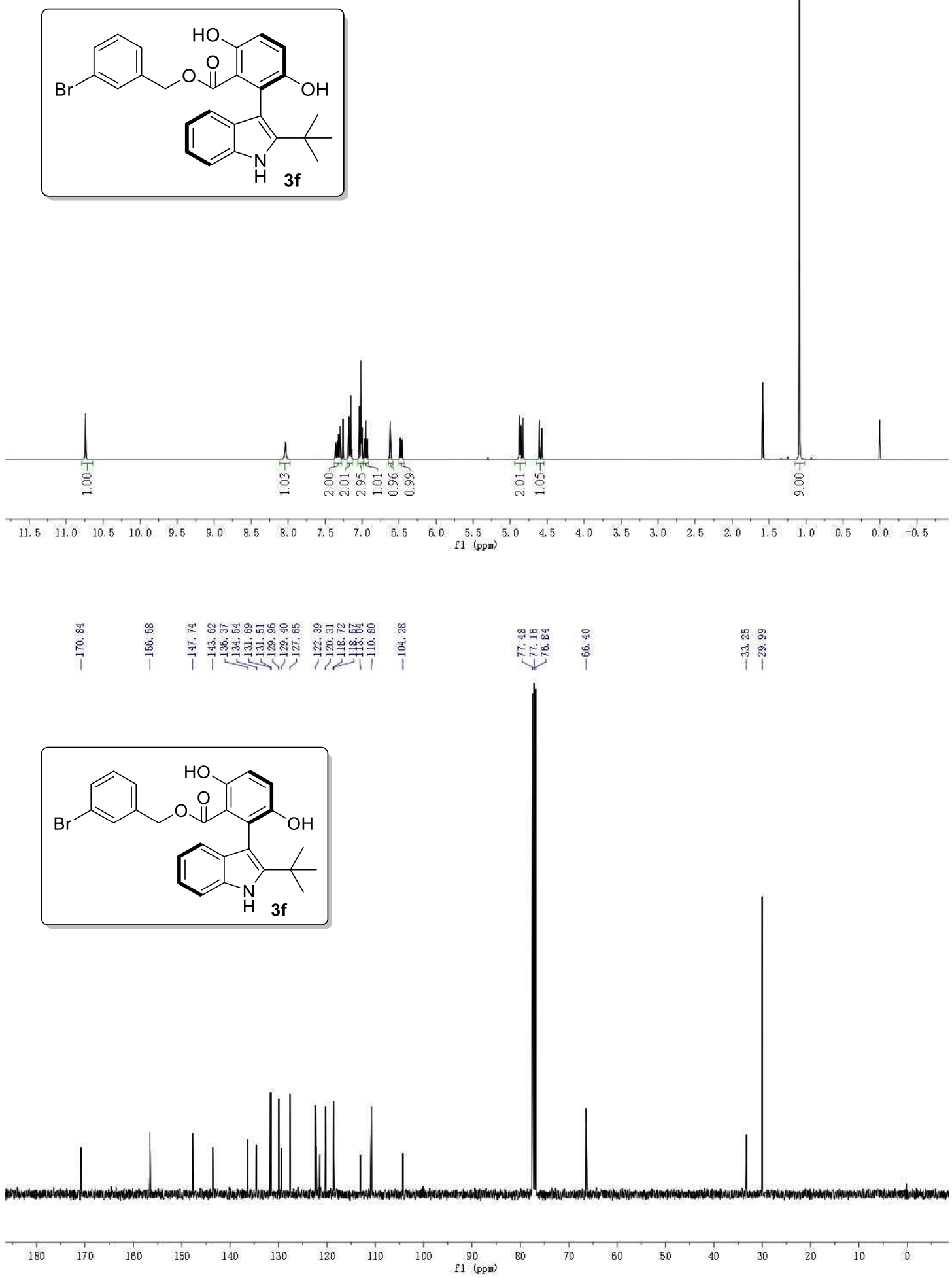

S67 


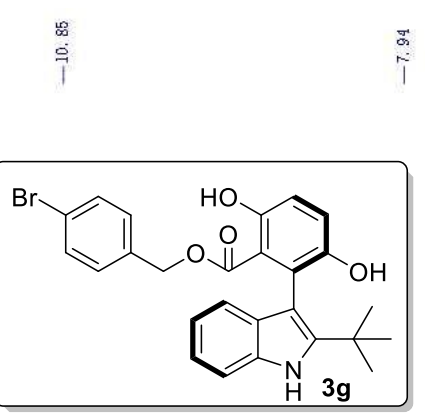

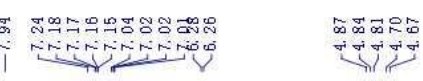

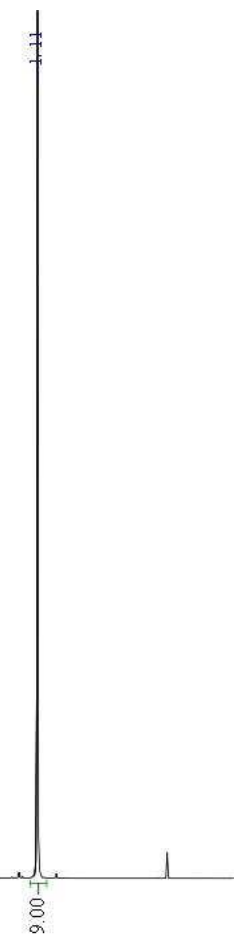

\begin{tabular}{lllllllllllllllllllllllllllllllllllll}
\hline 11.5 & 11.0 & 10.5 & 10.0 & 9.5 & 9.0 & 8.5 & 8.0 & 7.5 & 7.0 & 6.5 & 6.0 & 5.5 & 5.0 & 4.5 & 4.0 & 3.5 & 3.0 & 2.5 & 2.0 & 1.5 & 1.0 & 0.5 & 0.0 & -0.0
\end{tabular}

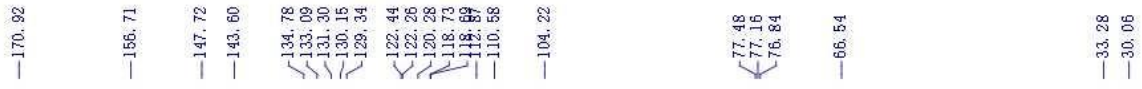
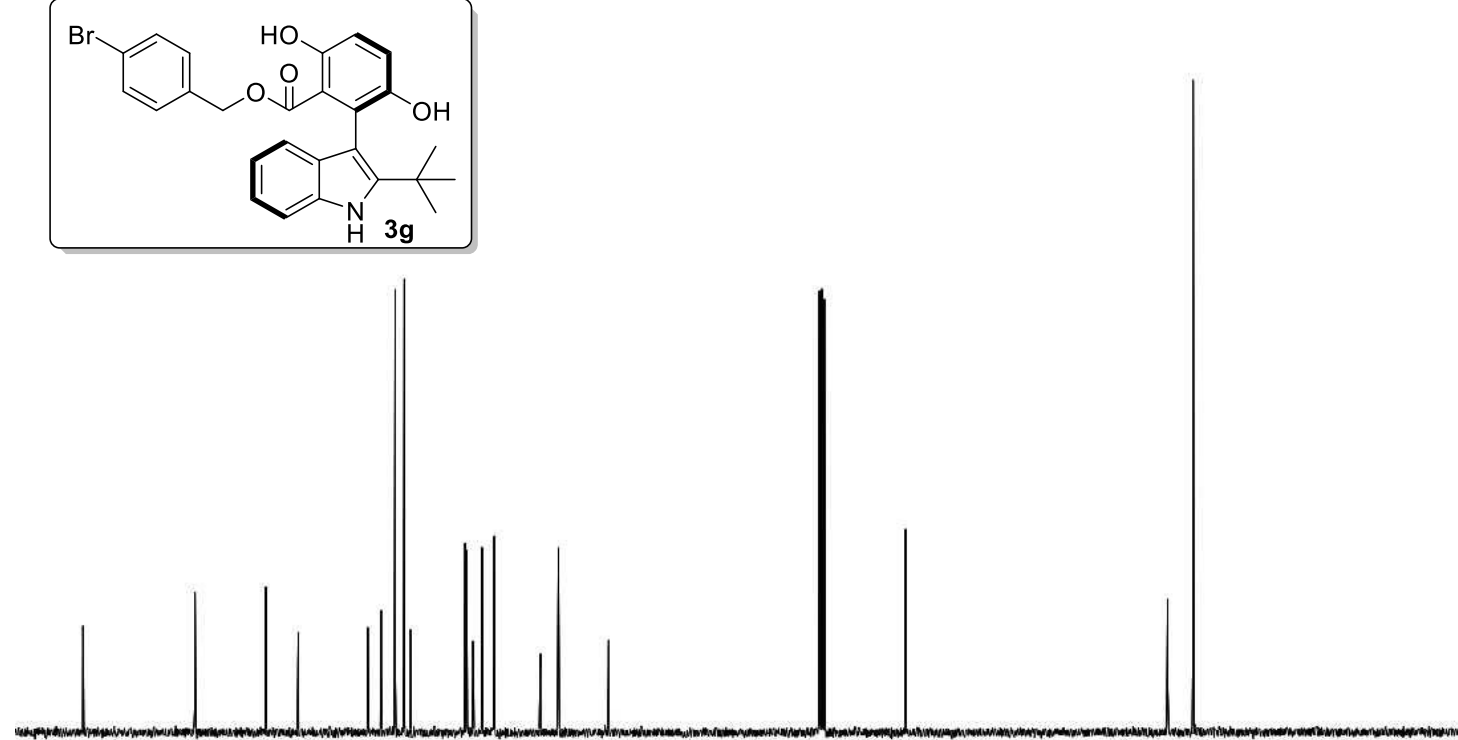

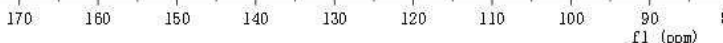



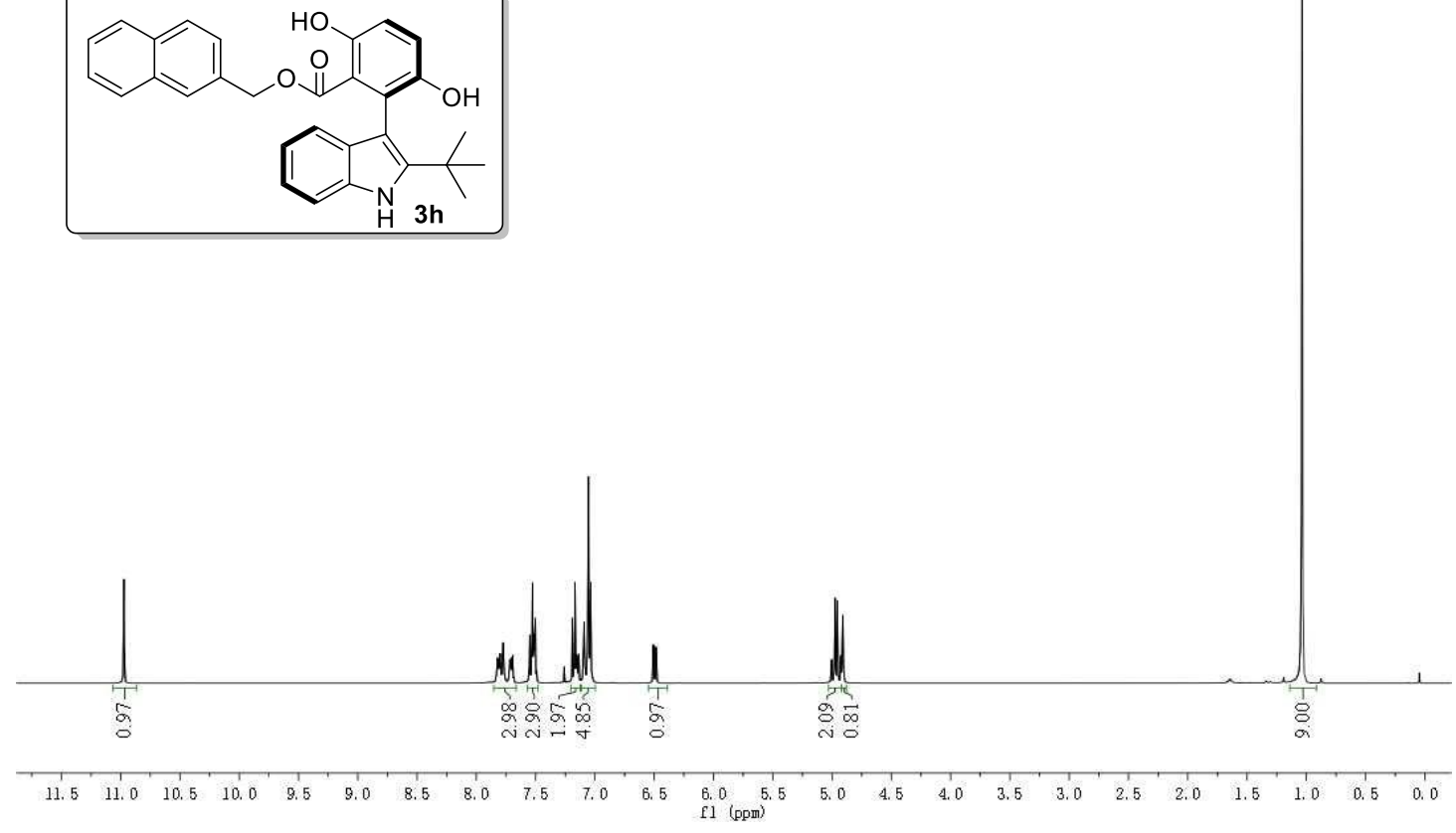

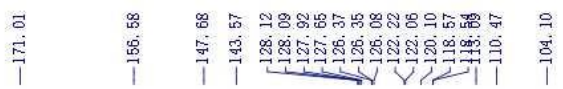

i
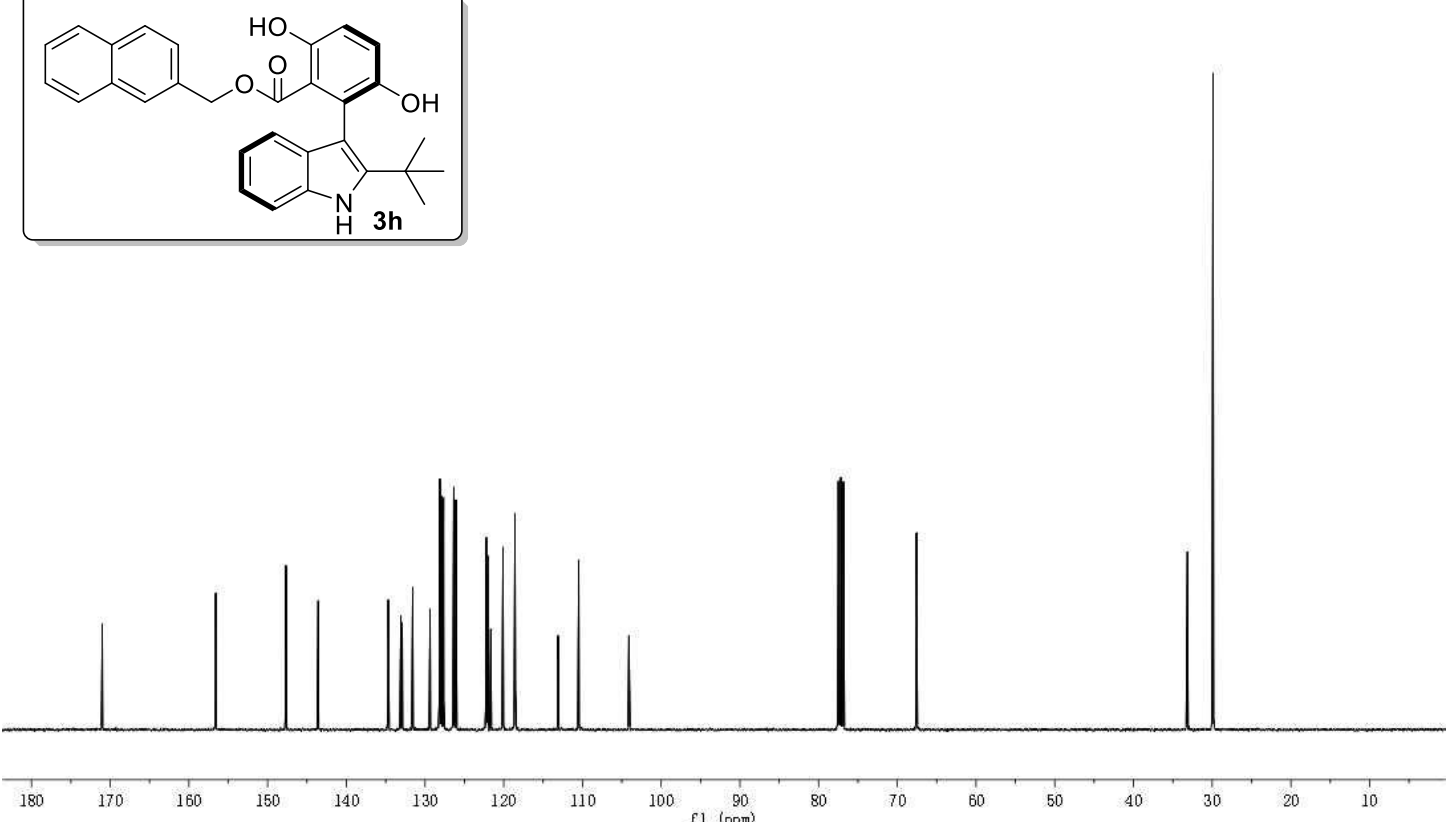


\section{$\underset{\sim}{\sim}$}

추요
ํㅜㅇ<smiles></smiles>

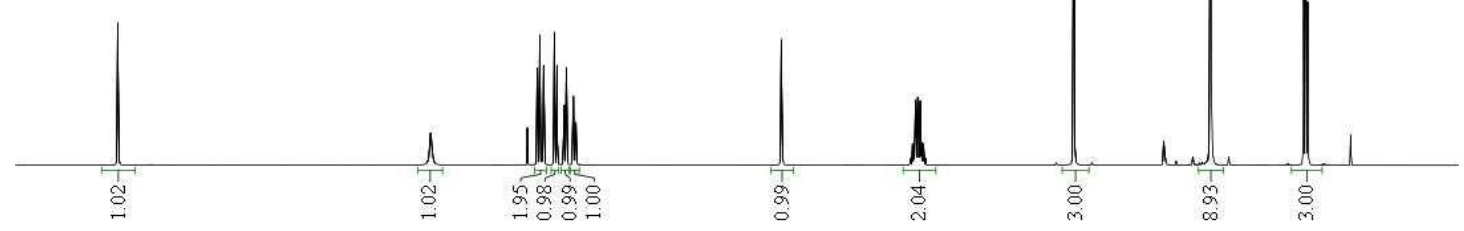

$\begin{array}{rlllllllllllllllllllllllll}11.5 & 11.0 & 10.5 & 10.0 & 9.5 & 9.0 & 8.5 & 8.0 & 7.5 & 7.0 & 6.5 & 6.0 & 5.5 & 5.0 & 4.5 & 4.0 & 3.5 & 3.0 & 2.5 & 2.0 & 1.5 & 1.0 & 0.5 & 0.0 & -0.5\end{array}$

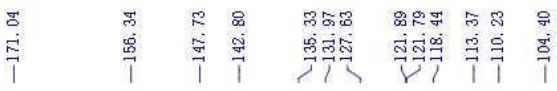

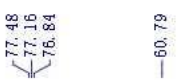

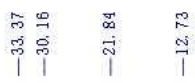
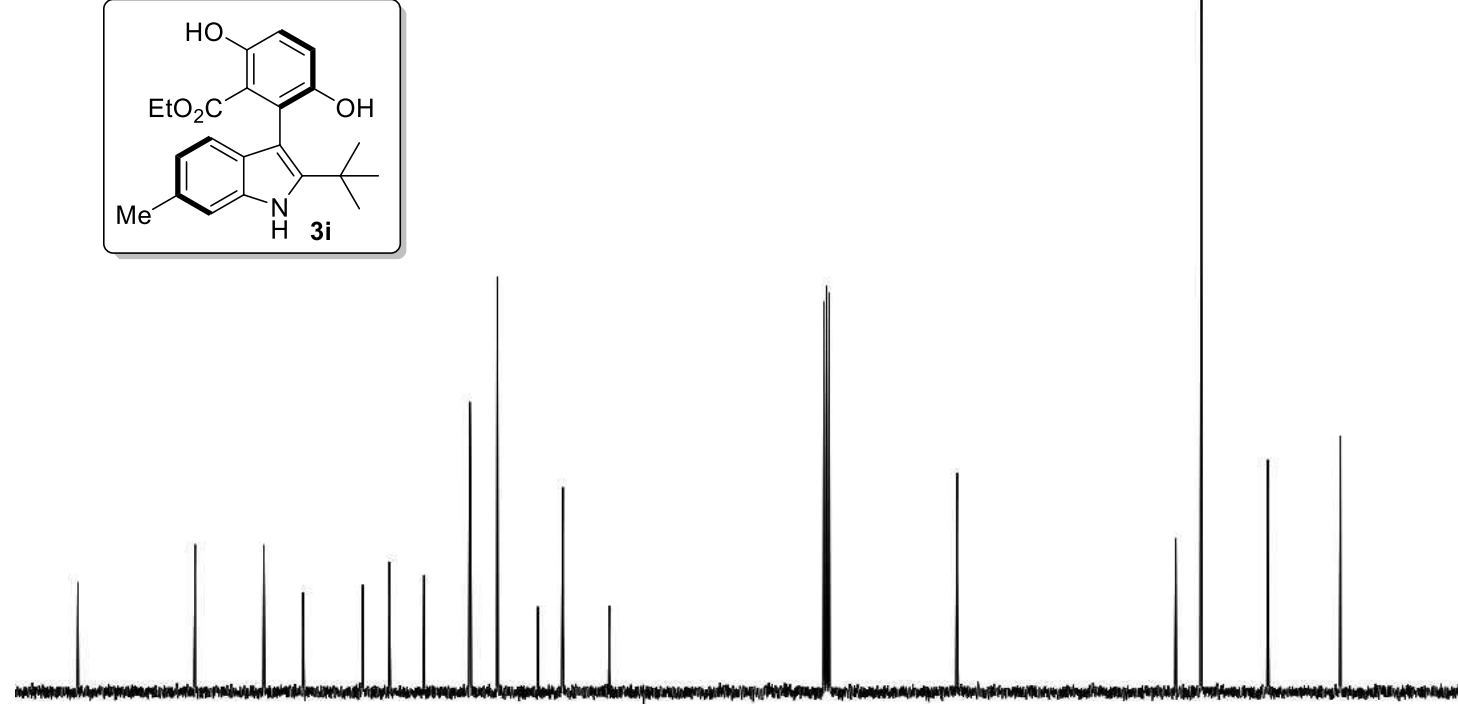

$170 \quad 160$

150

$100 \quad 90$
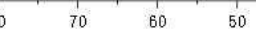

40

10 

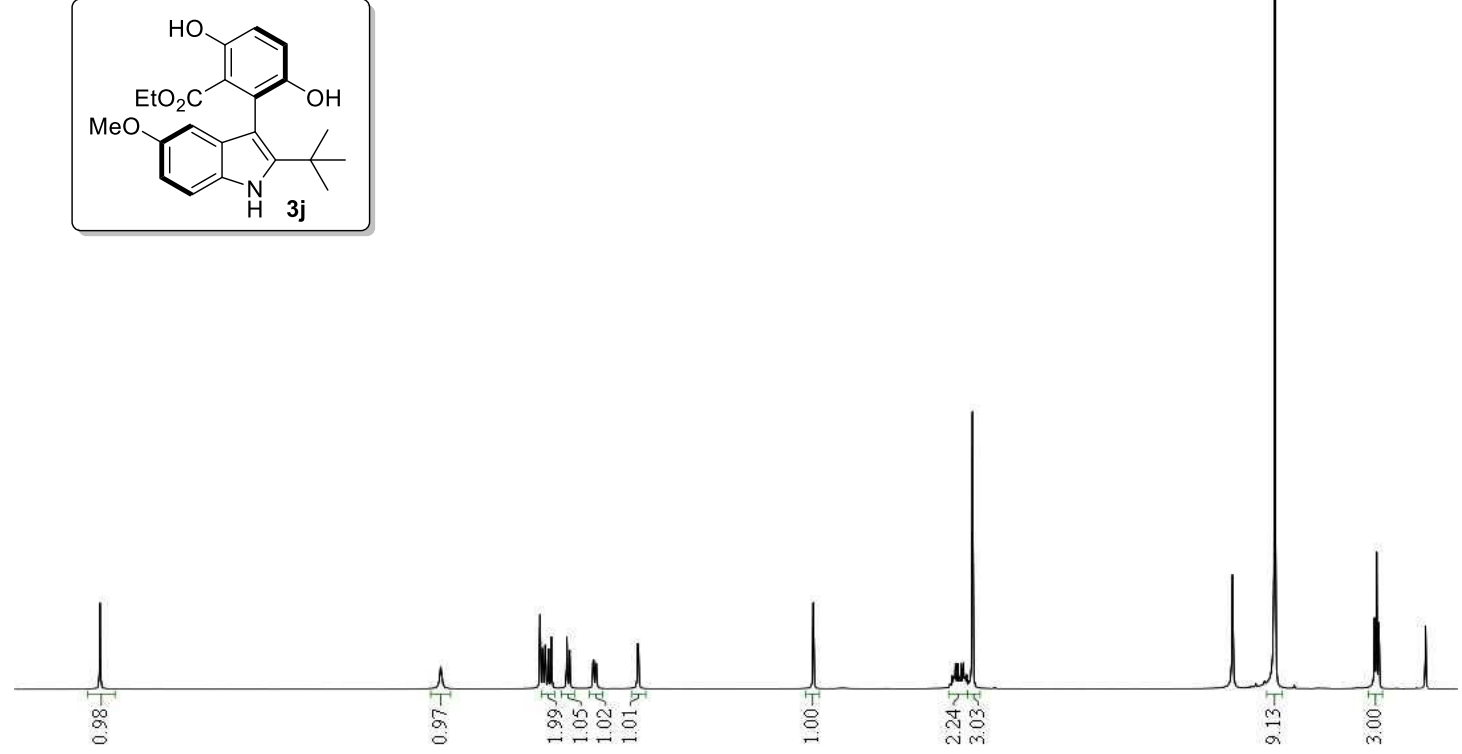

$\begin{array}{rrrrrrrrrrrrrrrrrrrrrrrrrr}1.5 & 11.0 & 10.5 & 10.0 & 9.5 & 9.0 & 8.5 & 8.0 & 7.5 & 7.0 & 6.5 & 6.0 & 5.5 & 5.0 & 4.5 & 4.0 & 3.5 & 3.0 & 2.5 & 2.0 & 1.5 & 1.0 & 0.5 & 0.0\end{array}$
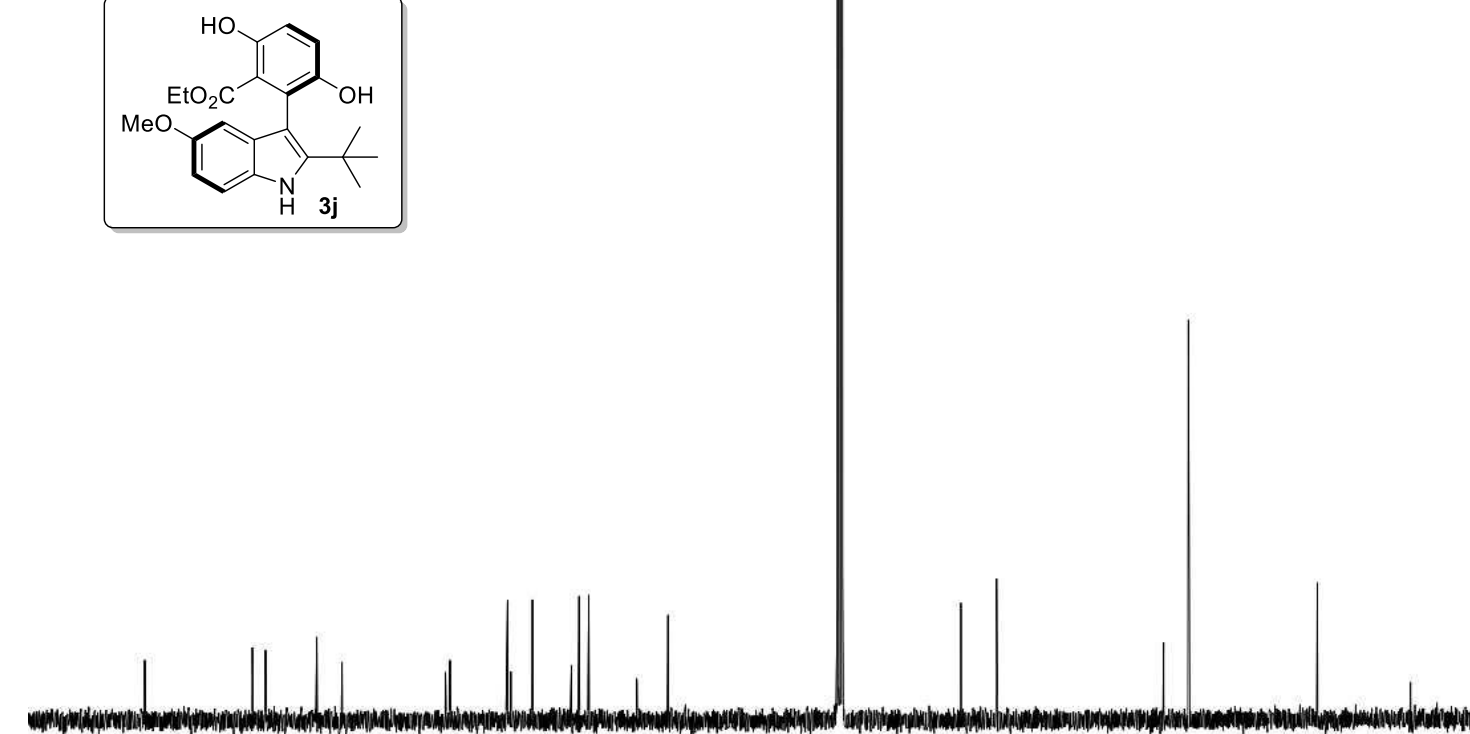

$180 \quad 170 \quad 160$

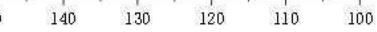
90 


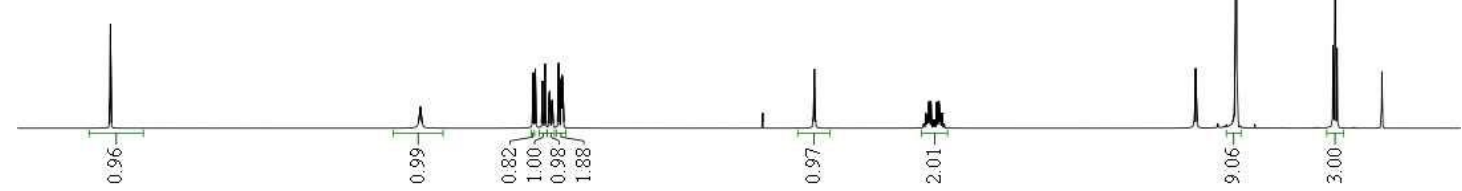
$\begin{array}{llllllllllllllllllllllllllllllllll}11.5 & 11.0 & 10.5 & 10.0 & 9.5 & 9.0 & 8.5 & 8.0 & 7.5 & 7.0 & 6.5 & 6.0 & 5.5 & 5.0 & 4.5 & 4.0 & 3.5 & 3.0 & 2.5 & 2.0 & 1.5 & 1.0 & 0.5 & 0.0 & -0.5\end{array}$

$$
\text { (1) }
$$

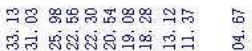

$\infty$
$\infty$
$\infty$
$\infty$
0

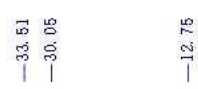

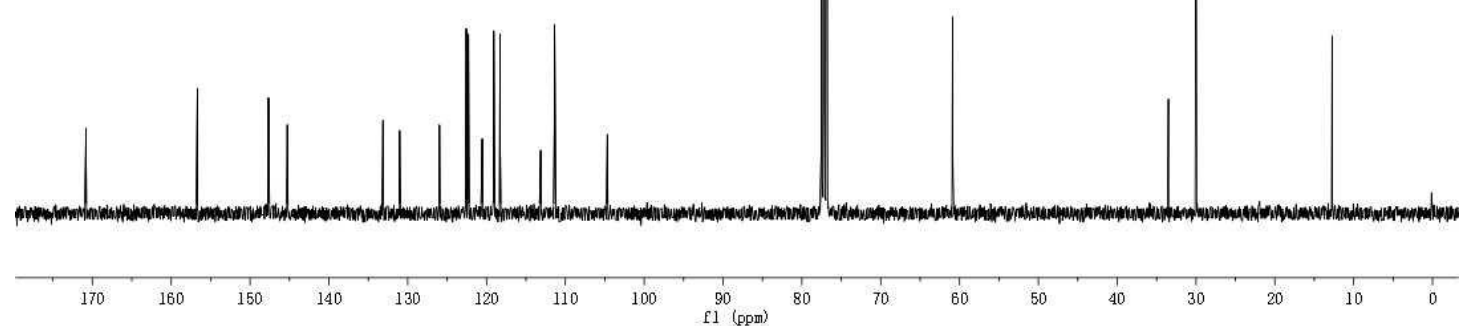



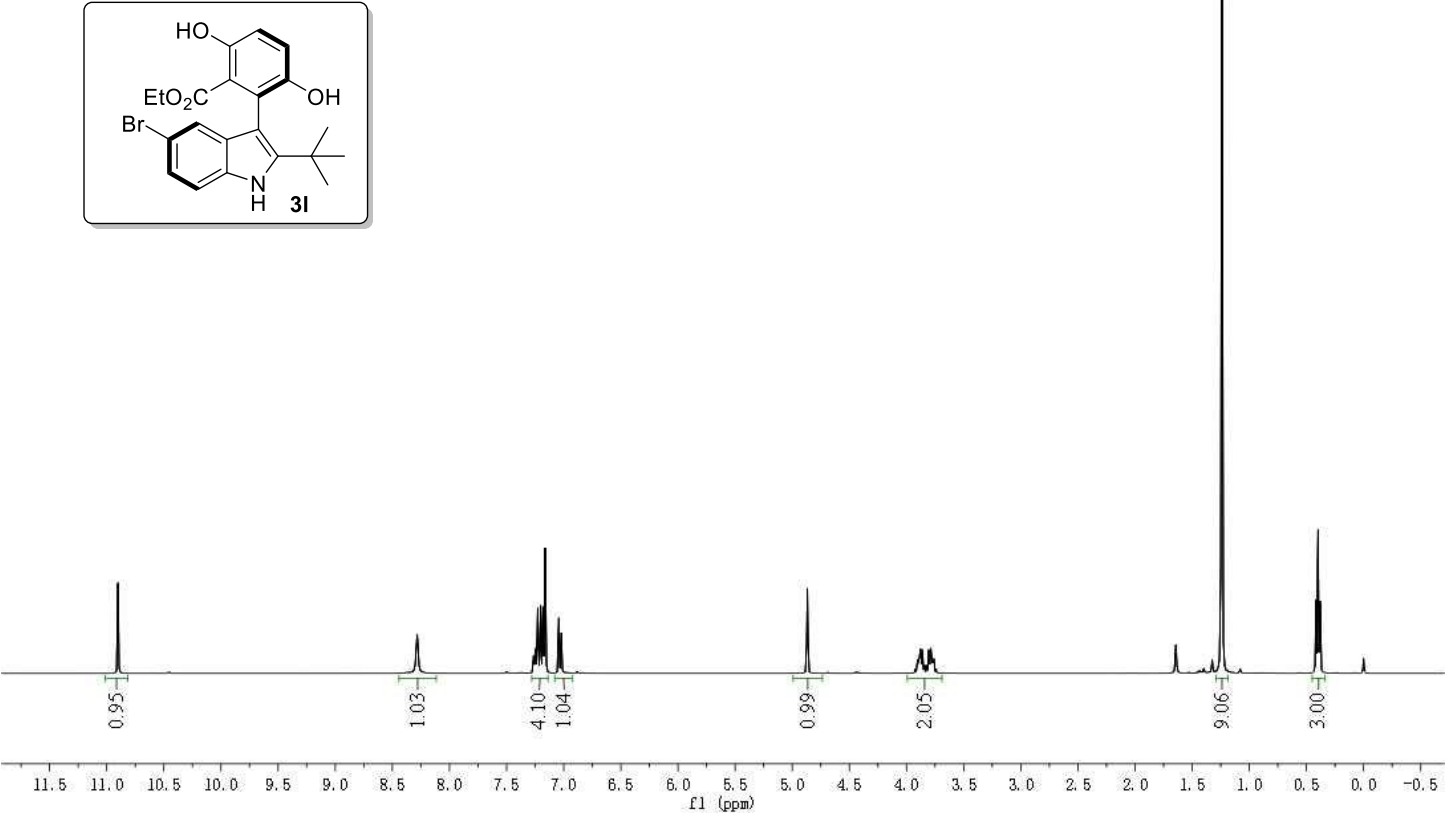

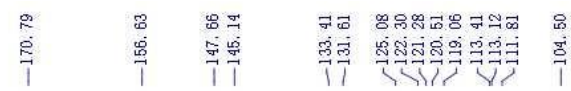

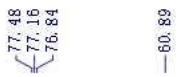

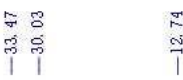
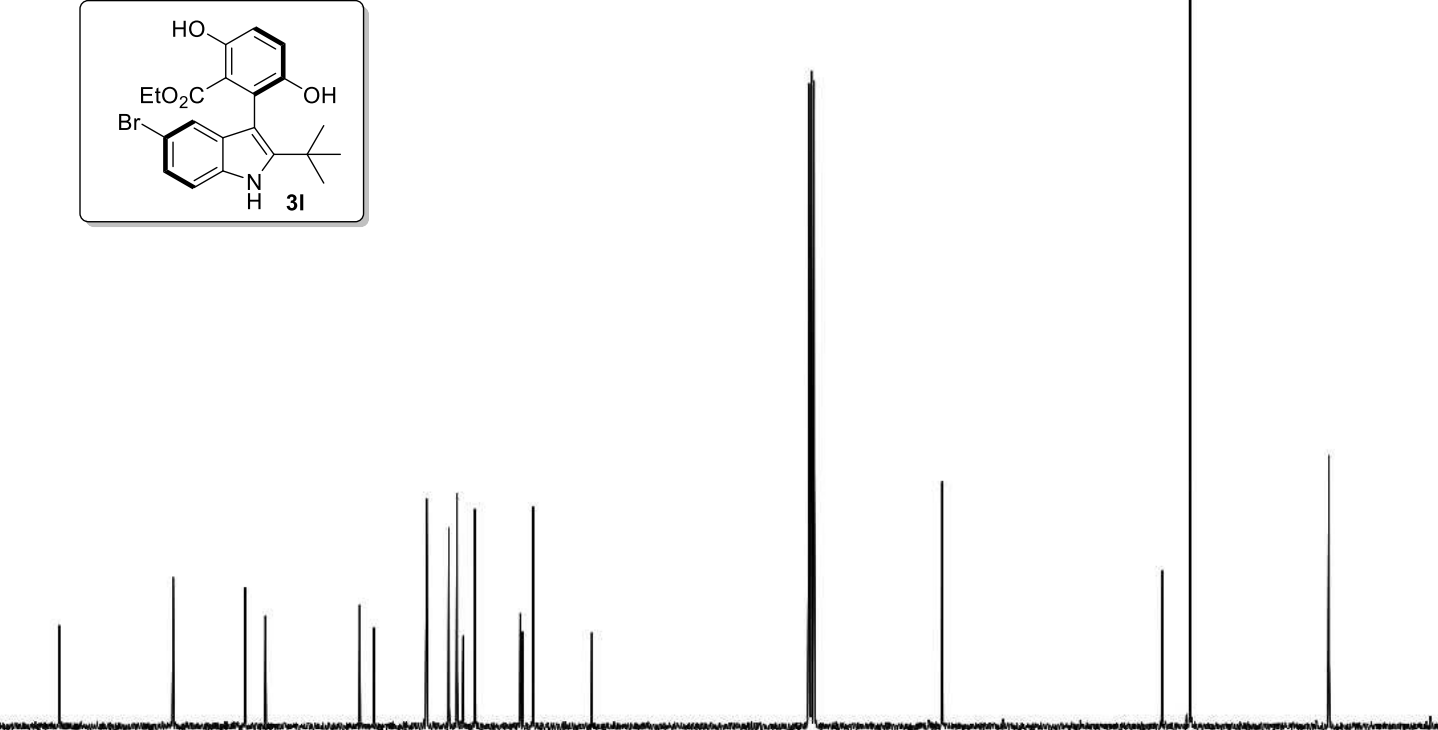

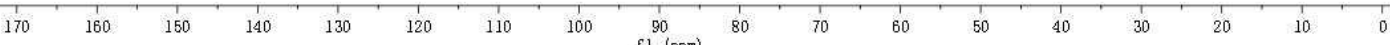



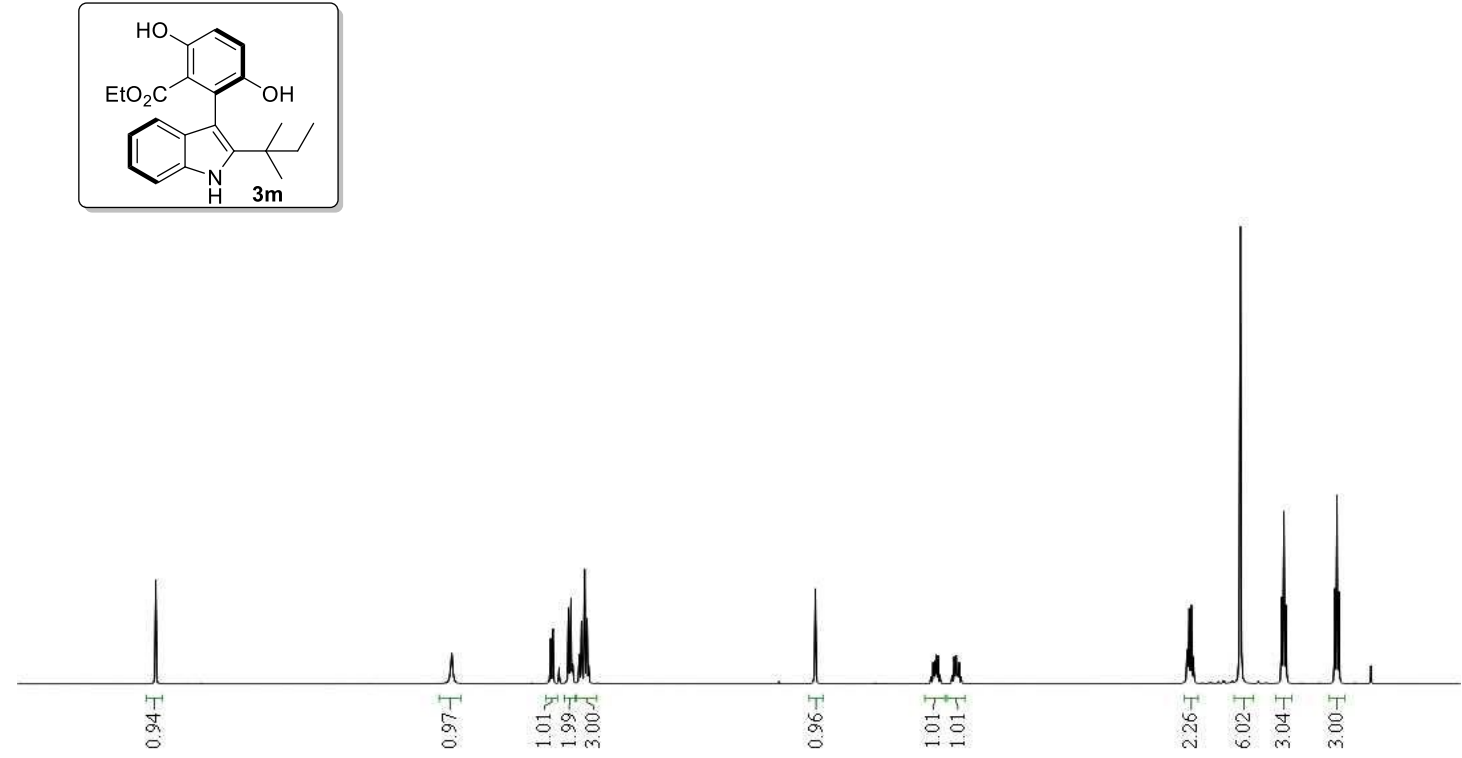

$\begin{array}{llllllllllllllllllllllllllllllllllllll}2.0 & 11.5 & 11.0 & 10.5 & 10.0 & 9.5 & 9.0 & 8.5 & 8.0 & 7.5 & 7.0 & 6.5 & 6.0 & 5.5 & 5.0 & 4.5 & 4.0 & 3.5 & 3.0 & 2.5 & 2.0 & 1.5 & 1.0 & 0.5 & 0.0 & -0.5\end{array}$

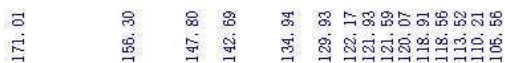

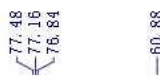

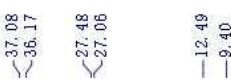<smiles>CCOC(=O)c1c(O)ccc(O)c1-c1c(C(C)(C)CC)[nH]c2ccccc12</smiles>

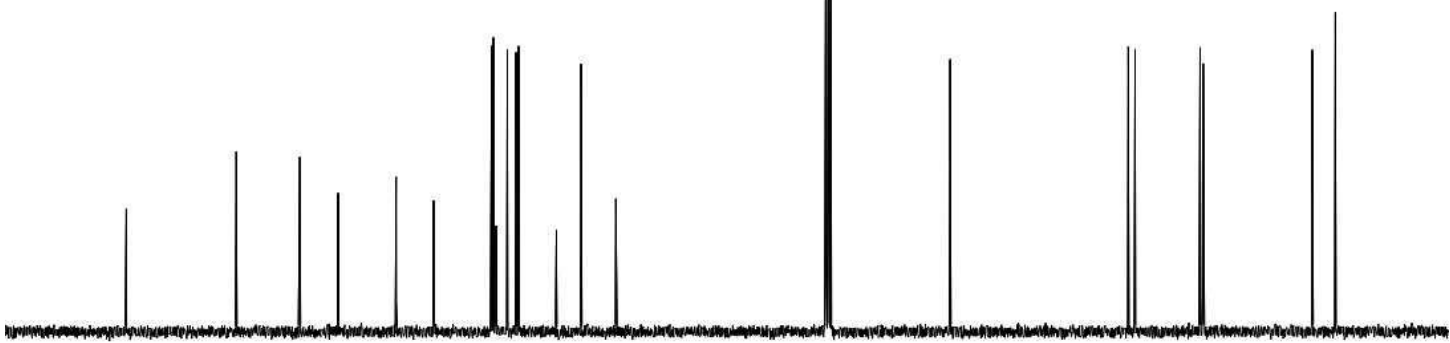

$\begin{array}{llllllllll}180 & 170 & 160 & 150 & 140 & 130 & 120 & 110 & 100 & 90\end{array}$ 

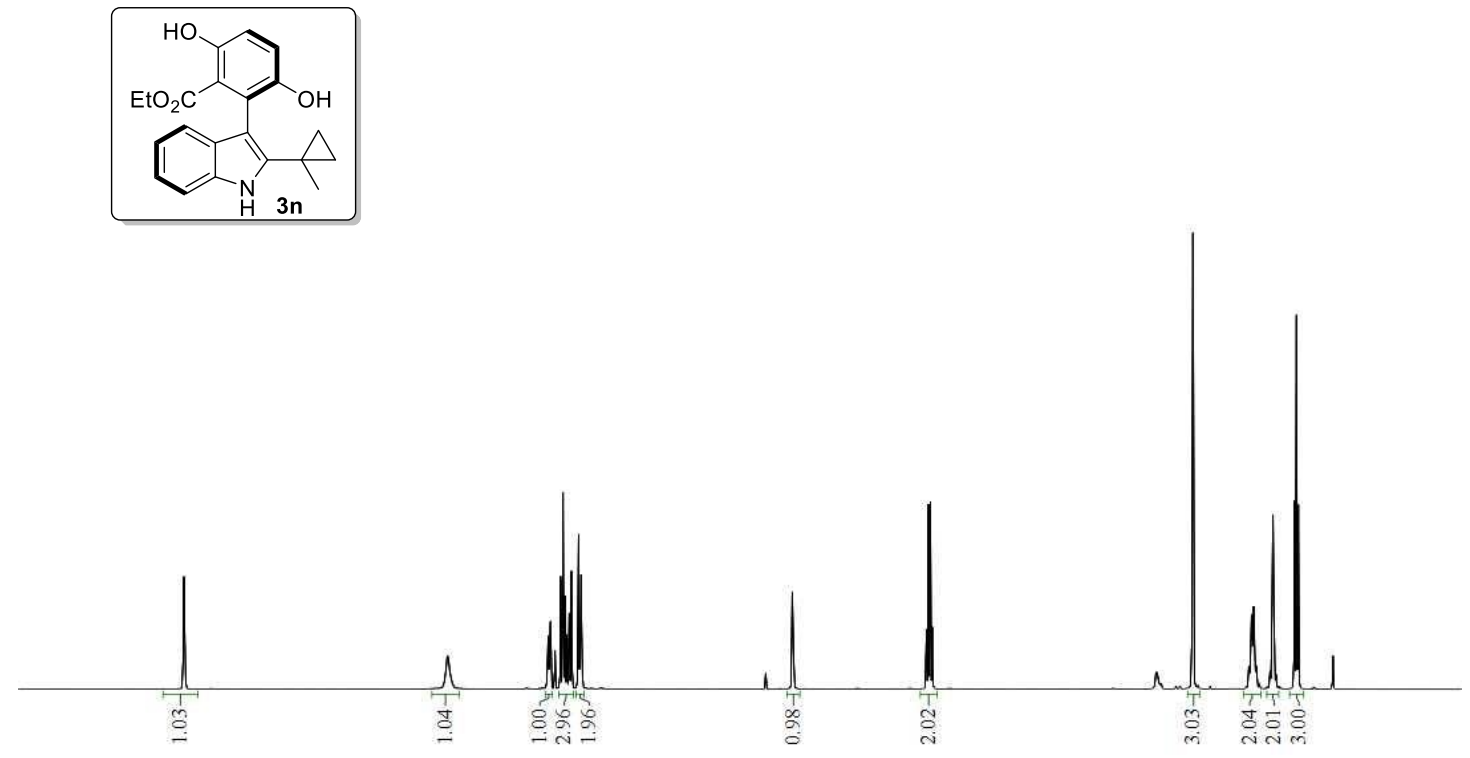

\begin{tabular}{rlllllllllllllllllllllllllllllllllll}
\hline 12.0 & 11.5 & 11.0 & 10.5 & 10.0 & 9.5 & 9.0 & 8.5 & 8.0 & 7.5 & 7.0 & 6.5 & 6.0 & 5.5 & 5.0 & 4.5 & 4.0 & 3.5 & 3.0 & 2.5 & 2.0 & 1.5 & 1.0 & 0.5 & 0.0 & -0.5 & -1.0
\end{tabular}

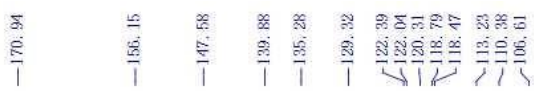

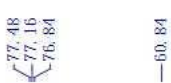

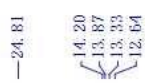
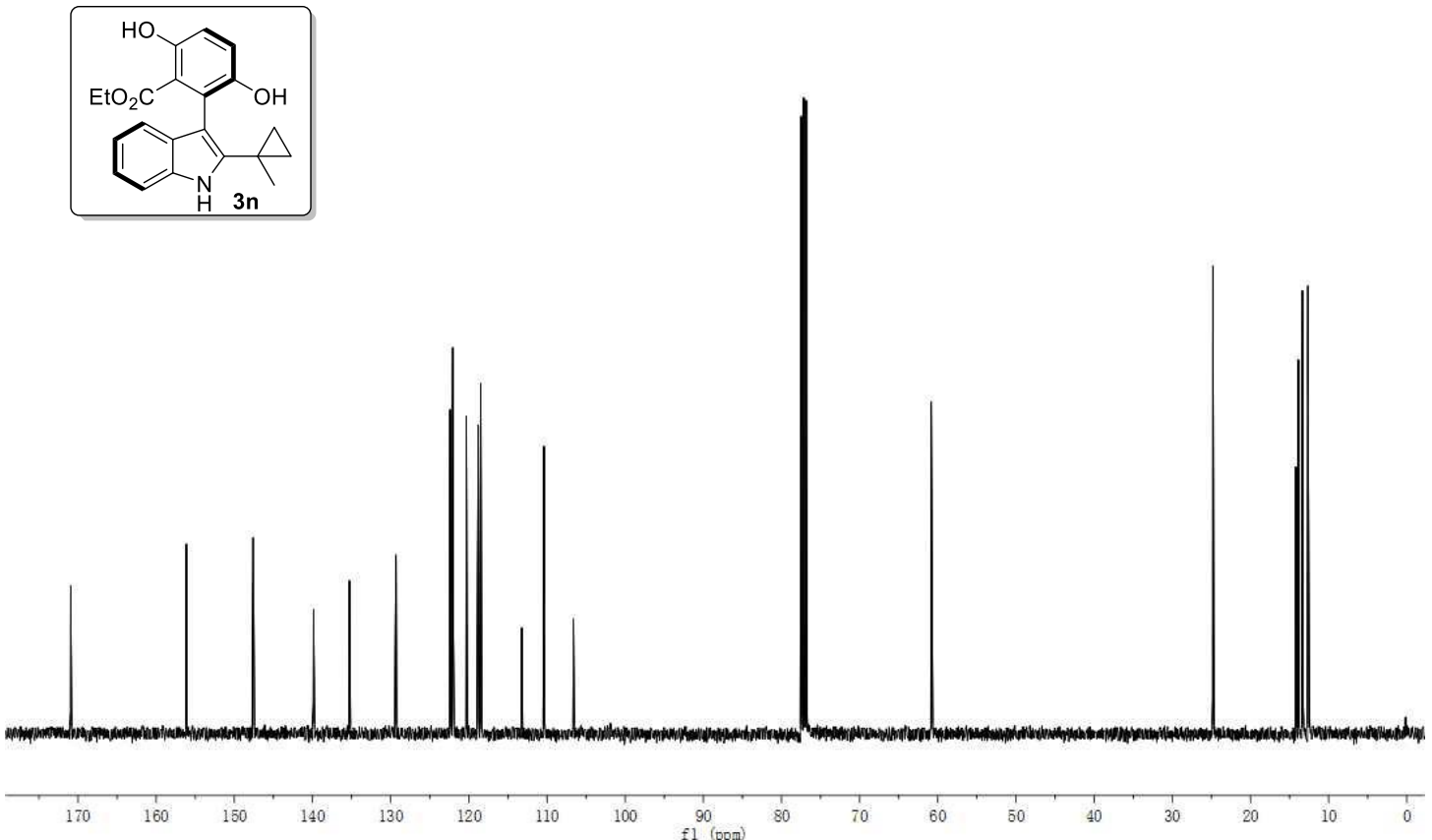

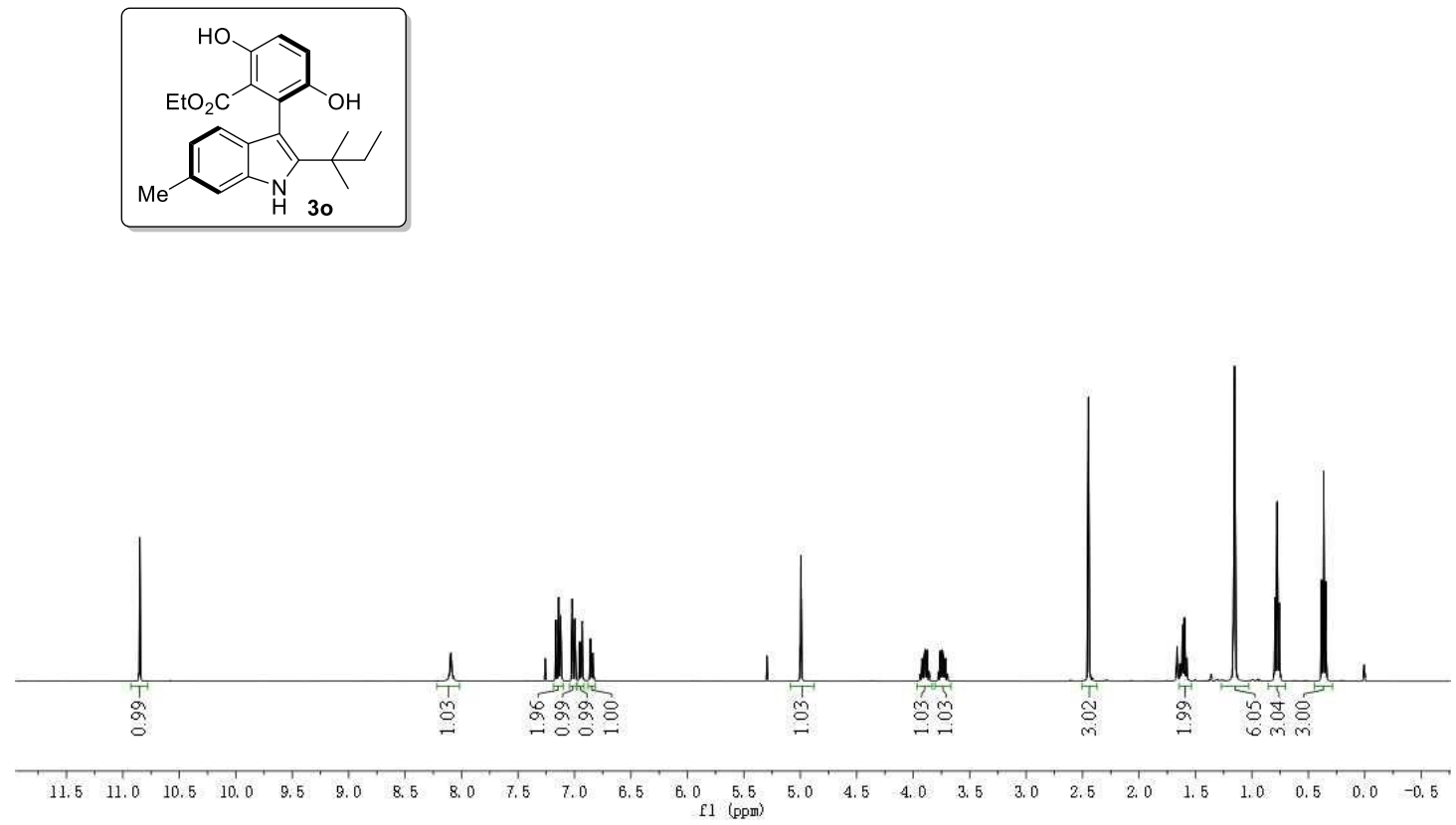

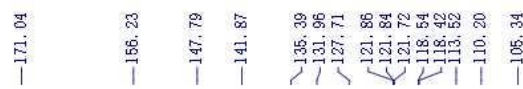

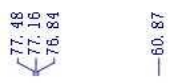

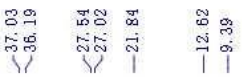
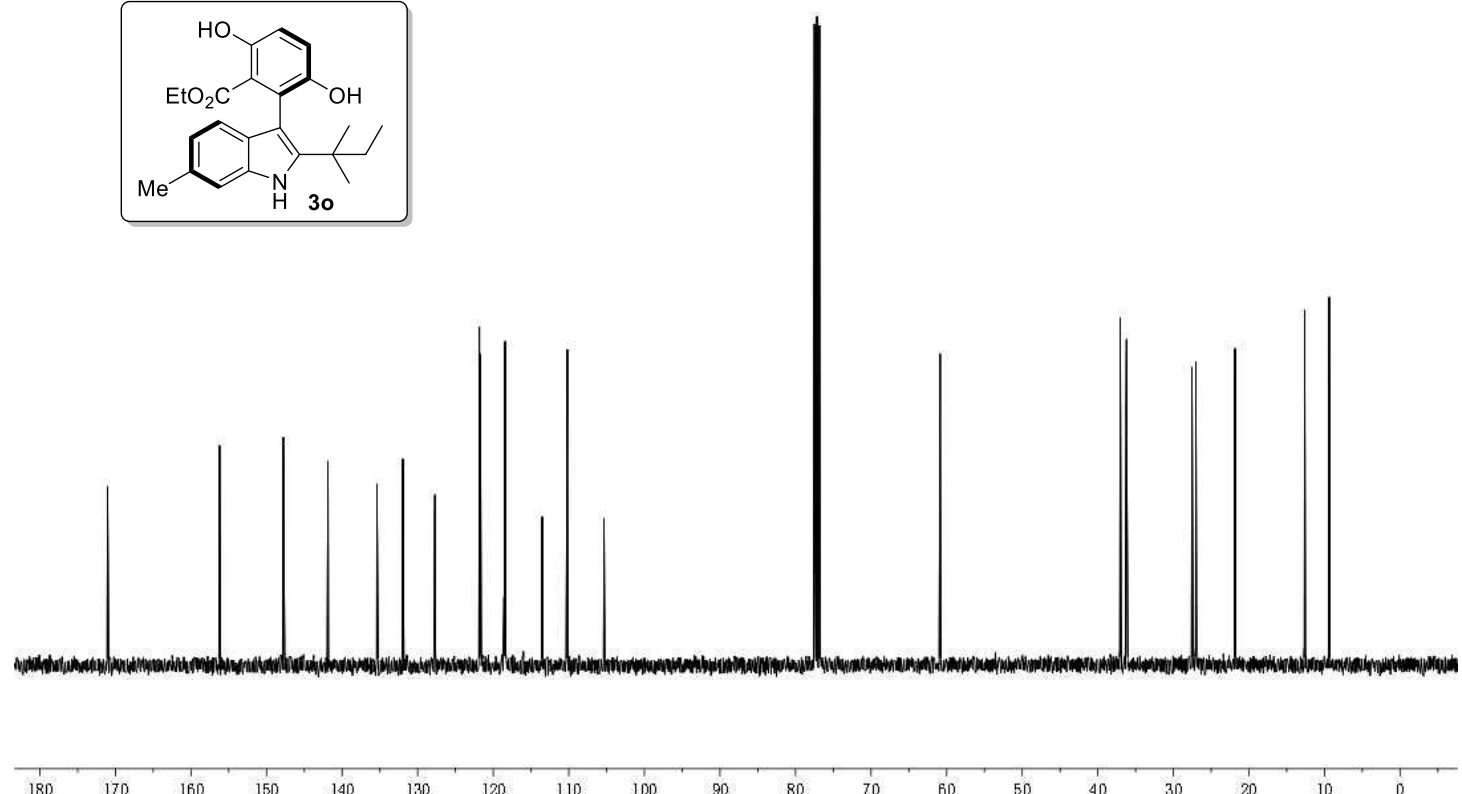

$130 \quad 120$

$\mathrm{fl}^{90}(\mathrm{pgm})$

$60 \quad 50$

$30,20,10,1$, 

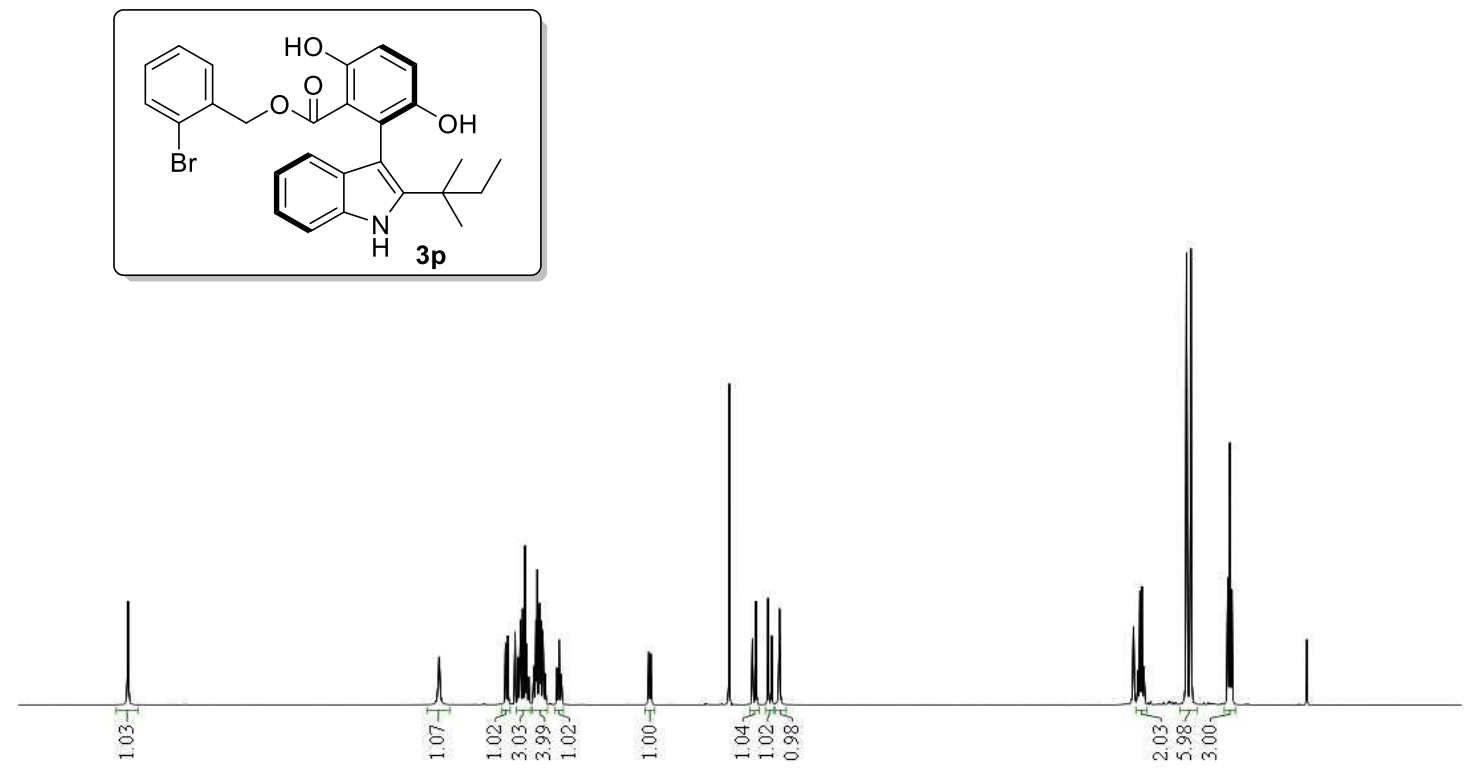

$\begin{array}{lllllllllllllllllllllllllllllllllllll}11.5 & 11.0 & 10.5 & 10.0 & 9.5 & 9.0 & 8.5 & 8.0 & 7.5 & 7.0 & 6.5 & 6.0 & 5.5 & 5.0 & 4.5 & 4.0 & 3.5 & 3.0 & 2.5 & 2.0 & 1.5 & 1.0 & 0.5 & 0.0 & -0.5 & -1.0\end{array}$

5. \%

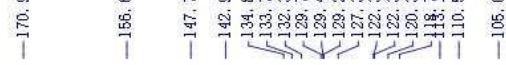

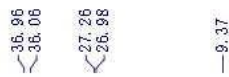

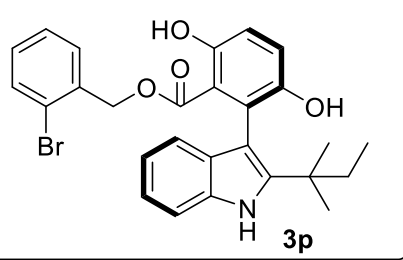

$\begin{array}{lllllllllll}90 & 180 & 170 & 160 & 150 & 140 & 130 & 120 & 110 & 100 & 90\end{array}$ 

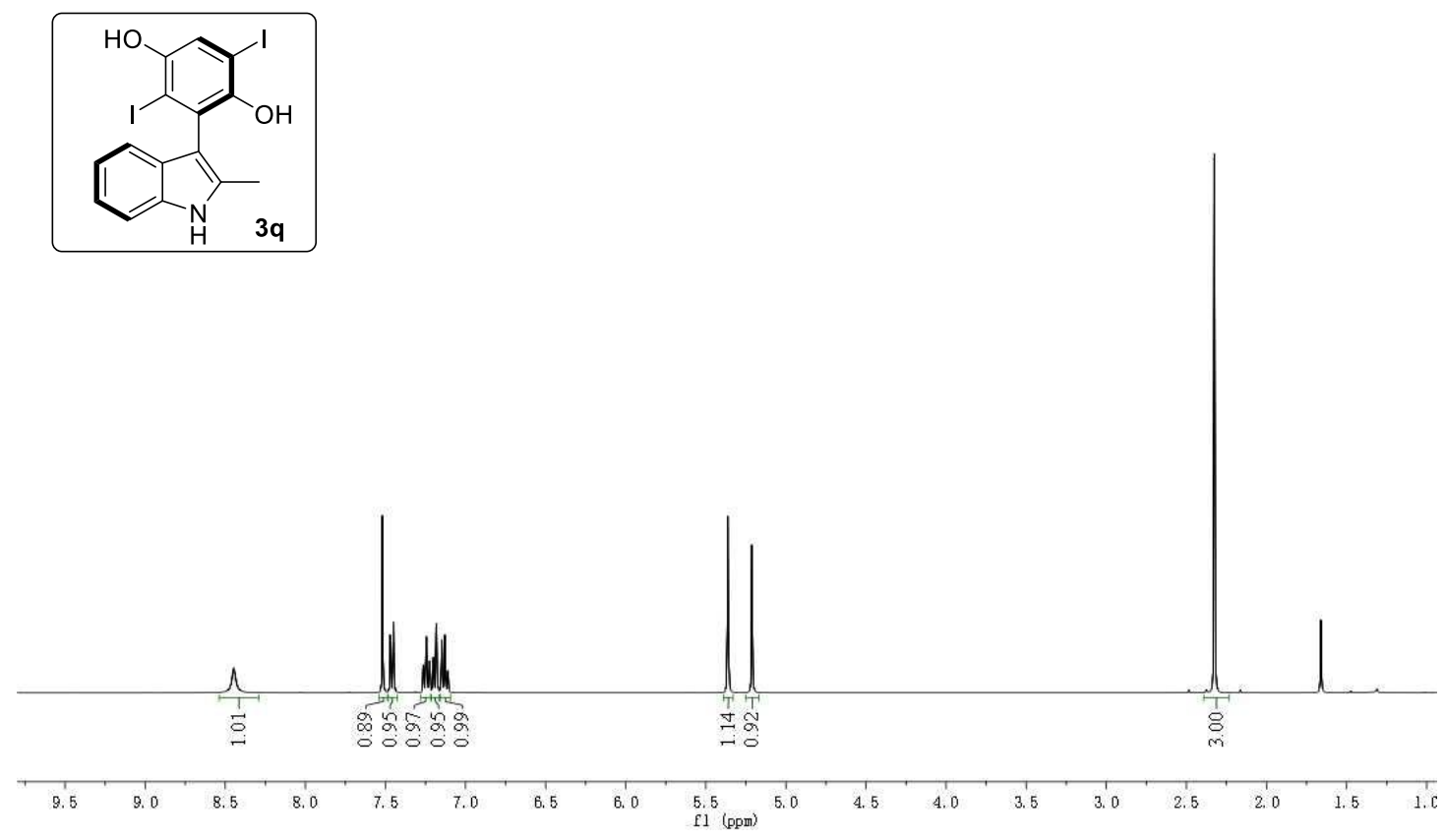

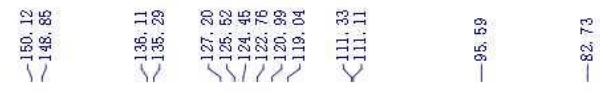

5
$\substack{1 \\ \cdots}$
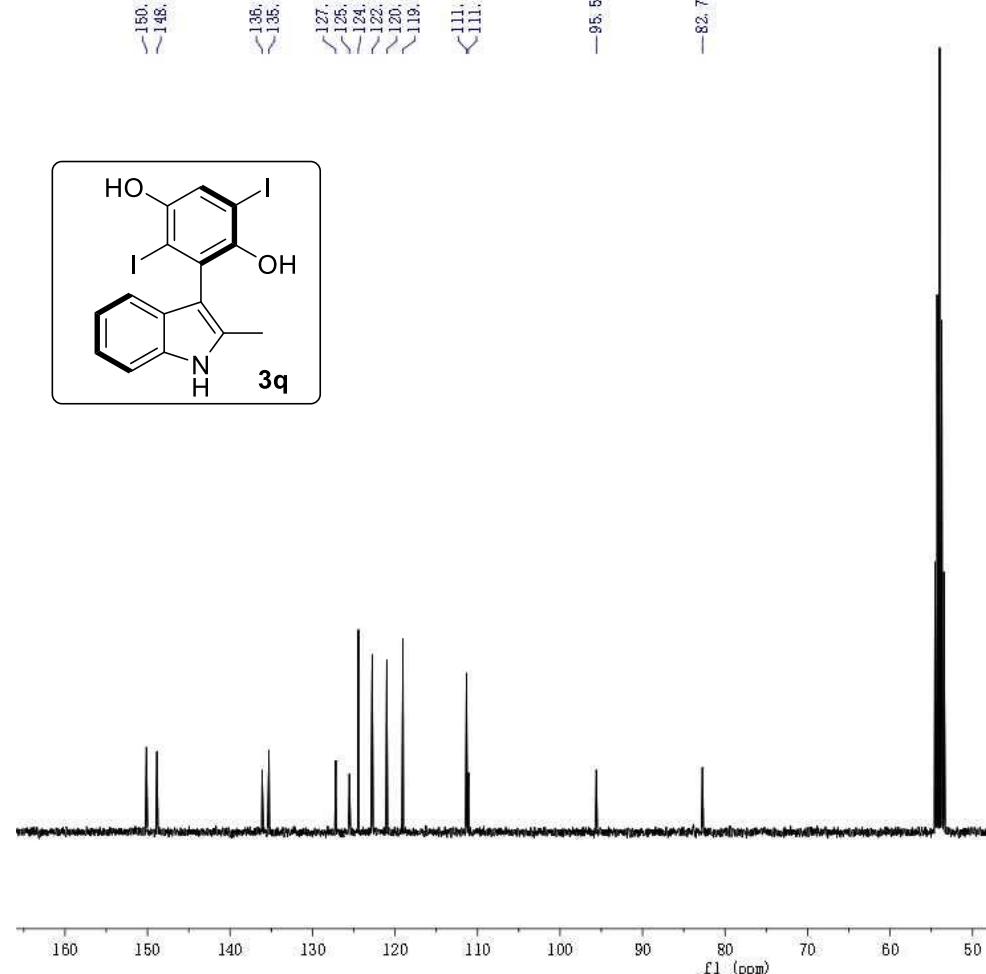

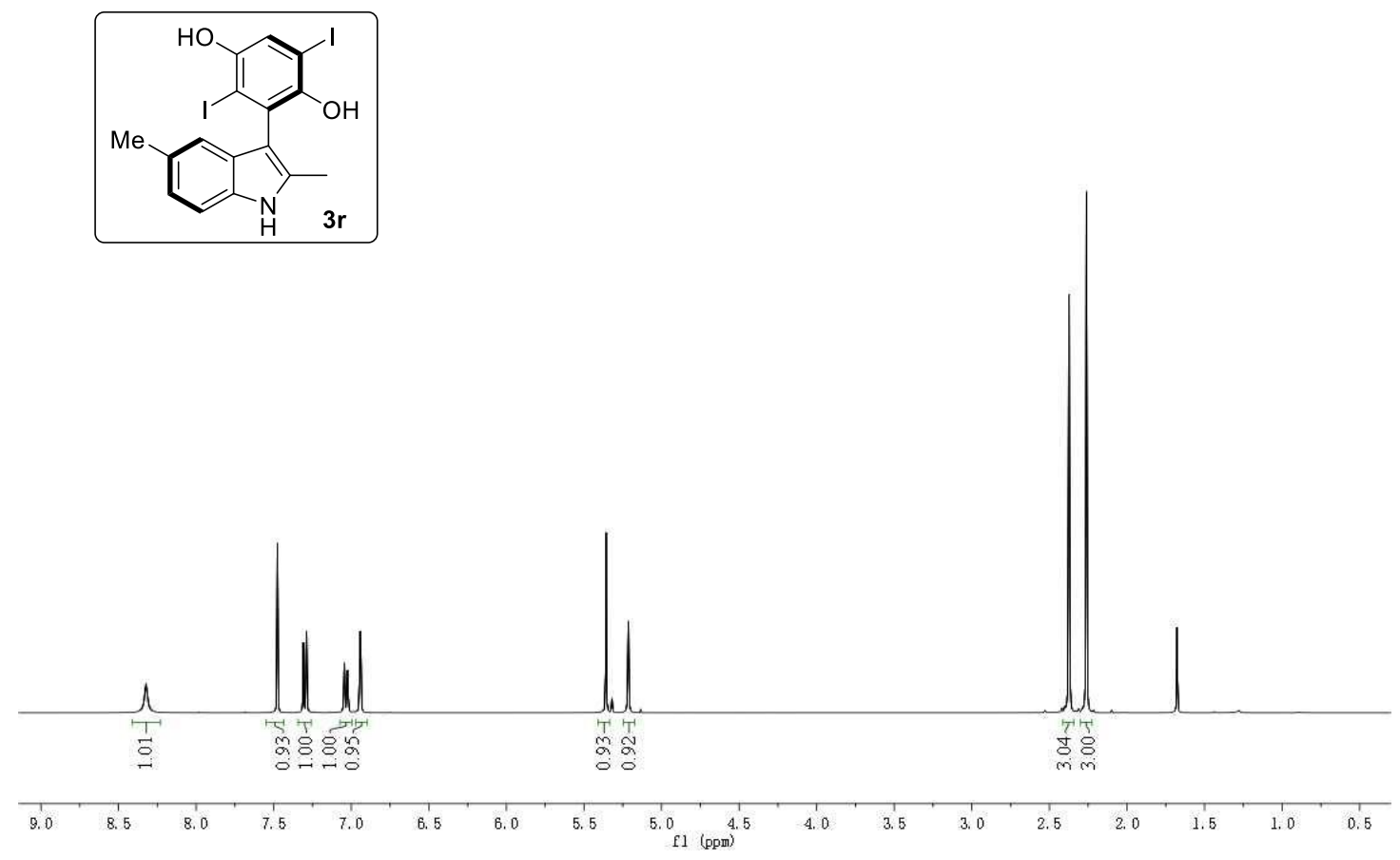

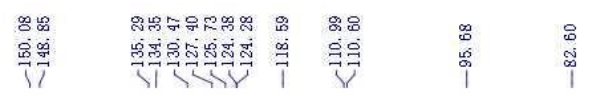
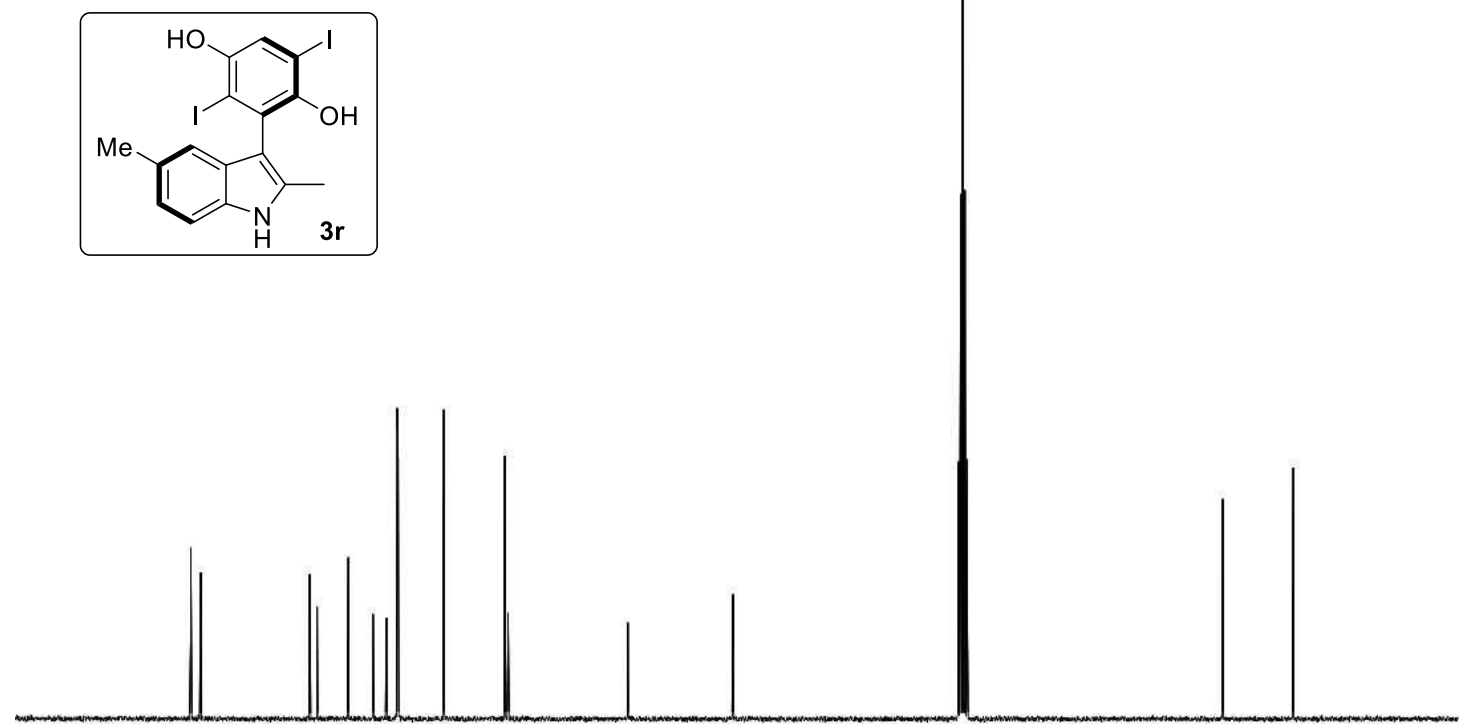

$\frac{1}{170}$
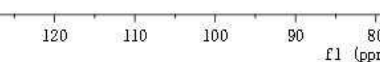

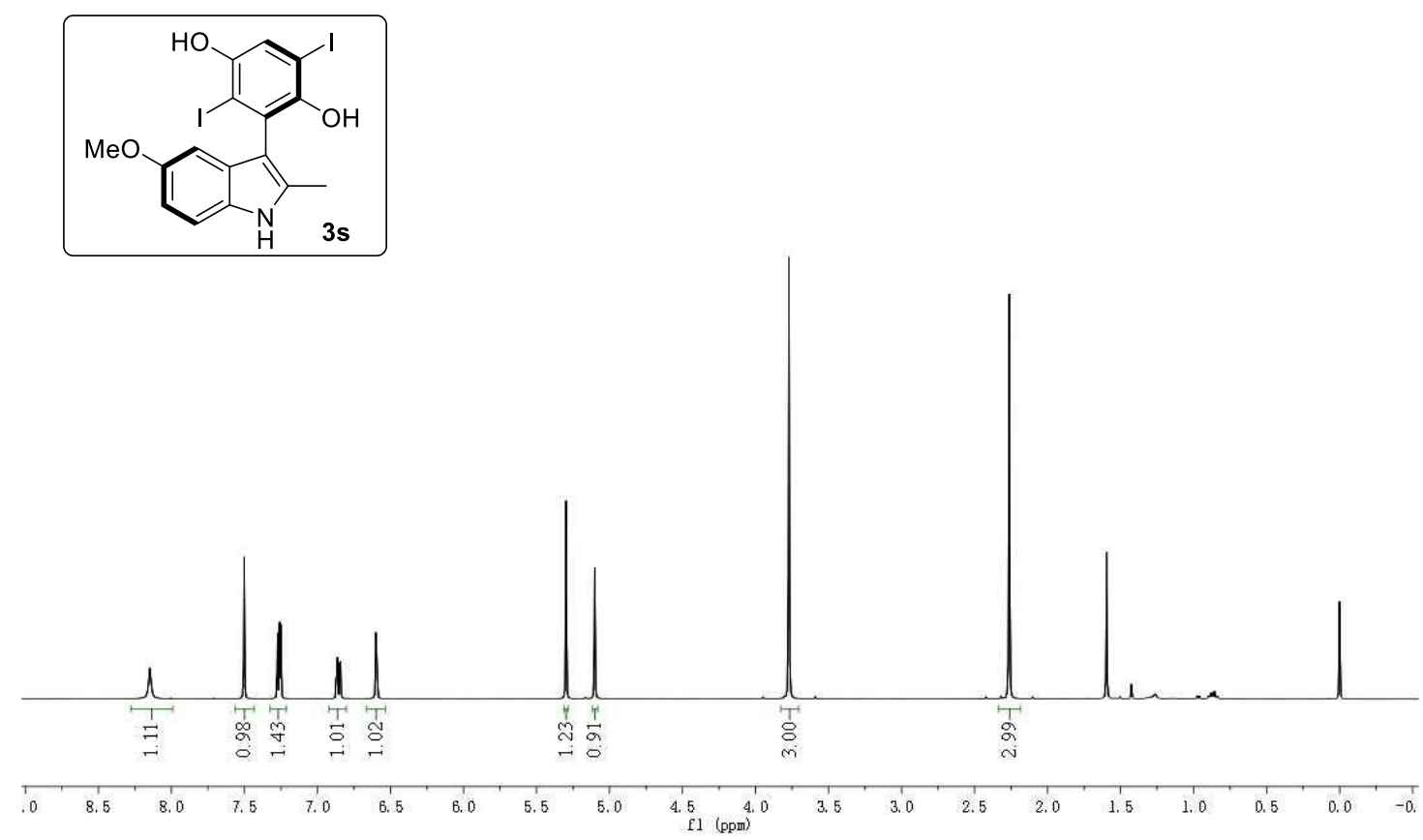

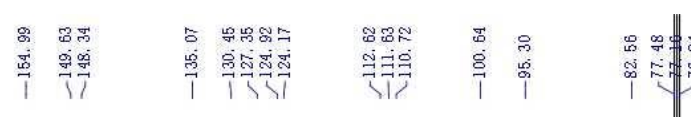
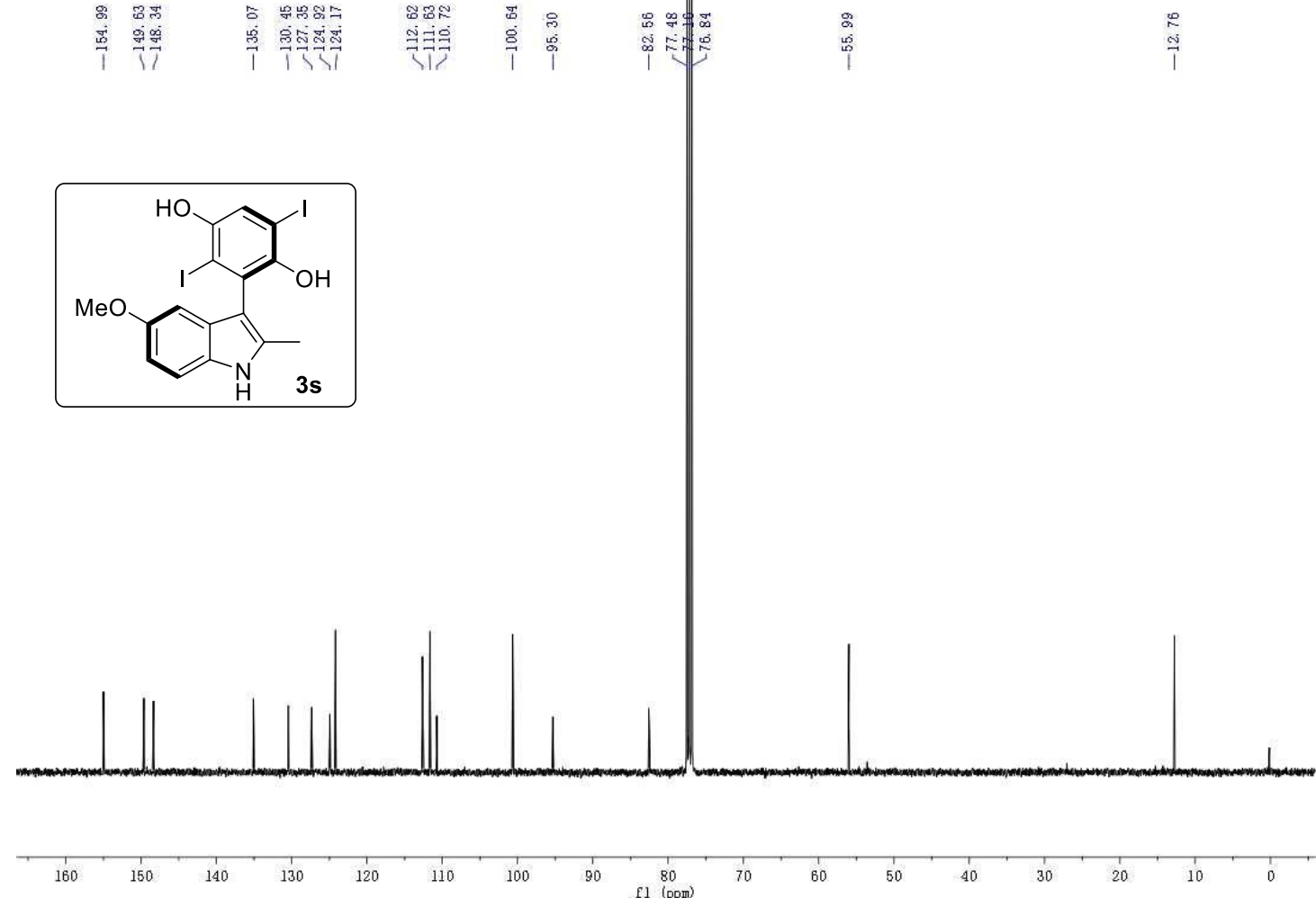

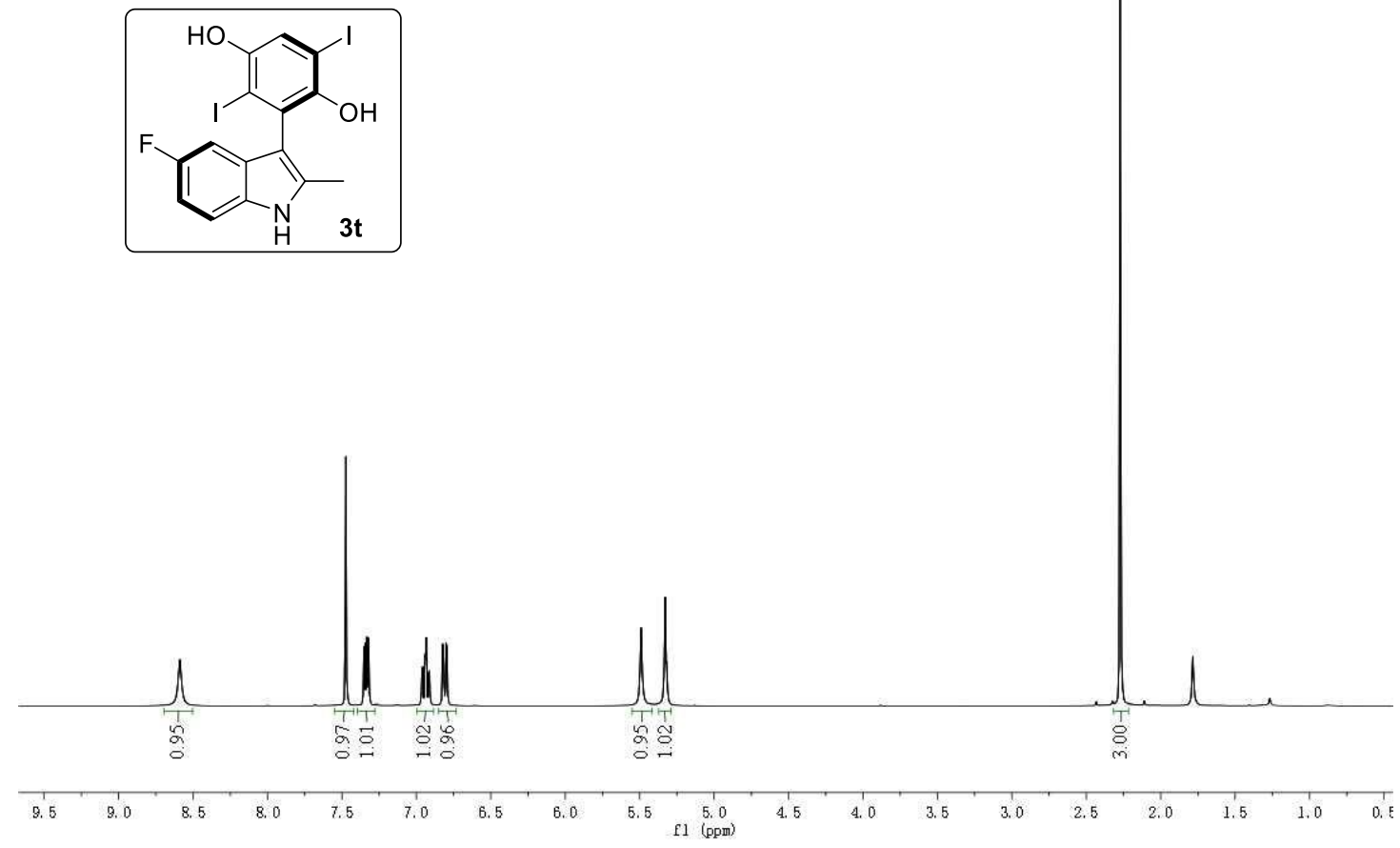

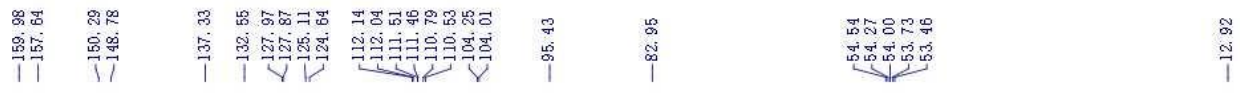
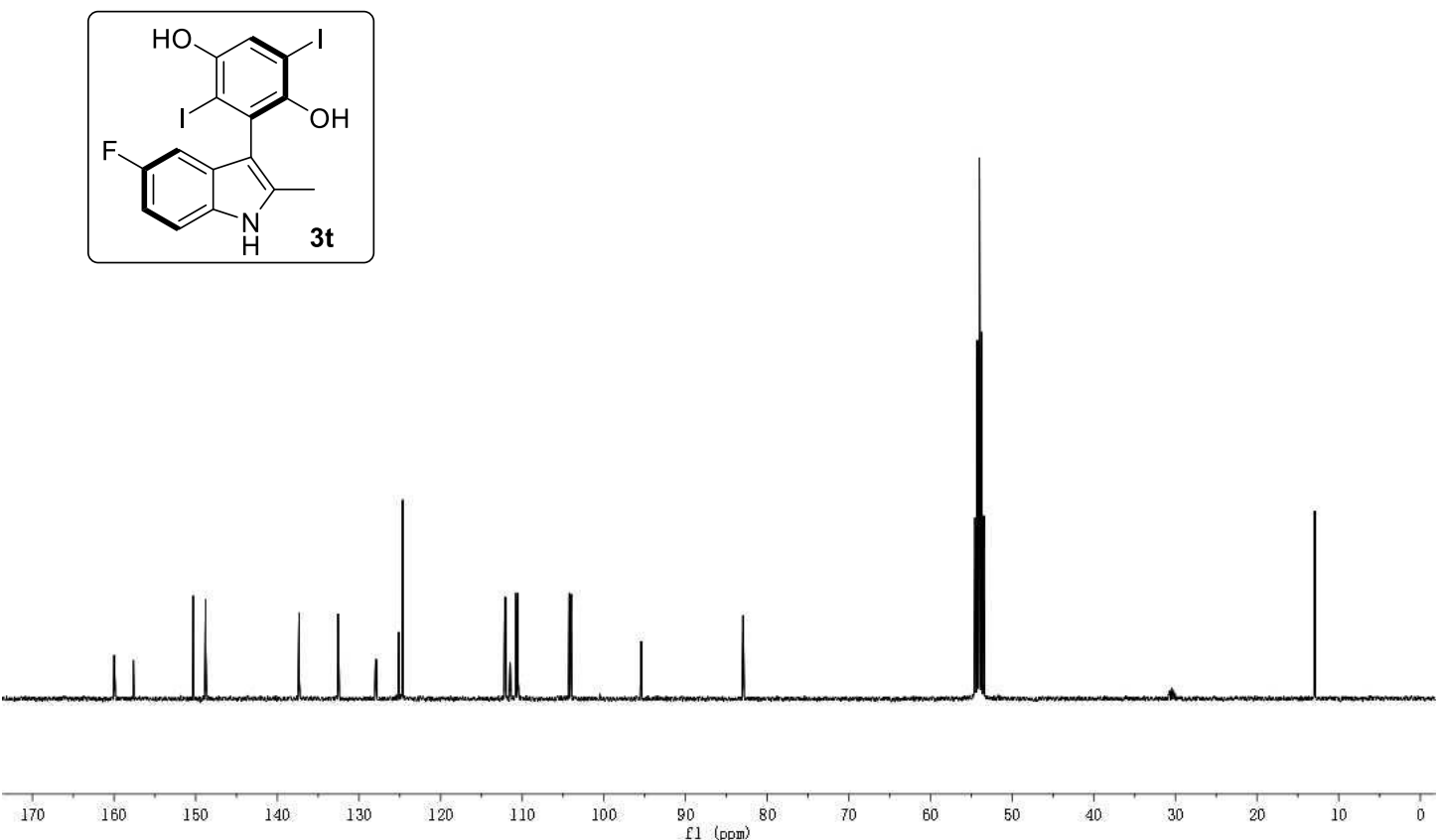

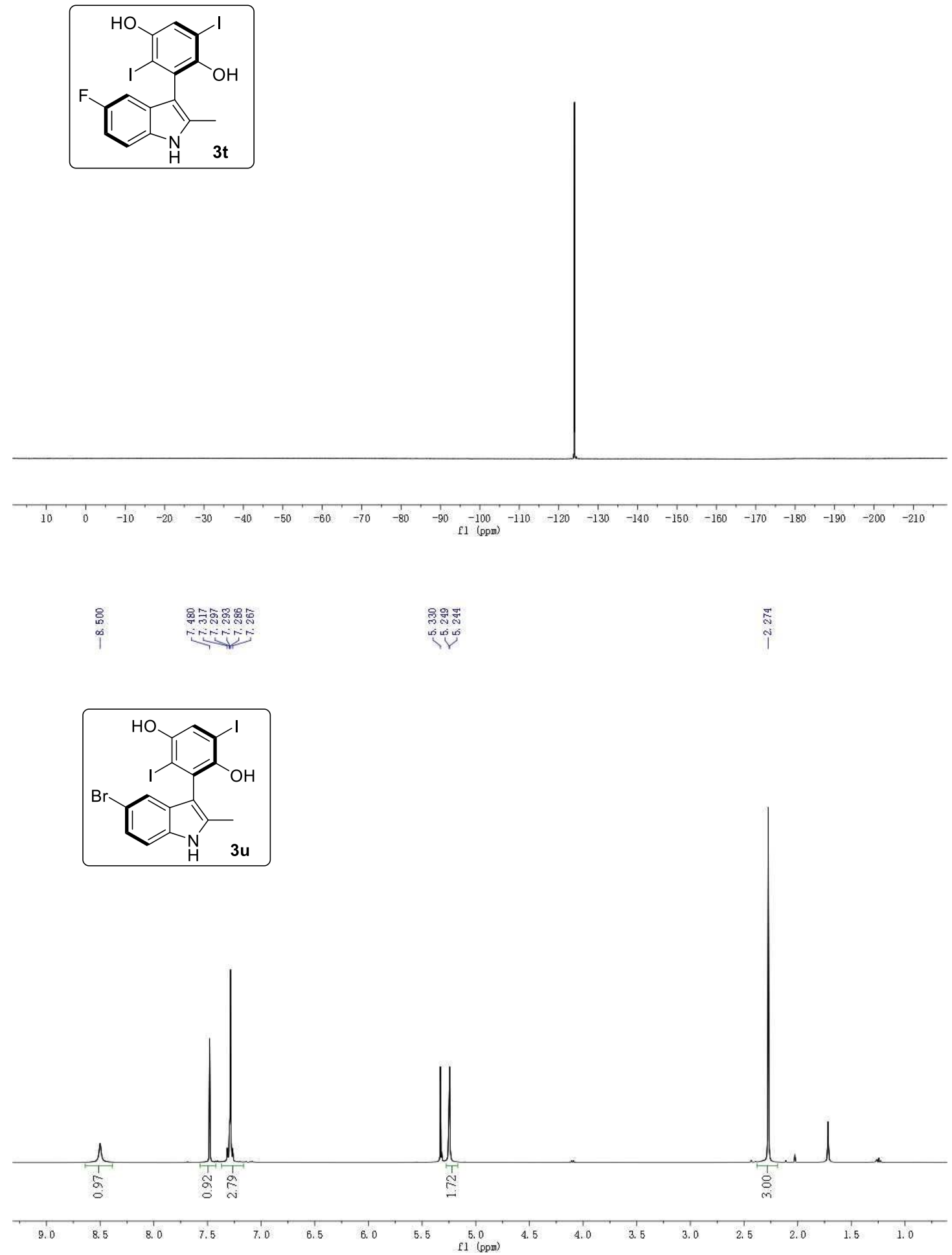
<smiles>Cc1[nH]c2ccc(Br)cc2c1-c1c(O)c(I)cc(O)c1I</smiles>
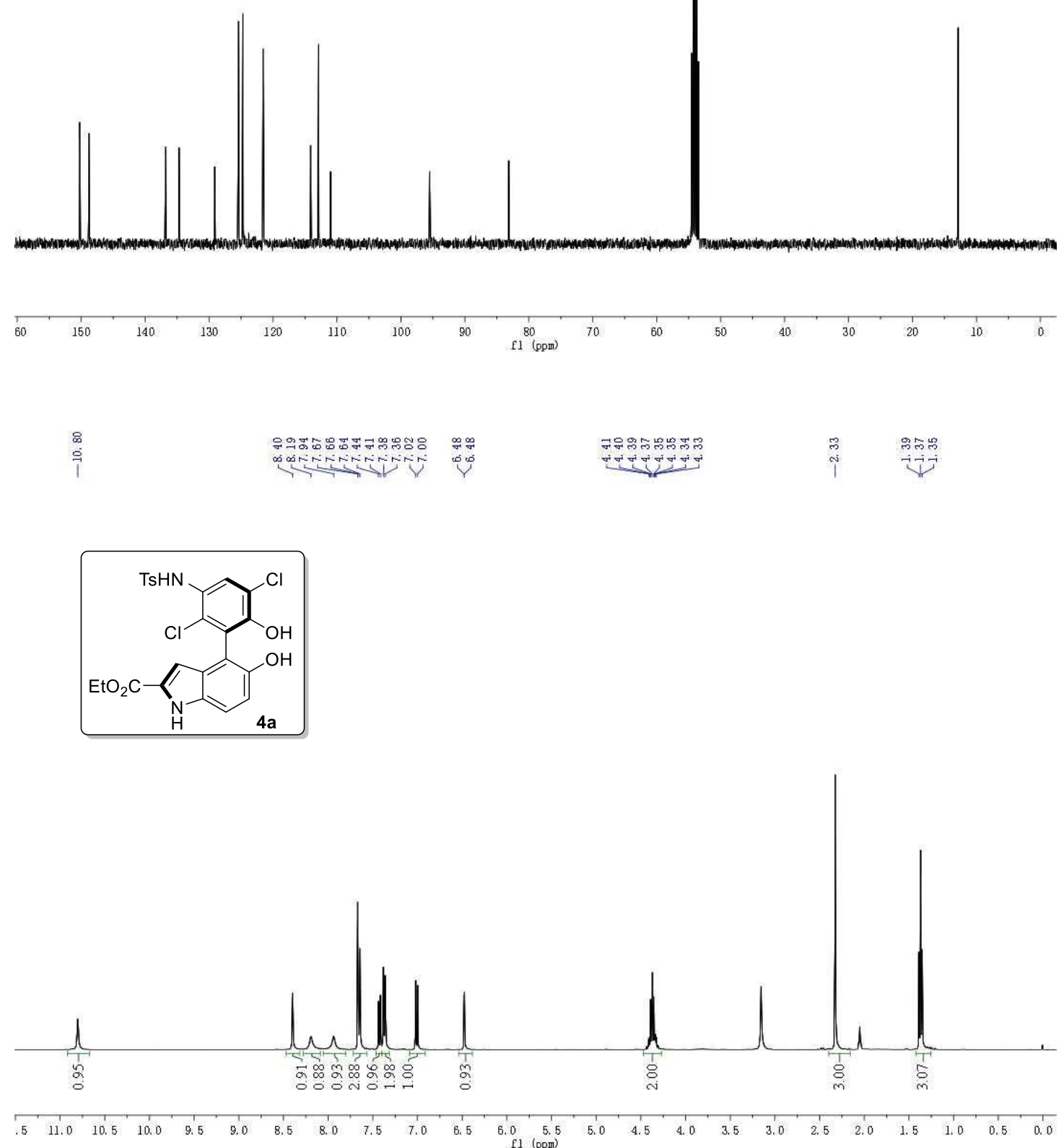

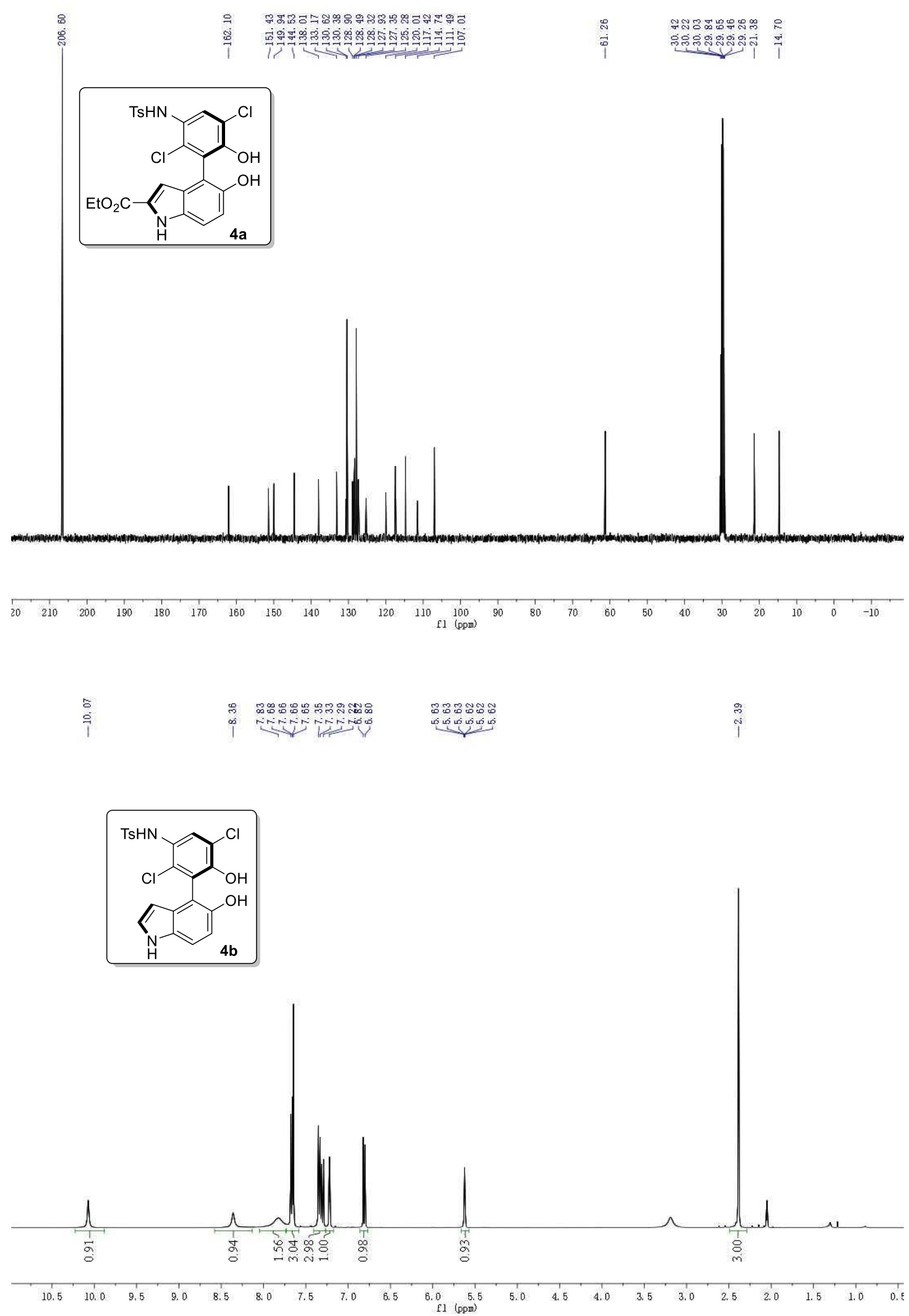

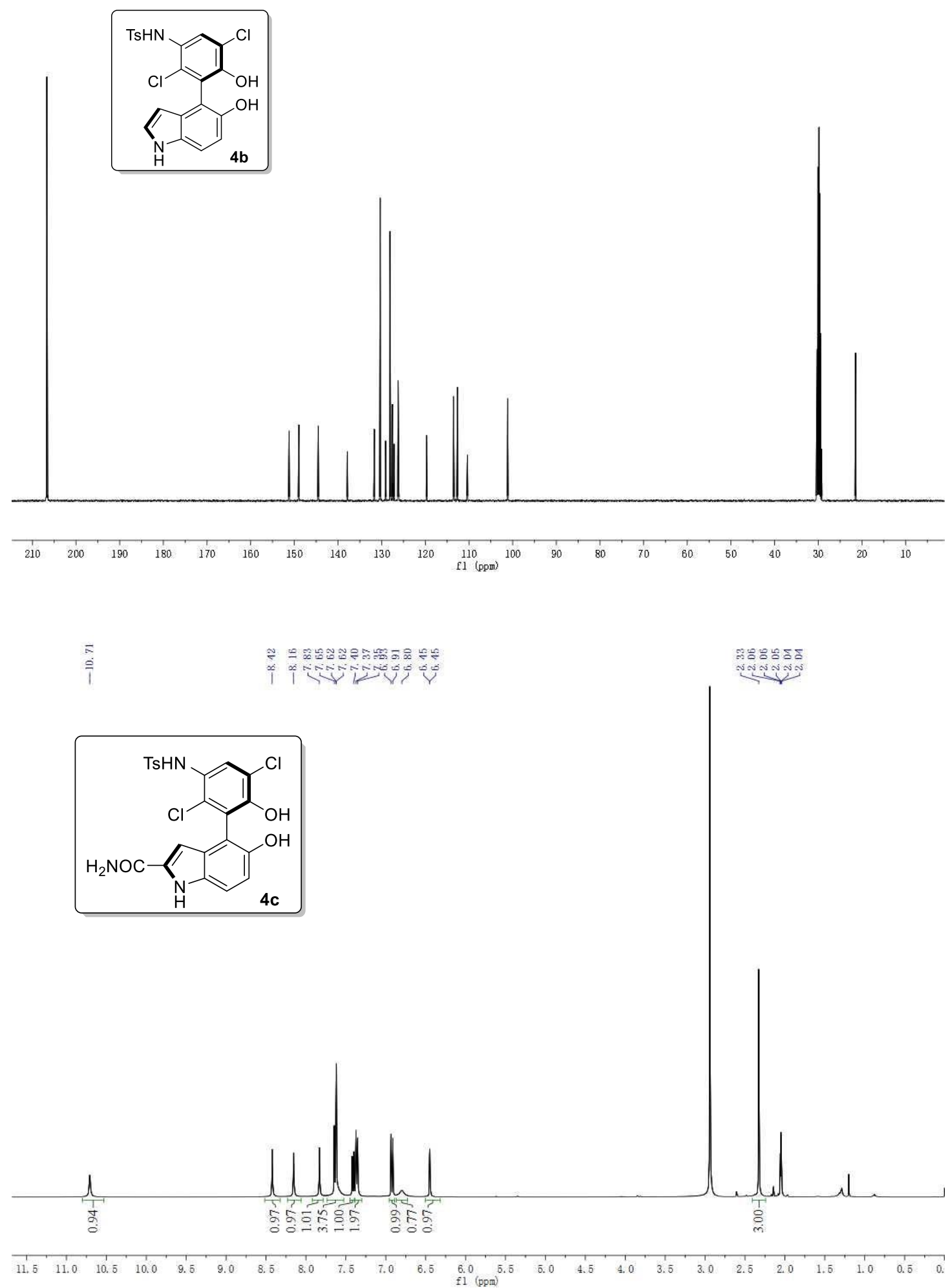

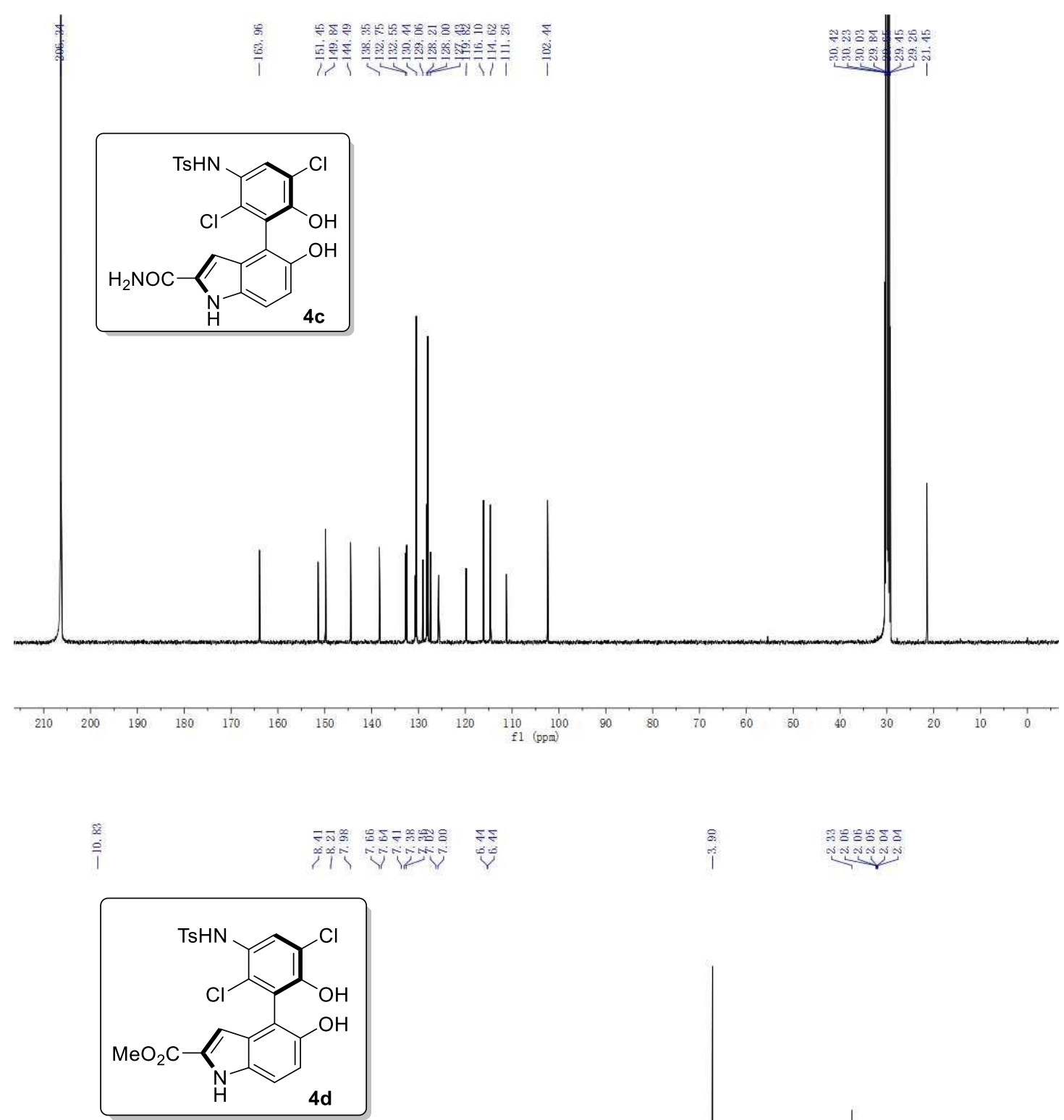

4d

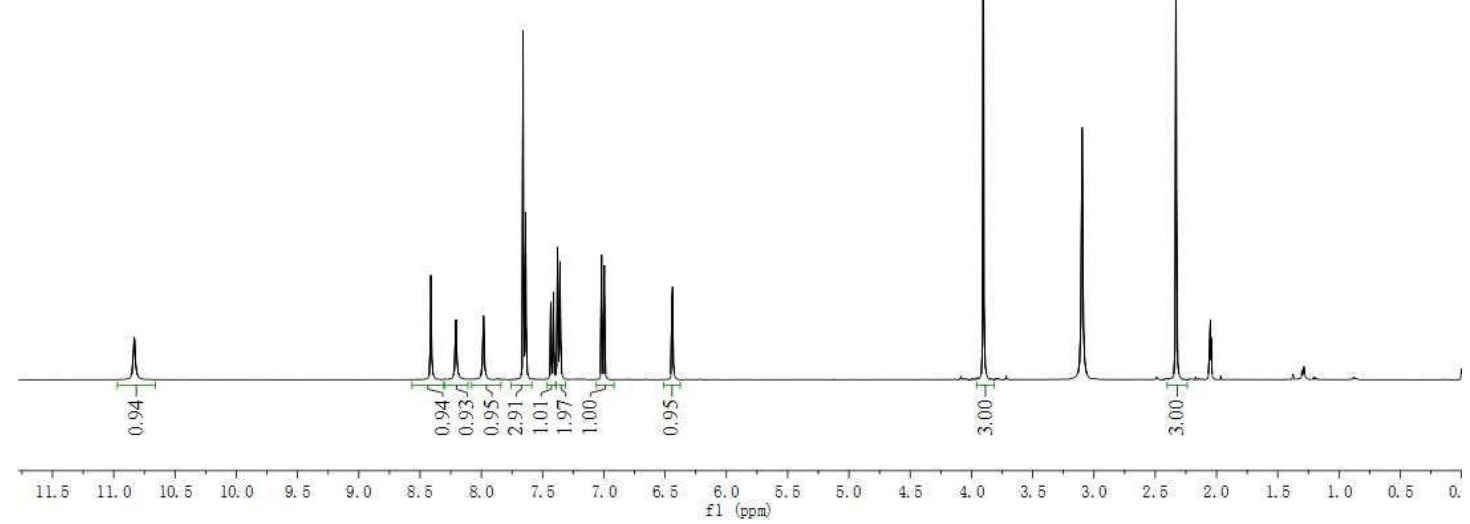



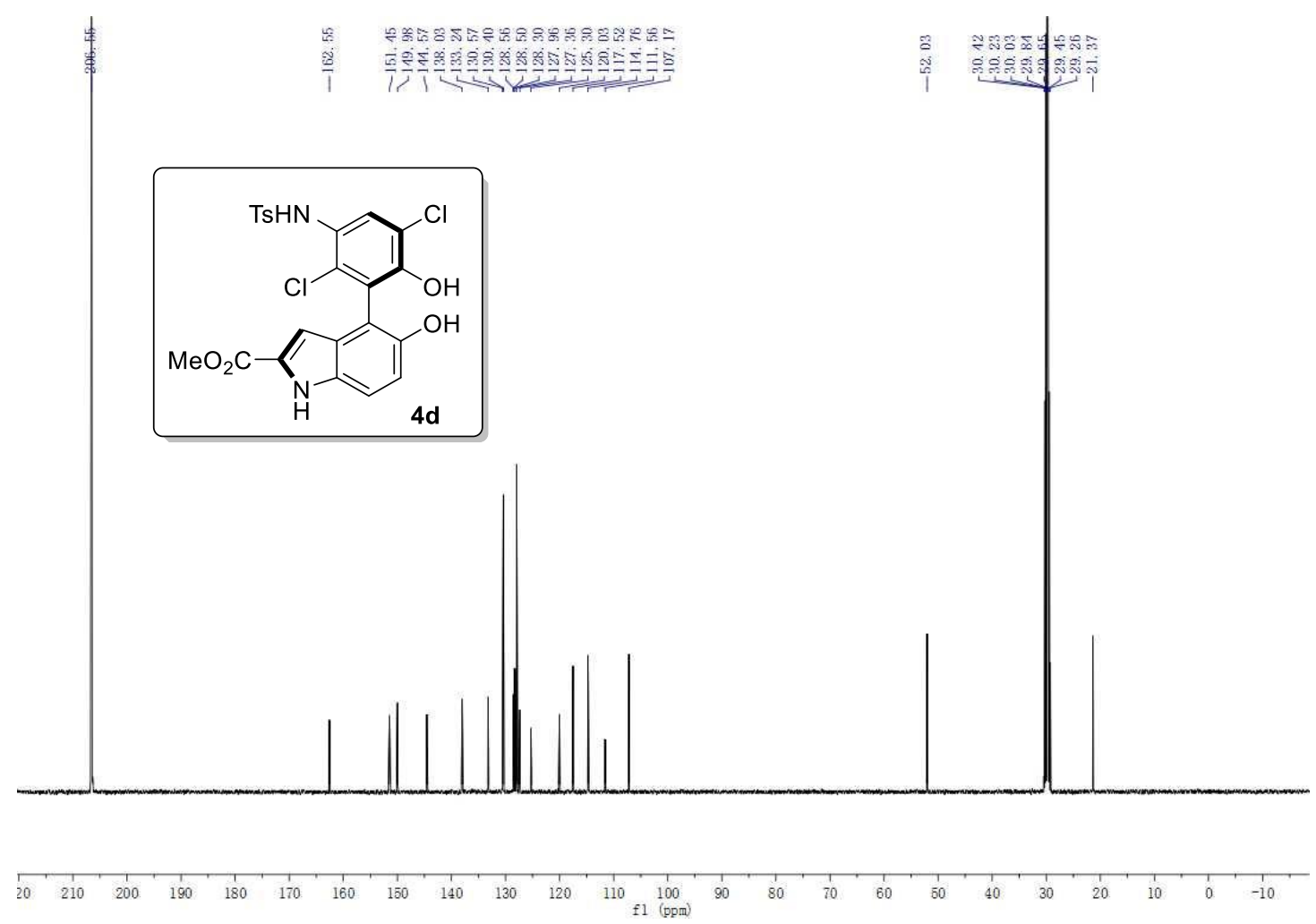

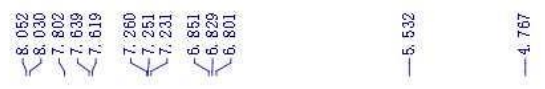

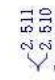
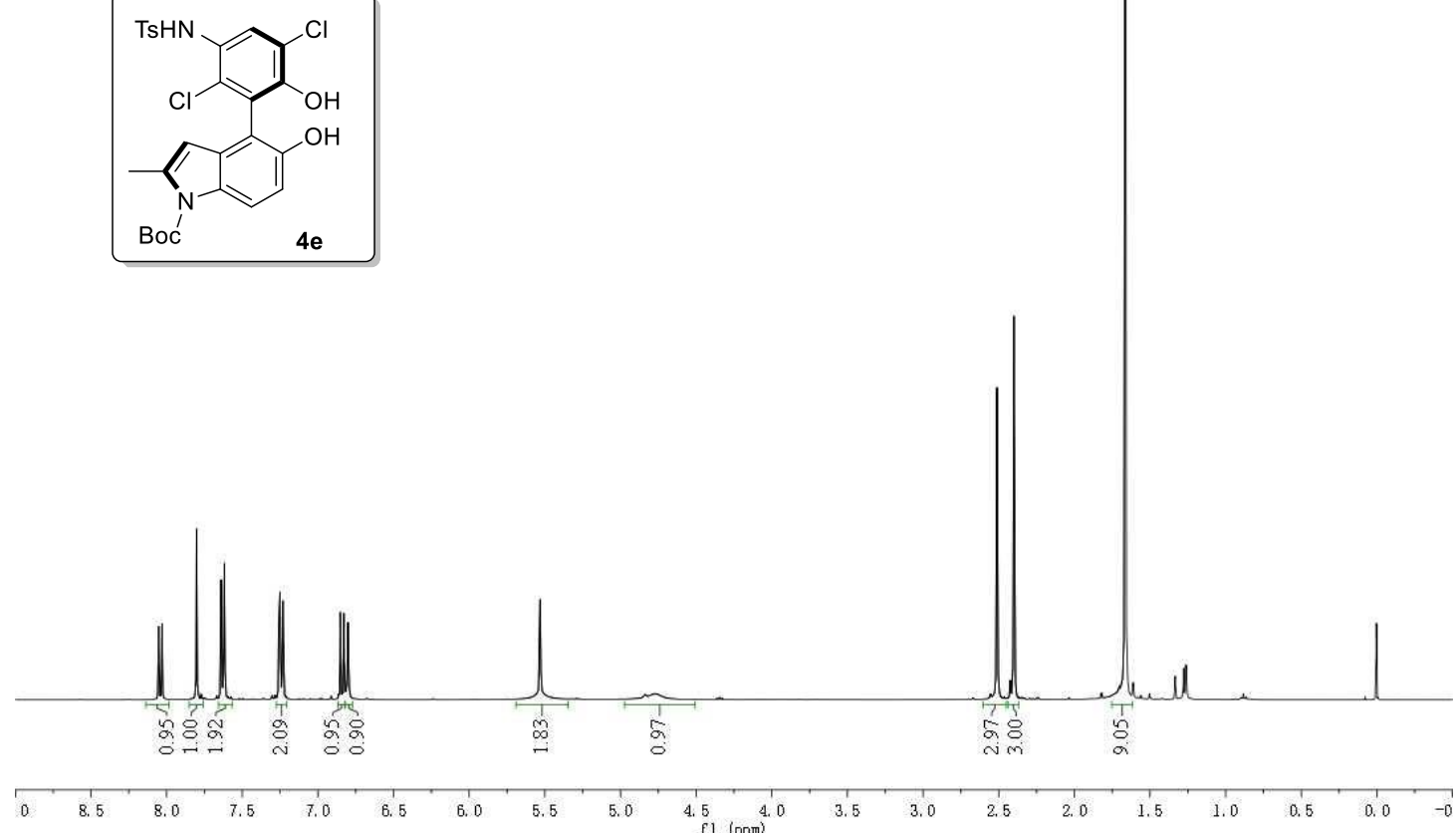


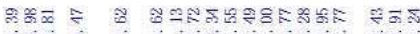

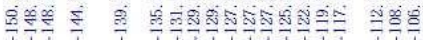
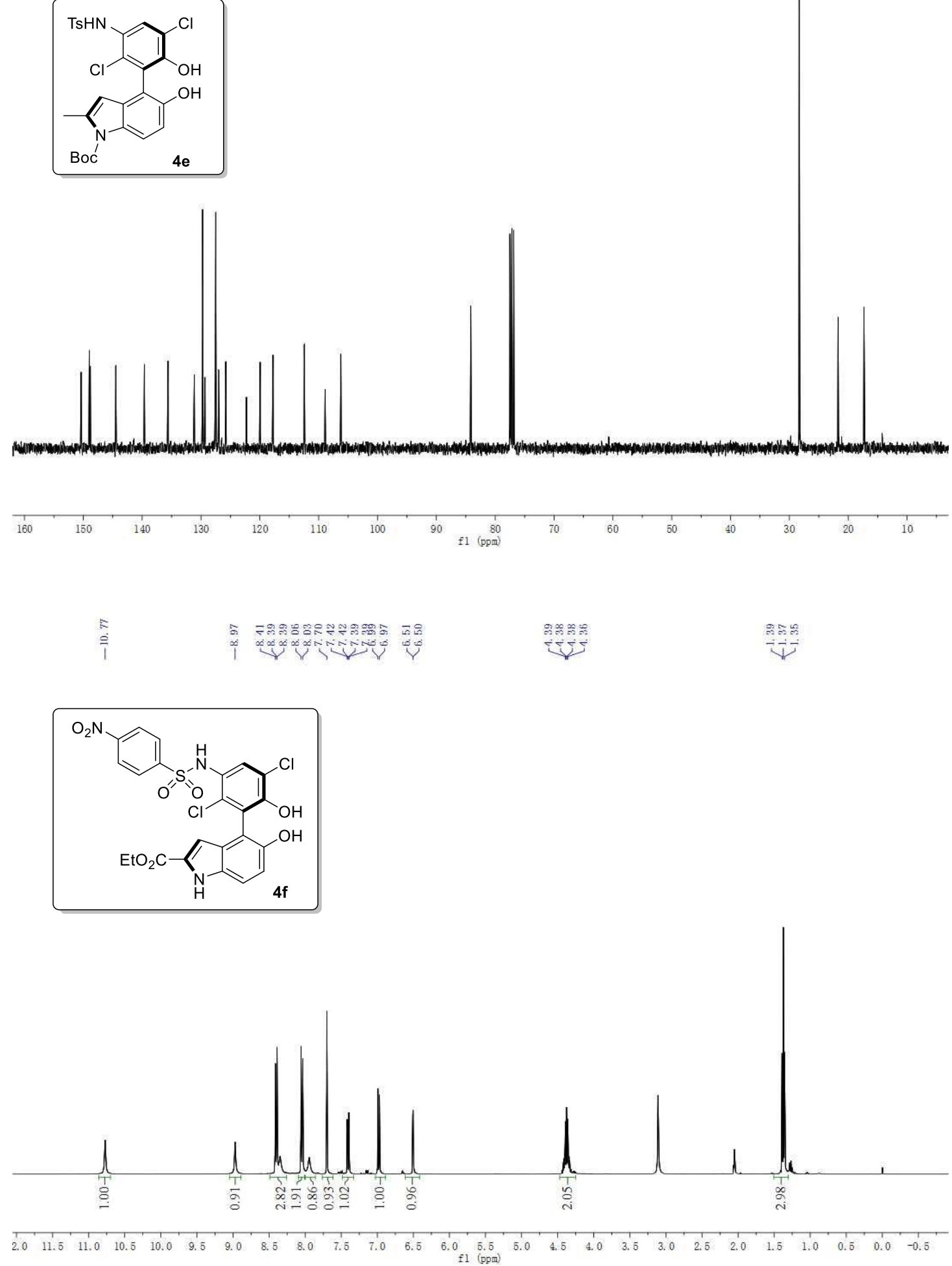

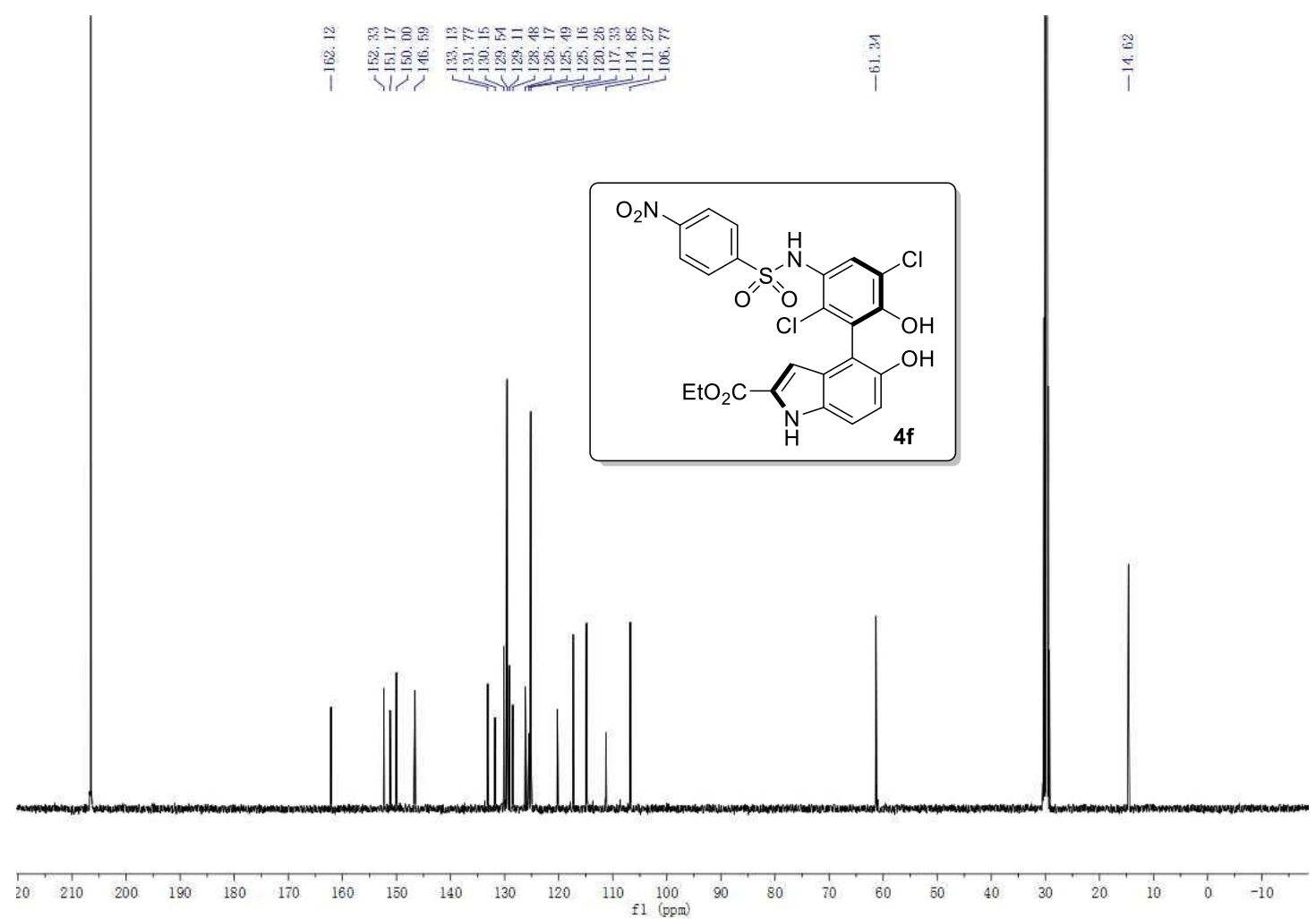

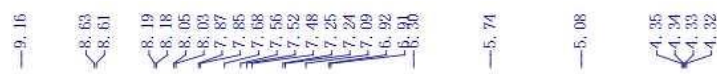

开<smiles>CCOC(=O)c1cc2c(-c3c(NS(=O)(=O)c4ccc5ccccc5c4)ccc(O)c3O)c(Cl)cc(Cl)c2[nH]1</smiles>

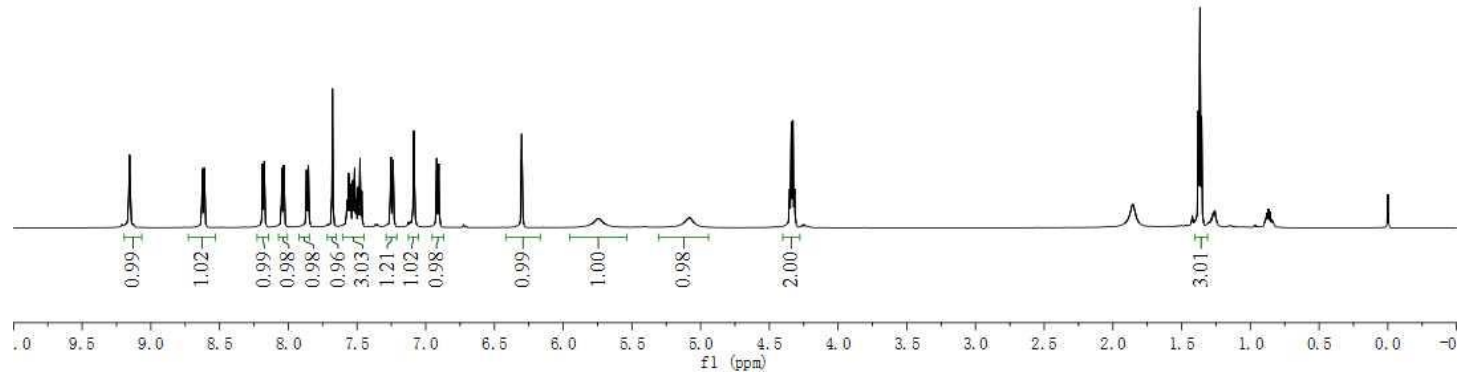




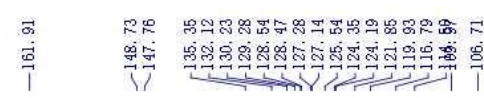

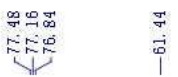

$\overrightarrow{\overrightarrow{0}}$<smiles>CCOC(=O)C1Cc2c(ccc(O)c2-c2c(O)c(Cl)cc(NS(=O)(=O)c3ccc4ccccc4c3)c2O)N1</smiles>
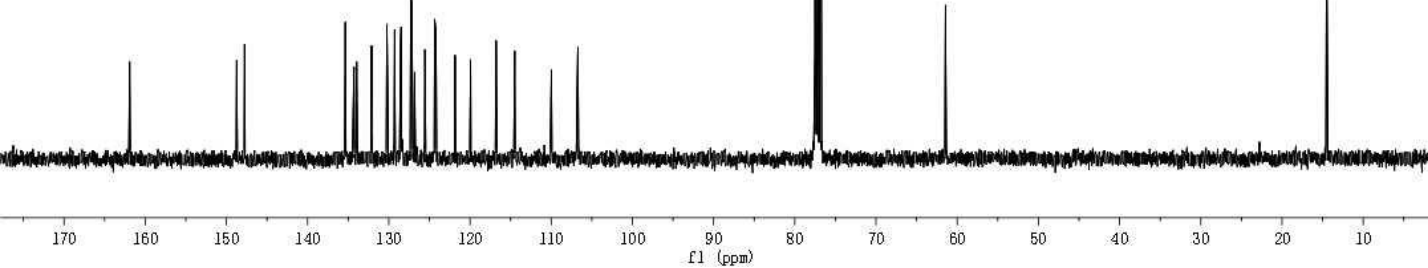

品恣品

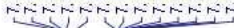

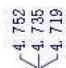

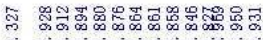

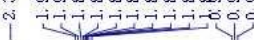
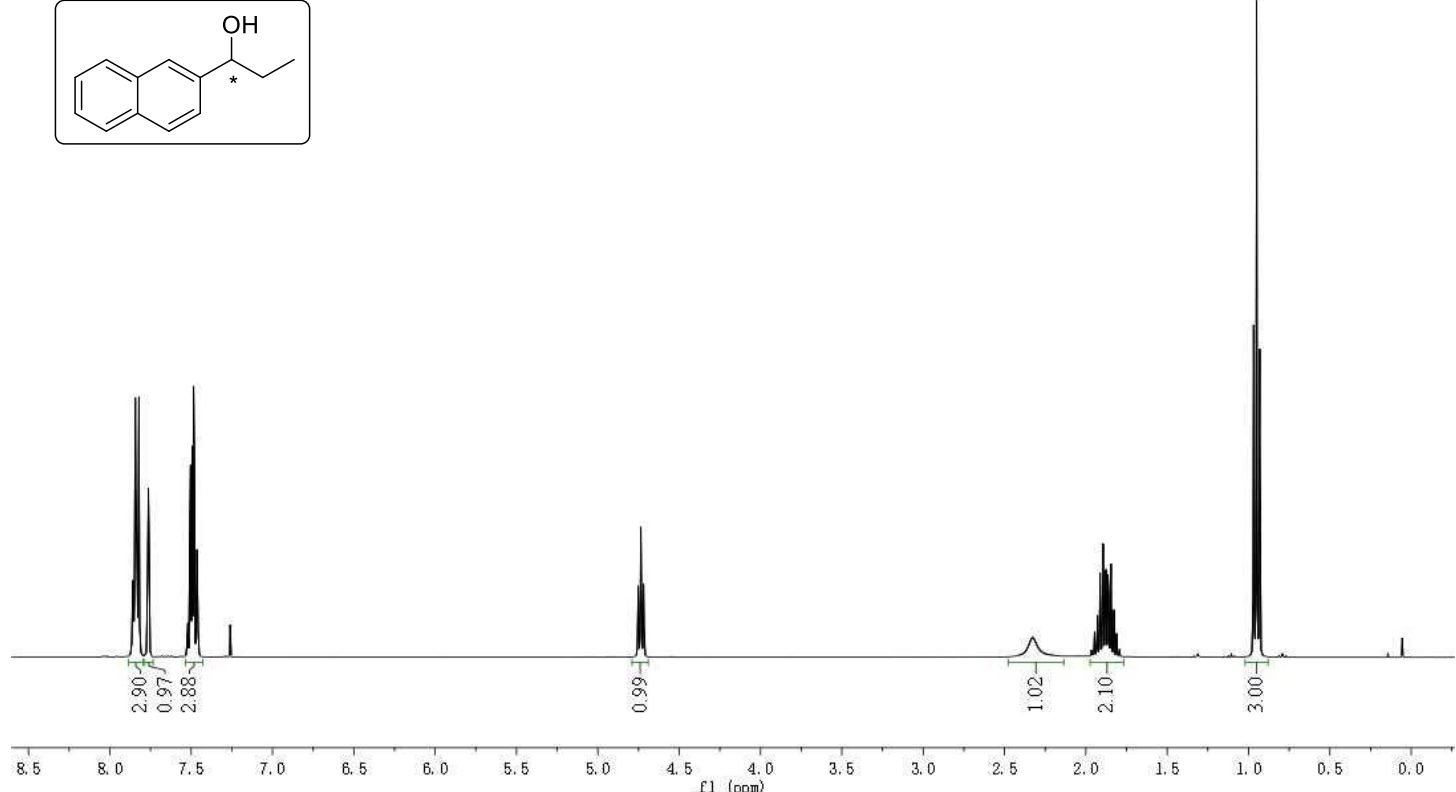

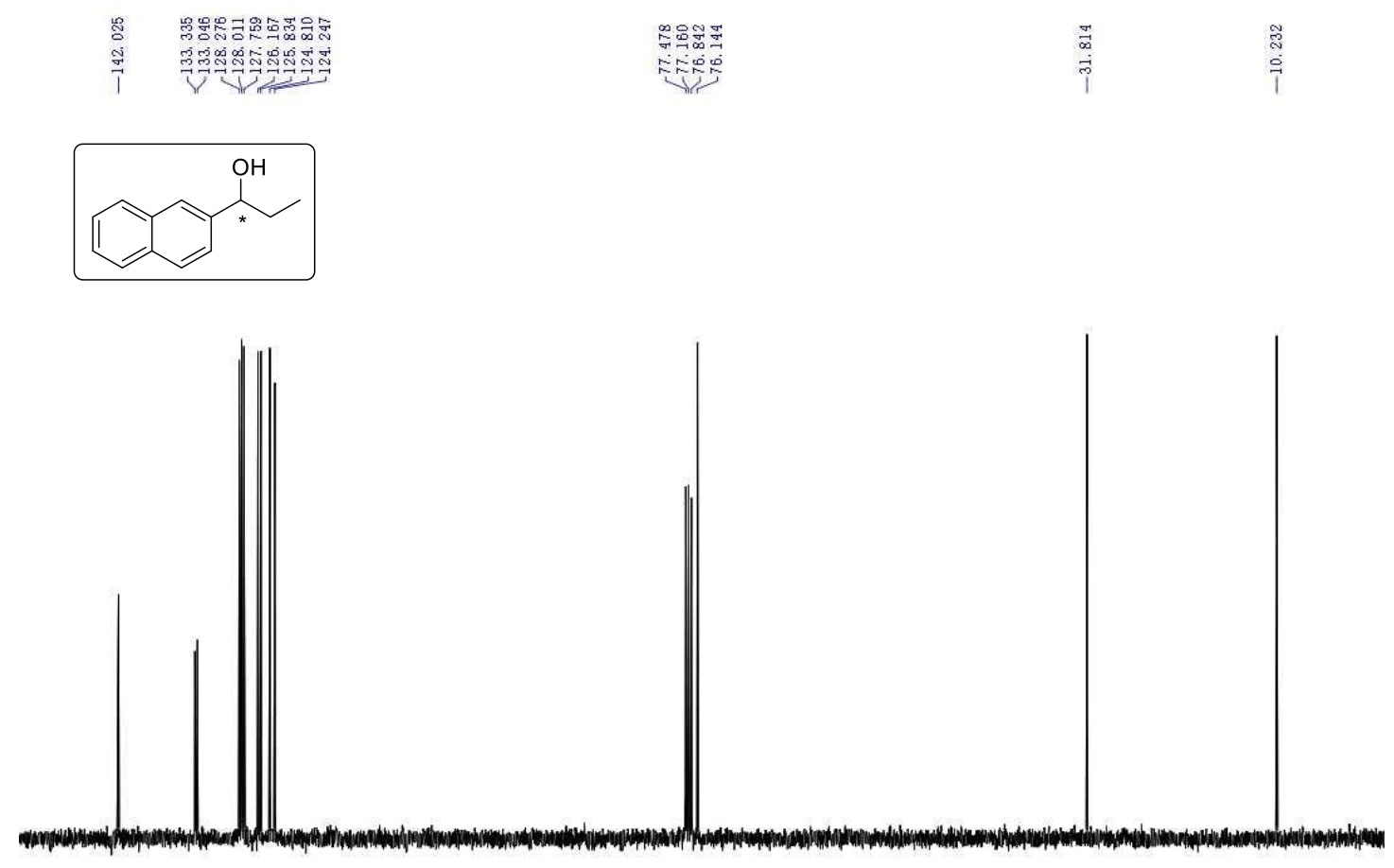

$\begin{array}{llllllllllllllllllllllllllllllllllllllllllll}150 & 145 & 140 & 135 & 130 & 125 & 120 & 115 & 110 & 105 & 100 & 95 & 90 & 85 & 80 & 75 & 70 & 65 & 60 & 55 & 50 & 45 & 40 & 35 & 30 & 25 & 20 & 15 & 10 & 5 & 0\end{array}$ 